Probability Surveys

Vol. 17 (2020) 545-676

ISSN: $1549-5787$

https://doi.org/10.1214/19-PS337

\title{
Local characteristics and tangency of vector-valued martingales
}

\author{
Ivan S. Yaroslavtsev \\ Max Planck Institute for Mathematics in the Sciences \\ Inselstraße 22 \\ 04103 Leipzig \\ Germany \\ Delft Institute of Applied Mathematics \\ Delft University of Technology \\ P.O. Box 5031 \\ 2600 GA Delft \\ The Netherlands \\ e-mail: yaroslavtsev.i.s@yandex.ru
}

\begin{abstract}
This paper is devoted to tangent martingales in Banach spaces. We provide the definition of tangency through local characteristics, basic $L^{p}$ - and $\phi$-estimates, a precise construction of a decoupled tangent martingale, new estimates for vector-valued stochastic integrals, and several other claims concerning tangent martingales and local characteristics in infinite dimensions. This work extends various real-valued and vector-valued results in this direction e.g. due to Grigelionis, Hitczenko, Jacod, Kallenberg, Kwapien, McConnell, and Woyczyński. The vast majority of the assertions presented in the paper is done under the necessary and sufficient UMD assumption on the corresponding Banach space.
\end{abstract}

MSC2020 subject classifications: Primary 60G44, 60B11; secondary 60G51, 60G57, 60H05, 46G12, 28A50.

Keywords and phrases: Tangent martingales, decoupling, local characteristics, UMD Banach spaces, canonical decomposition, stochastic integration, Lévy-Khinchin formula, independent increments.

Received August 2019.

\section{Contents}

1 Introduction . . . . . . . . . . . . . . . . . . 546

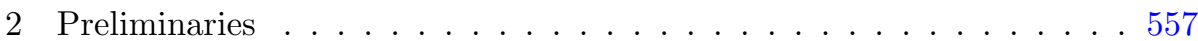

2.1 Enlargement of a filtered probability space . . . . . . . . . 557

2.2 Conditional expectation on a product space. Conditional proba-

bility and conditional independence . . . . . . . . . . . 558

2.3 The UMD property . . . . . . . . . . . . . . . . . . 560

2.4 Stopping times . . . . . . . . . . . . . . . 560

2.5 Martingales: real- and Banach space-valued . . . . . . . . . . . 561

2.6 Quadratic variation . . . . . . . . . . . . . . . . 562

2.7 The canonical decomposition . . . . . . . . . . . . . . 563

2.8 Random measures . . . . . . . . . . . . . . . . . . . . . . 564 
2.9 Poisson random measures . . . . . . . . . . . . . . . 566

2.10 Stochastic integration . . . . . . . . . . . . . 567

$2.11 \gamma$-radonifying operators . . . . . . . . . . . . . 568

2.12 Tangent martingales: the discrete case . . . . . . . . . . . . 569

3 Tangent martingales: the continuous-time case . . . . . . . . . . . . 570

3.1 Local characteristics and tangency . . . . . . . . . . . . . 570

3.2 Local characteristics and canonical decomposition . . . . . . . . 573

3.3 Continuous martingales . . . . . . . . . . . . . . . 575

3.4 Stochastic integrals with respect to random measures . . . . . . 578

3.4 .1 Cox process . . . . . . . . . . . . . . 579

3.4.2 Random measures: tangency and decoupling . . . . . . 579

3.5 Purely discontinuous quasi-left continuous martingales . . . . . . 593

3.6 Purely discontinuous martingales with accessible jumps . . . . 599

3.7 Proof of Theorem 3.7 and $3.8 \ldots \ldots$. . . . . . . . . 605

3.8 Uniqueness of a decoupled tangent martingale . . . . . . . . . 606

3.9 Independent increments given the local characteristics . . . . . 607

4 Upper bounds and the decoupling property . . . . . . . . . . . 607

5 Convex functions with moderate growth . . . . . . . . . . . . . 611

5.1 Good- $\lambda$ inequalities . . . . . . . . . . . . . . . . . . 611

5.2 Proof of Theorem 5.1 . . . . . . . . . . . . . . . . 614

5.3 Not convex functions . . . . . . . . . . . . . . . 618

6 Integration with respect to a general martingale . . . . . . . . . . 620

7 Weak tangency versus tangency . . . . . . . . . . . . . . . . 622

8 Decoupled tangent martingales and the recoupling property . . . . . . 624

9 Independent increments . . . . . . . . . . . . . . . . . 632

9.1 Weak local characteristics and independent increments . . . . . 632

9.2 General form of a martingale with independent increments . . . . 633

10 The approach of Jacod, Kwapień, and Woyczyński . . . . . . . . . . 642

11 Exponential formula . . . . . . . . . . . . . . . . . 653

12 Characteristic subordination and characteristic domination of martin-

gales . . . . . . . . . . . . . . . . . 658

12.1 Characteristic subordination . . . . . . . . . . . . 658

12.2 Characteristic domination . . . . . . . . . . . . . . 661

A Tangency under linear operators . . . . . . . . . . . . . . . 664

B Martingale approximations . . . . . . . . . . . . . 6667

B.1 Purely discontinuous martingales with accessible jumps . . . 667

B.2 Purely discontinuous quasi-left continuous martingales . . . . 668

Acknowledgment . . . . . . . . . . . . . . . . . . 668

References . . . . . . . . . . . . . . . . . . . . . 669

\section{Introduction}

This paper is devoted to tangent martingales. Let us start with the discrete setting. Which martingale difference sequences do we call tangent? For a Banach 
space $X$ two $X$-valued martingale difference sequences $\left(d_{n}\right)_{n \geq 1}$ and $\left(e_{n}\right)_{n \geq 1}$ are tangent if for every $n \geq 1$ a.s. ${ }^{1}$

$$
\mathbb{P}\left(d_{n} \mid \mathcal{F}_{n-1}\right)=\mathbb{P}\left(e_{n} \mid \mathcal{F}_{n-1}\right),
$$

where $\mathbb{P}\left(d_{n} \mid \mathcal{F}_{n-1}\right)(A):=\mathbb{E}\left(\mathbf{1}_{A}\left(d_{n}\right) \mid \mathcal{F}_{n-1}\right)$ and $\mathbb{P}\left(e_{n} \mid \mathcal{F}_{n-1}\right)(A):=\mathbb{E}\left(\mathbf{1}_{A}\left(e_{n}\right) \mid \mathcal{F}_{n-1}\right)$ for any Borel set $A \subset X$. This notion was first introduced by Zinn in [122] where he proved that if $X=\mathbb{R}$, then for any $p \geq 2$ the $L^{p}$ moments of $\sum_{n} d_{n}$ and $\sum_{n} e_{n}$ are comparable given $\left(d_{n}\right)$ and $\left(e_{n}\right)$ are conditionally symmetric ${ }^{2}$ (the general case $1 \leq p<\infty$ was obtained by Hitczenko in [45]). The estimates of Zinn and Hitczenko have been extended by McConnell in [73] and Hitczenko in [44] to infinite dimensions. It turned out that such estimates characterize a certain condition concerning the geometry of a Banach space, namely, the $U M D$ property (see Subsection 2.3 for the definition).

Theorem 1.1 (Hitczenko, McConnell). Let $X$ be a Banach space, $1 \leq p<\infty$. Then $X$ is $U M D$ if and only if for any $X$-valued tangent martingale difference sequences $\left(d_{n}\right)_{n \geq 1}$ and $\left(e_{n}\right)_{n \geq 1}$ one has that

$$
\mathbb{E} \sup _{0 \leq N<\infty}\left\|\sum_{n=1}^{N} d_{n}\right\|^{p} \bar{\sim}_{p, X} \mathbb{E} \sup _{0 \leq N<\infty}\left\|\sum_{n=1}^{N} e_{n}\right\|^{p} .
$$

(Note that the paper [73] did not cover the case $p=1$, and [44] was never published. Nevertheless, the reader can find this case in [25, pp. 424-425] and in Theorem 5.9).

A classical example of tangent martingale different sequences is provided by independent mean zero random variables. Let $\left(\xi_{n}\right)_{n \geq 1}$ be real-valued mean zero independent random variables, let $\left(v_{n}\right)_{n \geq 1}$ be $X$-valued bounded predictable (i.e. $v_{n}$ depends only on $\left.\xi_{1}, \ldots, \xi_{n-1}\right)$. Then $\left(v_{n} \xi_{n}\right)_{n \geq 1}$ is a martingale difference sequence. Moreover, then $\left(v_{n} \xi_{n}^{\prime}\right)_{n \geq 1}$ is a tangent martingale difference sequence for $\left(\xi_{n}^{\prime}\right)_{n \geq 1}$ being an independent copy of $\left(\xi_{n}\right)_{n \geq 1}$ (see Example 2.28), so in the UMD case (1.2) yields

$$
\mathbb{E} \sup _{0 \leq N<\infty}\left\|\sum_{n=1}^{N} v_{n} \xi_{n}\right\|^{p} \bar{\sim}_{p, X} \mathbb{E} \sup _{0 \leq N<\infty}\left\|\sum_{n=1}^{N} v_{n} \xi_{n}^{\prime}\right\|^{p} .
$$

It turned out that (1.3) characterizes the UMD property if one sets $\left(\xi_{n}\right)_{n \geq 1}$ to be Rademachers $^{3}$ (see Bourgain [11] and Garling [36, 37]), Gaussians (see Garling [36] and McConnell [73]), or Poissons (see Proposition 3.23). In the Gaussian and Poisson cases the equivalence of (1.3) and the UMD property basically says that the following estimates hold for $X$-valued stochastic integrals

$$
\mathbb{E} \sup _{t \geq 0}\left\|\int_{0}^{t} \Phi \mathrm{d} W\right\|^{p} \bar{\sim}_{p, X} \mathbb{E} \sup _{t \geq 0}\left\|\int_{0}^{t} \Phi \mathrm{d} \widetilde{W}\right\|^{p}
$$

\footnotetext{
${ }^{1}$ See Subsection 2.2 for the definition of a conditional probability.

${ }^{2}$ I.e. the distributions $(1.1)$ are symmetric a.s., equivalently $\left(d_{n}\right),\left(-d_{n}\right),\left(e_{n}\right)$, and $\left(-e_{n}\right)$ are tangent altogether.

${ }^{3}$ See Definition 2.1 .
} 


$$
\mathbb{E} \sup _{t \geq 0}\left\|\int_{0}^{t} F \mathrm{~d} \widetilde{N}\right\|^{p} \bar{\sim}_{p, X} \mathbb{E} \sup _{t \geq 0}\left\|\int_{0}^{t} F \mathrm{~d} \widetilde{N}_{\text {ind }}\right\|^{p}
$$

(here $\Phi$ and $F$ are $X$-valued elementary predictable, $W$ is a Brownian motion, $\widetilde{N}$ is a compensated standard Poisson process, $\widetilde{W}$ and $\widetilde{N}_{\text {ind }}$ are independent copies of $W$ and $\widetilde{N}$ respectively), which allows one to change the driving Brownian or Poisson noise in a stochastic integral by an independent copy without losing the information about strong $L^{p}$-norms of the stochastic integral, are equivalent to your Banach space $X$ having the UMD property. Estimates of the form (1.4) turned out to be exceptionally important in vector-valued stochastic integration theory as the right-hand side of (1.4) is nothing but a $\gamma$-norm (see Subsection 2.11) of $\Phi$ which is a natural extension of the Hilbert-Schmidt norm to general Banach spaces (see McConnell [73] and van Neerven, Veraar, and Weis [81], see also [83, 106, 108] for a general continuous martingale case and Dirksen [30] for the Poisson case). Estimates (1.4) and (1.5) justify that tangent martingales are extremely important for vector-valued stochastic integration.

The procedure of changing the noise by an independent copy (in our case this was $\left.\left(\xi_{n}\right) \mapsto\left(\xi_{n}^{\prime}\right)\right)$ together with extending the filtration in the corresponding way (i.e. $\left.\mathcal{F}_{n}^{\prime}:=\sigma\left(\mathcal{F}_{n}, \xi_{1}^{\prime}, \ldots, \xi_{n}^{\prime}\right)\right)$ creates a special tangent martingale difference sequence, namely a decoupled one which can be defined in the following way: $\left(e_{n}\right)$ is a decoupled tangent martingale difference sequence to $\left(d_{n}\right)$ if $\left(e_{n}\right)$ are conditionally independent given $\mathcal{G}:=\sigma\left(\left(d_{n}\right)\right)$, i.e. for any Borel $B_{1}, \ldots, B_{N} \subset X$ a.s.

$$
\mathbb{P}\left(e_{1} \in B_{1}, \ldots, e_{N} \in B_{N} \mid \mathcal{G}\right)=\mathbb{P}\left(e_{1} \in B_{1} \mid \mathcal{G}\right) \cdot \ldots \cdot \mathbb{P}\left(e_{N} \in B_{N} \mid \mathcal{G}\right),
$$

and $\mathbb{P}\left(e_{n} \mid \mathcal{F}_{n-1}\right)=\mathbb{P}\left(e_{n} \mid \mathcal{G}\right)$ for any $n \geq 1$. Note that such a martingale difference sequence might not exist on the probability space with the original filtration, so one may need to extend the probability space and filtration in such a way that $\left(d_{n}\right)$ preserves its martingale property. Existence and uniqueness of such a decoupled $\left(e_{n}\right)$ was proved by Kwapień and Woyczyński in [64] (see also de la Peña [28], de la Peña and Giné [29], especially [29, Section 6.1] for a detailed proof, Kallenberg [58], and S.G. Cox and Geiss [24]). The goal of the present paper is to extend Theorem 1.1 to the continuous-time setting and to discover in this case the explicit form of a decoupled tangent local martingale.

Let us start with explaining what continuous-time tangent local martingales are. To this end we will need Lévy martingales. What do we know about them? Well, one of the most fundamental features of Lévy processes is the LévyKhinchin formula which is the case of a Lévy martingale $L$ with $L_{0}=0$ has the following form (see e.g. [52, 102])

$$
\mathbb{E} e^{i \theta L_{t}}=\exp \left\{t\left(-\frac{1}{2} \sigma^{2} \theta^{2}+\int_{\mathbb{R}} e^{i \theta x}-1-i \theta x \mathrm{~d} \nu(\mathrm{d} x)\right)\right\}, \quad t \geq 0, \quad \theta \in \mathbb{R},
$$

for some fixed $\sigma \geq 0$ and for some fixed measure $\nu$ on $\mathbb{R}$. It turns out that the pair $(\sigma, \nu)$ characterizes the distribution of a Lévy martingale, and it has the following analogue for a general real-valued martingale $M:\left(\left[M^{c}\right], \nu^{M}\right)$, where $M^{c}$ 
is the continuous part of $M$ (see Subsection 2.7) with $\left[M^{c}\right]$ being the quadratic variation of $M^{c}$ (see Subsection 2.6), and $\nu^{M}$ is a compensator of a random measure $\mu^{M}$ defined on $\mathbb{R}_{+} \times \mathbb{R}$ by

$$
\mu^{M}([0, t] \times B):=\sum_{0 \leq s \leq t} \mathbf{1}_{B \backslash\{0\}}\left(\Delta M_{t}\right), \quad t \geq 0, \quad B \in \mathcal{B}(\mathbb{R})
$$

(see Subsection 2.8). In the case $M=L$ we have that $\left[M^{c}\right]_{t}=\sigma^{2} t$ and $\nu^{M}=\lambda \otimes \nu$, where $\lambda$ is the Lebesgue measure on $\mathbb{R}_{+}$. This pair $\left(\left[M^{c}\right], \nu^{M}\right)$ is called to be the local characteristics (a.k.a. Grigelionis characteristics or Jacod-Grigelionis characteristics) of $M$, and two continuous-time local martingales are called tangent if their local characteristics coincide. Continuous-times tangent martingales and local characteristics were intensively studied by Jacod [48, 49, 50], Jacod and Shiryaev [52], Jacod and Sadi [51], Kwapień and Woyczyński [62, 63, 64, 65], and Kallenberg [58] (see also [73, 81, 85, 86]). In particular, Kallenberg proved in [58] that for any real-valued continuous-time tangent martingales $M$ and $N$ one has that

$$
\mathbb{E} \sup _{t \geq 0}\left|M_{t}\right|^{p} \bar{\sim}_{p} \mathbb{E} \sup _{t \geq 0}\left|N_{t}\right|^{p}, \quad 1 \leq p<\infty,
$$

with more general inequalities (including concave functions of moderate growth) under additional assumptions on $M$ and $N$ (e.g. conditional symmetry). Furthermore, in $[50,51,58,64]$ it was shown that any real-valued martingale $M$ has a decoupled tangent local martingale $N$, i.e. a tangent local martingale $N$ defined on an enlarged probability space with an enlarged filtration such that $N(\omega)$ is a martingale with independent increments and with local characteristics $\left(\left[M^{c}\right](\omega), \nu^{M}(\omega)\right)$ for a.e. $\omega \in \Omega$ from the original probability space. Moreover, in the quasi-left continuous setting it was shown in $[50,51,64]$ that such a martingale can be obtained via the following procedure: if we discretize $M$ on $[0, T]$, i.e. consider a discrete martingale $\left(f_{k}^{n}\right)_{k=1}^{n}=\left(M_{T k / n}\right)_{k=1}^{n}$, and consider a decoupled tangent martingale $\tilde{f}^{n}:=\left(\tilde{f}_{k}^{n}\right)_{k=1}^{n}$, then $\tilde{f}^{n}$ converges in distribution to $N$ as random variables with values in the Skorokhod space $\mathcal{D}([0, T], \mathbb{R})$ (see Definition 2.2) as $n \rightarrow \infty$. This in particular justifies the definition of a continuous-time decoupled tangent martingale.

In the present paper we are going to explore various facts concerning vectorvalued continuous-time tangent martingales. We will mainly focus on the following three questions:

- How do local characteristics look like in Banach spaces?

- What is a decoupled tangent martingale in this case?

- Can we extend decoupling inequalities (1.8) to infinite dimensions?

We will also try to answer all the supplementary and related problems appearing while working on these three questions. Let us outline the structure of the paper section-by-section.

In Section 2 we present some preliminaries to the paper, i.e. certain assertions (e.g. concerning martingales, random measures, stochastic integration, et cetera) which we will heavily need throughout the paper. 
Our main Section 3 is devoted to the definition of vector-valued continuoustime tangent martingales, basic $L^{p}$-estimates for these martingales, and the construction of a decoupled tangent martingale. How do we define tangent martingales in the vector-valued case? As we saw in Theorem 1.1, a Banach space $X$ having the UMD property plays an important rôle for existence of $L^{p}$-bounds for discrete tangent martingales. This also turned out to be equivalent to existence of local characteristics of a general $X$-valued martingale $M$. Namely, due to $[116,118] X$ has the UMD property if and only if a general $X$-valued martingale $M$ has the Meyer-Yoeurp decomposition, i.e. it can be uniquely decomposed into a sum of a continuous local martingale $M^{c}$ and a purely discontinuous local martingale $M^{d}$ (see Remark 2.19). In this case we define the local characteristics of $M$ to be the pair $\left(\llbracket M^{c} \rrbracket, \nu^{M}\right)$, where $\llbracket M^{c} \rrbracket$ is a covariation bilinear form, i.e. a symmetric bilinear form-valued process satisfying

$$
\llbracket M^{c} \rrbracket_{t}\left(x^{*}, x^{*}\right)=\left[\left\langle M, x^{*}\right\rangle\right]_{t}, \quad t \geq 0,
$$

for any $x^{*} \in X^{*}$ a.s. (such a process exists because of Remark 2.13), and $\nu^{M}$ is a compensator of a random measure $\mu^{M}$ defined on $\mathbb{R}_{+} \times X$ analogously to (1.7) (see Subsection 2.6 and 2.8). Similarly to the real-valued case, two $X$-valued martingales are tangent if they have the same local characteristics.

Next, we present $L^{p}$-estimates for UMD-valued tangent martingales. In Theorem 3.7 we extend the result (1.8) of Kallenberg to any UMD Banach space $X$, i.e. we prove that for any UMD Banach space $X$ and for any $X$-valued tangent martingales $M$ and $N$ one has that

$$
\mathbb{E} \sup _{t \geq 0}\left\|M_{t}\right\|^{p} \bar{\sim}_{p, X} \mathbb{E} \sup _{t \geq 0}\left\|N_{t}\right\|^{p}, \quad 1 \leq p<\infty
$$

Let us say a couple of words about how do we gain (1.9). To this end we need the canonical decomposition. Thanks to Meyer [76] and Yoeurp [120] any realvalued martingale $M$ can be uniquely decomposed into a sum of a continuous local martingale $M^{c}$ (the Wiener-like part), a purely discontinuous quasi-left continuous local martingale $M^{q}$ (the Poisson-like part), and a purely discontinuous local martingale $M^{a}$ with accessible jumps (the discrete-like part). It turned out that this decomposition can be expanded to the vector-valued case if and only if $X$ has the UMD property (see $[116,118]$ ). Moreover, as it is shown in Subsection 3.2 if $M=M^{c}+M^{q}+M^{a}$ and $N=N^{c}+N^{q}+N^{a}$ are the canonical decompositions of tangent martingales $M$ and $N$, then $M^{i}$ and $N^{i}$ are tangent for any $i \in\{c, q, a\}$, and thus by strong $L^{p}$-estimates for the canonical decomposition presented in [119] (see Theorem 2.18) we need to show (1.9) separately for each of these three cases. Then the continuous case immediately follows from weak differential subordination inequalities obtained in [91, 116, 119] and the discrete-like case can be shown via a standard discretization trick (see Subsection B.1) and Theorem 1.1.

The most complicated and the most interesting mathematically is the Poissonlike case. First we show that (1.5) holds true not just for a compensated Poisson process, but for any stochastic integral with respect to a Poisson random 
measure (see Proposition 3.23). Next we prove that any UMD-valued quasi-left continuous purely discontinuous martingale can be presented as a stochastic integral with respect to a quasi-left continuous compensated random measure (see Theorem 3.30). Finally, by exploiting a certain approximation argument, we may assume that this random measure is defined over a finite jump space, and hence this is a time-changed Poisson random measure thanks to a fundamental result by Meyer [77] and Papangelou [92] (see e.g. also [1, 12, 56]) which says that any quasi-left continuous integer random measure after a certain time change becomes a Poisson random measure. As this time change depends only on the compensator measure (which is one of local characteristics and which is the same for $M^{q}$ and $N^{q}$ ), (1.5) immediately yields (1.9) for the quasi-left continuous purely discontinuous case.

Another highlight point of Section 3 is existence, uniqueness, and construction of a decoupled tangent martingale. First, in Theorem 3.8 we extend the result of Jacod [50], Kwapień and Woyczyński [64], and Kallenberg [58] on existence of a decoupled tangent martingale to general UMD-valued martingales (recall that they have shown this existence only in the real-valued case). Next in Subsection 3.8 we show that a decoupled tangent martingale is unique in distribution (which extends the discrete case, see [29, 64]). Finally, in Subsection 3.9 we prove that if $N$ is a decoupled tangent martingale of $M$, then $N$ has independent increments given the local characteristics $\left(\llbracket M^{c} \rrbracket, \nu^{M}\right)$ of $M$ which e.g. generalizes [58, Theorem 3.1].

It is of interest to take a closer look at the structure of tangent martingales. Let us consider a particular case of (1.4) and (1.5). Intuitively it seems that stochastic integrals $\int \Phi \mathrm{d} \widetilde{W}$ and $\int F \mathrm{~d} \widetilde{N}_{\text {ind }}$ occurring in (1.4) and (1.5) should be decoupled tangent martingales to $\int \Phi \mathrm{d} W$ and $\int F \mathrm{~d} \widetilde{N}$ respectively. And this is true as $\int \Phi(\omega) \mathrm{d} \widetilde{W}$ is a.s. a martingale with independent increments and with the local characteristics $\left(\Phi(\omega) \Phi^{*}(\omega), 0\right)$ (here we can consider $\Phi \in \mathcal{L}\left(L^{2}\left(\mathbb{R}_{+}\right), X\right)$ instead of $\Phi: \mathbb{R}_{+} \rightarrow X$ a.s. as $\Phi$ is elementary predictable, see Subsection 2.10 and Section 6), and $\int F(\omega) \mathrm{d} \widetilde{N}_{\text {ind }}$ has a.s. independent increments and the local characteristics $\left(0, \nu^{F}(\omega)\right)$ with the measure $\nu^{F}(\omega)$ defined on $\mathbb{R}_{+} \times X$ by

$$
\nu^{F}(\omega)([0, t] \times B)=\int_{0}^{t} \mathbf{1}_{B}(F(s, \omega)) \mathrm{d} s, \quad t \geq 0, \quad B \in \mathcal{B}(X) .
$$

For a general martingale we have an expanded version the this construction. Recall that for a given UMD Banach space $X$ any $X$-valued martingale $M$ has the canonical decomposition $M=M^{c}+M^{q}+M^{a}$. Let us present a corresponding decoupled tangent martingale $N^{c}, N^{q}$, and $N^{a}$ for each of the cases separately (in the end we can simply sum up $N:=N^{c}+N^{q}+N^{a}$ these cases, see Subsection 3.7). It turns out that by Subsection 3.3 we have that $M^{c} \circ \tau^{c}=\int \Phi \mathrm{d} W_{H}$ for some time-change $\tau^{c}$, some Hilbert space $H$, some $H$-cylindrical Brownian motion $W_{H}$ (see Subsection 2.10), and some $\Phi: \Omega \rightarrow \gamma\left(L^{2}\left(\mathbb{R}_{+} ; H\right), X\right.$ ) (see Subsection 2.11; we are allowed to integrate such functions due to [81]). Then it is sufficient to set $N^{c}:=\int \Phi \mathrm{d} \widetilde{W}_{H} \circ A^{c}$ (where $A^{c}$ is the inverse time change to $\tau$, i.e. $\tau \circ A_{t}=A \circ \tau_{t}=t$ a.s. for any $\left.t \geq 0\right)$ to be the corresponding decoupled 
tangent martingale $N^{c}$ to $M^{c}$ for some independent $H$-cylindrical Brownian motion $\widetilde{W}_{H}$. Therefore $N^{c}(\omega)$ is a time-changed Wiener integral with a deterministic integrator, which agrees with (1.4). The construction of a decoupled tangent martingale $N^{a}$ to $M^{a}$ simply copies the one done in the discrete case due to the approximation argument presented in Proposition B.1 (see [28, 29, 64, 65] and Subsection 3.6).

The most intriguing thing happens in the quasi-left continuous case. Recall that $M^{q}$ can be presented as an integral with respect to a compensated random measure, namely

$$
M_{t}^{q}=\int_{[0, t] \times X} x \mathrm{~d} \bar{\mu}^{M^{q}}(\cdot, x), \quad t \geq 0,
$$

where $\mu^{M^{q}}$ is defined by $(1.7), \nu^{M^{q}}$ is the corresponding compensator, $\bar{\mu}^{M^{q}}=$ $\mu^{M^{q}}-\nu^{M^{q}}$ (see Theorem 3.30). It turns out that in this case

$$
N_{t}^{q}:=\int_{[0, t] \times X} x \mathrm{~d} \bar{\mu}_{\text {Cox }}^{M^{q}}(\cdot, x), \quad t \geq 0,
$$

is a decoupled tangent martingale to $M^{q}$, where $\mu_{\mathrm{Cox}}^{M^{q}}(\cdot, x)$ is a Cox process directed by $\nu^{M^{q}}, \bar{\mu}_{\mathrm{Cox}}^{M^{q}}=\mu_{\mathrm{Cox}}^{M^{q}}-\nu^{M^{q}}$. Cox processes were introduced by D.R. Cox in [22], and in the present case this is a random measure on an enlarged probability space such that $\mu_{\text {Cox }}^{M^{q}}(\omega)$ is a Poisson random measure on $\mathbb{R}_{+} \times X$ with the intensity (or compensator, see Subsection 2.9) $\nu^{M^{q}}(\omega)$ for a.e. $\omega \in \Omega$ from the original probability space. Thus $N^{q}(\omega)$ is a Poisson integral with deterministic integrator, which corresponds to (1.5). The idea of employing Cox processes for creating decoupled tangent processes here is not new (see e.g. [58]), but what is the most difficult in the vector-valued case is to show that both integrals (1.10) and (1.11) make sense and tangent (see Subsection 3.5).

It is worth noticing that in Subsection 3.4 we are discussing $L^{p}$-estimates for general vector-valued integrals with respect to general random measures. Recall that this type of estimates goes back to Novikov [84], where he upper bounded an $L^{p}$-moment of a real-valued stochastic integral $\int F \mathrm{~d} \bar{\mu}$ by integrals in terms of $F$ and the compensator $\nu$ of $\mu$ (here $\bar{\mu}=\mu-\nu$; see Lemma 3.4). Later on sharp estimates of this form have been proven by Marinelli and Röckner [71] in the Hilbert space case and by Dirksen and the author [32] in the $L^{q}$ case $(1<q<\infty)$. In Theorem 3.22 we show that for any UMD-valued elementary predictable $F$ and for any quasi-left continuous random measure $\mu$ one has that

$$
\mathbb{E} \sup _{t \geq 0}\left\|\int_{[0, t] \times J} F \mathrm{~d} \bar{\mu}\right\|^{p} \bar{\sim}_{p, X} \mathbb{E}\left\|\int_{\mathbb{R}_{+} \times J} F \mathrm{~d} \bar{\mu}_{\mathrm{Cox}}\right\|^{p}, \quad 1 \leq p<\infty,
$$

where $\nu$ is a compensator of $\mu, \bar{\mu}:=\mu-\nu, \mu_{\text {Cox }}$ is a Cox process directed by $\nu$, and $\bar{\mu}_{\mathrm{Cox}}:=\mu_{\mathrm{Cox}}-\nu$. Note that though it seems that the right-hand side of (1.12) depends on $F$ and $\mu_{\mathrm{Cox}}$, the distribution of the Cox process entirely depends on $\nu$ (in particular, $\mu_{\mathrm{Cox}}(\omega)$ is a Poisson random measure with the intensity $\nu(\omega))$, and so on the right-hand side of (1.12) we in fact have $\mathbb{E}\|F\|_{p, X, \nu}^{p}$, where $\|F(\omega)\|_{p, X, \nu(\omega)}$ is the $L^{p}$-norm of a stochastic integral of a deterministic 
function $F(\omega)$ with respect to the corresponding compensated Poisson random measure (see Subsection 2.9 and $[2,3]$ ). Thus even though (1.12) does not provide an explicit formula for a stochastic integral in terms of $F$ and $\nu$, as it was done in [32, 71, 84], nevertheless it semigeneralizes the papers [32, 71, 84] as it tells us that in order to get $L^{p}$ bounds for UMD-valued stochastic integrals with respect to a general random measure we need only to prove the corresponding estimates for the Poisson case with deterministic integrands (see e.g. Remark 3.26).

In Section 4 we show that if $X$ satisfies the so-called decoupling property (e.g. if $X=L^{1}$ ), then inequalities of the form

$$
\mathbb{E} \sup _{0 \leq t \leq T}\left\|M_{t}\right\|^{p} \lesssim_{p, X} \mathbb{E}\left\|N_{T}\right\|^{p}, \quad T>0, \quad p \in[1, \infty),
$$

are possible for an $X$-valued martingale $M$ satisfying broad assumptions (see e.g. Remark 6.5), where $N$ is a corresponding decoupled tangent local martingale. Recall that the decoupling property was introduced by S.G. Cox and Veraar in $[25,26]$ as a natural property while working with discrete decoupled tangent martingales and stochastic integrals.

In [58] Kallenberg also has shown $\phi$-inequalities for tangent continuous martingales (where $\phi$ is a convex function of moderate growth; recall that one can even omit the convexity assumption for conditionally symmetric martingales). In Section 5 we extend these inequalities to full generality (i.e. general martingales in UMD Banach spaces). Though [58] also treats the semimartingale case, it is not known to the author how to prove such inequalities for vector-valued semimartingales.

In Section 6 we present estimates for vector-valued stochastic integrals with respect to a general martingale which extend both (1.4) and (1.5). Namely, we show that for a general $H$-valued martingale $\widetilde{M}$ (where $H$ is a Hilbert space) and an $\mathcal{L}(H, X)$-valued elementary predictable process $\Phi$ one has that for any $1 \leq p<\infty$

$$
\begin{aligned}
& \sup _{t \geq 0}\left\|\int_{0}^{t} \Phi \mathrm{d} \widetilde{M}\right\|^{p} \bar{\sim}_{p, X} \mathbb{E}\left\|\Phi q_{\widetilde{M}^{c}}^{1 / 2}\right\|_{\gamma\left(L^{2}\left(\mathbb{R}_{+},\left[M^{c}\right] ; H\right), X\right)}^{p} \\
&+\mathbb{E}\left\|\int_{\mathbb{R}_{+} \times H} \Phi(s) h \mathrm{~d} \bar{\mu}_{\operatorname{Cox}}^{\widetilde{M}^{q}}(s, h)\right\|^{p} \\
&+\mathbb{E}\left\|\sum_{0 \leq t<\infty} \Phi \Delta \widetilde{N}_{t}^{a}\right\|^{p}
\end{aligned}
$$

where $\widetilde{M}=\widetilde{M}^{c}+\widetilde{M}^{q}+\widetilde{M}^{a}$ is the canonical decomposition, $q_{\widetilde{M}^{c}}$ is a quadratic variation derivative of $\widetilde{M}^{c}$ (see Subsection 2.6), and $\widetilde{N}^{a}$ is a decoupled tangent martingale to $\widetilde{M}^{a}$. Note that the right-hand side of (1.14) in fact can be seen as an $L^{p}$ moment of a predictable process. Such estimates are in the spirit of works of Novikov [84] and Dirksen and the author in [32], and they are very different from the classical vector-valued Burkholder-Davis-Gundy inequalities presented 
e.g. in $[21,72,109,119]$. Note that the upper bound of (1.14) characterizes the decoupling property (see Section 4 and Remark 6.5).

As it was discussed above, the notion of tangency heavily exploits the MeyerYoeurp decomposition, which existence for a general $X$-valued martingale is equivalent to $X$ obtaining the UMD property. But what if we have weak tangency, i.e. what if for a given Banach space $X$ and a pair of $X$-valued martingales $M$ and $N$ we have that $\left\langle M, x^{*}\right\rangle$ and $\left\langle N, x^{*}\right\rangle$ are tangent for any $x^{*} \in X^{*}$ ? How does this correspond to the tangency property and will we then have $L^{p_{-}}$ estimates for a family of Banach spaces different from the UMD one? In Section 7 we show that in the UMD case weak tangency and tangency coincide. Moreover, in the non-UMD setting no estimate of the form (1.9) for weakly tangent martingales is possible.

In Section 8 we discuss for which Banach spaces it is possible to extend the definition of decoupled tangent local martingales (and prove their existence) via using weak local characteristics. It turns out that this is possible for Banach spaces with the so-called recoupling property which is dual to the decoupling property (1.13) and which occurs to be equivalent to the well-discovered $U M D^{+}$ property. Moreover, the converse holds true, i.e. a Banach space $X$ having the recoupling property is necessary for any $X$-valued local martingale to have a decoupled tangent local martingale (see Theorem 8.6 and Remark 8.7). It remains open whether recoupling and UMD are identical (see e.g. [46, Section O]).

In Section 9 we consider vector-valued martingales with independent increments. First recall that one of the inventors of local characteristics was Grigelionis (that is why local characteristics are sometimes called Grigelionis characteristics). In particular, in [42] he proved that a real-valued martingale has independent increments if and only if it has deterministic local characteristics (this result was extended by Jacod and Shiryaev in [52] to multi dimensions). In Section 9 we extend this celebrated result to infinite dimensions. In preliminary Subsection 9.1 we show that for any Banach space $X$, an $X$-valued local martingale $M$ has independent increments if and only if it has deterministic weak local characteristics, i.e. the family $\left(\left[\left\langle M, x^{*}\right\rangle^{c}, \nu^{\left\langle M, x^{*}\right\rangle}\right]\right)_{x^{*} \in X^{*}}$ is deterministic (such an object always exists since $\left\langle M, x^{*}\right\rangle$ has local characteristics as a real-valued local martingale). Next in Subsection 9.2 we prove that if this is the case, then $M$ actually has local characteristics (which are of course deterministic), and moreover, $M$ has the canonical decomposition $M=M^{c}+M^{q}+M^{a}$ so that $M^{c}, M^{q}$, and $M^{a}$ are mutually independent, and there exists a deterministic time-change $\tau^{c}$ such that $M^{c} \circ \tau^{c}=\int \Phi \mathrm{d} W_{H}$ is a stochastic integral of some deterministic $\Phi \in \gamma\left(L^{2}\left(\mathbb{R}_{+} ; H\right), X\right)$ with respect to some $H$-cylindrical Brownian motion $W_{H}, M^{q}=\int x \mathrm{~d} \widetilde{N}(\cdot, x)$ for some fixed Poisson random measure $N$ on $\mathbb{R}_{+} \times X$, and $M^{a}$ is a sum of its independent jumps which occur at deterministic family of times $\left(t_{n}\right)_{n \geq 1}$. Note that throughout Section $9 X$ is a general Banach space and there is no need in the UMD property.

Recall that Jacod [50] and Kwapień and Woyczyński [64] proved that for a real-valued quasi-left continuous martingale $M$ a decoupled tangent martingale $N$ on $[0, T]$ is nothing but a limit in distribution of discrete decoupled tangent 
martingales $\tilde{f}^{n}$ as $n \rightarrow \infty$, where for each $n \geq 1$ a martingale $\tilde{f}^{n}:=\left(\tilde{f}_{k}^{n}\right)_{k=1}^{n}$ is a decoupled tangent martingale to a discrete martingale $\left(f_{k}^{n}\right)_{k=1}^{n}=\left(M_{T k / n}\right)_{k=1}^{n}$, and the limit is considered as a limit in distribution of random variables with values in the Skorokhod space $\mathcal{D}([0, T], \mathbb{R})$ (see Definition 2.2). In Section 10 we extend this result to general UMD-valued martingales (thus somehow mixing together the discrete works of McConnell [73], Hitczenko [44], and de la Peña [28] and quasi-left continuous works of Jacod $[49,50]$ and Kwapień and Woyczyński [64]). In our setting such a limit theorem is possible since we know what the limiting object is (i.e. how does a decoupled tangent martingale look like) due to Section 3, because of certain approximation techniques, and thanks to properties of stochastic integrals and the canonical decomposition.

Section 11 is devoted to a characterization of the local characteristics of a general UMD-valued martingale via an exponential formula which can be considered as an extension of the Lévy-Khinchin formula. There we show that for any UMD-valued martingale $M$ with the local characteristics $\left(\llbracket M^{c} \rrbracket, \nu^{M}\right)$ and for any $x^{*} \in X^{*}$

$$
t \mapsto e^{i\left\langle M_{t}, x^{*}\right\rangle} / G_{t}\left(x^{*}\right), \quad t \geq 0,
$$

is a local martingale on $\left[0, \tau_{G\left(x^{*}\right)}\right)$, where

$$
\begin{gathered}
A_{t}\left(x^{*}\right):=-\frac{1}{2} \llbracket M^{c} \rrbracket_{t}\left(x^{*}, x^{*}\right)+\int_{[0, t] \times X}\left(e^{i\left\langle x, x^{*}\right\rangle}-1-i\left\langle x, x^{*}\right\rangle\right) \mathrm{d} \nu^{M}(s, x), \quad t \geq 0, \\
G_{t}\left(x^{*}\right):=e^{A_{t}\left(x^{*}\right)} \Pi_{0 \leq s \leq t}\left(1+\Delta A_{s}\left(x^{*}\right)\right) e^{-\Delta A_{s}\left(x^{*}\right)}, \quad t \geq 0,
\end{gathered}
$$

and $\tau_{G\left(x^{*}\right)}:=\inf \left\{t \geq 0: G_{t}\left(x^{*}\right)=0\right\}=\inf \left\{t \geq 0: \Delta A_{t}\left(x^{*}\right)=-1\right\}$. Moreover, $\left(\llbracket M^{c} \rrbracket, \nu^{M}\right)$ are unique bilinear form-valued predictable process and predictable random measure such that $(1.15)$ is a local martingale on $\left[0, \tau_{G\left(x^{*}\right)}\right)$. This is a natural generalization of the Lévy-Khinchin formula (1.6) as if we set $M$ to be quasi-left continuous with independent increments, then $\tau_{G\left(x^{*}\right)}=\infty$ and $G\left(x^{*}\right)$ is deterministic, and consequently (1.15) being a local martingale implies (1.6). The proof of the fact that (1.15) is a local martingale on $\left[0, \tau_{G\left(x^{*}\right)}\right)$ presented in Section 11 follows directly from the multidimensional case shown by Jacod and Shiryaev in [52].

In Section 12 we discover $L^{p}$-inequalities for characteristically subordinated and characteristically dominated martingales. These notions are predictable versions of weak differential subordination of martingales (see [91, 115, 116, 118]) and martingale domination (see $[19,89,119]$ ) and have the following form: for a Banach space $X$ an $X$-valued martingale $N$ is characteristically subordinate to an $X$-valued martingale $M$ if for any $x^{*} \in X^{*}$ we have that a.s.

(i) $\left|\left\langle N_{0}, x^{*}\right\rangle\right| \leq\left|\left\langle M_{0}, x^{*}\right\rangle\right|$,

(ii) $\left[\left\langle N^{c}, x^{*}\right\rangle\right]_{t}-\left[\left\langle N^{c}, x^{*}\right\rangle\right]_{s} \leq\left[\left\langle M^{c}, x^{*}\right\rangle\right]_{t}-\left[\left\langle M^{c}, x^{*}\right\rangle\right]_{s}$ for any $0 \leq s \leq t$, and (iii) $\nu^{\left\langle N, x^{*}\right\rangle} \leq \nu^{\left\langle M, x^{*}\right\rangle}$,

and $N$ is characteristically dominated by $M$ if a.s.

(i) $\left|\left\langle N_{0}, x^{*}\right\rangle\right| \leq\left|\left\langle M_{0}, x^{*}\right\rangle\right|$ for any $x^{*} \in X^{*}$, 
(ii) $\llbracket N^{c} \rrbracket_{\infty} \leq \llbracket M^{c} \rrbracket_{\infty}$, and

(iii) $\nu^{N}\left(\mathbb{R}_{+} \times \cdot\right) \leq \nu^{M}\left(\mathbb{R}_{+} \times \cdot\right)$

(here $M^{c}$ and $N^{c}$ are the continuous parts of $M$ and $N$, see Subsection 2.7). In Subsection 12.1 we compare weak differential subordination and characteristic subordination (these properties turn out to be incomparable) and show inequalities (1.9) for characteristically subordinated martingales. In Subsection 12.2 we show inequalities (1.9) for quasi-left continuous characteristically dominated martingales (both estimates are proven in the UMD setting). $L^{p_{-}}$ estimates for general characteristically dominated martingales remain open (see Remark 12.10) as the author does not know how to gain such estimates in the discrete case, though this case is very much in the spirit of the original work of Zinn [122].

In the end of the present paper we have appendix Sections A and B where we collect some technical facts concerning tangency and martingale approximations.

All over this section we used to talk about some mysterious UMD spaces. Recall that UMD spaces were introduced by Burkholder in 1980's while working with martingale transforms (see e.g. $[15,16,17,20]$ ), and nowadays these spaces are used abundantly in vector-valued stochastic and harmonic analysis (see e.g. $[11,39,46,81,101,115,119])$. Let us shortly outline here where exactly the UMD property is needed/used in the present paper.

- Theorem 1.1 due to Hitczenko and McConnell,

- Burkholder's works $[15,17]$ on martingale transforms,

- existence of the Meyer-Yoeurp and the canonical decomposition and the corresponding $L^{p}$ - and $\phi$-estimates (see $[116,118,119]$ ),

- vector-valued stochastic integration with respect to a cylindrical Brownian noise thanks to van Neerven, Veraar, and Weis [81],

- Burkholder-Davis-Gundy inequalities (see [109, 119]),

- existence of a covariation bilinear form $\llbracket M \rrbracket$ (see [119]).

On the other hand, we obtain several new characterizations of the UMD property, such as

- estimate (1.9) for continuous-time tangent martingales,

- existence of a decoupled tangent martingale (see Theorem 3.8 and Section 8),

- estimate (1.5),

- the fact that for a purely discontinuous quasi-left continuous martingale $M^{q}$ the integral $\int x \mathrm{~d} \bar{\mu}^{M^{q}}(\cdot, x)$ exists and coincides with $M^{q}$ (see Theorem 3.30),

- estimate (1.12),

- $L^{p}$-estimates for characteristically subordinated and characteristically dominated martingales (see Section 12).

This demonstrates once again that the UMD property is not just a technical assumption, but a key player in any game involving martingales in Banach spaces. 


\section{Preliminaries}

Throughout the present article any Banach space is considered to be over the scalar field $\mathbb{R}$. (This is done as we are going to work with continuous-time martingales, which properties are well discovered only in the case of the real scalar field, see e.g. $[52,56,95]$.)

Let $X$ be a Banach space, $B \subset X$ be Borel. Then we denote the $\sigma$-algebra of all Borel subsets of $B$ by $\mathcal{B}(B)$.

For $a, b \in \mathbb{R}$ we write $a \lesssim_{A} b$ if there exists a constant $c$ depending only on $A$ such that $a \leq c b . \gtrsim_{A}$ is defined analogously. We write $a{\sim_{A}} b$ if both $a \lesssim_{A} b$ and $a \gtrsim_{A} b$ hold simultaneously.

We will need the following definitions.

Definition 2.1. A random variable $\xi: \Omega \rightarrow \mathbb{R}$ is called Rademacher if $\mathbb{P}(\xi=$ 1) $=\mathbb{P}(\xi=-1)=1 / 2$.

Definition 2.2. Let $X$ be a Banach space, $A \in \mathbb{R}$ be an interval (finite or infinite). The linear space $\mathcal{D}(A, X)$ of all $X$-valued càdlàg (i.e. right continuous with left limits) functions on $A$ is called the Skorokhod space.

Recall that $\mathcal{D}(A, X)$ endowed with the sup-norm is a Banach space (see e.g. $[105,115])$.

For a Banach space $X$ and for a measurable space $(S, \Sigma)$ a function $f: S \rightarrow$ $X$ is called strongly measurable if there exists a sequence $\left(f_{n}\right)_{n \geq 1}$ of simple functions such that $f_{n} \rightarrow f$ pointwise on $S$ (see [46, Section 1.1]). In the sequel we will call a function $f$ strongly predictable if it is strongly measurable with respect to the predictable $\sigma$-algebra (which is either $\mathcal{P}$, see Subsection 2.5, or $\widetilde{\mathcal{P}}$, see Subsection 2.8, depending on the underlying $S$ ).

For a Banach space $X$ and a function $A: \mathbb{R}_{+} \rightarrow X$ we set $A^{*} \in \overline{\mathbb{R}}_{+}$to be $A^{*}:=\sup _{t \geq 0}\left\|A_{t}\right\|$.

Throughout the paper, unless stated otherwise, the probability space and filtration are assumed to be generated by all the processes involved.

\subsection{Enlargement of a filtered probability space}

We will need the following definition of an enlargement of a filtered probability space (see e.g. [64, pp. 172-174]).

Definition 2.3. Let $(\Omega, \mathcal{F}, \mathbb{P})$ be a probability space with a filtration $\mathbb{F}=\left(\mathcal{F}_{t}\right)_{t \geq 0}$. Then a probability space $(\bar{\Omega}, \overline{\mathcal{F}}, \overline{\mathbb{P}})$ with a filtration $\overline{\mathbb{F}}=\left(\overline{\mathcal{F}}_{t}\right)_{t \geq 0}$ is called to be an enlargement of $(\Omega, \mathcal{F}, \mathbb{P})$ and $\mathbb{F}$ if there exists a measurable space $(\widehat{\Omega}, \widehat{\mathcal{F}})$ such that $\bar{\Omega}=\Omega \times \widehat{\Omega}$ and $\overline{\mathcal{F}}=\mathcal{F} \otimes \widehat{\mathcal{F}}$, if there exists a family of probability measures $\left(\widehat{\mathbb{P}}_{\omega}\right)_{\omega \in \Omega}$ such that $\omega \mapsto \widehat{\mathbb{P}}_{\omega}(B)$ is $\mathcal{F}$-measurable for any $B \in \widehat{\mathcal{F}}$ and

$$
\overline{\mathbb{P}}(A \times B)=\int_{A} \widehat{\mathbb{P}}_{\omega}(B) \mathrm{d} \mathbb{P}(\omega), \quad A \in \mathcal{F}, B \in \widehat{\mathcal{F}},
$$


and if for any $\omega \in \Omega$ there exists a filtration $\widehat{\mathbb{F}}^{\omega}=\left(\widehat{\mathcal{F}}_{t}^{\omega}\right)_{t \geq 0}$ such that for any $B \in \widehat{\mathcal{F}}$ the process

$$
(t, \omega) \mapsto \mathbf{1}_{\widehat{\mathcal{F}}_{t}^{\omega}}(B), \quad t \geq 0, \omega \in \Omega,
$$

is $\mathbb{F}$-adapted, and such that $\overline{\mathcal{F}}_{t}=\mathcal{F}_{t} \otimes \widehat{\mathcal{F}}_{t}$ for any $t \geq 0$, i.e.

$$
A \times B \in \overline{\mathcal{F}}_{t} \text { if } A \in \mathcal{F}_{t} \text { and } B \in \widehat{\mathcal{F}}_{t}^{\omega} \text { for any } \omega \in A .
$$

Example 2.4. A classical example of an enlargement of a filtered probability space can be a product space, i.e. the case when $\widehat{\mathbb{P}}_{\omega}=\widehat{\mathbb{P}}_{\text {and }} \widehat{\mathcal{F}}_{t}^{\omega}=\widehat{\mathcal{F}}_{t}, t \geq 0$, for any $\omega \in \Omega$ for some fixed measure $\widehat{\mathbb{P}}$ and some fixed filtration $\widehat{\mathbb{F}}=\left(\widehat{\mathcal{F}}_{t}\right)_{t \geq 0}$.

\subsection{Conditional expectation on a product space. Conditional probability and conditional independence}

Let $(\Omega, \mathcal{F}, \mathbb{P})$ be a probability space, and assume that there exist probability spaces $\left(\Omega^{\prime}, \mathcal{F}^{\prime}, \mathbb{P}^{\prime}\right)$ and $\left(\Omega^{\prime \prime}, \mathcal{F}^{\prime \prime}, \mathbb{P}_{\omega^{\prime}}^{\prime \prime}\right)_{\omega^{\prime} \in \Omega^{\prime}}\left(\right.$ where $\mathbb{P}_{\omega^{\prime}}^{\prime \prime}$ depends on $\omega^{\prime} \in \Omega^{\prime}$ in $\mathcal{F}^{\prime}$-measurable way, see Subsection 2.1) such that

$$
(\Omega, \mathcal{F}, \mathbb{P})=\left(\Omega^{\prime} \times \Omega^{\prime \prime}, \mathcal{F}^{\prime} \otimes \mathcal{F}^{\prime \prime}, \mathbb{P}^{\prime} \otimes \mathbb{P}^{\prime \prime}\right),
$$

i.e. $\mathbb{P}_{\omega^{\prime}}^{\prime \prime}\left(A_{2}\right)$ is $\mathcal{F}^{\prime}$-measurable for any $A_{2} \in \mathcal{F}^{\prime \prime}$ and

$$
\mathbb{P}\left(A_{1} \times A_{2}\right)=\int_{A_{1}} \mathbb{P}_{\omega^{\prime}}^{\prime \prime}\left(A_{2}\right) \mathrm{d} \mathbb{P}^{\prime}\left(\omega^{\prime}\right), \quad A_{1} \in \mathcal{F}^{\prime}, \quad A_{2} \in \mathcal{F}^{\prime \prime} .
$$

A particular example would be if $\mathbb{P}_{\omega^{\prime}}^{\prime \prime}=\mathbb{P}^{\prime \prime}$ is a probability measure which does not depend on $\omega^{\prime} \in \Omega^{\prime}$. Let $X$ be a Banach space, and let $f \in L^{1}(\Omega ; X)$ (see [46, Section 1.2] for the definition of $\left.L^{p}(\Omega ; X)\right)$. Then $\mathbb{E}\left(f \mid \mathcal{F}^{\prime}\right)$ is well defined (see [46, Section 2.6]; by $\mathbb{E}\left(\cdot \mid \mathcal{F}^{\prime}\right)$ here we mean $\left.\mathbb{E}\left(\cdot \mid \mathcal{F}^{\prime} \otimes\left\{\Omega^{\prime \prime}, \varnothing\right\}\right)\right)$, and moreover, by Fubini's theorem $f\left(\omega^{\prime}, \cdot\right)$ exists and strongly measurable for a.e. $\omega^{\prime} \in \Omega^{\prime}$ (the proof is analogous to the one provided by [9, Section 3.4]). It is easy to see that for a.e. $\omega^{\prime} \in \Omega^{\prime}$

$$
\mathbb{E}\left(f \mid \mathcal{F}^{\prime}\right)\left(\omega^{\prime}, \cdot\right)=\int_{\Omega^{\prime \prime}} f\left(\omega^{\prime}, \omega^{\prime \prime}\right) \mathrm{d} \mathbb{P}_{\omega^{\prime}}^{\prime \prime}\left(\omega^{\prime \prime}\right)=: \mathbb{E}_{\Omega^{\prime \prime}} f\left(\omega^{\prime}, \cdot\right),
$$

where the notation $\mathbb{E}_{\Omega^{\prime \prime}}$ means averaging for every fixed $\omega^{\prime} \in \Omega^{\prime}$ over $\Omega^{\prime \prime}$. Indeed, for any $A \in \mathcal{F}^{\prime}$ by Fubini's theorem we have that

$$
\int_{A \times \Omega^{\prime \prime}} f \mathrm{~d} \mathbb{P}=\int_{A} \mathbb{E}_{\Omega^{\prime \prime}} f\left(\omega^{\prime}, \cdot\right) \mathrm{d} \mathbb{P}^{\prime}\left(\omega^{\prime}\right),
$$

so (2.2) follows by the definition of a conditional expectation.

Example 2.5. If there exists an $\mathcal{F}^{\prime \prime}$-measurable $\xi: \Omega^{\prime \prime} \rightarrow \mathbb{R}$ such that $\mathcal{F}^{\prime \prime}=$ $\sigma(\xi)$ for a.e. $\omega^{\prime} \in \Omega^{\prime}$, then we will often write $\mathbb{E}_{\xi}:=\mathbb{E}_{\Omega^{\prime \prime}}=\mathbb{E}\left(\cdot \mid \mathcal{F}^{\prime}\right)$ (i.e. averaging over all the values of $\xi$ ). 
Let $(\Omega, \mathcal{F}, \mathbb{P})$ be a probability space, $(S, \Sigma)$ be a measurable space, $\xi: \Omega \rightarrow S$ be a random variable. Let $\mathcal{G} \subset \mathcal{F}$ be a sub- $\sigma$-algebra. Then we define the conditional probability $\mathbb{P}(\xi \mid \mathcal{G}): \Sigma \rightarrow L^{1}(\Omega)$ to be as follows

$$
\mathbb{P}(\xi \mid \mathcal{G})(A):=\mathbb{E}\left(\mathbf{1}_{A}(\xi) \mid \mathcal{G}\right), \quad A \in \Sigma .
$$

Now let $N \geq 1,\left(\xi_{n}\right)_{n=1}^{N}$ be $S$-valued random variables. Then $\xi_{1}, \ldots, \xi_{N}$ are called conditionally independent given $\mathcal{G}$ if for any sets $B_{1}, \ldots, B_{N} \in \Sigma$ we have that

$$
\mathbb{P}\left(\left(\xi_{n}\right)_{n=1}^{N} \mid \mathcal{G}\right)\left(B_{1} \times \cdots \times B_{N}\right)=\Pi_{n=1}^{N} \mathbb{P}\left(\xi_{n} \mid \mathcal{G}\right)\left(B_{n}\right) .
$$

In the sequel we will need the following proposition.

Proposition 2.6. Let $(\Omega, \mathcal{F}, \mathbb{P})$ be defined by (2.1) for some $\left(\Omega^{\prime}, \mathcal{F}^{\prime}, \mathbb{P}^{\prime}\right)$ and for some family $\left(\Omega^{\prime \prime}, \mathcal{F}^{\prime \prime}, \mathbb{P}_{\omega^{\prime}}^{\prime \prime}\right)_{\omega^{\prime} \in \Omega^{\prime}}$. Let $\left(\xi_{n}\right)_{n=1}^{N}$ be as above. Assume that for almost any fixed $\omega^{\prime} \in \Omega^{\prime},\left(\xi_{n}\left(\omega^{\prime}, \cdot\right)\right)_{n=1}^{N}$ are independent. Then $\left(\xi_{n}\right)_{n=1}^{N}$ are conditionally independent given $\mathcal{F}^{\prime}$.

Proof. By the definition of conditional independence we need to show that for any sets $B_{1}, \ldots, B_{n} \in \Sigma$

$$
\mathbb{P}\left(\left(\xi_{n}\right)_{n=1}^{N} \mid \mathcal{F}^{\prime}\right)\left(B_{1} \times \cdots \times B_{N}\right)=\Pi_{n=1}^{N} \mathbb{P}\left(\xi_{n} \mid \mathcal{F}^{\prime}\right)\left(B_{n}\right) .
$$

To this end note that by $(2.2)$ for $\mathbb{P}^{\prime}$-a.e. $\omega^{\prime} \in \Omega^{\prime}$

$$
\begin{aligned}
\mathbb{P}\left(\left(\xi_{n}\right)_{n=1}^{N} \mid \mathcal{F}^{\prime}\right)\left(B_{1} \times \cdots \times B_{N}\right) & \left(\omega^{\prime}, \cdot\right)=\mathbb{E}\left(\mathbf{1}_{B_{1} \times \cdots \times B_{N}}\left(\xi_{1}, \ldots, \xi_{N}\right) \mid \mathcal{F}^{\prime}\right)\left(\omega^{\prime}, \cdot\right) \\
= & \int_{\Omega^{\prime \prime}} \Pi_{n=1}^{N} \mathbf{1}_{B_{n}}\left(\xi_{n}\left(\omega^{\prime}, \omega^{\prime \prime}\right)\right) \mathbb{P}_{\omega^{\prime}}^{\prime \prime}\left(\omega^{\prime \prime}\right) \\
= & \Pi_{n=1}^{N} \int_{\Omega^{\prime \prime}} \mathbf{1}_{B_{n}}\left(\xi_{n}\left(\omega^{\prime}, \omega^{\prime \prime}\right)\right) d \mathbb{P}_{\omega^{\prime}}^{\prime \prime}\left(\omega^{\prime \prime}\right) \\
= & \Pi_{n=1}^{N} \mathbb{P}\left(\xi_{n} \mid \mathcal{F}^{\prime}\right)\left(\omega^{\prime}, \cdot\right)\left(B_{n}\right),
\end{aligned}
$$

which terminates the proof.

We will also need the following consequence of the proposition.

Corollary 2.7. Let $(S, \Sigma)$ and $(T, \mathcal{T})$ be measurable spaces, let $(\Omega, \mathcal{F}, \mathbb{P})$ be defined by (2.1), and let $\xi: \Omega^{\prime} \rightarrow S$ and $\eta: \Omega \rightarrow T$ be measurable. Assume that $\eta$ is measurable with respect to $\sigma(\xi) \otimes \mathcal{F}^{\prime \prime}$. Let $F_{1}, \ldots, F_{N}: S \times T \rightarrow \mathbb{R}$. Then $\left(F_{n}(\xi, \eta)\right)_{n=1}^{N}$ are conditionally independent given $\sigma(\xi)$ if there exists $A \in \Sigma$ with $\mathbb{P}(\xi \in A)=1$ such that $\left(F_{n}(a, \eta(a, \cdot))\right)_{n=1}^{N}$ are independent for any $a \in A$. Proof. The corollary follows from Proposition 2.6 if one sets $\Omega^{\prime}:=A, \mathbb{P}^{\prime}:=\mathcal{L}(\xi)$, and

$$
\mathbb{P}_{\omega^{\prime}}^{\prime \prime}:=\mathcal{L}\left(\eta\left(\omega^{\prime}\right)\right), \quad \omega^{\prime} \in \Omega^{\prime},
$$

where the latter exists by [33, Theorem 10.2.2 and pp. 344, 386] (here $\mathcal{L}$ means the distribution).

We refer the reader to [46] for further details on vector-valued integration and vector-valued conditional expectation. 


\subsection{The UMD property}

A Banach space $X$ is called a $U M D^{4}$ space if for some (equivalently, for all) $p \in(1, \infty)$ there exists a constant $\beta>0$ such that for every $N \geq 1$, every martingale difference sequence $\left(d_{n}\right)_{n=1}^{N}$ in $L^{p}(\Omega ; X)$, and every $\{-1,1\}$-valued sequence $\left(\varepsilon_{n}\right)_{n=1}^{N}$ we have

$$
\left(\mathbb{E}\left\|\sum_{n=1}^{N} \varepsilon_{n} d_{n}\right\|^{p}\right)^{\frac{1}{p}} \leq \beta\left(\mathbb{E}\left\|\sum_{n=1}^{N} d_{n}\right\|^{p}\right)^{\frac{1}{p}} .
$$

The least admissible constant $\beta$ is denoted by $\beta_{p, X}$ and is called the $U M D_{p}$ constant of $X$ (or just the UMD constant of $X$ if the value of $p$ is understood). It is well known (see [46, Chapter 4]) that $\beta_{p, X} \geq p^{*}-1$ and that $\beta_{p, H}=p^{*}-1$ for a Hilbert space $H$ and any $1<p<\infty$ (here $\left.p^{*}:=\max \{p, p /(p-1)\}\right)$.

We will also frequently use the following equivalent definition of the UMD property. $X$ is UMD if and only if for any $1 \leq p<\infty$ and for any $\left(d_{n}\right)_{n=1}^{N}$ and $\left(\varepsilon_{n}\right)_{n=1}^{N}$ as above we have that

$$
\mathbb{E} \sup _{1 \leq m \leq N}\left\|\sum_{n=1}^{m} \varepsilon_{n} d_{n}\right\|^{p} \bar{\sim}_{p, X} \mathbb{E} \sup _{1 \leq m \leq N}\left\|\sum_{n=1}^{m} d_{n}\right\|^{p} .
$$

Note that a similar definition of the UMD property can be provided for a general convex function of moderate growth (see e.g. [15, p. 1000]). We refer the reader to $[15,20,29,39,40,46,47,68,94,101,119]$ for details on UMD Banach spaces.

\subsection{Stopping times}

A stopping time $\tau$ is called predictable if there exists a sequence of stopping times $\left(\tau_{n}\right)_{n>1}$ such that $\tau_{n}<\tau$ a.s. on $\{\tau>0\}$ and $\tau_{n} \nearrow \tau$ a.s. as $n \rightarrow \infty$. A stopping time $\tau$ is called totally inaccessible if $\mathbb{P}(\tau=\sigma \neq \infty)=0$ for any predictable stopping time $\sigma$.

With a predictable stopping time $\tau$ we associate a $\sigma$-field $\mathcal{F}_{\tau-}$ which has the following form

$$
\mathcal{F}_{\tau-}:=\sigma\left\{\mathcal{F}_{0} \cup\left(\mathcal{F}_{t} \cap\{t<\tau\}\right), t>0\right\}=\sigma\left\{\mathcal{F}_{\tau_{n}}, n \geq 1\right\},
$$

where $\left(\tau_{n}\right)_{n \geq 1}$ is a sequence of stopping time announcing $\tau$ (see [56, p. 491] for details).

Later on we will work with different types of martingales based on the properties of their jumps, and in particular we will frequently use the following definition (see e.g. Subsection 2.7). Recall that for a càdlàg process $A$ and for a stopping time $\tau$ we set $\Delta A_{\tau}:=A_{\tau}-\lim _{\varepsilon \backslash 0} A_{0 \vee(\tau-\varepsilon)}$ on $\{\tau<\infty\}$.

Definition 2.8. Let $X$ be a Banach space, $A: \mathbb{R}_{+} \times \Omega \rightarrow X$ be a càdlàg process. Then $A$ is called quasi-left continuous if $\Delta A_{\tau}=0$ a.s. on $\{t<\infty\}$ for any predictable stopping time $\tau$. $A$ is called to have accessible jumps if $\Delta A_{\tau}=0$ a.s. on $\{t<\infty\}$ for any totally inaccessible stopping time $\tau$.

\footnotetext{
${ }^{4} \mathrm{UMD}$ stands for unconditional martingale differences.
} 
We refer the reader to $[32,52,56,116,118]$ for further details.

\subsection{Martingales: real- and Banach space-valued}

Let $(\Omega, \mathcal{F}, \mathbb{P})$ be a probability space with a filtration $\mathbb{F}=\left(\mathcal{F}_{t}\right)_{t \geq 0}$ which satisfies the usual conditions (see $[52,56,95])$. Then particularly $\mathbb{F}$ is right-continuous. A predictable $\sigma$-algebra $\mathcal{P}$ is a $\sigma$-algebra on $\mathbb{R}_{+} \times \Omega$ generated by all predictable rectangles of the form $(s, t] \times B$, where $0 \leq s<t$ and $B \in \mathcal{F}_{s}$.

Let $X$ be a Banach space. An adapted process $M: \mathbb{R}_{+} \times \Omega \rightarrow X$ is called a martingale if $M_{t} \in L^{1}(\Omega ; X)$ and $\mathbb{E}\left(M_{t} \mid \mathcal{F}_{s}\right)=M_{s}$ for all $0 \leq s \leq t . M$ is called a local martingale if there exists a nondecreasing sequence $\left(\tau_{n}\right)_{n \geq 1}$ of stopping times such that $\tau_{n} \nearrow \infty$ a.s. as $n \rightarrow \infty$ and $M^{\tau_{n}}$ is a martingale for any $n \geq 1$ (recall that for a stopping time $\tau$ we set $M_{t}^{\tau}:=M_{\tau \wedge t}, t \geq 0$, which is a local martingale given $M$ is a local martingale, see $[52,56,95])$. It is well known that in the real-valued case any local martingale is càdlàg (i.e. has a version which is right-continuous and that has limits from the left-hand side). The same holds for a general $X$-valued local martingale $M$ as well (see e.g. $[105,115]$ ), so for any stopping time $\tau$ one can define $\Delta M_{\tau}:=M_{\tau}-\lim _{\varepsilon \backslash 0} M_{0 \vee(\tau-\varepsilon)}$ on $\{\tau<\infty\}$.

Let $1 \leq p \leq \infty$. A martingale $M: \mathbb{R}_{+} \times \Omega \rightarrow X$ is called an $L^{p}$-bounded martingale if $M_{t} \in L^{p}(\Omega ; X)$ for each $t \geq 0$ and there exists a limit $M_{\infty}:=$ $\lim _{t \rightarrow \infty} M_{t} \in L^{p}(\Omega ; X)$ in $L^{p}(\Omega ; X)$-sense.

Since $\|\cdot\|: X \rightarrow \mathbb{R}_{+}$is a convex function, and $M$ is a martingale, $\|M\|$ is a submartingale by Jensen's inequality, and hence by Doob's inequality (see e.g. [60, Theorem 1.3.8(i)]) we have that for all $1 \leq p<\infty$

$$
\mathbb{E}\left\|M_{t}\right\|^{p} \leq \mathbb{E} \sup _{0 \leq s \leq t}\left\|M_{s}\right\|^{p} \leq \frac{p}{p-1} \mathbb{E}\left\|M_{t}\right\|^{p}, \quad t \geq 0 .
$$

In fact, the following theorem holds for martingales having strong $L^{p}$-moments (see e.g. $[110,111]$ for the real-valued case, the infinite dimensional case can be proven analogously, see e.g. [32, 105, 115, 116, 117, 119]). Recall that Skorokhod spaces were defined in Definition 2.2.

Theorem 2.9. Let $X$ be a Banach space, $1 \leq p<\infty$. Then the family of all martingales $M: \mathbb{R}_{+} \times \Omega \rightarrow X$ satisfying $\mathbb{E} \sup _{t \geq 0}\left\|M_{t}\right\|^{p}<\infty$ forms a closed subspace of $L^{p}\left(\Omega ; \mathcal{D}\left(\mathbb{R}_{+}, X\right)\right)$.

Remark 2.10. Recall that any local martingale $M: \mathbb{R}_{+} \times \Omega \rightarrow X$ is locally in $L^{1}\left(\Omega ; \mathcal{D}\left(\mathbb{R}_{+}, X\right)\right)$. Indeed, set $\left(\tau_{n}\right)_{n \geq 1}$ be a localizing sequence and for each $n \geq 1$ set $\sigma_{n}:=\inf \left\{t \geq 0:\left\|M_{t}\right\| \geq n\right\}$. Then $\sigma_{n} \rightarrow \infty$ as $n \rightarrow \infty$ a.s. since $M$ has càdlàg paths, and thus $\tau_{n} \wedge \sigma_{n} \wedge n \rightarrow \infty$ as $n \rightarrow \infty$ a.s. as well. On the other hand we have that for each $n \geq 1$

$$
\begin{aligned}
\mathbb{E} \sup _{t \geq 0}\left\|M_{t}^{\tau_{n} \wedge \sigma_{n} \wedge n}\right\| & =\mathbb{E} \sup _{0 \leq t \leq \tau_{n} \wedge \sigma_{n} \wedge n}\left\|M_{t}\right\| \leq \mathbb{E} n \wedge\left\|M_{\tau_{n} \wedge \sigma_{n} \wedge n}\right\| \\
& \leq n \wedge \mathbb{E}\left\|M_{\tau_{n} \wedge \sigma_{n} \wedge n}\right\|=\leq n \wedge \mathbb{E}\left\|M_{n}^{\tau_{n} \wedge \sigma_{n}}\right\|<\infty
\end{aligned}
$$

where we used the fact that $M^{\tau_{n} \wedge \sigma_{n}}$ is a martingale as $M^{\tau_{n}}$ is a martingale (see e.g. [56]). 
Later we will need the following lemma proven e.g. in [32, Subsection 5.3] (see also $[56,119])$.

Lemma 2.11. Let $X$ be a Banach space, $M: \mathbb{R}_{+} \times \Omega \rightarrow X$ be a martingale such that $\lim _{\sup _{t \rightarrow \infty}} \mathbb{E}\left\|M_{t}\right\|<\infty$. Let $\tau$ be a finite predictable stopping time. Then $\Delta M_{\tau}$ is integrable and

$$
\mathbb{E}\left(\left.\Delta M_{\tau}\right|_{\mathcal{F}_{\tau-}}\right)=0
$$

where $\mathcal{F}_{\tau-}$ is defined by (2.5). Equivalently, $t \mapsto \Delta M_{\tau} \mathbf{1}_{[\tau, \infty)}(t), t \geq 0$, is a martingale.

We refer the reader to $[46,56,74,75,90,94,95,105,117]$ for further information on martingales.

\subsection{Quadratic variation}

Let $H$ be a Hilbert space, $M: \mathbb{R}_{+} \times \Omega \rightarrow H$ be a local martingale. We define a quadratic variation of $M$ in the following way:

$$
[M]_{t}:=\mathbb{P}-\lim _{\operatorname{mesh} \rightarrow 0} \sum_{n=1}^{N}\left\|M\left(t_{n}\right)-M\left(t_{n-1}\right)\right\|^{2},
$$

where the limit in probability is taken over partitions $0=t_{0}<\ldots<t_{N}=$ $t$. Note that $[M]$ exists and is nondecreasing a.s. The reader can find more information on quadratic variations in $[74,75,108]$ for the vector-valued setting, and in $[56,75,95]$ for the real-valued setting.

As it was shown in [78, Proposition 1] (see also [100, Theorem 2.13] and [108, Example 3.19] for the continuous case), for any $H$-valued martingale $M$ there exists an adapted process $q_{M}: \mathbb{R}_{+} \times \Omega \rightarrow \mathcal{L}(H)$ which we will call a quadratic variation derivative, such that the trace of $q_{M}$ does not exceed 1 on $\mathbb{R}_{+} \times \Omega$, $q_{M}$ is self-adjoint nonnegative on $\mathbb{R}_{+} \times \Omega$, and for any $h, g \in H$ a.s.

$$
[\langle M, h\rangle,\langle M, g\rangle]_{t}=\int_{0}^{t}\left\langle q_{M}^{1 / 2}(s) h, q_{M}^{1 / 2}(s) g\right\rangle \mathrm{d}[M]_{s}, \quad t \geq 0 .
$$

For any martingales $M, N: \mathbb{R}_{+} \times \Omega \rightarrow H$ we can define a covariation $[M, N]$ : $\mathbb{R}_{+} \times \Omega \rightarrow \mathbb{R}$ as $[M, N]:=\frac{1}{4}([M+N]-[M-N])$. Since $M$ and $N$ have càdlàg versions, $[M]$ and $[M, N]$ have càdlàg versions as well (see [52, Theorem I.4.47] and $[56,74])$.

Definition 2.12. Let $X$ be a Banach space, $M: \mathbb{R}_{+} \times \Omega \rightarrow X$ be a local martingale. Fix $t \geq 0$. Then $M$ is said to have a covariation bilinear from $\llbracket M \rrbracket_{t}$ at $t \geq 0$ if there exists a continuous bilinear form-valued random variable $\llbracket M \rrbracket_{t}: X^{*} \times X^{*} \times \Omega \rightarrow \mathbb{R}$ such that for any fixed $x^{*}, y^{*} \in X^{*}$ a.s. $\llbracket M \rrbracket_{t}\left(x^{*}, y^{*}\right)=$ $\left[\left\langle M, x^{*}\right\rangle,\left\langle M, y^{*}\right\rangle\right]_{t}$. 
Remark 2.13. It is known due to [119] that if $X$ has the UMD property, then any $X$-valued local martingale $M$ has a covariation bilinear form $\llbracket M \rrbracket$. Moreover, $\llbracket M \rrbracket$ has a càdlàg adapted version, and if $M$ is continuous, then $\llbracket M \rrbracket$ has a continuous version as well, and for a general local martingale $M$ one has that $\gamma\left(\llbracket M \rrbracket_{t}\right)<\infty$ a.s., where for a bilinear form $V: X^{*} \times X^{*} \rightarrow X$ we set the Gaussian characteristic $\gamma(V)$ to be

- the $L^{2}$-norm of a Gaussian random variable $\xi$ having $V$ as its bilinear covariance form, i.e. $\mathbb{E}\left\langle\xi, x^{*}\right\rangle\left\langle\xi, y^{*}\right\rangle=V\left(x^{*}, y^{*}\right)$ for any $x^{*}, y^{*} \in X^{*}$, if such $\xi$ exists,

- $\infty$, if such $\xi$ does not exist.

We refer the reader to [119] for further details.

\subsection{The canonical decomposition}

In this subsection we discuss the so-called canonical decomposition of martingales. First let us start with the following technical definitions. Recall that a càdlàg function $A: \mathbb{R}_{+} \rightarrow X$ is called pure jump if $A_{t}=A_{0}+\sum_{0<s \leq t} \Delta A_{s}$ for any $t \geq 0$, where the latter sum converges absolutely.

Definition 2.14. Let $X$ be a Banach space. A local martingale $M: \mathbb{R}_{+} \times \Omega \rightarrow X$ is called purely discontinuous if $\left[\left\langle M, x^{*}\right\rangle\right]$ is pure jump a.s. for any $x^{*} \in X^{*}$.

Definition 2.15. Let $X$ be a Banach space, $M: \mathbb{R}_{+} \times \Omega \rightarrow X$ be a local martingale. Then $M$ is called to have the canonical decomposition if there exist local martingales $M^{c}, M^{q}, M^{a}: \mathbb{R}_{+} \times \Omega \rightarrow X$ such that $M^{c}$ is continuous, $M^{q}$ is purely discontinuous quasi-left continuous, $M^{a}$ is purely discontinuous with accessible jumps, $M_{0}^{c}=M_{0}^{q}=0$ a.s., and $M=M^{c}+M^{q}+M^{a}$.

Remark 2.16. Note that if $M=M^{c}+M^{q}+M^{a}$ is the canonical decomposition, then $\left\langle M, x^{*}\right\rangle=\left\langle M^{c}, x^{*}\right\rangle+\left\langle M^{q}, x^{*}\right\rangle+\left\langle M^{a}, x^{*}\right\rangle$ is the canonical decomposition for any $x^{*} \in X^{*}$ (see e.g. [32, 116, 118]).

Remark 2.17. Note that by [52, 56, 116, 118] if the canonical decomposition of a local martingale $M$ exists, then $M^{q}$ and $M^{a}$ collect different jumps of $M$, i.e. a.s.

$$
\begin{aligned}
& \left\{t \geq 0: \Delta M_{t}^{q} \neq 0\right\} \cup\left\{t \geq 0: \Delta M_{t}^{a} \neq 0\right\}=\left\{t \geq 0: \Delta M_{t} \neq 0\right\} \\
& \left\{t \geq 0: \Delta M_{t}^{q} \neq 0\right\} \cap\left\{t \geq 0: \Delta M_{t}^{a} \neq 0\right\}=\varnothing .
\end{aligned}
$$

Then the following theorem holds, which was first proved in $[76,120]$ in the real-valued case, and in $[116,118,119]$ in the vector-valued case (see also [56, Chapter 25]).

Theorem 2.18 (The canonical decomposition). Let $X$ be a Banach space. Then $X$ is UMD if and only if any local martingale $M: \mathbb{R}_{+} \times \Omega \rightarrow X$ has the canonical decomposition $M=M^{c}+M^{q}+M^{a}$. Moreover, if this is the case, then the canonical decomposition is unique, and for any $1 \leq p<\infty$

$$
\mathbb{E} \sup _{t \geq 0}\left\|M_{t}^{c}\right\|^{p}+\mathbb{E} \sup _{t \geq 0}\left\|M_{t}^{q}\right\|^{p}+\mathbb{E} \sup _{t \geq 0}\left\|M_{t}^{a}\right\|^{p} \bar{\sim}_{p, X} \mathbb{E} \sup _{t \geq 0}\left\|M_{t}\right\|^{p} .
$$


If we will have a closer look on each of the parts of the canonical decomposition, then we will figure out that $M^{c}$ is in fact a time changed stochastic integral with respect to a cylindrical Brownian motion (see Subsection 3.3), $M^{q}$ is a time changed stochastic integral with respect to a Poisson random measure (see Subsection 2.9), while $M^{a}$ can be represented as a discrete martingale if it has finitely many jumps (see Subsection 3.6 and B.1; see also $[32,56,116]$ ). Thus we often call $M^{c}$ the Wiener-like part, $M^{q}$ the Poisson-like part, while $M^{a}$ is often called a discrete-like part of $M$ : in many cases the corresponding techniques help in finding required inequalities for $M^{c}, M^{q}$, and $M^{a}$.

Note that the canonical decomposition plays an important rôle in stochastic integration theory (see e.g. $[31,32,119]$ ).

Remark 2.19. Often we will use the so-called Meyer-Yoeurp decomposition which splits a local martingale $M$ into a continuous part $M^{c}$ and a purely discontinuous part $M^{d}$. This decomposition is unique if it exists, and in the case of existence of the canonical decomposition $M=M^{c}+M^{q}+M^{a}$ one obviously has $M^{d}=M^{q}+M^{a}$. Analogously to Theorem 2.18 one can show that for a given Banach space X every X-valued local martingale has the Meyer-Yoeurp decomposition if and only if $X$ has the UMD property (see [116, 117, 118]).

Later we will need the following lemma shown in [32, Subsection 5.1] (see [56] for the real-valued version). Recall that two stopping times $\tau$ and $\sigma$ have disjoint graphs if $\mathbb{P}(\tau=\sigma<\infty)=0$.

Lemma 2.20. Let $X$ be a Banach space, $M: \mathbb{R}_{+} \times \Omega \rightarrow X$ be a purely discontinuous local martingale with accessible jumps. Then there exist a sequence $\left(\tau_{n}\right)_{n \geq 1}$ of finite predictable stopping times with disjoint graphs such that a.s.

$$
\left\{t \geq 0: \Delta M_{t} \neq 0\right\} \subset\left\{\tau_{1}, \ldots, \tau_{n}, \ldots\right\} .
$$

\subsection{Random measures}

Let $(J, \mathcal{J})$ be a measurable space so that $\mathcal{J}$ is countably generated. A family

$$
\mu=\{\mu(\omega ; \mathrm{d} t, \mathrm{~d} x), \omega \in \Omega\}
$$

of nonnegative measures on $\left(\mathbb{R}_{+} \times J, \mathcal{B}\left(\mathbb{R}_{+}\right) \otimes \mathcal{J}\right)$ is called a random measure. A random measure $\mu$ is called integer-valued if it takes values in $\mathbb{N} \cup\{\infty\}$, i.e. for each $A \in \mathcal{B}\left(\mathbb{R}_{+}\right) \otimes \mathcal{J}$ one has that $\mu(A) \in \mathbb{N} \cup\{\infty\}$ a.s., and if $\mu(\{t\} \times J) \in\{0,1\}$ a.s. for all $t \geq 0$ (so $\mu$ is a sum of atoms with a.s. disjoint supports, see [52, Proposition II.1.14]). We say that $\mu$ is non-atomic in time if $\mu(\{t\} \times J)=0$ a.s. for all $t \geq 0$.

Let $\mathcal{O}$ be the optional $\sigma$-algebra on $\mathbb{R}_{+} \times \Omega$, i.e. the $\sigma$-algebra generated by all càdlàg adapted processes. Let $\widetilde{\mathcal{O}}:=\mathcal{O} \otimes \mathcal{J}, \widetilde{\mathcal{P}}:=\mathcal{P} \otimes \mathcal{J}$ (see Subsection 2.5 for the definition of $\mathcal{P}$ ). A random measure $\mu$ is called optional (resp. predictable) if for any $\widetilde{\mathcal{O}}$-measurable (resp. $\widetilde{\mathcal{P}}$-measurable) nonnegative $F: \mathbb{R}_{+} \times \Omega \times J \rightarrow \mathbb{R}_{+}$ 
the stochastic integral

$$
(t, \omega) \mapsto \int_{\mathbb{R}_{+} \times J} \mathbf{1}_{[0, t]}(s) F(s, \omega, x) \mu(\omega ; \mathrm{d} s, \mathrm{~d} x), \quad t \geq 0, \omega \in \Omega,
$$

as a function from $\mathbb{R}_{+} \times \Omega$ to $\overline{\mathbb{R}}_{+}$is optional (resp. predictable).

Let $X$ be a Banach space. Then we can extend stochastic integration with respect to random measures to $X$-valued processes in the following way. Let $F$ : $\mathbb{R}_{+} \times \Omega \times J \rightarrow X$ be elementary predictable, i.e. there exists partition $B_{1}, \ldots, B_{N}$ of $J, 0=t_{0}<t_{1} \ldots<t_{L}$, and simple $X$-valued random variables $\left(\xi_{n, \ell}\right)_{n=1, m=1}^{N, L}$ such that $\xi_{n, \ell}$ is $\mathcal{F}_{t_{\ell-1}}$-measurable for any $1 \leq \ell \leq L$ and $1 \leq n \leq N$ and

$$
F(t, \cdot, j)=\sum_{n=1}^{N} \sum_{\ell=1}^{L} \mathbf{1}_{\left(t_{\ell-1}, t_{\ell}\right]}(t) \mathbf{1}_{B_{n}}(j) \xi_{n, \ell} .
$$

Let $\mu$ be a random measure. The integral

$$
\begin{aligned}
t \mapsto \int_{\mathbb{R}_{+} \times J} F(s, \cdot, x) \mathbf{1}_{[0, t]}(s) \mu(\cdot ; \mathrm{d} s, \mathrm{~d} x) & \\
& :=\sum_{n=1}^{N} \sum_{\ell=1}^{L} \mu\left(\left(t_{\ell-1} \wedge t, t_{\ell} \wedge t\right] \times B_{n}\right) \xi_{n, \ell}, \quad t \geq 0,
\end{aligned}
$$

is well-defined and optional (resp. predictable) if $\mu$ is optional (resp. predictable), and $\int_{\mathbb{R}_{+} \times J}\|F\| \mathrm{d} \mu$ is a.s. bounded.

A random measure $\mu$ is called $\widetilde{\mathcal{P}}$ - $\sigma$-finite if there exists an increasing sequence of sets $\left(A_{n}\right)_{n \geq 1} \subset \widetilde{\mathcal{P}}$ such that $\int_{\mathbb{R}_{+} \times J} \mathbf{1}_{A_{n}}(s, \omega, x) \mu(\omega ; \mathrm{d} s, \mathrm{~d} x)$ is finite a.s. and $\cup_{n} A_{n}=\mathbb{R}_{+} \times \Omega \times J$. According to [52, Theorem II.1.8] every $\widetilde{\mathcal{P}}-\sigma$-finite optional random measure $\mu$ has a compensator: a unique $\widetilde{\mathcal{P}}-\sigma$-finite predictable random measure $\nu$ such that

$$
\mathbb{E} \int_{\mathbb{R}_{+} \times J} F \mathrm{~d} \mu=\mathbb{E} \int_{\mathbb{R}_{+} \times J} F \mathrm{~d} \nu
$$

for each $\tilde{\mathcal{P}}$-measurable real-valued nonnegative $F$. For any optional $\widetilde{\mathcal{P}}$ - $\sigma$-finite measure $\mu$ we define the associated compensated random measure by $\bar{\mu}:=\mu-\nu$.

For each $\widetilde{\mathcal{P}}$-strongly-measurable $F: \mathbb{R}_{+} \times \Omega \times J \rightarrow X$ such that

$$
\mathbb{E} \int_{\mathbb{R}_{+} \times J}\|F\| \mathrm{d} \mu<\infty
$$

(or, equivalently, $\mathbb{E} \int_{\mathbb{R}_{+} \times J}\|F\| \mathrm{d} \nu<\infty$, see the definition of a compensator above) we can define a process

$$
t \mapsto \int_{[0, t] \times J} F \mathrm{~d} \bar{\mu}:=\int_{[0, t] \times J} F \mathrm{~d} \mu-\int_{[0, t] \times J} F \mathrm{~d} \nu, \quad t \geq 0,
$$

which turns out to be a purely discontinuous martingale (see Proposition 3.27, [52, Theorem II.1.8], and [32]).

We will need the following classical result of Novikov [84, Theorem 1]. 
Lemma 2.21 (A.A. Novikov). Let $\mu$ be an integer-valued optional random measure on $\mathbb{R}_{+} \times J$ with a compensator $\nu$ being non-atomic in time, $F: \mathbb{R}_{+} \times \Omega \times J \rightarrow$ $\mathbb{R}$ be $\widetilde{\mathcal{P}}$-measurable. Then

$$
\begin{array}{r}
\mathbb{E} \sup _{t \geq 0}\left|\int_{[0, t] \times J} f \mathrm{~d} \bar{\mu}\right|^{p} \lesssim p \mathbb{E} \int_{\mathbb{R}_{+} \times \Omega}|f|^{p} \mathrm{~d} \nu \text { if } 1 \leq p \leq 2, \\
\mathbb{E} \sup _{t \geq 0}\left|\int_{[0, t] \times J} f \mathrm{~d} \bar{\mu}\right|^{p} \lesssim p\left(\mathbb{E} \int_{\mathbb{R}_{+} \times J}|f|^{2} \mathrm{~d} \nu\right)^{\frac{p}{2}} \\
\quad+\mathbb{E} \int_{\mathbb{R}_{+} \times \Omega}|f|^{p} \mathrm{~d} \nu \text { if } p \geq 2 .
\end{array}
$$

For an $X$-valued martingale $M$ we associate a jump measure $\mu^{M}$ which is a random measure on $\mathbb{R}_{+} \times X$ that counts the jumps of $M$

$$
\mu^{M}([0, t] \times B):=\sum_{0 \leq s \leq t} \mathbf{1}_{B \backslash\{0\}}\left(\Delta M_{t}\right), \quad t \geq 0, \quad B \in \mathcal{B}(X) .
$$

Note that $\mu^{M}$ is $\widetilde{\mathcal{P}}$ - $\sigma$-finite and we will frequently use the following fact which was proved in [52, Corollary II.1.19] (see also [32, 56, 57]).

Lemma 2.22. Let $X$ be a Banach space, $M: \mathbb{R}_{+} \times \Omega \rightarrow X$ be a local martingale. Let $\mu^{M}$ be the associated jump measure. Then $M$ is quasi-left continuous if and only if the corresponding compensator $\nu^{M}$ of $\mu^{M}$ is non-atomic in time.

We refer the reader to $[32,41,50,52,56,57,69,71,84,85,119]$ for details on random measures and stochastic integration with respect to random measures.

\subsection{Poisson random measures}

An important example of random measures is a Poisson random measure.

Let $(S, \Sigma, \rho)$ be a measure space, $\rho$ be $\sigma$-finite. Then we can define a Poisson random measure (a.k.a. Poisson point process) $N_{\rho}$ with intensity (or compensator $) \rho$, i.e. a function $\Sigma \mapsto L^{0}\left(\Omega, \mathbb{N}_{0} \cup\{+\infty\}\right)$ satisfying the following properties

(i) $N_{\rho}(A)$ has the Poisson distribution with a parameter $\rho(A)$ for any $A \in \Sigma$ such that $\rho(A)<\infty$,

(ii) $N_{\rho}\left(A_{1}\right), \ldots, N_{\rho}\left(A_{n}\right)$ are independent for any disjoint $A_{1}, \ldots, A_{n} \in \Sigma$,

(iii) $N_{\rho}$ is a.s. a measure on $\Sigma$

(see [102, Chapter 4] and [61] for details). We can also define the compensated Poisson random measure $\widetilde{N}_{\rho}$ to be $\widetilde{N}_{\rho}(A):=N_{\rho}(A)-\rho(A)$ for any $A \in \Sigma$ satisfying $\rho(A)<\infty$.

Remark 2.23. If we set $S=\mathbb{R}_{+} \times J$ and $\rho=\nu=\lambda \otimes \nu_{0}$ (so that we have the setting which we used to work above) with $\lambda$ being the Lebesgue measure on $\mathbb{R}_{+}$ and $\nu_{0}$ being some fixed $\sigma$-finite measure on $J$, then we come up with Poisson measures that are often exploited as a noncontinuous noise for SPDE's (see e.g. [13, 30, 35, 41, 70, 84, 93, 121] and references therein). 
In the sequel we will need the following definition of an integral with respect to a Poisson random measure.

Definition 2.24. Let $X$ be a Banach space, $(S, \Sigma, \rho)$ be a measure space, $N_{\rho}$ be a Poisson random measure on $S$ with the intensity $\rho$. Then a strongly $\Sigma$ measurable function $F: S \rightarrow X$ is called integrable with respect to $\widetilde{N}_{\rho}=N_{\rho}-\rho$ if there exist an increasing family of sets $\left(A_{n}\right)_{n \geq 1} \in \Sigma$ such that $\cup_{A_{n}}=S$, $\int_{A_{n}}\|F\| \mathrm{d} \rho<\infty$, and $\int_{A_{n}} F \mathrm{~d} \widetilde{N}_{\rho}$ converges in $L^{1}(\Omega ; X)$ as $n \rightarrow \infty$.

Remark 2.25. Let $G: S \rightarrow X$ be strongly $\Sigma$-measurable such that $\int_{S}\|G\| \mathrm{d} \rho<$ $\infty$. Then $G \in L^{1}(S, \rho ; X)$, and as for any step function $H \in L^{1}(S, \rho ; X)$ we have that $\mathbb{E} \int_{S}\|H\| \mathrm{d} N_{\rho}=\|H\|_{L^{1}(S, \rho ; X)}$ by the definition of $N_{\rho}$ (in particular, $\mathbb{E} N_{\rho}(A)=\rho(A)$ for any $\left.A \in \Sigma\right)$, we can extend the stochastic integral definition to $G$ by a standard expanding operator procedure. Thus $\int_{A_{n}} F \mathrm{~d} \widetilde{N}_{\rho}:=$ $\int_{A_{n}} F \mathrm{~d} N_{\rho}-\int_{A_{n}} F \mathrm{~d} \rho$ in the definition above is well defined.

Remark 2.26. Definition 2.24 is quite different from the one given in Subsection 3.5 as we do not have a time scale (so there is no martingale structure) and since we are working with Poisson random measures. Moreover, notice that if such a family $\left(A_{n}\right)_{n \geq 1}$ exists, then for any other increasing family $\left(A_{n}^{\prime}\right)_{n \geq 1}$ having the same properties as $\left(A_{n}\right)_{n \geq 1}$ we will have that $\int_{A_{n}^{\prime}} F \mathrm{~d} \widetilde{N}_{\rho}$ converges in $L^{1}(\Omega ; X)$ as $n \rightarrow \infty$. Indeed, let

$$
\xi=\int_{S} F \mathrm{~d} \tilde{N}_{\rho}:=L^{1}(\Omega ; X)-\lim _{n \rightarrow \infty} \int_{A_{n}} F \mathrm{~d} \tilde{N}_{\rho}
$$

Then

$$
\left(\xi_{n}\right)_{n \geq 1}:=\left(\int_{A_{n}^{\prime}} F \mathrm{~d} \tilde{N}_{\rho}\right)_{n \geq 1},
$$

is a martingale with independent increments as $\xi_{n+1}-\xi_{n}=\int_{A_{n}^{\prime} \backslash A_{n-1}^{\prime}} F \mathrm{~d} \widetilde{N}_{\rho}$ is independent of $\sigma\left(\left.N_{\rho}\right|_{A_{n}^{\prime}}\right)$, and hence independent of $\xi_{1}, \ldots, \xi_{n}$. Thus we have that for any $x^{*} \in X^{*}, \mathbb{E}\left(\left\langle\xi, x^{*}\right\rangle \mid \sigma\left(\left.N_{\rho}\right|_{A_{n}^{\prime}}\right)\right)=\left\langle\xi_{n}, x^{*}\right\rangle$ for any $n \geq 1$ (which follows from the fact that

$$
\int_{A_{n}^{\prime} \cap A_{m}}\left\langle F, x^{*}\right\rangle \mathrm{d} \widetilde{N}_{\rho} \rightarrow \int_{A_{n}^{\prime}}\left\langle F, x^{*}\right\rangle \mathrm{d} \widetilde{N}_{\rho} \quad \text { in } \quad L^{1}(\Omega) \quad \text { as } \quad m \rightarrow \infty,
$$

from [46, Theorem 3.3.2], and from the definition (2.15) of $\xi$ ), so $\left\langle\xi_{n}, x^{*}\right\rangle$ converges to $\left\langle\xi, x^{*}\right\rangle$ by [46, Theorem 3.3.2], thus by the Itô-Nisio theorem [47, Theorem 6.4.1] we have that $\xi_{n}$ converges to $\xi$ in $L^{1}(\Omega ; X)$.

\subsection{Stochastic integration}

Let $H$ be a Hilbert space, $X$ be a Banach space. For each $x \in X$ and $h \in H$ we denote the linear operator $g \mapsto\langle g, h\rangle x, g \in H$, by $h \otimes x$. The process 
$\Phi: \mathbb{R}_{+} \times \Omega \rightarrow \mathcal{L}(H, X)$ is called elementary predictable with respect to the filtration $\mathbb{F}=\left(\mathcal{F}_{t}\right)_{t \geq 0}$ if it is of the form

$$
\Phi(t, \omega)=\sum_{k=1}^{K} \sum_{\ell=1}^{L} \mathbf{1}_{\left(t_{k-1}, t_{k}\right] \times B_{\ell k}}(t, \omega) \sum_{n=1}^{N} h_{n} \otimes x_{k \ell n}, \quad t \geq 0, \omega \in \Omega,
$$

where $0=t_{0}<\ldots<t_{K}<\infty$, for each $k=1, \ldots, K$ the sets $B_{1 k}, \ldots, B_{L k}$ are in $\mathcal{F}_{t_{k-1}}$, the vectors $h_{1}, \ldots, h_{N}$ are in $H$, and $\left(x_{k \ell n}\right)_{k, \ell, n=1}^{K, L}$ are elements of $X$. Let $\widetilde{M}: \mathbb{R}_{+} \times \Omega \rightarrow H$ be a local martingale. Then we define the stochastic integral $\Phi \cdot \widetilde{M}: \mathbb{R}_{+} \times \Omega \rightarrow X$ of $\Phi$ with respect to $\widetilde{M}$ as follows:

$$
(\Phi \cdot \widetilde{M})_{t}:=\sum_{k=1}^{K} \sum_{\ell=1}^{L} \mathbf{1}_{B_{\ell k}} \sum_{n=1}^{N}\left\langle\left(\widetilde{M}\left(t_{k} \wedge t\right)-\widetilde{M}\left(t_{k-1} \wedge t\right)\right), h_{n}\right\rangle x_{k \ell n}, \quad t \geq 0 .
$$

A map $W_{H}: \mathbb{R}_{+} \times H \rightarrow L^{2}(\Omega)$ is called an $H$-cylindrical Brownian motion (see [27, Chapter 4.1]) if

- $W_{H}(\cdot) h$ is a Brownian motion for any $h \in H$,

- $\mathbb{E} W_{H}(t) h W_{H}(s) g=\langle h, g\rangle \min \{t, s\}$ for all $h, g \in H$ and $t, s \geq 0$.

For an $H$-cylindrical Brownian motion $W_{H}$ we can define a stochastic integral of $\Phi$ of the form (2.16) in the following way

$$
\left(\Phi \cdot W_{H}\right)_{t}:=\sum_{k=1}^{K} \sum_{\ell=1}^{L} \mathbf{1}_{B_{k}} \sum_{n=1}^{N}\left(W_{H}\left(t_{k} \wedge t\right) h_{n}-W_{H}\left(t_{k-1} \wedge t\right) h_{n}\right) x_{k \ell n}, \quad t \geq 0 .
$$

Further, if $X=\mathbb{R}$, then due to [27, Theorem 4.12] (see also [56, 81, 108]) it is known that a.s.

$$
\left[\Phi \cdot W_{H}\right]_{t}=\int_{0}^{t}\|\Phi\|^{2} \mathrm{~d} s
$$

and in particular by the Burkholder-Davis-Gundy inequalities [56, Theorem 17.7] we have that for any $0<p<\infty$

$$
\mathbb{E} \sup _{t \geq 0}\left\|\left(\Phi \cdot W_{H}\right)_{t}\right\|^{p} \bar{\sim}_{p} \mathbb{E}\left(\int_{0}^{t}\|\Phi\|^{2} \mathrm{~d} s\right)^{p / 2} .
$$

We refer the reader to $[27,32,52,56,74,75,76,81,108,119]$ for further details on stochastic integration and cylindrical Brownian motions.

\subsection{1. $\gamma$-radonifying operators}

Let $H$ be a separable Hilbert space and let $X$ be a Banach space. Let $T \in$ $\mathcal{L}(H, X)$. Then $T$ is called $\gamma$-radonifying if

$$
\|T\|_{\gamma(H, X)}:=\left(\mathbb{E}\left\|\sum_{n=1}^{\infty} \gamma_{n} T h_{n}\right\|^{2}\right)^{\frac{1}{2}}<\infty
$$


where $\left(h_{n}\right)_{n \geq 1}$ is an orthonormal basis of $H$, and $\left(\gamma_{n}\right)_{n \geq 1}$ is a sequence of independent standard Gaussian random variables (if the series on the righthand side of (2.19) does not converge, then we set $\left.\|T\|_{\gamma(H, X)}:=\infty\right)$. Note that $\|T\|_{\gamma(H, X)}$ does not depend on the choice of the orthonormal basis $\left(h_{n}\right)_{n \geq 1}$ (see [47, Section 9.2] and [80] for details). Often we will call $\|T\|_{\gamma(H, X)}$ the $\gamma$-norm of $T$. Note that if $X$ is a Hilbert space, then $\|T\|_{\gamma(H, X)}$ coincides with the Hilbert-Schmidt norm of $T$. $\gamma$-norms are exceptionally important in analysis as they are easily computable and enjoy a number of useful properties such as the ideal property, $\gamma$-multiplier theorems, Fubini-type theorems, etc., see [47, 80].

\subsection{Tangent martingales: the discrete case}

Let $X$ be a Banach space, $\left(d_{n}\right)_{n \geq 1}$ and $\left(e_{n}\right)_{n \geq 1}$ be $X$-valued martingale difference sequences.

Definition 2.27. $\left(d_{n}\right)_{n \geq 1}$ and $\left(e_{n}\right)_{n \geq 1}$ are called tangent if

$$
\mathbb{P}\left(d_{n} \mid \mathcal{F}_{n-1}\right)=\mathbb{P}\left(e_{n} \mid \mathcal{F}_{n-1}\right), \quad n \geq 1 .
$$

(Recall that conditional probabilities have been defined in Subsection 2.2.)

Example 2.28. Let $\left(v_{n}\right)_{n \geq 1}$ be a predictable uniformly bounded $X$-valued sequence, $\left(\xi_{n}\right)_{n \geq 1}$ and $\left(\xi_{n}^{\prime}\right)_{n \geq 1}$ be adapted sequences of mean-zero real-valued independent random variables such that $\xi_{n}$ and $\xi_{n}^{\prime}$ are equidistributed, integrable, and independent of $\mathcal{F}_{n-1}$ for any $n \geq 1$. Then martingale difference sequences $\left(\xi_{n} v_{n}\right)_{n \geq 1}$ and $\left(\xi_{n}^{\prime} v_{n}\right)_{n \geq 1}$ are tangent. Indeed, for any $n \geq 1$ and $A \in \mathcal{B}(X)$ we have that a.s.

$$
\begin{aligned}
& \mathbb{P}\left(\xi_{n} v_{n} \mid \mathcal{F}_{n-1}\right)(A)=\mathbb{E}\left(\mathbf{1}_{A}\left(\xi_{n} v_{n}\right)(A) \mid \mathcal{F}_{n-1}\right)=\mathbb{E}\left(\mathbf{1}_{A / v_{n}}\left(\xi_{n}\right)(A) \mid \mathcal{F}_{n-1}\right) \\
& \quad \stackrel{(*)}{=} \mathbb{E}\left(\mathbf{1}_{A / v_{n}}\left(\xi_{n}^{\prime}\right)(A) \mid \mathcal{F}_{n-1}\right)=\mathbb{E}\left(\mathbf{1}_{A}\left(\xi_{n}^{\prime} v_{n}\right)(A) \mid \mathcal{F}_{n-1}\right)=\mathbb{P}\left(\xi_{n}^{\prime} v_{n} \mid \mathcal{F}_{n-1}\right)(A),
\end{aligned}
$$

where $(*)$ follows from the fact that $\xi_{n}$ and $\xi_{n}^{\prime}$ are i.i.d. and independent from $\mathcal{F}_{n-1}$, and the fact that $v_{n}$ is $\mathcal{F}_{n-1}$ measurable, where for $A \subset X$ and $x \in X$ we define $A / x \subset \mathbb{R}$ by

$$
A / x:=\{t \in \mathbb{R}: t x \in A\} .
$$

It was shown by Hitczenko in [44] (see also [24, 28, 29, 32, 46, 64]) that any $X$-valued martingale difference sequence $\left(d_{n}\right)_{n \geq 1}$ has a decoupled tangent martingale difference sequence on an enlarged probability space with an enlarged filtration, i.e. there exists an enlarged filtration $\overline{\mathbb{F}}$ w.r.t. which $\left(d_{n}\right)$ remains being a martingale difference sequence, an $\overline{\mathbb{F}}$-adapted martingale difference sequence $\left(e_{n}\right)_{n \geq 1}$, and a $\sigma$-algebra $\mathcal{G} \subset \mathcal{F}_{\infty}$ such that

$$
\mathbb{P}\left(e_{n} \mid \mathcal{F}_{n-1}\right)=\mathbb{P}\left(e_{n} \mid \mathcal{G}\right), \quad n \geq 1,
$$

and $\left(e_{n}\right)_{n \geq 1}$ are conditionally independent given $\mathcal{G}$ (see Subsection 2.2). Moreover, $\left(e_{n}\right)_{n \geq 1}$ is unique up to probability. Later in Section 3 we will extend a construction of such a martingale to the continuous-time case. 
Remark 2.29. Note that due to Proposition 2.6, the construction of a decoupled tangent martingale difference sequence [28, 29, 44], and the uniqueness of its distribution we can give the following equivalent definition: $\left(e_{n}\right)_{n>1}$ is a decoupled tangent martingale difference sequence to $\left(d_{n}\right)_{n \geq 1}$ if and only if for a.e. $\omega \in \Omega$ the sequence $\left(e_{n}(\omega)\right)_{n \geq 1}$ is a sequence of mean zero random variables so that $c_{n}(\omega)$ has $\mathbb{P}\left(d_{n} \mid \mathcal{F}_{n-1}\right)(\omega)$ as its distribution (see [28, 29] or the proof of Theorem 3.39 for the construction of $\left.\mathbb{P}\left(d_{n} \mid \mathcal{F}_{n-1}\right)(\omega)\right)$.

\section{Tangent martingales: the continuous-time case}

This section is devoted to continuous-time tangent martingales and their properties. As the notion of tangency in the continuous-times case (see Definition 3.1 below) only cares about the jumps of a process and the quadratic variation of its continuous part, throughout this section we will assume that any martingale starts in zero. Also, in the sequel we will frequently use the stopping times argument which is allowed by Theorem A.3. In particular, while talking about tangent local martingales $M$ and $N$ we can automatically assume that these martingales have finite strong $L^{1}$-moment, i.e. $\mathbb{E} \sup _{t>0}\left\|M_{t}\right\|$ and $\mathbb{E} \sup _{t>0}\left\|N_{t}\right\|$ can be presumed to be finite unless stated otherwise (see Remark 2.10).

\subsection{Local characteristics and tangency}

In order to define tangent martingales in the continuous-time case we need local characteristics.

Let $M: \mathbb{R}_{+} \times \Omega \rightarrow \mathbb{R}$ be a local martingale, $M=M^{c}+M^{d}$ be the MeyerYoeurp decomposition of $M$ (see Remark 2.19). Then the pair $\left(\left[M^{c}\right], \nu^{M}\right)$, where $\left[M^{c}\right]$ is the quadratic variation of $M^{c}$ (see Subsection 2.6), and $\nu^{M}$ is a compensator of the random measure $\mu^{M}$ defined by (2.14), is called the local characteristics of $M$.

Let $X$ be a Banach space, $M$ be an $X$-valued local martingale. Assume that $M$ admits the Meyer-Yoeurp decomposition $M=M^{c}+M^{d}$ (see Remark 2.19) and that $M^{c}$ has a covariation bilinear form $\llbracket M^{c} \rrbracket$ (see Subsection 2.6). Then we define the local characteristics of $M$ to be the pair $\left(\llbracket M^{c} \rrbracket, \nu^{M}\right)$, where $\nu^{M}$ is a compensator of the random measure $\mu^{M}$ defined by (2.14).

Definition 3.1. Two $X$-valued local martingales $M$ and $N$ are called tangent if both local martingales have local characteristics which coincide.

Remark 3.2. Note that this definition of tangency agrees with the one for discrete martingales given in Subsection 2.12. Indeed, let $\left(d_{n}\right)_{n \geq 1}$ and $\left(e_{n}\right)_{n \geq 1}$ be tangent martingale difference sequences. Then they are tangent in the continuous-time case if for any $n \geq 1$ compensators of random measures $\mu^{d_{n}}$ and $\mu^{e_{n}}$ defined on $X$ by

$$
\mu^{d_{n}}(A)=\mathbf{1}_{A}\left(d_{n}\right), \quad \mu^{e_{n}}(A)=\mathbf{1}_{A}\left(e_{n}\right), \quad A \in \mathcal{B}(X),
$$


coincide. But these compensators exactly coincide with $\mathbb{P}\left(d_{n} \mid \mathcal{F}_{n-1}\right)$ and with $\mathbb{P}\left(e_{n} \mid \mathcal{F}_{n-1}\right)$ respectively as by the definition $(2.3)$ of $\mathbb{P}\left(d_{n} \mid \mathcal{F}_{n-1}\right)$ and $\mathbb{P}\left(e_{n} \mid \mathcal{F}_{n-1}\right)$ and by (2.20) for any Borel set $A \subset X$ one has that

$$
\begin{array}{r}
\mathbb{E}\left(\mu^{d_{n}}(A) \mid \mathcal{F}_{n-1}\right)=\mathbb{E}\left(\mathbf{1}_{A}\left(d_{n}\right) \mid \mathcal{F}_{n-1}\right)=\mathbb{P}\left(d_{n} \mid \mathcal{F}_{n-1}\right)(A) \\
=\mathbb{P}\left(e_{n} \mid \mathcal{F}_{n-1}\right)(A)=\mathbb{E}\left(\mathbf{1}_{A}\left(e_{n}\right) \mid \mathcal{F}_{n-1}\right)=\mathbb{E}\left(\mu^{e_{n}}(A) \mid \mathcal{F}_{n-1}\right) .
\end{array}
$$

The converse direction can be shown similarly.

Now we are ready to define a decoupled tangent local martingale. Recall that conditional independence was defined in (2.4) and an enlargement of a filtered probability space was defined in Definition 2.3.

Definition 3.3. Let $(\Omega, \mathcal{F}, \mathbb{P})$ be a probability space endowed with a filtration $\mathbb{F}=\left(\mathcal{F}_{t}\right)_{t \geq 0}$. Let $M: \mathbb{R}_{+} \times \Omega \rightarrow X$ be a local martingale. A process $N$ : $\mathbb{R}_{+} \times \bar{\Omega} \rightarrow X$ over an enlarged probability space $(\bar{\Omega}, \overline{\mathcal{F}}, \overline{\mathbb{P}})$ with an enlarged filtration $\overline{\mathbb{F}}=\left(\overline{\mathcal{F}}_{t}\right)_{t \geq 0}$ is called a decoupled tangent local martingale of $M$ if $M$ is a local $\overline{\mathbb{F}}$-martingale with the same local characteristics $\left(\llbracket M^{c} \rrbracket, \nu^{M}\right), N$ is a local martingale, $M$ and $N$ are tangent, and $N(\omega)$ is a local martingale with independent increments and local characteristics $\left(\llbracket M^{c} \rrbracket(\omega), \nu^{M}(\omega)\right)$ for a.e. $\omega \in \Omega$.

Note that we can always set $\mathcal{F}:=\mathcal{F}_{\infty}$, and that $N$ may be assumed to have independent increments given $\mathcal{F}$ due to Proposition 2.6. We refer the reader to $[64$, p. 174] and $[24,28,29,51,58,63,65]$ for further details on decoupled tangent local martingales.

Remark 3.4. Note that the martingale properties of $M$ in Definition 3.3 could change while enlarging the filtration, so it is extremely important to presume that $M$ is a local $\overline{\mathbb{F}}$-martingale having the same local characteristics as it used to obtain. Indeed, first $M$ can lose its martingality, e.g. if $\overline{\mathcal{F}}_{t}$ contains $\mathcal{F}_{\infty}$ for any $t \geq 0$ (then $M_{t}$ is $\overline{\mathcal{F}}_{0}$-measurable). But even if $M$ remains a local martingale, it could change its local characteristics. Though $\llbracket M^{c} \rrbracket$ would stay the same for any filtration (due to the fact that $\llbracket M^{c} \rrbracket$ is a continuous part of $\llbracket M \rrbracket$, see Subsection 2.7, [56, Theorem 26.14], and [116], and the fact that $\llbracket M \rrbracket$ does not depend on the enlargement by its definition (2.7)), $\nu^{M}$ can change. E.g. let $X=\mathbb{R}$ and let $M=N^{1}-N^{2}$, where $N^{1}$ and $N^{2}$ are independent standard Poisson processes. Let $\mathbb{F}=\left(\mathcal{F}_{t}\right)_{t \geq 0}$ be generated by $M$ and let $\overline{\mathcal{F}}_{t}$ be generated by $\mathcal{F}_{t}$ and $\sigma\left(\left(\tau_{n}\right)_{n \geq 1}\right)$, where $\left(\tau_{n}\right)_{n \geq 1}$ are all jump times of $M$. Then $M$ is both an $\mathbb{F}$ - and $\overline{\mathbb{F}}$-martingale, but for any $A \subset \mathbb{R}$ and $I \subset \mathbb{R}_{+}$in the first case we have that $\nu^{M}(I \times A)=\lambda_{\mathbb{R}_{+}}(I)\left(\mathbf{1}_{A}(-1)+\mathbf{1}_{A}(1)\right)$, but in the second case we obtain $\nu^{M}(I \times A)=\frac{1}{2} \sum_{n \geq 1} \mathbf{1}_{I}\left(\tau_{n}\right)\left(\mathbf{1}_{A}(-1)+\mathbf{1}_{A}(1)\right)$.

Remark 3.5. Note that every local martingale with independent increments is a martingale by [29, Theorem 2.5.1]. Indeed, let $M: \mathbb{R}_{+} \times \Omega \rightarrow X$ be a local martingale with independent increments. Then there exists a sequence $\left(\tau_{n}\right)_{n \geq 1}$ of stopping times such that $\tau_{n} \nearrow \infty$ a.s. as $n \rightarrow \infty$ and $M^{\tau_{n}}$ is a martingale. Moreover, by strengthening stopping times $\left(\tau_{n}\right)_{n \geq 1}$ we can assume that 
$\mathbb{E} \sup _{t \geq 0}\left\|M_{t}^{\tau_{n}}\right\|<\infty$. Then by [29, Theorem 2.5.1] we have that for any $n \geq 1$

$$
\mathbb{E} \sup _{t \geq 0}\left\|M_{t}^{\tau_{n}}\right\| \approx \mathbb{E} \sup _{t \geq 0}\left\|\widetilde{M}_{t}^{\tau_{n}}\right\|<\infty
$$

where $\widetilde{M}$ is an independent copy of $M . A s \tau_{n}$ and $\widetilde{M}$ are independent, we have that

$$
\mathbb{E} \sup _{0 \leq s \leq t}\left\|\widetilde{M}_{s}\right\|=\mathbb{E} \sup _{0 \leq s \leq t}\left\|M_{s}\right\|<\infty
$$

for any $t \geq 0$ safistying $\mathbb{P}\left(\tau_{n}>t\right)>0$. Since $\tau_{n} \rightarrow \infty$ a.s. as $n \rightarrow \infty$, (3.2) holds for any $t \geq 0$, and thus $M$ is a martingale by a standard argument. Consequently, $N(\omega)$ in the definition above is can be assume to be a martingale instead of a local martingale.

Remark 3.6. Let us show that this definition of a decoupled tangent martingale agrees with the one given Subsection 2.12, i.e. if we have two martingales $M, N: \mathbb{R}_{+} \times \Omega \rightarrow X$ which are purely discontinuous and have jumps only at natural points, then $N s$ a decoupled tangent martingale to $M$ if and only if the same holds for the corresponding differences. Let $\left(d_{n}\right)_{n \geq 1}$ be an $X$-valued martingale difference sequence, $\left(c_{n}\right)_{n \geq 1}$ be a decoupled tangent martingale difference sequence. Let $M$ and $N$ be martingales with respect to the filtration $\mathbb{F}=\left(\mathcal{F}_{t}\right)_{t \geq 0}:=\left(\mathcal{F}_{[t]}\right)_{t \geq 0}$ (here $\left(\mathcal{F}_{n}\right)_{n \geq 1}$ is the filtration where $\left(d_{n}\right)_{n \geq 1}$ lives and $[a]$ is the integer part of $a \geq 0)$ of the form

$$
M_{t}=\sum_{n \leq t} d_{n}, \quad N_{t}=\sum_{n \leq t} c_{n}, \quad t \geq 0 .
$$

Then $M$ and $N$ are tangent by (3.1), and $N(\omega)$ is a martingale with independent increments and local characteristics $\left(0, \nu^{M}(\omega)\right)$ as the same holds for $\left(c_{n}\right)_{n \geq 1}$ thanks to Remark 2.29, so $N$ is a decoupled tangent martingale to $M$. The converse can be shown analogously.

Now we are going to state two main results of the paper.

Theorem 3.7. Let $X$ be a Banach space. Then $X$ is UMD if and only if any $X$ valued local martingale has local characteristic. Moreover, if this is the case, then for any tangent $X$-valued local martingales $M$ and $N$ and for any $1 \leq p<\infty$ we have that

$$
\mathbb{E} \sup _{t \geq 0}\left\|M_{t}\right\|^{p} \bar{\sim}_{p, X} \mathbb{E} \sup _{t \geq 0}\left\|N_{t}\right\|^{p} .
$$

Theorem 3.8. Let $X$ be a UMD Banach space. Then for any $X$-valued local martingale there exists a decoupled tangent local martingale.

In order to prove these theorems we will need to treat each of the cases of the canonical decompositions separately in Subsection 3.3, 3.5, 3.6, and then combine them using Subsection 3.2 in Subsection 3.7. 


\subsection{Local characteristics and canonical decomposition}

Let us first show that different parts of the canonical decomposition are responsible for different parts of the corresponding local characteristics, and in particular that if two martingales are tangent, then the same holds for the corresponding parts of the canonical decomposition.

Theorem 3.9. Let $X$ be a Banach space, $M, N: \mathbb{R}_{+} \times \Omega \rightarrow X$ be local martingales that have the canonical decompositions $M=M^{c}+M^{q}+M^{a}$ and $N=N^{c}+N^{q}+N^{a}$. Assume also that both $M$ and $N$ have local characteristics $\left(\llbracket M^{c} \rrbracket, \nu^{M}\right)$ and $\left(\llbracket N^{c} \rrbracket, \nu^{N}\right)$ respectively, and that $M$ and $N$ are tangent. Then the corresponding terms of the canonical decomposition have local characteristics and are tangent as well.

We will prove the theorem by using the following elementary propositions.

Proposition 3.10. Let $X$ be a Banach space, $M: \mathbb{R}_{+} \times \Omega \rightarrow X$ be a continuous local martingale which has a covariation bilinear form $\llbracket M \rrbracket$. Then the local characteristics of $M$ are $(\llbracket M \rrbracket, 0)$.

Proof. As $M$ does not have jumps, $M=M+0$ is the Meyer-Yoeurp decomposition; moreover, $\mu^{M}=0$ a.s., and hence $\nu^{M}=0$ a.s.

Proposition 3.11. Let $X$ be a Banach space, $M: \mathbb{R}_{+} \times \Omega \rightarrow X$ be a purely discontinuous quasi-left continuous local martingale. Then the local characteristics of $M$ are $\left(0, \nu^{M}\right)$, where $\nu^{M}$ is non-atomic in time.

Proof. First notice that $M$ is purely discontinuous, hence $M^{c}=0$, and thus $\llbracket M^{c} \rrbracket=0$. The fact that $\nu^{M}$ is non-atomic in time follows from Lemma 2.22.

Proposition 3.12. Let $X$ be a Banach space, $M: \mathbb{R}_{+} \times \Omega \rightarrow X$ be a purely discontinuous local martingale with accessible jumps. Then the local characteristics of $M$ are $\left(0, \nu^{M}\right)$, where for $\nu^{M}$ there exist predictable stopping times $\left(\tau_{n}\right)_{n \geq 1}$ such that for any $A \in \widetilde{\mathcal{P}}$ we have that a.s.

$$
\int_{\mathbb{R}_{+} \times X} \mathbf{1}_{A}(t, \cdot, x) \mathrm{d} \nu^{M}(t, x)=\int_{\mathbb{R}_{+} \times X} \mathbf{1}_{A}(t, \cdot, x) \mathbf{1}_{\left\{\tau_{1}, \ldots, \tau_{n}, \ldots\right\}}(t) \mathrm{d} \nu^{M}(t, x) .
$$

In other words, $\left\{\tau_{1}, \ldots, \tau_{n}, \ldots\right\} \times X$ is a set of full $\nu^{M}$-measure a.s.

For the proof we will need the following lemma, which follows from [32, Subsection 5.3].

Lemma 3.13. Let $(J, \mathcal{J})$ be a measurable space, $\mu$ be an integer-valued random measure on $\mathbb{R}_{+} \times \Omega$, and let $\tau$ be a predictable stopping times such that $\mu \mathbf{1}_{\tau}=\mu$. Then for the corresponding compensator $\nu$ we have we have that $\nu \mathbf{1}_{\tau}=\nu$, i.e. $\{\tau\} \times X$ is a set of full $\nu^{M}$-measure a.s.

Proof of Proposition 3.12. First notice that $\llbracket M^{c} \rrbracket=0$ analogously to Proposition 3.11. As $M$ is purely discontinuous with accessible jumps, by Lemma 2.20 
there exists a sequence $\left(\tau_{n}\right)_{n \geq 1}$ of finite predictable stopping times with disjoint graphs such that a.s.

$$
\left\{t \geq 0: \Delta M_{t} \neq 0\right\} \subset\left\{\tau_{1}, \ldots, \tau_{n}, \ldots\right\} .
$$

Then the desired follows from the fact that $\mu^{M}=\sum_{n \geq 1} \mu^{M} \mathbf{1}_{\tau_{n}}$ a.s., Lemma 3.13, and the fact that $\nu^{M}=\sum_{n \geq 1} \nu^{M} \mathbf{1}_{\tau_{n}}$, which follows e.g. from [52, Chapter II.1]. Indeed, $\mu^{M}$ is $\widetilde{\mathcal{P}}$ - $\sigma$-finite, and if we fix $A \in \widetilde{\mathcal{P}}$ such that $\mathbb{E} \int_{\mathbb{R}_{+} \times X} \mathbf{1}_{A} \mathrm{~d} \mu^{M}<\infty$, then by the monotone convergence theorem and by the definition of a compensator measure (see Subsection 2.8 and [52, Chapter II.1])

$$
\begin{aligned}
\mathbb{E} \int_{\mathbb{R}_{+} \times X} \mathbf{1}_{A} \mathrm{~d} \nu^{M}=\mathbb{E} \int_{\mathbb{R}_{+} \times X} \mathbf{1}_{A} \mathrm{~d} \mu^{M}=\sum_{n \geq 1} \mathbb{E} \int_{\mathbb{R}_{+} \times X} \mathbf{1}_{A} \mathbf{1}_{\tau_{n}} \mathrm{~d} \mu^{M} \\
=\mathbb{E} \int_{\mathbb{R}_{+} \times X} \mathbf{1}_{A} \mathbf{1}_{\left\{\tau_{1}, \ldots, \tau_{n} \ldots\right\}} \mathrm{d} \mu^{M}=\mathbb{E} \int_{\mathbb{R}_{+} \times X} \mathbf{1}_{A} \mathbf{1}_{\left\{\tau_{1}, \ldots, \tau_{n} \ldots\right\}} \mathrm{d} \nu^{M},
\end{aligned}
$$

so (3.4) follows immideately.

Proposition 3.14. Let $X$ be a Banach space, $M: \mathbb{R}_{+} \times \Omega \rightarrow X$ be a local martingale. Assume that $M$ admits the canonical decomposition $M=M^{c}+$ $M^{q}+M^{a}$. Then we have that $\nu^{M}=\nu^{M^{q}}+\nu^{M^{a}}$.

Proof. Note that by Definition 2.15 of the canonical decomposition and by Remark 2.17, $M^{q}$ and $M^{a}$ collect different jumps of $M$, i.e. (2.8) holds true, and hence $\mu^{M^{q}}+\mu^{M^{a}}=\mu^{M}$, so $\nu^{M^{q}}+\nu^{M^{a}}=\nu^{M}$ by the definition of a compensator (see Subsection 2.8).

We will also need the following lemma, which follows from [57, Theorem 9.22]. Recall that a random measure $\mu$ with a compensator $\nu$ is called quasi-left continuous if any stochastic integral with respect to $\bar{\mu}=\mu-\nu$ defined by (2.12) is quasi-left continuous. A random measure $\mu$ with a compensator $\nu$ is called accessible if any stochastic integral with respect to $\bar{\mu}$ has accessible jumps.

Lemma 3.15. Let $(J, \mathcal{J})$ be a measurable space, $\mu$ be an integer-valued $\widetilde{P}-\sigma$ finite random measure on $\mathbb{R}_{+} \times \Omega$. Then there exist unique random measures $\mu^{q}$ and $\mu^{a}$ such that $\mu=\mu^{q}+\mu^{a}$ and such that $\mu^{q}$ is quasi-left continuous and $\mu^{a}$ is accessible.

Remark 3.16. Note that analogously to Lemma 2.22 one can show that $\mu$ is quasi-left continuous if and only if $\nu$ is non-atomic in time. Similarly, by the fact that any accessible measure is supported by countably many predictable stopping times (see [57, Theorem 9.22]) and by applying techniques from the proof of Proposition 3.12 it follows that $\mu$ is accessible if and only if its compensator $\nu$ has a.s. a support of countably many points on $\mathbb{R}_{+}$.

Proof of Theorem 3.9. The theorem follows from Proposition 3.10, 3.11, 3.12, and 3.14, Lemma 3.15, and Remark 3.16. 


\subsection{Continuous martingales}

First let us consider continuous martingales. The theory of continuous martingales is already classical and in particular due to [36, 81, 119] the following proposition holds true.

Proposition 3.17. Let $X$ be a Banach space, $0<p<\infty$. Then $X$ is UMD if and only if for any pair $M, N: \mathbb{R}_{+} \times \Omega \rightarrow X$ of continuous martingales with $\llbracket N \rrbracket_{\infty} \leq \llbracket M \rrbracket_{\infty}$ a.s. one has that

$$
\mathbb{E} \sup _{1 \leq t<\infty}\left\|N_{t}\right\|^{p} \lesssim_{p, X} \mathbb{E} \sup _{1 \leq t<\infty}\left\|M_{t}\right\|^{p}
$$

What we are interested in is constructing for an $X$-valued continuous martingale $M$ a decoupled tangent martingale $N$ (see Definition 3.3), which we will need later in Subsection 3.7.

Theorem 3.18. Let $X$ be a UMD Banach space, $M: \mathbb{R}_{+} \times \Omega \rightarrow X$ be a continuous local martingale. Then there exists an enlarged probability space $(\bar{\Omega}, \overline{\mathcal{F}}, \overline{\mathbb{P}})$ endowed with an enlarged filtration $\overline{\mathbb{F}}=\left(\overline{\mathcal{F}}_{t}\right)_{t \geq 0}$, and an $\overline{\mathbb{F}}$-adapted continuous local martingale $N: \mathbb{R}_{+} \times \bar{\Omega} \rightarrow X$ such that $M$ is a local $\overline{\mathbb{F}}$-martingale with the same local characteristics, $M$ and $N$ are tangent, and $N(\omega)$ is a martingale with independent increments and with local characteristics $(\llbracket M \rrbracket(\omega), 0)$ for a.e. $\omega \in \Omega$.

For the proof we will need the following statement concerning Brownian representations.

Lemma 3.19 (Brownian representation). Let $\left(M^{n}\right)_{n \geq 1}$ be a family of continuous martingales and $\left(a_{n}\right)_{n \geq 1}$ be a real-valued sequence such that a.s. for any $n \geq 1$ and any $0 \leq s \leq t$

$$
\left[M^{n}\right]_{t}-\left[M^{n}\right]_{s} \leq a_{n}(t-s) .
$$

Then there exists a Hilbert space $H$, an enlarged probability space $(\bar{\Omega}, \overline{\mathcal{F}}, \overline{\mathbb{P}})$ endowed by an enlarged filtration $\overline{\mathbb{F}}=\left(\overline{\mathcal{F}}_{t}\right)_{t \geq 0}$, an $\overline{\mathbb{F}}$-adapted cylindrical Brownian motion $W_{H}$, and a family of predictable $H$-valued processes $\left(f_{n}\right)_{n \geq 1}$ depending only on the family of processes $\left(\left[M^{n}, M^{m}\right]\right)_{n, m \geq 1}$ such that

$$
M^{n}=f_{n} \cdot W_{H}, \quad n \geq 1 .
$$

Proof. Let $H$ be a separable Hilbert space, $\left(h_{n}\right)_{n \geq 1}$ be an orthonormal basis of $H$. Let us define an $H$-valued process $M:=\sum_{n \geq 1} \frac{1}{\sqrt{a_{n}} n} M^{n} h_{n}$. First let us show that this process is well defined. To this end we need to show that $\sum_{n=1}^{\infty}\left|\frac{1}{\sqrt{a_{n}} n} M_{t}^{n}\right|^{2}$ converges a.s. for any $t \geq 0$. Note that by the monotone convergence theorem one has that a.s.

$$
\begin{aligned}
\mathbb{E} \sum_{n=1}^{\infty}\left|\frac{1}{\sqrt{a_{n}} n} M_{t}^{n}\right|^{2}= & \lim _{N \rightarrow \infty} \mathbb{E} \sum_{n=1}^{N} \frac{1}{a_{n} n^{2}}\left|M_{t}^{n}\right|^{2} \\
& \stackrel{(*)}{=} \lim _{N \rightarrow \infty} \mathbb{E} \sum_{n=1}^{N} \frac{1}{a_{n} n^{2}}\left[M^{n}\right]_{t} \leq \mathbb{E} \sum_{n=1}^{\infty} \frac{1}{n^{2}} t \lesssim t,
\end{aligned}
$$


where $(*)$ holds by [56, Theorem 26.12]. Therefore $\sum_{n=1}^{\infty}\left|\frac{1}{\sqrt{a_{n}} n} M_{t}^{n}\right|^{2}$ converges in measure, and as this is a sum of nonnegative random variables, it converges a.s. For the similar reason and by the continuity of the conditional expectation [46, Section 2.6] one has that $M$ is an $H$-valued martingale so that by e.g. [108, (3.8)] for any $0 \leq s \leq t$ a.s.

$$
\begin{aligned}
{[M]_{t}-[M]_{s}=\sum_{n=1}^{\infty}\left[\left\langle M, h_{n}\right\rangle\right]_{t}-\left[\left\langle M, h_{n}\right\rangle\right]_{s} } & =\sum_{n=1}^{\infty} \frac{1}{a_{n} n^{2}}\left(\left[M^{n}\right]_{t}-\left[M^{n}\right]_{s}\right) \\
& \leq \sum_{n=1}^{\infty} \frac{1}{n^{2}}(t-s) \lesssim t-s .
\end{aligned}
$$

Consequently, by [108, Example 3.18 and Proposition 4.7] (see also [88, Theorem $2]$, [54, 114], [27, Theorem 8.2], and [60, Theorem 3.4.2]) there exist an enlarged probability space $(\bar{\Omega}, \overline{\mathcal{F}}, \overline{\mathbb{P}})$ endowed by an enlarged filtration $\overline{\mathbb{F}}=\left(\overline{\mathcal{F}}_{t}\right)_{t \geq 0}$, an $\overline{\mathbb{F}}$ adapted cylindrical Brownian motion $W_{H}$, and a predictable process $\bar{\Phi}: \mathbb{R}_{+} \times$ $\Omega \rightarrow \mathcal{L}(H)$ such that $M=\Phi \cdot W_{H}$. Notice that by the construction of $\Phi$ presented in [108, Proposition 4.7] it depends only on the family of processes $([\langle M, h\rangle,\langle M, g\rangle])_{h, g \in H}$, and the latter by the fact that covariation is bilinear depends only on the family of processes

$$
\left(\left[\left\langle M, h_{n}\right\rangle,\left\langle M, h_{m}\right\rangle\right]\right)_{n, m \geq 1}=\left(\left[M^{n}, M^{m}\right]\right)_{n, m \geq 1} .
$$

The desired follows by setting $f_{n}:=\sqrt{a_{n}} n \Phi^{*} h_{n}$ for any $n \geq 1$, so

$$
f_{n} \cdot W_{H}=\sqrt{a_{n}} n \Phi^{*} h_{n} \cdot W_{H}=\sqrt{a_{n}} n\left\langle\Phi \cdot W_{H}, h_{n}\right\rangle=\sqrt{a_{n}} n\left\langle M, h_{n}\right\rangle=M^{n} .
$$

Proof of Theorem 3.18. Without loss of generality by the Pettis measurability theorem [46, Theorem 1.1.20] we may assume that $X$ is separable (and as $X$ is $\mathrm{UMD}$, it is reflexive, so $X^{*}$ is separable as well). By a stopping time argument we may assume that $M$ is uniformly bounded a.e. on $\mathbb{R}_{+} \times \Omega$. Let $\left(x_{n}^{*}\right)_{n \geq 1}$ be a dense subset of a unit ball in $X^{*}$, and let us define a random time-change

$$
\tau_{t}:=\inf \left\{s \geq 0: \sum_{n=1}^{\infty} \frac{1}{n^{2}}\left[\left\langle M, x_{n}^{*}\right\rangle\right]_{s}+s \geq t\right\}, \quad t \geq 0
$$

(the latter time-change is well defined as $\left(\left[\left\langle M, x_{n}^{*}\right\rangle\right]\right)_{n \geq 1}$ are a.s. uniformly bounded since by [119]

$$
\left[\left\langle M, x^{*}\right\rangle\right]_{s} \leq\left\|\llbracket M \rrbracket_{s}\right\| \leq \gamma\left(\llbracket M \rrbracket_{s}\right)<\infty, \quad s \geq 0,
$$

a.s. for any $x^{*} \in X^{*},\left\|x^{*}\right\| \leq 1$, see Remark 2.13 for the definition of $\left.\gamma(\cdot)\right)$. Note that as stricktly increasing, this time-change is invertible, i.e. for a time changed filtration $\mathbb{G}=\left(\mathcal{G}_{t}\right)_{t \geq 0}:=\left(\mathcal{F}_{\tau_{t}}\right)_{t \geq 0}$ there exists a strictly increasing $\mathbb{F}$-predictable continuous process $A: \mathbb{R}_{+} \times \Omega \rightarrow X$ such that $(A \circ \tau)_{t}=(\tau \circ A)_{t}=t$ a.s. for any $t \geq 0$. (Note that in fact $A_{t}=\sum_{n=1}^{\infty} \frac{1}{n^{2}}\left[\left\langle M, x_{n}^{*}\right\rangle\right]_{t}+t$ as it is defined by (3.6), 
see e.g. [116, Theorem 2.16] or [117, Subsection 2.4.4]). Let $\widetilde{M}:=M \circ \tau$. Then by the Kazamaki theorem [56, Theorem 17.24] for any $n \geq 1$ a.s.

$$
\begin{aligned}
{\left[\left\langle\widetilde{M}, x_{n}^{*}\right\rangle\right]_{t}-\left[\left\langle\widetilde{M}, x_{n}^{*}\right\rangle\right]_{s} } & =\left[\left\langle M, x_{n}^{*}\right\rangle\right]_{\tau_{t}}-\left[\left\langle M, x_{n}^{*}\right\rangle\right]_{\tau_{s}} \\
& \leq n^{2}(t-s), \quad 0 \leq s \leq t,
\end{aligned}
$$

thus by Lemma 3.19 we can show that there exist an enlarged probability space $(\bar{\Omega}, \overline{\mathcal{F}}, \overline{\mathbb{P}})$, an enlarged filtration $\overline{\mathbb{G}}=\left(\overline{\mathcal{G}}_{t}\right)_{t \geq 0}$, a Hilbert space $H$, a $\overline{\mathbb{G}}$-adapted cylindrical Wiener process $W_{H}$, and a family of $H$-valued $\overline{\mathbb{G}}$-predictable processes $\left(f_{n}\right)$ such that

$$
\left\langle\widetilde{M}_{t}, x_{n}^{*}\right\rangle=\int_{0}^{t} f_{n} \mathrm{~d} W_{H}, \quad n \geq 1 .
$$

Let us first show that for any $x^{*} \in X^{*}$ there exists a $\overline{\mathbb{G}}$-predictable process $f_{x^{*}}: \mathbb{R}_{+} \times \Omega \rightarrow H$ such that $\left\langle\widetilde{M}_{t}, x^{*}\right\rangle=f_{x^{*}} \cdot W_{H}$. Let $Y=\operatorname{span}\left\{x_{n}^{*}, n \geq 1\right\} \subset$ $X^{*}$. By the definition of $\left(x_{n}^{*}\right)_{n \geq 1}, Y$ is dense in $X^{*}$, so there exists a sequence $\left(y_{m}\right)_{m \geq 1} \in Y$ that converges to $x^{*}$. By the definition of $Y$, by the linearity of a stochastic integral, and by (3.7) for each $m \geq 1$ there exists a $\overline{\mathbb{G}}$-predictable process $f_{y_{m}}: \mathbb{R}_{+} \times \Omega \rightarrow H$ such that $\left\langle\widetilde{M}_{t}, y_{m}\right\rangle=f_{y_{m}} \cdot W_{H}$. Moreover, $f_{y_{m}} \cdot W_{H}$ converges to $\left\langle\widetilde{M}, x^{*}\right\rangle$ in strong $L^{p}$ sense for any $1 \leq p<\infty$ as

$$
\begin{aligned}
\mathbb{E} \sup _{t \geq 0}\left|\int_{0}^{t} f_{y_{m}} \mathrm{~d} W_{H}-\left\langle\widetilde{M}_{t}, x^{*}\right\rangle\right|^{p} & =\mathbb{E} \sup _{t \geq 0}\left|\left\langle\widetilde{M}, x^{*}-y_{m}\right\rangle\right|^{p} \\
& \leq\left\|x^{*}-y_{m}\right\|^{p} \mathbb{E} \sup _{t \geq 0}\|\widetilde{M}\|^{p} \rightarrow 0, \quad m \rightarrow \infty,
\end{aligned}
$$

where the latter follows from the fact that $\widetilde{M}$ is uniformly bounded. Therefore the existence of the desired $f_{x^{*}}$ follows e.g. from (2.18) and the fact that the space $L^{p}\left(\bar{\Omega} ; L^{2}\left(\mathbb{R}_{+} ; H\right)\right)$ restricted to a proper predictable $\sigma$-algebra is a Banach space. Moreover, it follows from (2.17) that for any $x^{*} \in X^{*}$ for a.e. $\omega \in \bar{\Omega}$

$$
\int_{0}^{\infty}\left\|f_{x^{*}}\right\|^{2} \mathrm{~d} s=\left[\widetilde{M}, x^{*}\right]_{\infty}=\llbracket \widetilde{M} \rrbracket_{\infty}\left(x^{*}, x^{*}\right) \lesssim_{M, \omega}\left\|x^{*}\right\|^{2} .
$$

Let us now show that for a.e. $\omega \in \bar{\Omega}$ the mapping $x^{*} \mapsto f_{x^{*}}(\omega)$ can be assumed to be linear. As $Y$ is a linear subspace of $X^{*}$ generated by a countable set, it has a countable Hamel basis $\left(z_{n}\right)_{n \geq 1} \subset X^{*}$. Let $Z$ be a $\mathbb{Q}$-span of $\left(z_{n}\right)_{n \geq 1}$. Then by the linearity of a stochastic integral and by the fact that $Z$ is countable for any $z^{1}, \ldots, z^{k} \in Z$ and for any $r_{1}, \ldots, r_{k} \in \mathbb{Q}$ we can assume that $f_{r_{1} z^{1}+\ldots+r_{k} z^{k}}=$ $r_{1} f_{z^{1}}+\ldots+r_{k} f_{z^{k}}$ everywhere on $\bar{\Omega}$. Moreover, without loss of generality since $Z$ is countable we know that (3.8) holds a.s. for any $z \in Z$. Thus there exists $\bar{\Omega}_{0} \subset \bar{\Omega}$ of full measure such that for any $\omega \in \bar{\Omega}_{0}$ we have a bounded linear operator $T(\omega): Z \rightarrow L^{2}\left(\mathbb{R}_{+} ; H\right)$ with $T(\omega) z=f_{z}(\omega)$. By extending the operator $T(\omega)$ to the whole $X^{*}$ and by the construction of $f_{x^{*}}$ for a general $x^{*} \in X^{*}$ we have that $T(\omega) x^{*}=f_{x^{*}}(\omega)$ for a.e. $\omega \in \bar{\Omega}$, and the desired holds true. 
Now note that by $(3.8), T^{*}$ corresponds to a bilinear form $\llbracket M \rrbracket_{\infty}$ having a finite Gaussian characteristic (i.e. a.s. $\llbracket M \rrbracket_{\infty}\left(x^{*}, x^{*}\right)=\left\langle T x^{*}, T x^{*}\right\rangle$ for any $x^{*} \in X^{*}$ with $\gamma\left(\llbracket M \rrbracket_{\infty}\right)<\infty$, see [119, Subsection 3.2] and Remark 2.13), so by [119, Subsection 3.2] a.s. there exists $\Phi=T^{*} \in \gamma\left(L^{2}\left(\mathbb{R}_{+} ; H\right), X\right)$ such that a.s.

$$
\left\langle\widetilde{M}_{t}, x^{*}\right\rangle=\int_{0}^{t} f_{x^{*}} \mathrm{~d} W_{H}=\int_{0}^{t} \Phi^{*} x^{*} \mathrm{~d} W_{H}, \quad x^{*} \in X^{*},
$$

and thus by [81, Theorem 3.5] and the fact that $\Phi^{*} x^{*}=T x^{*}=f_{x^{*}}$ is a predictable process for any $x^{*} \in X^{*}$ we have that $\widetilde{M}=\Phi \cdot W_{H}$.

Finally, let us construct $N$. Let $W_{H}^{\prime}$ be an independent cylindrical Brownian motion, $\widetilde{N}:=\Phi \cdot W_{H}^{\prime}$, and let $N:=\widetilde{N} \circ A$. Then $N$ is a martingale on an enlarged probability space $(\widehat{\Omega}, \widehat{\mathcal{F}}, \widehat{\mathbb{P}})$ and an enlarged filtration $\widehat{\mathbb{F}}=\left(\widehat{\mathcal{F}}_{t}\right)_{t \geq 0}$ (which is now generated by the original filtration and time changed cylindrical Brownian motions $W_{H}$ and $W_{H}^{\prime}$ ) by Kazamaki theorem [56, Theorem 17.24] and the stochastic integration theory [81], so we need to show that $M$ is a local martingale with the same local characteristics $(\llbracket M \rrbracket, 0), \llbracket N \rrbracket=\llbracket M \rrbracket$ a.s., and that $N(\omega)$ is a martingale with independent increments and with the local characteristics $(\llbracket M \rrbracket(\omega), 0)$ for a.e. $\omega \in \Omega . M$ is a martingale because of the fact that $M$ is independent of $W_{H}^{\prime}$ and the fact that $M \circ \tau=\Phi \cdot W_{H}$ is a $\overline{\mathbb{G}}-$ martingale, so when we time-change it back by using $A$ we get a martingale with respect to the filtration generated by the original filtration $\mathbb{F}$ and a time-changed cylidrical process $W_{H}^{\prime} \circ A$ (see Kazamaki theorem [56, Theorem 17.24]), while $M$ does not change its local characteristics as it remains continuous and due to the definition of a quadratic variation which does not depend on enlargement of filtration (see Subsection 2.6). The second holds by (2.17) and due to the fact that for any $x^{*} \in X^{*}$ a.s.

$$
\llbracket N \rrbracket_{t}\left(x^{*}, x^{*}\right)=\llbracket \widetilde{N} \rrbracket_{A_{t}}\left(x^{*}, x^{*}\right)=\int_{0}^{A_{t}}\left\|\Phi^{*} x^{*}\right\|^{2} \mathrm{~d} s=\llbracket \widetilde{M} \rrbracket_{A_{t}}\left(x^{*}, x^{*}\right)=\llbracket M \rrbracket_{t}\left(x^{*}, x^{*}\right) .
$$

Now let us prove that $N(\omega)$ is a martingale with independent increments and with the local characteristics $(\llbracket M \rrbracket(\omega), 0)$. This directly follows from the construction of a stochastic integral (see Subsection 2.10 and [81]), the fact that $\Phi(\omega)$ is deterministic and is in $\gamma\left(L^{2}\left(\mathbb{R}_{+} ; H\right), X\right)$ for a.e. $\omega \in \Omega$, the fact that the time change $\left(\tau_{t}(\omega)\right)_{t \geq 0}$ is deterministic for a.e. $\omega \in \Omega$, and the fact that $W_{H}^{\prime}$ does not depend on $\omega \in \Omega$.

Remark 3.20. One can straighten the latter proposition in the following way: $N$ has independent increments given $\llbracket M^{c} \rrbracket$. Indeed, due to the construction of $\Phi$ (see Lemma 3.19 and its proof) and $\left(\tau_{t}\right)_{t \geq 0}$ we have that these random elements are $\sigma\left(\llbracket M^{c} \rrbracket\right)$-measurable, so the desired follows from Corollary 2.7 as for a.e. fixed $\llbracket M^{c} \rrbracket$ both $\Phi$ and $\left(\tau_{t}\right)_{t \geq 0}$ are fixed.

\subsection{Stochastic integrals with respect to random measures}

Before treating the case of purely discontinuous quasi-left continuous martingales in Subsection 3.5 we will need to prove a similar result for stochastic 
integrals with respect to random measures (see Subsection 2.8). This case will be done via Cox processes.

\subsubsection{Cox process}

Let $(J, \mathcal{J})$ be a measurable space, $\mu$ be an optional integer-valued random measure on $\mathbb{R}_{+} \times J$ with a compensator $\nu$ which is non-atomic in time (which is equivalent to the fact that $\mu$ is quasi-left continuous, see [57, Theorem 9.22] or [52, Corollary II.1.19]). Due to Cox [22] (see also [23, 52, 56, 57, 61]) it is known that there exists an enlarged probability space $(\bar{\Omega}, \overline{\mathcal{F}}, \overline{\mathbb{P}})=(\Omega \times \widehat{\Omega}, \mathcal{F} \otimes \widehat{\mathcal{F}}, \mathbb{P} \otimes \widehat{\mathbb{P}})$ (where $(\widehat{\Omega}, \widehat{\mathcal{F}}, \widehat{\mathbb{P}})$ is an independent probability space where the corresponding Poisson random measure lives, see Example 3.21), an enlarged filtration $\overline{\mathbb{F}}=\left(\overline{\mathcal{F}}_{t}\right)_{t \geq 0}$, and a unique up to distribution integer-valued random measure $\mu_{\text {Cox }}$ on $\mathbb{R}_{+} \times J$ optional with respect to $\overline{\mathbb{F}}$ having $\nu$ as a compensator so that $\mu_{\text {Cox }}$ is conditionally Poisson given $\mathcal{F}$, i.e. for any $C \in \widetilde{\mathcal{P}}$ (see Subsection 2.8 for the definition of $\widetilde{\mathcal{P}}$ ) with $\mathbb{E} \int_{\mathbb{R}_{+} \times J} \mathbf{1}_{C} \mathrm{~d} \nu<\infty$

- processes $\mu_{\text {Cox }}\left(\left([0, \cdot] \times A_{1}\right) \cap C\right)$ and $\mu_{\mathrm{Cox}}\left(\left([0, \cdot] \times A_{2}\right) \cap C\right)$ are conditionally independent given $\mathcal{F}$ for any disjoint $A_{1}, A_{2} \in \mathcal{J}$,

- the random measure $\mu_{\mathrm{Cox}}(\omega)$ is a Poisson random measure and for almost any fixed $\omega \in \Omega$ (see Subsection 2.9).

Such a random measure $\mu_{\text {Cox }}$ is called a Cox process directed by $\nu$.

Example 3.21. Let $J$ be finite, $J=\{1, \ldots, n\}, \mathcal{J}$ be a $\sigma$-algebra generated by all atoms of $J$. Let $\mu$ be a random measure on $\mathbb{R}_{+} \times J$ with a compensator $\nu$ such that $\nu([0, t] \times J)<\infty$ a.s. for any $t \geq 0$. Then the construction of the Cox process $\mu_{\text {Cox }}$ directed by $\nu$ has the following form. Let $N$ be a standard Poisson random measure on $\mathbb{R}_{+} \times J$, i.e. $\int \mathbf{1}_{\{0\}} \mathrm{d} N, \ldots, \int \mathbf{1}_{\{n\}} \mathrm{d} N$ are independent Poissons and

$$
\mathbb{E} \int_{[0, t] \times J} \mathbf{1}_{\{m\}} \mathrm{d} N=t, \quad 1 \leq m \leq n .
$$

Then the desired measure has the following form

$$
\mu_{\text {Cox }}([0, t] \times\{m\}):=N([0, \nu([0, t] \times\{m\})] \times\{m\}), \quad 1 \leq m \leq n .
$$

In the case of a general $\widetilde{P}-\sigma$-finite compensator $\nu$ the latter can be expressed as the sum $\nu=\sum_{k \geq 1} \nu^{k}$ of compensators $\left(\nu^{k}\right)_{k \geq 1}$ with disjoint domains in $\widetilde{\mathcal{P}}$, where each of $\nu^{k}$ satisfies $\nu^{k}\left(\mathbb{R}_{+} \times J\right)<\infty$ a.s., and then the Cox process $\mu_{\text {Cox }}$ will have the form $\mu_{\mathrm{Cox}}=\sum_{k \geq 1} \mu_{\mathrm{Cox}}^{k}$, where each of $\mu_{\mathrm{Cox}}^{k}$ is constructed analogously (3.9), but with using independent standard Poisson random measures $N^{k}$ on $\mathbb{R}_{+} \times J$ and compensators $\nu^{k}$ respectively.

\subsubsection{Random measures: tangency and decoupling}

It turns out that Cox processes play an important rôle in random measure theory and in particular if one changes a random measure by the corresponding Cox 
process then the strong $L^{p}$-norm does not change much. Recall that a stochastic integral with respect to a random measure is defined by (2.10).

Theorem 3.22. Let $X$ be a Banach space, $1 \leq p<\infty$. Then $X$ is UMD if and only if for any measurable space $(J, \mathcal{J})$ and any integer-valued random measure $\mu$ on $\mathbb{R}_{+} \times J$ with a compensator measure $\nu$ which is non-atomic in time one has that for any elementary predictable $F: \mathbb{R}_{+} \times \Omega \times J \rightarrow X$

$$
\mathbb{E} \sup _{t \geq 0}\left\|\int_{[0, t] \times J} F \mathrm{~d} \bar{\mu}\right\|^{p} \bar{\sim}_{p, X} \mathbb{E}\left\|\int_{\mathbb{R}_{+} \times J} F \mathrm{~d} \bar{\mu}_{\operatorname{Cox}}\right\|^{p},
$$

where $\mu_{\mathrm{Cox}}$ is the Cox process directed by $\nu, \bar{\mu}=\mu-\nu$, and $\bar{\mu}_{\mathrm{Cox}}=\mu_{\mathrm{Cox}}-\nu$.

For the proof we will need the following proposition. Recall that a Poisson measure $N$ is called nontrivial if its compensator in nonzero (equivalently, if it is nonzero itself).

Proposition 3.23. Let $X$ be a Banach space, $1 \leq p<\infty,(J, \mathcal{J})$ be a measurable space, $N$ be a nontrivial Poisson random measure on $\mathbb{R}_{+} \times J$ with a compensator $\nu=\lambda \otimes \kappa$ with $\lambda$ being Lebesgue on $\mathbb{R}_{+}$and $\kappa$ being a $\sigma$-finite measure on $(J, \mathcal{J}), \widetilde{N}:=N-\nu$ be the corresponding compensated Poisson measure. Then $X$ has the UMD property if and only if for any elementary predictable $F: \mathbb{R}_{+} \times \Omega \times J \rightarrow X$

$$
\mathbb{E} \sup _{t \geq 0}\left\|\int_{[0, t] \times J} F \mathrm{~d} \widetilde{N}\right\|^{p} \bar{\sim}_{p, X} \mathbb{E}\left\|\int_{\mathbb{R}_{+} \times J} F \mathrm{~d} \widetilde{N}_{\text {ind }}\right\|^{p}
$$

where $\widetilde{N}_{\text {ind }}$ is an independent copy of $\widetilde{N}$.

Proof. First notice that as $\nu=\lambda \otimes \kappa, \widetilde{N}$ is time-homogeneous, i.e. the distributions of $\widetilde{N}$ and shifted $\widetilde{N}(\cdot, \cdot+t, \cdot)$ are the same for any $t \geq 0$.

The "only if" part follows from the inequalities (1.2) for discrete tangent martingales, Remark 2.28, the definition of a stochastic integral with reapect to a random measure (2.10), and from the fact that by [47, Proposition 6.1.12]

$$
\begin{aligned}
\mathbb{E}\left\|\int_{\mathbb{R}_{+} \times J} F \mathrm{~d} \widetilde{N}_{\text {ind }}\right\|^{p}=\mathbb{E} \mathbb{E}_{N_{\text {ind }}}\left\|\int_{\mathbb{R}_{+} \times J} F \mathrm{~d} \widetilde{N}_{\text {ind }}\right\|^{p} \\
\bar{\sim}_{p} \mathbb{E} \mathbb{E}_{N_{\text {ind }}} \sup _{t \geq 0}\left\|\int_{[0, t] \times J} F \mathrm{~d} \widetilde{N}_{\text {ind }}\right\|^{p}=\mathbb{E} \sup _{t \geq 0}\left\|\int_{[0, t] \times J} F \mathrm{~d} \widetilde{N}_{\text {ind }}\right\|^{p}
\end{aligned}
$$

(here $\mathbb{E}_{N_{\text {ind }}}$ is defined by Example 2.5). Let us show the "if" part. Let (3.11) be satisfied for some $1 \leq p<\infty$ and for any elementary predictable $F$. Then

$$
\begin{aligned}
\mathbb{E} \sup _{t \geq 0}\left\|\int_{[0, t] \times J} F \mathrm{~d} \widetilde{N}\right\|^{p} & \bar{\sim}_{p, X} \mathbb{E}\left\|\int_{\mathbb{R}_{+} \times J} F \mathrm{~d} \widetilde{N}_{\text {ind }}\right\|^{p} \\
& \stackrel{(*)}{\sim}{ }_{p} \mathbb{E}\left\|\int_{\mathbb{R}_{+} \times J} F \mathrm{~d} \widetilde{N}_{\text {ind }}^{1}-\int_{\mathbb{R}_{+} \times J} F \mathrm{~d} \widetilde{N}_{\text {ind }}^{2}\right\|^{p},
\end{aligned}
$$


where $\widetilde{N}_{\text {ind }}^{1}$ and $\widetilde{N}_{\text {ind }}^{2}$ are independent copies of $\widetilde{N}$, and $(*)$ follows by a triangle inequality and the $L^{p}$-contractility of a conditional expectation [46, Corollary 2.6.30]. Therefore for any predictable process $a: \mathbb{R}_{+} \times \Omega \rightarrow\{-1,1\}$ independent of $\widetilde{N}_{\text {ind }}^{1}$ and $\widetilde{N}_{\text {ind }}^{2}$ one has that

$$
\begin{aligned}
\mathbb{E} \sup _{t \geq 0}\left\|\int_{[0, t] \times J} a F \mathrm{~d} \widetilde{N}\right\|^{p} & \bar{\sim}_{p, X} \mathbb{E}\left\|\int_{\mathbb{R}_{+} \times J} a F \mathrm{~d} \widetilde{N}_{\text {ind }}^{1}-\int_{\mathbb{R}_{+} \times J} a F \mathrm{~d} \widetilde{N}_{\text {ind }}^{2}\right\|^{p} \\
& \stackrel{(*)}{=} \mathbb{E}\left\|\int_{\mathbb{R}_{+} \times J} F \mathrm{~d} \widetilde{N}_{\text {ind }}^{1}-\int_{\mathbb{R}_{+} \times J} F \mathrm{~d} \widetilde{N}_{\text {ind }}^{2}\right\|^{p} \\
& \bar{\sim}_{p, X} \mathbb{E} \sup _{t \geq 0}\left\|\int_{[0, t] \times J} F \mathrm{~d} \widetilde{N}\right\|^{p}
\end{aligned}
$$

where $(*)$ follows from the fact that we are integrating both $a F$ and $F$ with respect to a symmetric independent noise, so $a$ does not play any role. Let us show that (3.12) implies the UMD property. Without loss of generality by assuming that $J:=A$ for some fixed $A \subset \mathcal{J}$ with $0<\kappa(A)<\infty$ and that $F$ has only steps of the form $\mathbf{1}_{A}$, we may assume that $J$ consists only of one point and thus $\widetilde{N}$ is a standard compensated Poisson process with the rate parameter $\kappa(A)$ (so, by a time-change argument the rate can be assumed 1 ), and thus (3.12) implies that for any elementary predictable $F: \mathbb{R}_{+} \times \Omega \rightarrow X$

$$
\mathbb{E} \sup _{t \geq 0}\left\|\int_{0}^{t} a F \mathrm{~d} \widetilde{N}\right\|^{p} \bar{\sim}_{p, X} \mathbb{E} \sup _{t \geq 0}\left\|\int_{0}^{t} F \mathrm{~d} \widetilde{N}\right\|^{p}
$$

First assume that $p>1$. Then due to Doob's maximal inequality (2.6), (3.13) is equivalent to

$$
\mathbb{E}\left\|\int_{0}^{\infty} a F \mathrm{~d} \widetilde{N}\right\|^{p} \bar{\sim}_{p, X} \mathbb{E}\left\|\int_{0}^{\infty} F \mathrm{~d} \widetilde{N}\right\|^{p} .
$$

Let $\left(r_{n}\right)_{n=1}^{N}$ be a sequence of independent Rademacher random variables (see Definition 2.1), $\phi_{1} \in X, \phi_{n}:\{-1,1\}^{n-1} \rightarrow X$ for $2 \leq n \leq N$. Let $\left(\varepsilon_{n}\right)_{n=1}^{N}$ be a $\{-1,1\}$-valued sequence. By the definition of the UMD property and by $[46$, Theorem 4.2.5] we only need to show that

$$
\mathbb{E}\left\|\sum_{n=1}^{N} \varepsilon_{n} r_{n} \phi_{n}\left(r_{1}, \ldots, r_{n-1}\right)\right\|^{p} \lesssim_{p, X} \mathbb{E}\left\|\sum_{n=1}^{N} r_{n} \phi_{n}\left(r_{1}, \ldots, r_{n-1}\right)\right\|^{p} .
$$

To this end we approximate in $L^{p}$-sense distributions of $\sum_{n=1}^{N} r_{n} \phi_{n}\left(r_{1}, \ldots, r_{n-1}\right)$ and $\sum_{n=1}^{N} \varepsilon_{n} r_{n} \phi_{n}\left(r_{1}, \ldots, r_{n-1}\right)$ by $\int_{0}^{\infty} F \mathrm{~d} \widetilde{N}$ and $\int_{0}^{\infty} a F \mathrm{~d} \widetilde{N}$ respectively by finding appropriate $F$ and $a$.

Fix $\varepsilon>0$. Let $A>0$ be such that for a stopping time

$$
\tau:=\inf \left\{t \geq 0:\left|\widetilde{N}_{t}\right| \geq A\right\}
$$

one has that

$$
\left\|\operatorname{sign} \widetilde{N}_{\tau}-\widetilde{N}_{\tau} / A\right\|_{L^{p}(\Omega)}<\varepsilon
$$


Such $A$ exists since $\left|\Delta \widetilde{N}_{\tau}\right| \leq 1$ and $\tau<\infty$ a.s. as $\tilde{N}$ is unbounded a.s., so a.s.

$$
\tilde{N}_{\tau} / A \in\left[-1-\frac{1}{A},-1\right] \cup\left[1,1+\frac{1}{A}\right],
$$

and since by [56, Theorem 25.14]

$$
\mathbb{E} \widetilde{N}_{\tau}=0
$$

Let $\tau_{0}=0, \tau_{1}=\tau$, and for any $2 \leq n \leq N$ define

$$
\tau_{n}:=\inf \left\{t \geq \tau_{n-1}:\left|\widetilde{N}_{t}-\widetilde{N}_{\tau_{n-1}}\right| \geq A\right\} .
$$

By strong Markov property of Lévy processes we have that $\left(\widetilde{N}_{\tau_{n}}-\widetilde{N}_{\tau_{n-1}}\right)_{n=1}^{N}$ are independent, and thus by (3.16) and (3.18) there exists a sequence of independent Rademacher random variables which we without loss of generality can denote by $\left(r_{n}\right)_{n=1}^{N}$ such that

$$
\left\|r_{n}-\left(\tilde{N}_{\tau_{n}}-\tilde{N}_{\tau_{n}-1}\right) / A\right\|_{L^{p}(\Omega)} \lesssim_{p} \varepsilon, \quad 1 \leq n \leq N .
$$

(One just needs to use the fact that by (3.16) and (3.18), $\left|\mathbb{E} \operatorname{sign}\left(N_{\tau_{n}}-N_{\tau_{n-1}}\right)\right| \lesssim_{p}$ $\varepsilon$, so by (3.17) one can approximate $\left(\widetilde{N}_{\tau_{n}}-\widetilde{N}_{\tau_{n}-1}\right) / A$ by a Rademacher.) Now let us define appropriate $F$ and $a$ in the following way:

$$
\begin{gathered}
F(t):= \begin{cases}\phi_{1} / A, & \text { if } 0 \leq t \leq \tau_{1}, \\
\phi_{n}\left(r_{1}, \ldots, r_{n-1}\right) / A, & \text { if } \tau_{n-1}<t \leq \tau_{n}, 2 \leq n \leq N, \\
0, & \text { if } t>\tau_{N},\end{cases} \\
a(t):= \begin{cases}\varepsilon_{n}, & \text { if } \tau_{n-1}<t \leq \tau_{n}, 1 \leq n \leq N, \\
1, & \text { if } t>\tau_{N} .\end{cases}
\end{gathered}
$$

Then one has that $F$ and $a$ are predictable by [52, Theorem I.2.2], and moreover

$$
\begin{aligned}
\| \int_{0}^{\infty} F \mathrm{~d} \widetilde{N} & -\sum_{n=1}^{N} r_{n} \phi_{n}\left(r_{1}, \ldots, r_{n-1}\right) \|_{L^{p}(\Omega ; X)} \\
& =\left\|\sum_{n=1}^{N}\left(r_{n}-\left(\widetilde{N}_{\tau_{n}}-\widetilde{N}_{\tau_{n}-1}\right) / A\right) \phi_{n}\left(r_{1}, \ldots, r_{n-1}\right)\right\|_{L^{p}(\Omega ; X)} \\
& \leq \sum_{n=1}^{N}\left\|\left(r_{n}-\left(\widetilde{N}_{\tau_{n}}-\widetilde{N}_{\tau_{n}-1}\right) / A\right) \phi_{n}\left(r_{1}, \ldots, r_{n-1}\right)\right\|_{L^{p}(\Omega ; X)} \\
& \leq L \sum_{n=1}^{N}\left\|r_{n}-\left(\widetilde{N}_{\tau_{n}}-\tilde{N}_{\tau_{n}-1}\right) / A\right\|_{L^{p}(\Omega ; X)} \stackrel{(*)}{\lesssim_{p}} N L \varepsilon,
\end{aligned}
$$

where $L>0$ is such that $\left\|\phi_{n}\right\|_{\infty}<L$ for any $1 \leq n \leq N$, and (*) follows from (3.19). For the same reason we have that

$$
\left\|\int_{0}^{\infty} a F \mathrm{~d} \tilde{N}-\sum_{n=1}^{N} \varepsilon_{n} r_{n} \phi_{n}\left(r_{1}, \ldots, r_{n-1}\right)\right\|_{L^{p}(\Omega ; X)} \lesssim_{p} N L \varepsilon .
$$

By letting $\varepsilon \rightarrow 0$ and by (3.14) we obtain (3.15). 
Now let $p=1$. Then we need to use good $-\lambda$ inequalities in order to show that (3.13) holds for any $p>1$ (see Section 5). Let $M=\int_{\left[0,{ }^{\prime}\right] \times J} F \mathrm{~d} \widetilde{N}$ and $L=\int_{[0, \cdot] \times J} a F \mathrm{~d} \tilde{N}$ for some elementary predictable $F: \mathbb{R}_{+} \times \Omega \rightarrow \mathbb{R}$ and $a: \mathbb{R}_{+} \times \Omega \rightarrow\{0,1\}$. Let us fix $\beta>1, \delta>0$, and $\lambda>0$, and let us define stopping times

$$
\begin{aligned}
& \sigma:=\inf \left\{t \geq 0:\left\|L_{t}\right\|>\lambda\right\} \\
& \tau:=\inf \left\{t \geq 0:\left\|M_{t}\right\|>\delta \lambda\right\} \\
& \rho:=\inf \left\{t \geq 0:\left\|F_{t}\right\|>\delta \lambda\right\}
\end{aligned}
$$

Define

$$
\begin{aligned}
\widehat{M}_{t} & :=\int_{[0, t] \times J} F \mathbf{1}_{(\tau \wedge \sigma \wedge \rho, \tau \wedge \rho]} \mathrm{d} \tilde{N}, \quad t \geq 0, \\
\widehat{L}_{t} & :=\int_{[0, t] \times X} a F \mathbf{1}_{(\tau \wedge \sigma \wedge \rho, \tau \wedge \rho]} \mathrm{d} \tilde{N}, \quad t \geq 0 .
\end{aligned}
$$

Note that $\widehat{M}$ coincides with $M-M^{\tau \wedge \sigma \wedge \rho}$ on $[0, \tau \wedge \rho]$, so by the definition of $\tau$ and $\rho$ we have that $\widehat{M} \leq 2 \delta \lambda$ (note that $F$ is elementary predictable, so $\rho$ is predictable, and so $\Delta M_{\rho}=\Delta L_{\rho}=0$ as $M$ and $L$ are quasi-left continuous), and thus by (3.13) for $p=1$ we have that

$$
\mathbb{E} \sup _{t \geq 0}\left\|\widehat{L}_{t}\right\| \lesssim X \mathbb{E} \sup _{t \geq 0}\left\|\widehat{M}_{t}\right\| \leq 2^{p} \delta^{p} \lambda^{p}
$$

Therefore, as $\left\|\Delta M_{t}\right\| \leq\left\|F_{t}\right\|$ a.s. for any $t \geq 0$,

$$
\begin{aligned}
\mathbb{P}\left(L^{*}>\beta \lambda, \Delta M^{*} \vee M^{*} \leq \delta \lambda\right) & \leq \mathbb{P}\left(L^{*}>\beta \lambda, \tau=\rho=\infty\right) \stackrel{(*)}{\leq} \mathbb{P}\left(\widehat{L}^{*}>(\beta-\delta-1) \lambda\right) \\
& \leq \frac{1}{(\beta-\delta-1) \lambda} \mathbb{E} \widehat{L}^{*} \stackrel{(* *)}{\lesssim} x \frac{1}{(\beta-\delta-1) \lambda} \mathbb{E} \widehat{M}^{*}
\end{aligned}
$$

where $(*)$ follows from the fact that if $\tau=\rho=\infty$, then $\widehat{L}$ coincides with $L-L^{\tau \wedge \sigma \wedge \rho}$ on $\mathbb{R}_{+}$, and the fact that $\mathbb{P}(\sigma=\rho)=0$ as $\rho$ is predictable and $\sigma$ is totally inaccessible (see [56, Chapter 25$]$ ), while $(* *)$ holds by $(3.20)$. On the other hand as $\widehat{M} \leq 2 \delta \lambda$ a.s.

$$
\mathbb{E} \widehat{M}^{*}=\mathbb{E} \widehat{M}^{*} \mathbf{1}_{\tau \wedge \sigma \wedge \rho<\infty} \leq 2 \delta \lambda \mathbb{P}(\sigma<\infty)=2 \delta \lambda \mathbb{P}\left(L^{*}>\lambda\right) .
$$

Consequently,

$$
\mathbb{P}\left(L^{*}>\beta \lambda, \Delta M^{*} \vee M^{*} \leq \delta \lambda\right) \lesssim X \frac{2 \delta \lambda}{(\beta-\delta-1) \lambda} \mathbb{P}\left(L^{*}>\lambda\right),
$$

and one has that (3.13) holds for any $p>1$ by Lemma 5.2 and by the fact that $\Delta M^{*} \leq 2 M^{*}$ a.s., so the UMD property follows from the case $p>1$ considered above. 
For the proof of Theorem 3.22 we will also need the following technical lemma on approximation of continuous increasing predictable functions which simpler form was proven in [32, Subsection 5.5].

Lemma 3.24. Let $F: \mathbb{R}_{+} \times \Omega \rightarrow \mathbb{R}_{+}$be a nondecreasing continuous predictable process such that $F(t)-F(s) \leq C(t-s)$ a.s. for any $0 \leq s \leq t$ and any $C>0$, and such that $F(0)=0$ a.s. Let $0<p<\infty$. Then for any $p \in(0,1]$, for any $T \geq 0$, and for any $\delta>0$ these exists natural $K_{0}>0$ such that for any $K>K_{0}$ and for $\left(t_{k}\right)_{k=0}^{K}=(T k / K)_{k=0}^{K}$ we have that

$$
\mathbb{E}\left(\sum_{k=1}^{K}\left|F\left(t_{k}\right)-\mathbb{E}\left(F\left(t_{k}\right) \mid \mathcal{F}_{t_{k-1}}\right)\right|\right)^{p}<\delta .
$$

Proof. Let us first show the lemma for $p=1$. As it was shown in [32, Subsection $5.5]$, there exists a predictable process $f: \mathbb{R}_{+} \times \Omega \rightarrow[0, C]$ such that a.s.

$$
F_{t}=\int_{0}^{t} f(s) \mathrm{d} s, \quad t \geq 0 .
$$

For each $K>0$ define

$$
T_{K} f(t):=\mathbb{E}\left(f(t) \mid \mathcal{F}_{t_{k}}\right), \quad t_{k-1}<t \leq t_{k}, \quad k=1, \ldots, K .
$$

Then it is sufficient to show that $T_{K} f$ converges to $f$ in $L^{1}\left([0, T] \times \Omega,\left.\lambda\right|_{[0, T]} \otimes \mathbb{P}\right)$ (where $\lambda$ is the Lebesgue measure on $\mathbb{R}_{+}$) as

$$
\begin{aligned}
\mathbb{E} \sum_{k=1}^{K}\left|F\left(t_{k}\right)-\mathbb{E}\left(F\left(t_{k}\right) \mid \mathcal{F}_{t_{k-1}}\right)\right| & =\mathbb{E} \sum_{k=1}^{K}\left|\int_{t_{k-1}}^{t_{k}} f(t)-\mathbb{E}\left(f(t) \mid \mathcal{F}_{t_{k}}\right) \mathrm{d} t\right| \\
& \leq \mathbb{E} \sum_{k=1}^{K} \int_{t_{k-1}}^{t_{k}}\left|f(t)-\mathbb{E}\left(f(t) \mid \mathcal{F}_{t_{k}}\right)\right| \mathrm{d} t \\
& =\mathbb{E} \int_{0}^{T}\left|f(t)-\mathbb{E}\left(f(t) \mid \mathcal{F}_{t_{k}}\right)\right| \mathrm{d} t \\
& \left.=\left\|f-T_{K} f\right\|_{L^{1}([0, T] \times \Omega, \lambda \mid[0, T]} \otimes \mathbb{P}\right) .
\end{aligned}
$$

Note that $T_{K}$ is a bounded linear operator on $L^{1}\left([0, T] \times \Omega,\left.\lambda\right|_{[0, T]} \otimes \mathbb{P}\right)$ of norm 1 as for any $g \in L^{1}\left([0, T] \times \Omega,\left.\lambda\right|_{[0, T]} \otimes \mathbb{P}\right)$ by the Fubini theorem and by the fact that a conditional expectation is a contraction on $L^{1}(\Omega)$

$$
\begin{aligned}
\left.\left\|T_{K} g\right\|_{L^{1}([0, T] \times \Omega, \lambda \mid[0, T]} \otimes \mathbb{P}\right) & =\mathbb{E} \int_{0}^{T}\left|\mathbb{E}\left(g(t) \mid \mathcal{F}_{t_{k}}\right)\right| \mathrm{d} t=\int_{0}^{T} \mathbb{E}\left|\mathbb{E}\left(g(t) \mid \mathcal{F}_{t_{k}}\right)\right| \mathrm{d} t \\
& \leq \int_{0}^{T} \mathbb{E}|g(t)| \mathrm{d} t=\mathbb{E} \int_{0}^{T}|g(t)| \mathrm{d} t=\|g\|_{L^{1}\left([0, T] \times \Omega,\left.\lambda\right|_{[0, T]}\right.} .
\end{aligned}
$$

Therefore by [34, Lemma 9.4.7] it is sufficient to show that $T_{k} f_{n} \rightarrow f_{n}$ for a converging to $f$ sequence $\left(f_{n}\right)_{n \geq 1}$. To this end we need to set $f_{n}(\cdot):=f(\cdot-1 / n)$ 
on $[1 / n, \infty]$ and $f(\cdot)=0$ on $[0,1 / n]$. Then $f_{n}$ converges to $f$ in $L^{1}([0, T] \times$ $\left.\Omega,\left.\lambda\right|_{[0, T]} \otimes \mathbb{P}\right)$ as $n \rightarrow \infty$ by [34, Lemma 9.4.7] (translation operators have a strong limit, namely the identity operator, see e.g. [4, Theorem 1]) and by the dominated convergence theorem, while $T_{K} f_{n}=f_{n}$ for $K \geq n$ as in this case $f_{n}(t)$ is $\mathcal{F}_{t-1 / K}$-measurable. Therefore the desired follows.

Let us show the case $p \neq 1$. In this case it is sufficient to notice that for any $K \geq 1$ a.s.

$$
\begin{aligned}
\sum_{k=1}^{K} & \left|F\left(t_{k}\right)-\mathbb{E}\left(F\left(t_{k}\right) \mid \mathcal{F}_{t_{k-1}}\right)\right| \\
& =\sum_{k=1}^{K}\left|F\left(t_{k}\right)-F\left(t_{k-1}\right)-\mathbb{E}\left(F\left(t_{k}\right)-F\left(t_{k-1}\right) \mid \mathcal{F}_{t_{k-1}}\right)\right| \\
& \stackrel{(i)}{\leq} \sum_{k=1}^{K}\left|F\left(t_{k}\right)-F\left(t_{k-1}\right)\right|+\left|\mathbb{E}\left(F\left(t_{k}\right)-F\left(t_{k-1}\right) \mid \mathcal{F}_{t_{k-1}}\right)\right| \\
& \stackrel{(i i)}{=} \sum_{k=1}^{K} F\left(t_{k}\right)-F\left(t_{k-1}\right)+\mathbb{E}\left(F\left(t_{k}\right)-F\left(t_{k-1}\right) \mid \mathcal{F}_{t_{k-1}}\right) \\
& \stackrel{(i i i)}{=} \int_{0}^{T} f(t) \mathrm{d} t+\int_{0}^{T} T_{K} f(t) \mathrm{d} t \stackrel{(i v)}{\leq} 2 C T,
\end{aligned}
$$

where $f$ is defined by $(3.22), T_{K}$ is defined by $(3.23),(i)$ follows from a triangle inequality, (ii) follows from the fact that $F$ is nondecreasing (and the same holds for the conditional expectations), (iii) follows from the definition of $f$ and $T_{k} f$, and $(i v)$ holds by the fact that $f \in[0, C]$ a.e. on $\mathbb{R}_{+} \times \Omega$, by the definition (3.22) of $T_{K}$, and the fact that a conditional expectation is a contraction on $L^{\infty}$ (so $T_{K} f \in[0, C]$ a.e. on $\left.\mathbb{R}_{+} \times \Omega\right)$. Therefore (3.21) for $p \neq 1$ follows by the dominated convergence theorem and the case $p=1$.

Proof of Theorem 3.22. The "if" part follows from Proposition 3.23. Let us show the "only if" part. As $F$ is elementary predictable we may assume that $J$ is finite, $J=\{1, \ldots, n\}, \mathcal{J}$ is generated by all atoms, $X$ is finite dimensional, and $F$ has the following form

$$
F(t, \cdot, j)=\sum_{k=1}^{K} \mathbf{1}_{\left[t_{k-1}, t_{k}\right)}(t) \xi_{k, j}, \quad t \geq 0,1 \leq j \leq n,
$$

where $0 \leq t_{0} \leq \ldots \leq t_{K}$, and $\xi_{k, j}$ is elementary $X$-valued $\mathcal{F}_{t_{k-1}}$-measurable for any $k=1, \ldots, K$ and $1 \leq j \leq n$.

Let $\mu_{\text {Cox }}$ be as constructed in Example 3.21. Then we need to show that

$$
\begin{aligned}
\mathbb{E} \sup _{t \geq 0}\left\|\sum_{k=1}^{K} \sum_{j=1}^{n} \bar{\mu}\left(\left[t_{k-1} \wedge t, t_{k} \wedge t\right) \times\{j\}\right) \xi_{k, j}\right\|^{p} \\
\quad \bar{\sim}_{p, X} \mathbb{E} \mathbb{E}_{N} \sup _{t \geq 0}\left\|\sum_{k=1}^{K} \sum_{j=1}^{n} \widetilde{N}\left(\left[\nu^{j}\left(\left[0, t_{k-1} \wedge t\right)\right), \nu^{j}\left(\left[0, t_{k} \wedge t\right)\right)\right) \times\{j\}\right) \xi_{k, j}\right\|^{p},
\end{aligned}
$$


where $\nu_{N}$ is a compensator of $N, \widetilde{N}:=N-\nu_{N}, \mathbb{E}_{N}$ denotes expectation in $\Omega_{N}$ (i.e. the expectation taken for a fixed $\omega \in \Omega$, see Example 2.5), and $\nu^{j}$ is a random measure on $\mathbb{R}_{+}$of the form

$$
\nu^{j}(A):=\nu(A \times\{j\}), \quad A \in \mathcal{B}\left(\mathbb{R}_{+}\right), \quad j=1, \ldots, n .
$$

In order to derive (3.25) we will use the fact that any random measure is Poisson after a certain time-change (see [56, Corollary 25.26]) and the decoupling inequality (3.11). The proof will be done in four steps.

Step 1: $\nu([s, t) \times\{j\}) \leq t-s, \nu\left(\mathbb{R}_{+} \times\{j\}\right)=\infty, 1 \leq p \leq 2$. First assume that a.s.

$$
\nu([s, t] \times J) \leq t-s, \quad 0 \leq s \leq t,
$$

that $\nu\left(\mathbb{R}_{+} \times\{j\}\right)=\infty$ a.s. for any $j=1, \ldots, n$, and that $1 \leq p \leq 2$. By the fact that any martingale has a càdlàg version (see Subsection 2.5) and by adding knots to the mesh we may assume that $K$ is so big that (or the mesh is so small that)

$$
\mathbb{E} \max _{k=1}^{K}\left\|\int_{\left[0, t_{k}\right] \times J} F \mathrm{~d} \bar{\mu}\right\|^{p} \leq \mathbb{E} \sup _{t \geq 0}\left\|\int_{[0, t] \times J} F \mathrm{~d} \bar{\mu}\right\|^{p} \leq 2 \mathbb{E} \max _{k=1}^{K}\left\|\int_{\left[0, t_{k}\right] \times J} F \mathrm{~d} \bar{\mu}\right\|^{p},
$$

so instead of (3.10) it is sufficient to show that for $K$ big enough

$$
\mathbb{E} \max _{k=1}^{K}\left\|\int_{\left[0, t_{k}\right] \times J} F \mathrm{~d} \bar{\mu}\right\|^{p} \bar{\sim}_{p, X} \mathbb{E}\left\|\int_{\mathbb{R}_{+} \times J} F \mathrm{~d} \bar{\mu}_{\operatorname{Cox}}\right\|^{p} .
$$

By [56, Corollary 25.26] the random measure $\chi$ defined on $\mathbb{R}_{+} \times \Omega$ by

$$
\chi([0, s) \times\{j\}):=\mu\left(\left[0, \tau_{s}^{j}\right) \times\{j\}\right), \quad s \geq 0, \quad 1 \leq j \leq n,
$$

with

$$
\tau_{s}^{j}:=\inf \{s \geq 0: \nu([0, s) \times\{j\}) \geq t\}, \quad s \geq 0,
$$

is a standard Poisson random measure with a compensator

$$
\nu_{\chi}([0, s) \times\{j\})=s, \quad s \geq 0, \quad 1 \leq j \leq n .
$$

Without loss of generality by an approximation argument we may assume that $K$ in (3.24) is so large so that there exists $T>0$ such that $t_{0}, \ldots, t_{K}$ in (3.24) are $0, \frac{T}{K}, \ldots, \frac{T(k-1)}{K}, T$. Moreover, by considering a smaller mash for any $\delta>0$ we can assume that $K$ is so large that by Lemma 3.24, by predictability and continuity of the process $t \mapsto \nu([0, t))$, and by $(3.27)$

$$
\mathbb{E} \max _{k=1}^{K} \sum_{j=1}^{n}\left|\nu\left(\left[0, t_{k}\right) \times\{j\}\right)-\mathbb{E}\left(\nu\left(\left[0, t_{k}\right) \times\{j\}\right) \mid \mathcal{F}_{t_{k-1}}\right)\right|<\delta .
$$

Therefore the integral on the left-hand side of (3.25) becomes

$$
t \mapsto \sum_{k=1}^{K} \sum_{j=1}^{n} \bar{\chi}\left(\left[\nu^{j}\left(\left[0, t_{k-1} \wedge t\right)\right), \nu^{j}\left(\left[0, t_{k} \wedge t\right)\right)\right) \times\{j\}\right) \xi_{k, j}
$$


where $\bar{\chi}=\chi-\nu_{\chi}$. As $\chi$ is a standard Poisson random measure, by (3.27), by adding some pieces of standard Poisson random measure within stopping times, and by using the fact that Poisson process is strong Markov and stationary, without loss of generality we may assume that there exists a standard Poisson random measure $\eta$ on $\mathbb{R}_{+} \times J$ with a compensator measure $\nu_{\eta}=\nu_{\chi}$ such that

$$
\left.\eta\right|_{\left.t_{k-1}, t_{k-1}+\nu^{j}\left[t_{k-1}, t_{k}\right)\right)}=\left.\chi\right|_{\left[\nu^{j}\left(\left[0, t_{k-1}\right)\right), \nu^{j}\left(\left[0, t_{k}\right)\right)\right)}, \quad k=1, \ldots, K,
$$

and $\left.\eta\right|_{\left[t_{k-1}+\nu^{j}\left[t_{k-1}, t_{k}\right), t_{k}\right)}$ is copied from an independent from $\chi$ standard Poisson random measure. Then the integral above becomes as follows

$$
M_{t}=\sum_{k=1}^{K} \sum_{j=1}^{n} \bar{\eta}\left(\left[t_{k-1}, t_{k-1}+\nu^{j}\left[t_{k-1} \wedge t, t_{k} \wedge t\right)\right) \times\{j\}\right) \xi_{k, j}, \quad t \geq 0,
$$

where $\xi_{k, j}$ is $\mathcal{F}_{t_{k-1}} \otimes \sigma\left(\left.\eta\right|_{\left[0, t_{k-1}\right]}\right)$-measurable and $\bar{\eta}:=\eta-\nu_{\eta}$. Let $L: \mathbb{R}_{+} \times \Omega \rightarrow X$ be a process defined for every $t \geq 0$ by

$$
L_{t}=\sum_{k=1}^{K} \sum_{j=1}^{n} \bar{\eta}\left(\left[t_{k-1} \wedge t,\left(t_{k-1}+\mathbb{E}\left(\nu^{j}\left[t_{k-1}, t_{k}\right) \mid \mathcal{F}_{t_{k-1}}\right)\right) \wedge t\right) \times\{j\}\right) \xi_{k, j}
$$

Note that $L$ is a martingale with respect to an enlarged filtration $\mathbb{F}^{\prime}=\left(\mathcal{F}_{t}^{\prime}\right)_{t \geq 0}$ of the following form

$$
\mathcal{F}_{t}^{\prime}= \begin{cases}\sigma\left(\left.\eta\right|_{[0, t]}, \mathcal{F}_{0}\right), & 0 \leq t<t_{1}, \\ \sigma\left(\left.\eta\right|_{[0, t]}, \mathcal{F}_{t_{k}}^{\prime}\right), & 1 \leq k<K, \quad t_{k}<t<t_{k+1}, \\ \sigma\left(\left.\eta\right|_{\left[0, t_{k}\right]}, \mathcal{F}_{t_{k}}\right), & 1 \leq k \leq K, \quad t=t_{k}, \\ \mathcal{F}_{t_{K}}^{\prime}, & t>t_{K} .\end{cases}
$$

$L$ is a martingale with respect to $\mathbb{F}^{\prime}$, but it can be decomposed into two parts $L^{1}$ and $L^{2}$ which are martingales in different filtrations, in the following way. First we introduce a stopping time

$$
\sigma_{k}^{j}:=\tau_{\nu^{j}\left[0, t_{k-1}\right)+\mathbb{E}\left(\nu^{j}\left[t_{k-1}, t_{k}\right) \mid \mathcal{F}_{t_{k-1}}\right)},
$$

where $\tau_{s}^{j}$ is as defined by (3.29). Then let us define for any $t \geq 0$

$$
\begin{aligned}
L_{t}^{1} & :=\sum_{k=1}^{K} \sum_{j=1}^{n} \bar{\eta}\left(\left[t_{k-1}, t_{k-1}+\mathbb{E}\left(\nu^{j}\left[t_{k-1}, t_{k}\right) \mid \mathcal{F}_{t_{k-1}}\right) \wedge \nu^{j}\left[t_{k-1} \wedge t, t_{k} \wedge t\right)\right) \times\{j\}\right) \xi_{k, j} \\
& =\sum_{k=1}^{K} \sum_{j=1}^{n} \bar{\mu}\left(\left[t_{k-1} \wedge t, \sigma_{k}^{j} \wedge t\right) \times\{j\}\right) \xi_{k, j},
\end{aligned}
$$

which is a martingale with respect to the original filtration, and 


$$
\begin{aligned}
L_{t}^{2}:=\sum_{k=1}^{K} \mathbf{1}_{t \geq t_{k}} \sum_{j=1}^{n} \bar{\eta}( & \left(\left[t_{k-1}+\nu^{j}\left[t_{k-1}, t_{k}\right)\right) \wedge t\right. \\
& \left.\left.\left(t_{k-1}+\mathbb{E}\left(\nu^{j}\left[t_{k-1}, t_{k}\right) \mid \mathcal{F}_{t_{k-1}}\right)\right) \wedge t\right) \times\{j\}\right) \mathbf{1}_{\sigma_{k}^{j} \geq t_{k}} \xi_{k, j},
\end{aligned}
$$

which is a martingale with respect to the enlarged filtration

$$
\mathbb{F}^{\prime \prime}=\left(\mathcal{F}_{t}^{\prime \prime}\right)_{t \geq 0}:=\left(\mathcal{F}_{\infty} \otimes \sigma\left(\left.\eta\right|_{A_{\eta} \cap[0, t] \times J}\right)\right)_{t \geq 0},
$$

where $A_{\eta}:=\cup_{k=1}^{K} \cup_{j \in J}\left[t_{k-1}, t_{k-1}+\nu^{j}\left[t_{k-1} \wedge t, t_{k} \wedge t\right)\right) \times\{j\}$ is a $\sigma$-field depending on $\Omega$ and $\otimes$ does not mean a direct product, see Subsection 2.2. Note that $L^{1}$ and $L^{2}$ are martingales in different scales, so $L=L^{1}+L^{2}$ not necessarily in general (unless $\nu((s, t] \times\{j\})=t-s$ ), but $L_{\infty}=L_{\infty}^{1}+L_{\infty}^{2}$ and $\sup _{t \geq 0}\left\|L_{t}\right\| \leq \sup _{t \geq 0}\left\|L_{t}^{1}\right\|+\sup _{t \geq 0}\left\|L_{t}^{2}\right\|$ a.s.

Next, by Novikov's inequalities (2.13), the fact that $X$ can be assumed finite dimensional, the fact that $F$ is uniformly bounded, the definition (3.33) of $\sigma_{k}^{j}$, and $(3.30)$

$$
\begin{aligned}
\underset{\mathbb{E} \max _{k=1}^{K}}{ }\left\|\int_{\left[0, t_{k}\right] \times J} F \mathrm{~d} \bar{\mu}-L_{t_{k}}^{1}\right\|^{p} \\
\quad=\mathbb{E} \max _{k=1}^{K}\left\|\sum_{\ell=1}^{k} \sum_{j=1}^{n} \bar{\mu}\left(\left[\sigma_{\ell}^{j}, t_{\ell}\right) \times\{j\}\right) \mathbf{1}_{\sigma_{\ell}^{j} \leq t_{\ell}} \xi_{\ell, j}\right\|^{p} \\
\quad \lesssim_{p, F} \mathbb{E} \sum_{k=1}^{K} \sum_{j=1}^{n} \nu\left(\left[\sigma_{k}^{j}, t_{k}\right) \times\{j\}\right) \mathbf{1}_{\sigma_{k}^{j} \leq t_{k}} \\
\leq \mathbb{E} \sum_{k=1}^{K} \sum_{j=1}^{n}\left|\nu^{j}\left(\left[0, t_{k}\right)\right)-\mathbb{E}\left(\nu^{j}\left(\left[0, t_{k}\right)\right) \mid \mathcal{F}_{t_{k-1}}\right)\right|<\delta .
\end{aligned}
$$

On the other hand for a similar reason and the fact that $\nu_{\eta}(\cdot \times\{j\})$ is a standard Lebesgue measure on $\mathbb{R}_{+}$for any $j=1, \ldots, n$

$$
\begin{aligned}
& \mathbb{E} \sup _{t \geq 0}\left\|L_{t}^{2}\right\|^{p}=\mathbb{E} \sup _{t \geq 0} \mathbb{E}_{\eta} \| \sum_{k=1}^{K} \mathbf{1}_{t \geq t_{k}} \sum_{j=1}^{n} \bar{\eta}\left(\left[t_{k-1}+\nu^{j}\left[t_{k-1}, t_{k}\right), t_{k-1}\right.\right. \\
& \left.\left.+\mathbb{E}\left(\nu^{j}\left[t_{k-1}, t_{k}\right) \mid \mathcal{F}_{t_{k-1}}\right)\right) \times\{j\}\right) \mathbf{1}_{\sigma_{k}^{j} \geq t_{k}} \xi_{k, j} \|^{p} \\
& \stackrel{(*)}{\lesssim}_{p, F} \mathbb{E} \sum_{k=1}^{K} \sum_{j=1}^{n} \nu_{\eta}\left(\left[t_{k-1}+\nu^{j}\left[t_{k-1}, t_{k}\right), t_{k-1}\right.\right. \\
& \left.\left.\left.+\mathbb{E}\left(\nu^{j}\left[t_{k-1}, t_{k}\right) \mid \mathcal{F}_{t_{k-1}}\right)\right)\right) \times\{j\}\right) \mathbf{1}_{\sigma_{k}^{j} \geq t_{k}} \\
& \left.\left.\leq \mathbb{E} \sum_{k=1}^{K} \sum_{j=1}^{n} \mid \mathbb{E}\left(\nu^{j}\left[t_{k-1}, t_{k}\right) \mid \mathcal{F}_{t_{k-1}}\right)\right)\right)-\nu^{j}\left[t_{k-1}, t_{k}\right) \mid \mathbf{1}_{\sigma_{k}^{j} \geq t_{k}} \\
& \leq \mathbb{E} \sum_{k=1}^{K} \sum_{j=1}^{n}\left|\nu^{j}\left(\left[0, t_{k}\right)\right)-\mathbb{E}\left(\nu^{j}\left(\left[0, t_{k}\right)\right) \mid \mathcal{F}_{t_{k-1}}\right)\right|<\delta,
\end{aligned}
$$


where $\mathbb{E}_{\eta}$ is defined by Example 2.5, (*) follows from the fact that $F$ is uniformly bounded, (2.13), and the fact that the random measure constructed from

$$
\left(\left.\eta\right|_{\cup_{j \in J}\left[t_{k-1}+\nu^{j}\left[t_{k-1}, t_{k}\right), t_{k}\right) \times\{j\}}\right)_{k=1}^{K}
$$

is standard Poisson with the compensator measure

$\left(\left.\nu_{\eta}\right|_{\cup_{j \in J}\left[t_{k-1}+\nu^{j}\left[t_{k-1}, t_{k}\right), t_{k}\right) \times\{j\}}\right)_{k=1}^{K}$. As we can choose $K$ big enough (and $\delta$ small enough), it is sufficient to show that

$$
\mathbb{E} \max _{k=1}^{K}\left\|L_{t_{k}}^{1}\right\|^{p} \bar{\sim}_{p, X} \mathbb{E}\left\|\int_{\mathbb{R}_{+} \times J} F \mathrm{~d} \bar{\mu}_{\operatorname{Cox}}\right\|^{p}
$$

To this end first notice that analogously to (3.35)

$$
\begin{aligned}
\mathbb{E E}_{N} \sup _{t \geq 0} \| & \sum_{k=1}^{K} \mathbf{1}_{t \geq t_{k}} \sum_{j=1}^{n} \tilde{N}\left(\left[t_{k-1}+\nu^{j}\left[t_{k-1} \wedge t, t_{k} \wedge t\right), t_{k-1}\right.\right. \\
& \left.\left.+\mathbb{E}\left(\nu^{j}\left[t_{k-1} \wedge t, t_{k} \wedge t\right) \mid \mathcal{F}_{t_{k-1}}\right)\right) \times\{j\}\right) \mathbf{1}_{\sigma_{k}^{j} \geq t_{k}} \xi_{k, j} \|^{p} \lesssim_{{ }_{p}, F} \delta,
\end{aligned}
$$

where $N$ is defined as in (3.25). Next note that by Theorem 1.1 (see also the proof of Proposition 3.23), (3.36), and the fact that $\mathbb{E}\left(\nu^{j}\left[t_{k-1} \wedge t, t_{k} \wedge t\right) \mid \mathcal{F}_{t_{k-1}}\right)$ is $\mathcal{F}_{t_{k-1}}^{\prime}$-measurable for any $t \geq 0$

$$
\begin{aligned}
& \mathbb{E} \max _{k=1}^{K}\left\|L_{t_{k}}^{1}\right\|^{p} \\
& =\mathbb{E} \max _{k=1}^{K} \mathbb{E}_{\eta} \| \sum_{\ell=1}^{k} \sum_{j=1}^{n} \bar{\eta}\left(\left[t_{\ell-1}, t_{\ell-1}+\mathbb{E}\left(\nu^{j}\left[t_{\ell-1}, t_{k}\right) \mid \mathcal{F}_{t_{\ell-1}}\right)\right) \times\{j\}\right) \mathbf{1}_{A_{\ell, j}} \xi_{\ell, j} \\
& +\sum_{\ell=1}^{k} \sum_{j=1}^{n} \bar{\eta}\left(\left[t_{\ell-1}, t_{\ell}\right) \times\{j\}\right) \mathbf{1}_{\bar{A}_{\ell, j}} \xi_{\ell, j} \|^{p} \\
& \bar{\sim}_{p, X} \mathbb{E} \mathbb{E}_{N} \max _{k=1}^{K} \| \sum_{\ell=1}^{k} \sum_{j=1}^{n} \widetilde{N}\left(\left[t_{\ell-1}, t_{\ell-1}+\mathbb{E}\left(\nu^{j}\left[t_{\ell-1}, t_{k}\right) \mid \mathcal{F}_{t_{\ell-1}}\right)\right) \times\{j\}\right) \mathbf{1}_{A_{\ell, j}} \xi_{\ell, j} \\
& +\sum_{\ell=1}^{k} \sum_{j=1}^{n} \tilde{N}\left(\left[t_{\ell-1}, t_{\ell}\right) \times\{j\}\right) \mathbf{1}_{A_{\ell, j}} \xi_{\ell, j} \|^{p} \\
& \stackrel{(*)}{\sim} \mathbb{E} \mathbb{E}_{N} \| \sum_{k=1}^{K} \sum_{j=1}^{n} \tilde{N}\left(\left[t_{k-1}, t_{k-1}+\mathbb{E}\left(\nu^{j}\left[t_{k-1}, t_{k}\right) \mid \mathcal{F}_{t_{k-1}}\right)\right) \times\{j\}\right) \mathbf{1}_{A_{k, j}} \xi_{k, j} \\
& +\sum_{k=1}^{K} \sum_{j=1}^{n} \tilde{N}\left(\left[t_{k-1}, t_{k}\right) \times\{j\}\right) \mathbf{1}_{\overline{A_{k, j}}} \xi_{k, j} \|^{p} \\
& \stackrel{(* *)}{\sim} \mathbb{E}_{\delta} \mathbb{E}_{N}\left\|\sum_{k=1}^{K} \sum_{j=1}^{n} \widetilde{N}\left(\left[t_{k-1}, t_{k-1}+\nu^{j}\left[t_{k-1}, t_{k}\right)\right) \times\{j\}\right) \xi_{k, j}\right\|^{p}
\end{aligned}
$$




$$
=\mathbb{E}\left\|\int_{\mathbb{R}_{+} \times J} F \mathrm{~d} \bar{\mu}_{\operatorname{Cox}}\right\|^{p}
$$

where $A_{k, j}:=\left\{\mathbb{E}\left(\nu^{j}\left[t_{k-1}, t_{k}\right) \mid \mathcal{F}_{t_{k-1}}\right) \leq \nu^{j}\left[t_{k-1}, t_{k}\right)\right\} \subset \Omega,(*)$ holds by Lemma 9.3 , and $(* *)$ holds for $\delta$ small enough by (3.30) e.g. analogously (3.34). Therefore (3.28), and hence (3.10), follows. This terminates the proof.

Step 2: $\nu([s, t) \times\{j\}) \leq t-s, \nu\left(\mathbb{R}_{+} \times\{j\}\right)=\infty$, general $1 \leq p<\infty$. In the case of a general $1 \leq p<\infty$ we will have exactly the same proof as in Step 1 , but with applying more complicated Novikov inequalities (2.13) for the case $p>2$.

Step 3: $\nu([s, t) \times\{j\})<\infty, \nu\left(\mathbb{R}_{+} \times\{j\}\right)=\infty$, general $1 \leq p<\infty$. Assume now that $\nu$ is infinite but finite on finite intervals. Then by a standard time change argument (see [48, Theorems 10.27 and 10.28] or [32, Subsection 5.5]) we can assume that a.s.

$$
\nu([s, t] \times J) \leq t-s, \quad 0 \leq s \leq t,
$$

which was considered in Step 2.

Step 4: $\nu$ is general, $1 \leq p<\infty$. If we have a general measure $\nu$, then we make the following two tricks. First, instead of considering $\mu$, we consider $\mu^{m}:=\left.\mu\right|_{A_{m}}$, where $\left(A_{m}\right)_{m \geq 1}$ is an increasing family of elements of $\widetilde{\mathcal{P}}$ such that $\cup_{m} A_{m}=\mathbb{R}_{+} \times \Omega \times J$ and $\mathbb{E} \mu\left(A_{m}\right)<\infty$ for any $m \geq 1$ (such a family exists as $\mu$ is $\widetilde{\mathcal{P}}$ - $\sigma$-finite). Note that by Step 3 for any $m \geq 1$ we have that

$$
\mathbb{E} \sup _{t \geq 0}\left\|\int_{[0, t] \times J} F \mathrm{~d} \bar{\mu}^{m}\right\|^{p} \bar{\sim}_{p, X} \mathbb{E}\left\|\int_{\mathbb{R}_{+} \times J} F \mathrm{~d} \bar{\mu}_{\text {Cox }}^{m}\right\|^{p} .
$$

Indeed, though $\mu^{m}$ is finite a.s., we can add to $\mu^{m}$ another independent Poisson random measure $\varepsilon \zeta, \varepsilon>0$, where $\zeta$ is a standard Poisson random measure with a compensator $\nu_{\zeta}$ satisfying $\nu_{\zeta}((s, t] \times\{j\})=t-s$ for all $0 \leq s \leq t$ and $j \in J$. Then by Step 3 we have that

$$
\mathbb{E} \sup _{t \geq 0}\left\|\int_{[0, t] \times J} F \mathrm{~d}\left(\bar{\mu}^{m}+\varepsilon \bar{\zeta}\right)\right\|^{p} \bar{\sim}_{p, X} \mathbb{E}\left\|\int_{\mathbb{R}_{+} \times J} F \mathrm{~d}\left(\bar{\mu}_{\text {Cox }}^{m}+\varepsilon \bar{\zeta}_{\text {Cox }}\right)\right\|^{p},
$$

and (3.37) follows by letting $\varepsilon \rightarrow 0$, by a triangle inequality, and by the fact that

$$
\mathbb{E} \sup _{t \geq 0}\left\|\int_{[0, t] \times J} F \mathrm{~d} \varepsilon \bar{\zeta}\right\|^{p} \bar{\sim}_{p, X} \mathbb{E}\left\|\int_{\mathbb{R}_{+} \times J} F \mathrm{~d} \varepsilon \bar{\zeta}_{\text {Cox }}\right\|^{p} \lesssim_{F, X, p} \varepsilon^{p} .
$$

Now notice that by Burkholder-Davis-Gundy inequalities [119, Subsection 7.2], by $\gamma$-dominated convergence [47, Theorem 9.4.2], and by monotone convergence theorem (see Subsection 2.11 for the definition of a $\gamma$-norm)

$$
\begin{aligned}
\mathbb{E} \sup _{t \geq 0}\left\|\int_{[0, t] \times J} F \mathrm{~d} \bar{\mu}^{m}-\int_{[0, t] \times J} F \mathrm{~d} \bar{\mu}\right\|^{p} & =\sup _{t \geq 0}\left\|\int_{[0, t] \times J} F \mathbf{1}_{\mathbb{R}_{+} \times \Omega \times J \backslash A_{m}} \mathrm{~d} \bar{\mu}\right\|^{p} \\
& \bar{\sim}_{p, X} \mathbb{E}\left\|F \mathbf{1}_{\mathbb{R}_{+} \times \Omega \times J \backslash A_{m}}\right\|_{\gamma\left(L^{2}\left(\mathbb{R}_{+} \times J ; \mu\right), X\right)}^{p}
\end{aligned}
$$




$$
\rightarrow 0, \quad m \rightarrow \infty
$$

(see also Section B), and for the same reason and the fact that we can set $\mu_{\mathrm{Cox}}^{m}$ to be $\left.\mu_{\mathrm{Cox}}\right|_{A_{m}}$ (as they are equidistributed)

$$
\mathbb{E}\left\|\int_{\mathbb{R}_{+} \times J} F \mathrm{~d} \bar{\mu}_{\text {Cox }}^{m}-\int_{\mathbb{R}_{+} \times J} F \mathrm{~d} \bar{\mu}_{\mathrm{Cox}}\right\|^{p} \rightarrow 0, \quad m \rightarrow \infty .
$$

Thus (3.10) follows as a limit of (3.37).

Remark 3.25. Note that $\int F \mathrm{~d} \bar{\mu}_{\text {Cox }}$ is a decoupled tangent martingale to $\int F \mathrm{~d} \bar{\mu}$. Indeed, let $(\Omega, \mathcal{F}, \mathbb{P})$ and $\mathbb{F}$ be the original probability space and filtration, let $(\bar{\Omega}, \overline{\mathcal{F}}, \overline{\mathbb{P}})$ be the extended by $\mu_{\mathrm{Cox}}$ probability space, and let $\overline{\mathbb{F}}=\left(\overline{\mathcal{F}}_{t}\right)_{t \geq 0}$ be such that $\overline{\mathcal{F}}_{t}:=\sigma\left(\mathcal{F}_{t},\left.\mu_{\mathrm{Cox}}\right|_{[0, t]}\right)$ for any $t \geq 0$. One needs to show that $M=\int F \mathrm{~d} \bar{\mu}$ is an $\overline{\mathbb{F}}$-martingale with the same local characteristics $\left(0, \nu^{M}\right)$ with $\nu^{M}$ defined by

$$
\nu^{M}(I \times B)=\int_{I} \mathbf{1}_{B \backslash\{0\}}(F) \mathrm{d} \nu, \quad I \subset \mathbb{R}_{+}, \quad B \subset X \text { Borel. }
$$

As $F$ is elementary predictable (but the same can be proven for any strongly $\widetilde{\mathcal{P}}$ measurable $F$ via exploiting an approximation argument, see Proposition 3.27 and Definition 3.28) and as $\mathcal{J}$ is countably generated, there exists an increasing family of finite $\sigma$-algebras $\left(\mathcal{J}^{m}\right)_{m \geq 1}$ on $J$ such that $F$ is $\mathcal{B}\left(\mathbb{R}_{+}\right) \otimes \mathcal{J}^{m} \otimes \mathcal{F}$ measurable for any $m \geq 1$ and $\mathcal{J}=\sigma\left(\left(\mathcal{J}^{m}\right)_{m \geq 1}\right)$. Let $\overline{\mathbb{F}}^{m}=\left(\overline{\mathcal{F}}_{t}^{m}\right)_{t \geq 0}$ be such that $\overline{\mathcal{F}}_{t}^{m}:=\sigma\left(\mathcal{F}_{t},\left.\mu_{\mathrm{Cox}}\right|_{[0, t] \times \mathcal{J}^{m}}\right)$ for any $t \geq 0$, where $\sigma\left(\left.\mu_{\mathrm{Cox}}\right|_{[0, t] \times \mathcal{J}^{m}}\right)$ means that we are considering $\sigma$-algebras generated by processes $t \mapsto \mu_{\mathrm{Cox}}([0, t] \times A)$ for all $A \in \mathcal{J}^{m}$ (by approximating $F$ as it was done in Step 4 of the proof of Theorem 3.22 we may assume that $\mathbb{E} \mu_{\mathrm{Cox}}\left(\mathbb{R}_{+} \times J\right)=\mathbb{E} \nu\left(\mathbb{R}_{+} \times J\right)=\mathbb{E} \mu\left(\mathbb{R}_{+} \times\right.$ $J)<\infty)$, and let us first show that $M$ is an $\overline{\mathbb{F}}^{m}$-martingale. Let $\left(A_{n}^{m}\right)_{n=1}^{N_{m}} \subset J$ be the partition generating $\mathcal{J}^{m}$ and let $\tau_{s}^{n}=\inf \left\{t \geq 0: \nu\left([0, t] \times A_{n}^{m}\right) \geq s\right\}$. Then thanks to Example 3.21 and the fact that the distribution of a Cox process is uniquely determined by its compensator we have that there exist independent standard Poisson processes $\left(N^{n}\right)_{n=1}^{N_{m}}$ which are also independent of $\mathcal{F}$ such that $\mu_{\text {Cox }}\left(\left[0, \tau_{s}^{n}\right] \times A\right)=N_{s}^{n}$. Fix any $t \geq r \geq 0$. Let $\mathcal{N}^{m}:=\sigma\left(\left(N^{n}\right)_{n=1}^{N_{m}}\right)$. Then

$$
\mathbb{E}\left(M_{t}-M_{r} \mid \overline{\mathcal{F}}_{r}^{m}\right) \stackrel{(*)}{=} \mathbb{E}\left(\mathbb{E}\left(M_{t}-M_{r} \mid \mathcal{F}_{r} \otimes \mathcal{N}^{m}\right) \mid \overline{\mathcal{F}}_{r}^{m}\right) \stackrel{(* *)}{=} 0
$$

where (*) follows from the fact that $\overline{\mathcal{F}}_{r}^{m} \subset \mathcal{F}_{r} \otimes \mathcal{N}^{m}$, and (**) holds as $M_{t}-M_{r}$ is independent of $\mathcal{N}^{m}$ and as $\mathbb{E}\left(M_{t}-M_{r} \mid \mathcal{F}_{r}\right)=0$. Now it suffices to notice that $\mathbb{E}\left(M_{t}-M_{r} \mid \overline{\mathcal{F}}_{r}\right)=\lim _{m \rightarrow \infty} \mathbb{E}\left(M_{t}-M_{r} \mid \overline{\mathcal{F}}_{r}^{m}\right)=0$ due to the martingale convergence theorem [46, Theorem 3.3.2]. The fact that $M$ keeps the same local characteristics $\left(0, \nu^{M}\right)$ can be shown by (3.38) via proving that $\mu$ has the same compensator after enlarging the probability space and filtration. Assume that $\mu$ has a different $\overline{\mathbb{F}}$-compensator $\tilde{\nu}$. Then for any $I \subset \mathbb{R}_{+}$and $A \subset J$ we have that $t \mapsto \mu([0, t] \cap I \times J)-\nu([0, t] \cap I \times J)$ is a martingale (as an integral w.r.t. $\bar{\mu}$ by the first part of the remark) and $t \mapsto \mu([0, t] \cap I \times J)-\tilde{\nu}([0, t] \cap I \times J)$ is a martingale (by the definition of a compensator), so a predictable finite variation 
process $t \mapsto \nu([0, t] \cap I \times J)-\tilde{\nu}([0, t] \cap I \times J)$ is a martingale thus it is zero by [56, Lemma 25.11] and hence $\tilde{\nu}=\nu$ a.s.

Remark 3.26. Inequality (3.10) has an equivalent formulation in terms of Poisson random measures. Indeed, as $\nu$ is $\widetilde{P}-\sigma$-finite, it is a.s. $\sigma$-finite, so by Subsection 2.9 a.s. there exists a Poisson random measure $N_{\nu}$, which distribution by the definition coincides with the distribution of the Cox process directed by $\nu$, so we have that for a.e. $\omega \in \Omega$ the distributions of $\int_{\mathbb{R}_{+} \times J} F \mathrm{~d} \bar{\mu}_{\text {Cox }}$ and $\int_{\mathbb{R}_{+} \times J} F \mathrm{~d} \tilde{N}_{\nu}$ coincide, and in particular by (3.10)

$$
\mathbb{E} \sup _{t \geq 0}\left\|\int_{[0, t] \times J} F \mathrm{~d} \bar{\mu}\right\|^{p} \bar{\sim}_{p, X} \mathbb{E}\left\|\int_{\mathbb{R}_{+} \times J} F \mathrm{~d} \widetilde{N}_{\nu}\right\|^{p}
$$

where the right-hand side is very much in the spirit of $\gamma$-radonifying operators (see Subsection 2.11; see also [3, 98]), but here instead of considering Gaussian random variables we have Poisson random measures. Note that this parallel with $\gamma$-radonifying operators might mislead as e.g. one has that $\left\|\int_{\mathbb{R}_{+} \times J} F \mathrm{~d} \widetilde{N}_{\nu}\right\|_{L^{p}(\Omega ; X)}$ and $\left\|\int_{\mathbb{R}_{+} \times J} F \mathrm{~d} \widetilde{N}_{\nu}\right\|_{L^{q}(\Omega ; X)}$ are incomparable for different $p$ and $q$ (a classical example would be $\alpha$-stable processes which can be represented as such integrals) which is of course not the case for Wiener integrals, see e.g. [47, Proposition 6.3.1].

Assume that for a Banach space $X$, for some $1 \leq p<\infty$, and for any Poisson random measure $N$ on $\mathbb{R}_{+} \times J$ with a compensator $\nu$ there exists a function $R_{\nu}$ acting on deterministic $X$-valued functions on $\mathbb{R}_{+} \times J$ such that $\mathbb{E}\left\|\int_{\mathbb{R}_{+} \times J} F \mathrm{~d} \tilde{N}\right\|^{p} \lesssim_{p, X} R_{\nu}(F)$ (resp. $\mathbb{E}\left\|\int_{\mathbb{R}_{+} \times J} F \mathrm{~d} \tilde{N}\right\|^{p} \gtrsim_{p, X} R_{\nu}(F)$.) In this case, if $X$ is a UMD Banach space, we can conclude from (3.40) that

$$
\begin{aligned}
& \mathbb{E} \sup _{t \geq 0}\left\|\int_{[0, t] \times J} F \mathrm{~d} \bar{\mu}\right\|^{p} \lesssim_{p, X} \mathbb{E} R_{\nu}(F) \\
& \quad\left(\text { resp. } \mathbb{E} \sup _{t \geq 0}\left\|\int_{[0, t] \times J} F \mathrm{~d} \bar{\mu}\right\|^{p} \gtrsim_{p, X} \mathbb{E} R_{\nu}(F)\right) .
\end{aligned}
$$

An example of such $R_{\nu}$ in the case of martingale type $r$ spaces was presented in [43, 121]:

$$
R_{\nu}(F):=\left\{\begin{array}{l}
\min \left\{\int_{\mathbb{R}_{+} \times J}\|F\|^{p} \mathrm{~d} \nu,\left(\int_{\mathbb{R}_{+} \times J}\|F\|^{r} \mathrm{~d} \nu\right)^{\frac{p}{r}}\right\}, \quad 1 \leq p \leq r, \\
\int_{\mathbb{R}_{+} \times J}\|F\|^{p} \mathrm{~d} \nu+\left(\int_{\mathbb{R}_{+} \times J}\|F\|^{r} \mathrm{~d} \nu\right)^{\frac{p}{r}}, \quad p>r
\end{array}\right.
$$

(though [43, 121] work only with Poisson random measures with the compensator of the form $\lambda_{\mathbb{R}_{+}} \otimes \nu_{0}$, in the case of deterministic $F$ these estimates can be generalized to a general compensated Poisson measure). Therefore (3.40) allows us to extend [43, 121] to stochastic integrals with respect to general random measures for martingale type $r$ Banach spaces with the UMD property. 


\subsection{Purely discontinuous quasi-left continuous martingales}

The present subsection is devoted to the purely discontinuous quasi-left continuous case. Our goal is to show that any $X$-valued purely discontinuous quasi-left continuous martingale $M$ coincides with $\int x \mathrm{~d} \bar{\mu}^{M}(\cdot, x)$ (where $\mu^{M}$ is defined by (2.14)), so that we can reduce this case to the one considered in Subsection 3.4. To this end we need to define an integral of a general predictable (i.e. not necessarily elementary predictable) process with respect to a random measure (as $(t, x) \mapsto x, t \geq 0, x \in X$ is not elementary predictable). Let us start with the following proposition.

Proposition 3.27. Let $(J, \mathcal{J})$ be a measurable space, $\mu$ be an integer-valued optional random measure over $\mathbb{R}_{+} \times J$, $\nu$ be its compensator, $\bar{\mu}:=\mu-\nu$. Let $X$ be a Banach space. Let $F: \mathbb{R}_{+} \times \Omega \times J \rightarrow X$ be strongly $\widetilde{\mathcal{P}}$-measurable so that $\mathbb{E} \int_{\mathbb{R}_{+} \times J}\|F\| \mathrm{d} \mu<\infty$. Then

$$
M_{t}:=\int_{[0, t] \times J} F \mathrm{~d} \bar{\mu}=\int_{[0, t] \times J} F \mathrm{~d} \mu-\int_{[0, t] \times J} F \mathrm{~d} \nu, \quad t \geq 0,
$$

is well defined and is a martingale. Moreover,

$$
\mathbb{E} \sup _{t \geq 0}\left\|M_{t}\right\| \leq 2 \mathbb{E} \int_{\mathbb{R}_{+} \times J}\|F\| \mathrm{d} \mu
$$

Proof. First of all note that $M$ is well defined by formula (3.41) as by Fubini's theorem $F$ is a.s. $\mathcal{B}\left(\mathbb{R}_{+}\right) \otimes \mathcal{J}$-measurable and a.s. integrable with respect to $\mu$ and $\nu$ (the a.s. integrability w.r.t. $\nu$ holds since $\mathbb{E} \int_{\mathbb{R}_{+} \times J}\|F\| \mathrm{d} \nu=\mathbb{E} \int_{\mathbb{R}_{+} \times J}\|F\| \mathrm{d} \mu<$ $\infty$, see (2.11)). Also notice that (3.42) follows directly from (3.41), from a triangle inequality, and from (2.11).

As $F$ is strongly $\widetilde{\mathcal{P}}$-measurable, as $F \in L^{1}\left(\Omega \times \mathbb{R}_{+} \times J, \mathbb{P} \otimes \nu ; X\right)$, and as step functions are dense in $L^{1}\left(\Omega \times \mathbb{R}_{+} \times J, \widetilde{\mathcal{P}}, \mathbb{P} \otimes \nu ; X\right)$ (here we choose the measure $\nu$ so that $\mathbb{P} \otimes \nu$ is a measure on $\widetilde{\mathcal{P}})$, there exist elementary predictable processes $\left(F^{n}\right)_{n \geq 1}, F^{n}: \mathbb{R}_{+} \times \Omega \times J \rightarrow X$ for any $n \geq 1$, such that

$$
\mathbb{E} \int_{\mathbb{R}_{+} \times J}\left\|F^{n}\right\| \mathrm{d} \mu=\mathbb{E} \int_{\mathbb{R}_{+} \times J}\left\|F^{n}\right\| \mathrm{d} \nu<\infty
$$

for any $n \geq 1$ and

$$
\mathbb{E} \int_{\mathbb{R}_{+} \times J}\left\|F-F^{n}\right\| \mathrm{d} \mu=\mathbb{E} \int_{\mathbb{R}_{+} \times J}\left\|F-F^{n}\right\| \mathrm{d} \nu \rightarrow 0, \quad n \rightarrow \infty .
$$

For each $n \geq 1$ let

$$
M_{t}^{n}:=\int_{[0, t] \times J} F^{n} \mathrm{~d} \bar{\mu}=\int_{[0, t] \times J} F^{n} \mathrm{~d} \mu-\int_{[0, t] \times J} F^{n} \mathrm{~d} \nu, \quad t \geq 0 .
$$


Then $M^{n}$ is a martingale. On the other hand by (3.42) we have that

$$
\mathbb{E} \sup _{t \geq 0}\left\|M_{t}-M_{t}^{n}\right\| \leq 2 \mathbb{E} \int_{\mathbb{R}_{+} \times J}\left\|F-F^{n}\right\| \mathrm{d} \mu \rightarrow 0, \quad n \rightarrow \infty,
$$

and thus, as martingales form a closed subset of $L^{1}\left(\Omega ; \mathcal{D}\left(\mathbb{R}_{+}, X\right)\right)$ (see Definition 2.2 and Theorem 2.9), $M$ is a martingale as well.

Now we are ready to define an integral of a general process with respect to a random measure.

Definition 3.28. Let $(J, \mathcal{J})$ be a measurable space, $\mu$ be an integer-valued optional random measure on $\mathbb{R}_{+} \times J, \nu$ be its compensator, $\bar{\mu}:=\mu-\nu$. Let $X$ be a Banach space. A general strongly $\widetilde{\mathcal{P}}$-measurable process $F: \mathbb{R}_{+} \times \Omega \times J \rightarrow X$ is called to be integrable with respect to $\bar{\mu}$ if for any increasing family $\left(A_{n}\right)_{n \geq 1}$ of elements of $\widetilde{\mathcal{P}}$ satisfying $\mathbb{E} \int_{A_{n}}\|F\| \mathrm{d} \mu<\infty$ for any $n \geq 1$ and $\cup_{n \geq 1} A_{n}=$ $\mathbb{R}_{+} \times \Omega \times J$, we have that the processes $\int_{A_{n}} F \mathrm{~d} \bar{\mu}$ converge in $L^{1}\left(\Omega ; \mathcal{D}\left(\mathbb{R}_{+}, X\right)\right)$ as $n \rightarrow \infty$.

$F$ is called to be locally integrable with respect to $\bar{\mu}$ if there exists an increasing sequence of stopping times $\left(\tau_{n}\right)_{n \geq 1}$ such that $\tau_{n} \rightarrow \infty$ as $n \rightarrow \infty$ and $A \mathbf{1}_{\left[0, \tau_{n}\right]}$ is integrable with respect to $\bar{\mu}$ for any $n \geq 1$.

This definition is very much in the spirit of Lebesgue integration (a function $f$ can be integrable only if its restrictions $\left.f\right|_{B_{n}}$ are integrable and the corresponding integrals converge as the restriction domains $B_{n}$ 's blow up) or vector-valued stochastic integration with respect to a Brownian motion, see [81].

Remark 3.29. Notice that in this case

$$
t \mapsto \int_{A_{n} \cap[0, t] \times J} F \mathrm{~d} \bar{\mu}=\int_{A_{n} \cap[0, t] \times J} F \mathrm{~d} \mu-\int_{A_{n} \cap[0, t] \times J} F \mathrm{~d} \nu, \quad t \geq 0,
$$

are well defined martingales by Proposition 3.2\%.

Now let us formulate the main theorem of the present subsection.

Theorem 3.30. Let $X$ be a Banach space. Then $X$ is UMD if and only if for any purely discontinuous quasi-left continuous martingale $M: \mathbb{R}_{+} \times \Omega \rightarrow X$ with $\mathbb{E} \sup _{t \geq 0}\left\|M_{t}\right\|<\infty$ we have that $x$ is integrable with respect to $\bar{\mu}^{M}$. If this is the case, then $M_{t}=\int_{[0, t] \times X} x \mathrm{~d} \bar{\mu}^{M}$ a.s.

Proof. We will separately prove the "if" and the "only if" parts.

The "only if" part. Let $X$ be a UMD Banach space, $M: \mathbb{R}_{+} \times \Omega \rightarrow X$ be a purely discontinuous quasi-left continuous martingale with $\mathbb{E} \sup _{t \geq 0}\left\|M_{t}\right\|<\infty$, and let $\left(A_{n}\right)_{n \geq 1}$ be some increasing family from $\widetilde{\mathcal{P}}$ satisfying the properties from Definition 3.28. For every $n \geq 1$ define an $X$-valued martingale

$$
M_{t}^{n}:=\int_{A_{n} \cap[0, t] \times X} x \mathrm{~d} \bar{\mu}^{M}, \quad t \geq 0 .
$$


We need to show that $\left(M^{n}\right)_{n \geq 1}$ converges in $L^{1}\left(\Omega ; \mathcal{D}\left(\mathbb{R}_{+}, X\right)\right)$ as $n \rightarrow \infty$. Note that by the definition of $M^{n}$ we have that $\Delta M_{t}^{n}(\omega)=\Delta M_{t} \mathbf{1}_{A_{n}}\left(t, \omega, \Delta M_{t}(\omega)\right)$ for a.e. $\omega \in \Omega$ for any $t \geq 0$, and thus, as $M$ and $M^{n}$ are purely discontinuous $\left(M^{n}\right.$ is purely discontinuous as it is an integral with respect to a random measure, see e.g. [52, §II.1d] or $[116,118])$, by $[119$, Subsection 6.1$]$ we have that (see Subsection 2.11 for the definition of a $\gamma$-norm)

$$
\infty>\mathbb{E} \sup _{t \geq 0}\left\|M_{t}\right\| \bar{\sim}_{X} \mathbb{E}\left\|\left(\Delta M_{t}\right)_{t \geq 0}\right\|_{\gamma\left(\ell^{2}\left(\mathbb{R}_{+}\right), X\right)}
$$

also note that for any $n \geq 1$ by Remark 3.29 we have that $\mathbb{E} \sup _{t \geq 0}\left\|M_{t}^{n}\right\|<\infty$. Thus we have that

$$
\mathbb{E} \sup _{t \geq 0}\left\|M_{t}-M_{t}^{n}\right\| \bar{\sim}_{X} \mathbb{E}\left\|\left(\Delta M_{t} \mathbf{1}_{\bar{A}_{n}}\left(t, \cdot, \Delta M_{t}(\cdot)\right)\right)_{t \geq 0}\right\|_{\gamma\left(\ell^{2}\left(\mathbb{R}_{+}\right), X\right)},
$$

(here $\bar{A}_{n}$ means the complement of $A$ in $\left.\mathbb{R}_{+} \times \Omega \times J\right)$. As $\left(\bar{A}_{n}\right)_{n \geq 1}$ is a vanishing family, by $\gamma$-dominated convergence [47, Theorem 9.4.2] and by the monotone convergence theorem we have that $\mathbb{E} \sup _{t>0}\left\|M_{t}-M_{t}^{n}\right\| \rightarrow 0$ as $n \rightarrow \infty$. Consequently, $M^{n}$ converges to $M$ in $L^{1}\left(\Omega ; \mathcal{D}\left(\overline{\mathbb{R}}_{+}, X\right)\right)$.

The "if" part. This part of the proof is based on the tricks from [118, Subsection 4.4]. Assume that $X$ is not UMD. Our goal is to find such a purely discontinuous quasi-left continuous martingale $M$ and such an increasing family of sets $\left(A_{n}\right)_{n \geq 1}$ in $\widetilde{\mathcal{P}}$ that $\int_{A_{n}}\|x\| \mathrm{d} \mu^{M}<\infty$ for any $n \geq 1$, but $\int x \mathbf{1}_{A_{n}} \mathrm{~d} \bar{\mu}^{M}$ diverges in $L^{1}\left(\Omega ; \mathcal{D}\left(\mathbb{R}_{+}, X\right)\right)$.

Due to the formula $[15,(1.7)]$ and $[118$, Subsection 4.4] we have that $X$ is UMD if and only if

$$
\mathbb{E} \sup _{n}\left\|g_{n}\right\| \lesssim X \mathbb{E} \sup _{n}\left\|f_{n}\right\|
$$

for any $X$-valued discrete martingales $f=\left(f_{n}\right)_{n \geq 0}$ and $g=\left(g_{n}\right)_{n \geq 0}$ with $g_{0}=$ $f_{0}=0$ and with

$$
g_{n}-g_{n-1}=\varepsilon_{n}\left(f_{n}-f_{n-1}\right), \quad n \geq 1,
$$

for any fixed $\{0,1\}$-valued sequence $\left(\varepsilon_{n}\right)_{n \geq 1}$. As $X$ is not UMD, (3.44) does not hold. Therefore there exists a Paley-Walsh martingale $\left(f_{n}\right)_{n \geq 1}$ (see $[18,46]$ why we can restrict to the Paley-Walsh case), i.e. a martingale $\left(f_{n}\right)_{n \geq 0}$ such that there exists a sequence $\left(r_{n}\right)_{n \geq 1}$ of Rademachers (see Definition 2.1) so that $f_{n}-f_{n-1}=r_{n} \phi_{n}\left(r_{1}, \ldots, r_{n}\right)$ for some $\phi_{n}:\{-1,1\}^{n-1} \rightarrow X$ for every $n \geq 1$ and $f_{0}=0$, and a $\{0,1\}$-valued sequence $\left(\varepsilon_{n}\right)_{n \geq 1}$, such that we have that $\mathbb{E} \sup _{n}\left\|f_{n}\right\|=1$ and $\mathbb{E} \sup _{n}\left\|g_{n}\right\|=\infty$ for $\left(g_{n}\right)_{n \geq 0}$ satisfying (3.45) and $g_{0}=0$.

Let $N^{1}$ and $N^{2}$ be two independent standard Poisson processes (Note that in this case $N^{1}-N^{2}$ has a zero compensator and thus it is a martingale). Let $\tau_{0}=0$, and for each $n \geq 1$ define

$$
\tau_{n}:=\inf \left\{t \geq \tau_{n-1}:\left(N_{t}^{1}-N_{\tau_{n-1}}^{1}\right) \vee\left(N_{t}^{2}-N_{\tau_{n-1}}^{2}\right) \neq 0\right\}, \quad n \geq 1 .
$$

Note that $\tau_{n}<\infty$ a.s. and that $\tau_{n} \rightarrow \infty$ a.s. as $n \rightarrow \infty$ (for the construction of the standard Poisson process we refer the reader e.g. to $[56,61,102,103]$ or in 
any other standard probability textbook), and that as Poisson processes have strong Markov property,

$$
\left(\sigma_{n}\right)_{n \geq 1}=\left(N_{\tau_{n}}^{1}-N_{\tau_{n}}^{2}-\left(N_{\tau_{n-1}}^{1}-N_{\tau_{n-1}}^{2}\right)\right)_{n \geq 1}
$$

are i.i.d. random variables. Moreover, as $N^{1}$ and $N^{2}$ can have jumps of size 1 a.s., $\sigma_{n} \in\{-1,1\}$ a.s., and as $N^{1}$ and $N^{2}$ are independent equidistributed, $\left(\sigma_{n}\right)_{n \geq 1}$ are independent Rademachers. In particular, for a simplicity of the proof we identify $\left(\sigma_{n}\right)_{n \geq 1}$ with $\left(r_{n}\right)_{n \geq 1}$.

Now let us consider a martingale $M: \mathbb{R}_{+} \times \Omega \rightarrow X$ of the form $M=$ $\int \Phi \mathrm{d}\left(N^{1}-N^{2}\right)$, where

$$
\Phi(t):=\sum_{n=1}^{\infty} \phi_{n}\left(r_{1}, \ldots, r_{n}\right) \mathbf{1}_{\left(\tau_{n-1}, \tau_{n}\right]}(t), \quad t \geq 0,
$$

and the integral is defined in the Riemann-Stieltjes way $\left(N^{1}-N^{2}\right.$ is a.s. locally of finite variation). First of all, $M$ is a local martingale since for any $n \geq 1$ we have that $\Phi^{\tau_{n}}$ is bounded and takes values in a finite-dimensional subspace of $X$, so the stochastic integral $M^{\tau_{n}}=\int \Phi^{\tau_{n}} \mathrm{~d}\left(N^{1}-N^{2}\right)$ is well defined by a classical finite dimensional theory (see $[52,56]$ ). Moreover, as $M_{\tau_{n}}=f_{n}$ a.s. and as $M$ is a.s. a constant on $\left[\tau_{n-1}, \tau_{n}\right)$ for any $n \geq 1, \mathbb{E} M^{*}=\mathbb{E} \sup _{n}\left\|f_{n}\right\|=1$, and thus by the dominated convergence theorem and by the fact that a conditional expectation is a contraction on $L^{1}(\Omega ; X)$ (see [46, Section 2.6])

$$
\mathbb{E}\left(M_{t} \mid \mathcal{F}_{s}\right)=\lim _{n \rightarrow \infty} \mathbb{E}\left(M_{\tau_{n} \wedge t} \mid \mathcal{F}_{s}\right)=\lim _{n \rightarrow \infty} M_{\tau_{n} \wedge s}=M_{s}, \quad 0 \leq s \leq t,
$$

so $M$ is a martingale with $\mathbb{E} M^{*}<\infty$.

Since $\mathbb{E} \sup _{n}\left\|g_{n}\right\|=\infty$, there exists a sequence $0=k_{1}<\ldots<k_{m}<\ldots$ such that $\mathbb{E} \sup _{k_{m}<n \leq k_{m+1}}\left\|g_{n}-g_{k_{m}}\right\|>1$ for each $m \geq 1$. Set $J=X$. Define $\mathbb{R}_{+} \times \Omega \times J \supset A_{0}=\varnothing$ and

$$
\begin{aligned}
A_{2 m-1}:=A_{2 m-2} \cup_{k_{m}<n \leq k_{m+1}, \varepsilon_{n}=1}\left(\tau_{n-1}, \tau_{n}\right] & \in \widetilde{\mathcal{P}}, \quad m \geq 1, \\
A_{2 m}:=A_{2 m-1} \cup_{k_{m}<n \leq k_{m+1}, \varepsilon_{n}=0}\left(\tau_{n-1}, \tau_{n}\right] & \in \widetilde{\mathcal{P}}, \quad m \geq 1 .
\end{aligned}
$$

Then we have that $\cup_{m} A_{m}=\mathbb{R}_{+} \times \Omega \times J$ and that $\left(A_{m}\right)_{m \geq 1}$ in increasing. Let us first show that $\mathbb{E} \int_{A_{m}}\|x\| \mathrm{d} \mu^{M}<\infty$ for any $m \geq 1$

$$
\begin{aligned}
& \mathbb{E} \int_{A_{m}}\|x\| \mathrm{d} \mu^{M} \leq \mathbb{E} \int_{\left[0, \tau_{k_{m+1}}\right] \times J}\|x\| \mathrm{d} \mu^{M} \stackrel{(*)}{=} \mathbb{E} \sum_{0 \leq s \leq \tau_{k_{m+1}}}\left\|\Delta M_{s}\right\| \\
& \stackrel{(* *)}{=} \mathbb{E} \sum_{n=1}^{k_{m+1}}\left\|\Delta M_{\tau_{n}}\right\|=\mathbb{E} \sum_{n=1}^{k_{m+1}}\left\|f_{n}-f_{n-1}\right\| \leq 2 k_{m+1} \mathbb{E} \sup _{n}\left\|f_{n}\right\| \leq 2 k_{m+1},
\end{aligned}
$$

where $(*)$ follows from the definition $(2.14)$ of $\mu^{M}$ and (**) follows from the fact that $M$ is a.s. a constant on $\left[\tau_{n-1}, \tau_{n}\right)$ for any $n \geq 1$ and from the definition of 
$M$. Therefore $x \mathbf{1}_{A_{m}}$ is integrable with respect to $\bar{\mu}^{M}$ by Proposition 3.27. Let us now show that $\int_{A_{m}} x \mathrm{~d} \bar{\mu}^{M}$ does not converge in $L^{1}\left(\Omega ; \mathcal{D}\left(\mathbb{R}_{+}, X\right)\right)$. It is sufficient to show that $\int_{A_{2 m-1}} x \mathrm{~d} \bar{\mu}^{M}-\int_{A_{2 m-2}} x \mathrm{~d} \bar{\mu}^{M}$ is big enough for any $m \geq 1$ :

$$
\begin{array}{rl}
\mathbb{E} \sup _{t \geq 0} \| \int_{A_{2 m-1} \cap[0, t] \times J} x & \mathrm{~d} \bar{\mu}^{M}-\int_{A_{2 m-2} \cap[0, t] \times J} x \mathrm{~d} \bar{\mu}^{M} \| \\
& =\mathbb{E} \sup _{t \geq 0}\left\|\int_{A_{2 m-1} \backslash A_{2 m-2} \cap[0, t] \times J} x \mathrm{~d} \bar{\mu}^{M}\right\| \\
& \stackrel{(i)}{=} \mathbb{E} \sup _{t \geq 0}\left\|\sum_{n=k_{m}+1}^{k_{m+1}} \mathbf{1}_{\varepsilon_{n}=1}\left(M_{\tau_{n} \wedge t}-M_{\tau_{n-1} \wedge t}\right)\right\| \\
& \stackrel{(i i)}{=} \mathbb{E} \underset{n=k_{m}+1}{k_{m+1}}\left\|\sum_{k=k_{m}+1}^{n} \mathbf{1}_{\varepsilon_{n}=1}\left(M_{\tau_{n}}-M_{\tau_{n-1}}\right)\right\| \\
& \stackrel{(i i i)}{=} \mathbb{E} \underset{\substack{k_{m+1} \\
n=k_{m}+1}}{\sup }\left\|g_{n}-g_{k_{m}}\right\| \geq 1,
\end{array}
$$

where in (i) we used the definition (2.14) of $\mu^{M}$, the fact that $M$ is pure jump, and (3.46), (ii) follows from the fact that $M$ is pure jump and has its jumps at $\left\{\tau_{1}, \ldots, \tau_{n}, \ldots\right\}$, and (iii) follows from the definition of $M$ and $g$. Thus $x$ is not integrable with respect to $\bar{\mu}^{M}$.

Remark 3.31. Though in the sequel we will need only the "only if" part of the theorem above, the author decided to include the "if" statement with such a complicated proof as well because he found such a nontrivial characterization of the UMD property rather important and elegant.

Remark 3.32. Note that under the so-called Radon-Nikodým property (many spaces have this property, e.g. reflexive spaces, see [46, Definition 1.3.9] and the references therein) in Theorem 3.30 there is no difference between considering martingales over $\mathbb{R}_{+}$and over $[0,1]$. Indeed, if $M: \mathbb{R}_{+} \times \Omega \rightarrow X$ is such that $\mathbb{E} \sup _{t>0}\left\|M_{t}\right\|<\infty$ and if $X$ has the Radon-Nikodým property, then by [46, Theorem 3.3.16] $M$ has an $L^{1}$-limit $M_{\infty}$. Therefore we can define a time-change $t \mapsto 2 \arctan t / \pi$, a time-changed filtration $\mathbb{G}=\left(\mathcal{G}_{t}\right)_{t \geq 0}$ with $\mathcal{G}_{t}=\mathcal{F}_{\tan (\pi t / 2)}$ for $0 \leq t<1$ and $\mathcal{G}_{t}=\mathcal{F}_{\infty}$ for $t \geq 1$, and a $\mathbb{G}$-martingale $\widetilde{M}$ with $\widetilde{M}_{t}=M_{\tan (\pi t / 2)}$ for $0 \leq t<1$ and $\widetilde{M}=M_{\infty}$ for $t \geq 1$. In this case we have that $x$ in integrable with respect to $\bar{\mu}^{M}$ if and only if it is integrable with respect $\bar{\mu}^{\widetilde{M}}$.

Let us now state the corollaries we were looking for.

Corollary 3.33. Let $X$ be a Banach space, $1 \leq p<\infty$. Then $X$ has the UMD property if and only if for any pair $M, N: \mathbb{R}_{+} \times \Omega \rightarrow X$ of tangent purely discontinuous quasi-left continuous martingales one has that

$$
\mathbb{E} \sup _{t \geq 0}\left\|M_{t}\right\|^{p} \bar{\sim}_{p, X} \mathbb{E} \sup _{t \geq 0}\left\|N_{t}\right\|^{p} .
$$

For the proof we will need the following lemma. 
Lemma 3.34. Let $X$ be a UMD Banach space, $(J, \mathcal{J})$ be a measurable space, $\mu^{1}$ and $\mu^{2}$ be optional quasi-left continuous random measures on $\mathbb{R}_{+} \times J$. Assume that $\mu^{1}$ and $\mu^{2}$ have the same compensator $\nu$. Let $\bar{\mu}^{1}:=\mu^{1}-\nu$ and $\bar{\mu}^{2}:=\mu^{2}-\nu$. Let $F: \mathbb{R}_{+} \times \Omega \rightarrow X$ be a strongly $\mathcal{P}$-measurable process. Then $F$ is stochastically integrable with respect to $\bar{\mu}^{1}$ if and only if it is stochastically integrable with respect to $\bar{\mu}^{2}$. Moreover, if this is the case, then $\int F \mathrm{~d} \bar{\mu}^{1}$ and $\int F \mathrm{~d} \bar{\mu}^{2}$ are tangent.

Proof. Let us first show the "if and only if" statement. Recall that stochastic integrability of a general $X$-valued predictable process with respect to a random measure was defined in Definition 3.28. Assume that $F$ is stochastically integrable with respect to $\bar{\mu}^{1}$. Let us show that $F$ is stochastically integrable with respect to $\bar{\mu}^{2}$. Let $\left(A_{n}\right)_{n \geq 1}$ be an increasing sequence of sets in $\widetilde{\mathcal{P}}$ with $\cup_{n} A_{n}=\Omega \times \mathbb{R}_{+} \times J$ satisfying $\mathbb{E} \int_{A_{n}}\|F\| \mathrm{d} \mu^{1}<\infty$ for any $n \geq 1$. Then by (2.11) we have that

$$
\mathbb{E} \int_{A_{n}}\|F\| \mathrm{d} \mu^{2}=\mathbb{E} \int_{A_{n}}\|F\| \mathrm{d} \nu=\mathbb{E} \int_{A_{n}}\|F\| \mathrm{d} \mu^{1}<\infty, \quad n \geq 1,
$$

so $F \mathbf{1}_{A_{n}}$ is stochastically integrable with respect to $\bar{\mu}^{1}$ and $\bar{\mu}^{2}$ by Proposition 3.27. For each $n \geq 1$ let us set

$$
M_{t}^{n}:=\int_{[0, t] \times J} F \mathbf{1}_{A_{n}} \mathrm{~d} \bar{\mu}^{1}, \quad N_{t}^{n}:=\int_{[0, t] \times J} F \mathbf{1}_{A_{n}} \mathrm{~d} \bar{\mu}^{2}, \quad t \geq 0 .
$$

As $F$ is stochastically integrable with respect to $\bar{\mu}^{1}, M^{n}$ is a Cauchy sequence in $L^{1}\left(\Omega ; \mathcal{D}\left(\mathbb{R}_{+}, X\right)\right)$. By Theorem 3.22 and by the fact that $\mu^{1}$ and $\mu^{2}$ have the same compensator we have that for any $m \geq n \geq 1$

$$
\begin{aligned}
\mathbb{E} \sup _{t \geq 0}\left\|N_{t}^{m}-N_{t}^{n}\right\| & =\mathbb{E} \sup _{t \geq 0}\left\|\int_{[0, t] \times J} F \mathbf{1}_{A_{m} \backslash A_{n}} \mathrm{~d} \bar{\mu}^{2}\right\| \\
& \bar{\sim}_{X} \mathbb{E} \sup _{t \geq 0}\left\|\int_{[0, t] \times J} F \mathbf{1}_{A_{m} \backslash A_{n}} \mathrm{~d} \bar{\mu}^{1}\right\|=\mathbb{E} \sup _{t \geq 0}\left\|M_{t}^{m}-M_{t}^{n}\right\|,
\end{aligned}
$$

so $N^{n}$ is a Cauchy sequence in $L^{1}\left(\Omega ; \mathcal{D}\left(\mathbb{R}_{+}, X\right)\right)$, and thus $F$ is integrable with respect to $\bar{\mu}^{2}$ by Definition 3.28.

Let us show that $M:=\int F \mathrm{~d} \bar{\mu}^{1}$ and $N:=\int F \mathrm{~d} \bar{\mu}^{2}$ are tangent, i.e. as $M$ and $N$ are purely discontinuous, we need to show that the compensators $\nu^{M}$ and $\nu^{N}$ of $\mu^{M}$ and $\mu^{N}$ respectively coincide. Fix a predictable set $A \subset \mathbb{R}_{+} \times \Omega \times X$. Then for any $t \geq 0$ we have that a.s.

$$
\int_{[0, t] \times X} \mathbf{1}_{A} \mathrm{~d} \mu^{M}=\sum_{0 \leq s \leq t} \mathbf{1}_{A}\left(s, \cdot, \Delta M_{s}\right)=\int_{[0, t] \times J} \mathbf{1}_{A}(\cdot, \cdot, F) \mathrm{d} \mu^{1}
$$

(the latter can be infinite), so by the definition of a compensator we have that

$$
\mathbb{E} \int_{\mathbb{R}_{+} \times X} \mathbf{1}_{A} \mathrm{~d} \nu^{M}=\mathbb{E} \int_{\mathbb{R}_{+} \times J} \mathbf{1}_{A}(\cdot, \cdot, F) \mathrm{d} \nu .
$$

The same can be shown for $\nu^{N}$. Therefore, as $A$ was arbitrary predictable, $\nu^{M}$ and $\nu^{N}$ coincide, so $M$ and $N$ are tangent. 
Proof of Corollary 3.33. The "only if" part follows from the fact that $M=$ $\int x \mathrm{~d} \bar{\mu}^{M}$ and $N=\int x \mathrm{~d} \bar{\mu}^{N}$ by Theorem 3.30, the fact that $\nu^{M}=\nu^{N}$ as $M$ and $N$ are tangent, and so Theorem 3.22, Definition 3.28, and Lemma 3.34

$$
\begin{aligned}
\mathbb{E} \sup _{t \geq 0}\left\|M_{t}\right\|^{p} & =\mathbb{E} \sup _{t \geq 0}\left\|\int_{[0, t] \times X} x \mathrm{~d} \bar{\mu}^{M}\right\|^{p} \bar{\sim}_{p, X} \mathbb{E} \sup _{t \geq 0}\left\|\int_{[0, t] \times X} x \mathrm{~d} \bar{\mu}_{\text {Cox }}\right\|^{p} \\
& \bar{\sim}_{p, X} \mathbb{E} \sup _{t \geq 0}\left\|\int_{[0, t] \times X} x \mathrm{~d} \bar{\mu}^{N}\right\|^{p}=\mathbb{E} \sup _{t \geq 0}\left\|N_{t}\right\|^{p}
\end{aligned}
$$

(here we use the fact that both $\mu^{M}$ and $\mu^{N}$, as they have the same compensator, can also share the same Cox process $\mu_{\text {Cox }}$ ). The "if" part follows directly from Theorem 3.22 since we can simply set $M:=\int F \mathrm{~d} \bar{\mu}$ and $N:=\int F \mathrm{~d} \bar{\mu}_{\text {Cox }}$. Then $M$ and $N$ are tangent, so in this case (3.47) in equivalent to (3.10), and thus $X$ is UMD by Theorem 3.22 .

Corollary 3.35. Let $X$ be a UMD Banach space, $M: \mathbb{R}_{+} \times \Omega \rightarrow X$ be a purely discontinuous quasi-left continuous local martingale. Let $\mu^{M}$ be a random measure defined by (2.14) with a compensator $\nu^{M}$. Then

$$
N_{t}:=\int_{[0, t] \times X} x \mathrm{~d} \bar{\mu}_{\text {Cox }}, \quad t \geq 0,
$$

is a decoupled tangent local martingale to $M$, where $\mu_{\mathrm{Cox}}$ is a Cox process directed by $\nu^{M}, \bar{\mu}_{\text {Cox }}=\mu_{\text {Cox }}-\nu^{M}$.

Proof. By a stopping time argument presented in Theorem A.3 and by Remark 2.10 we may assume that $\mathbb{E} \sup _{t \geq 0}\left\|M_{t}\right\|<\infty$. Then the corollary follows immediately from Theorem $3.22,3.30$ (so $x$ is integrable with respect to $\bar{\mu}_{\text {Cox }}$ ), and Definition 3.3, where we have that after the probability space and filtration enlargement $M$ remains a martingale with the same local characteristics thanks to Remark 3.25 and the fact that the Borel $\sigma$-algebra $\mathcal{B}(X)$ is countably generated (recall that thanks to the Pettis measurability theorem [46, Theorem 1.1.6] we may assume that $X$ is separable).

Remark 3.36. Note that $N$ constructed above has independent increments given $\nu^{M}$. Indeed, for almost any fixed $\nu^{M}$ we have that the corresponding Cox process $\mu_{\mathrm{Cox}}$ has a deterministic compensator, so it is a deterministically time changed standard Poisson random measure (see e.g. Example 3.21), and so for almost any fixed $\nu^{M}$ we have that $N\left(\nu^{M}\right)=\int x \mathrm{~d} \mu_{\text {Cox }}$ has independent increments. Therefore the desired follows from Corollary 2.7.

\subsection{Purely discontinuous martingales with accessible jumps}

The present subsection is devoted to $L^{p}$ estimates for purely discontinuous martingales with accessible jumps and to how a decoupled tangent martingale in this case look like. First we will start with the following elementary proposition which will provide us with $L^{p}$-bounds for tangent martingales. 
Proposition 3.37. Let $X$ be a Banach space, $1 \leq p<\infty$. Then $X$ is UMD if and only if for any pair $M$ and $N$ of $X$-valued tangent purely discontinuous local martingales with accessible jumps one has that

$$
\mathbb{E} \sup _{t \geq 0}\left\|M_{t}\right\|^{p} \bar{\sim}_{p, X} \mathbb{E} \sup _{t \geq 0}\left\|N_{t}\right\|^{p} .
$$

For the proof we will need the following technical lemma.

Lemma 3.38. Let $X$ be a Banach space, $M, N: \mathbb{R}_{+} \times \Omega \rightarrow X$ be tangent local martingales. Let $\tau$ be a predictable stopping times with $\tau<\infty$ a.s. Then $\mathbb{P}\left(\Delta M_{\tau} \mid \mathcal{F}_{\tau}\right)=\mathbb{P}\left(\Delta N_{\tau} \mid \mathcal{F}_{\tau}\right)$.

Proof. As $M$ and $N$ are tangent, $\nu^{M}=\nu^{N}$. In particular, since $\tau$ is predictable (and hence a process $t \mapsto \mathbf{1}_{\tau}(t)$ is predictable as well) we have that for any Borel set $A \in X$ a.s.

$$
\begin{aligned}
\mathbb{E}\left(\mathbf{1}_{A}\left(\Delta M_{\tau}\right) \mid \mathcal{F}_{\tau-}\right) \stackrel{(*)}{=} \int_{\mathbb{R}_{+}} \mathbf{1}_{\tau}(t) \mathbf{1}_{A}(x) \mathrm{d} \nu^{M}(t, x) \\
\quad=\int_{\mathbb{R}_{+}} \mathbf{1}_{\tau}(t) \mathbf{1}_{A}(x) \mathrm{d} \nu^{N}(t, x) \stackrel{(* *)}{=} \mathbb{E}\left(\mathbf{1}_{A}\left(\Delta N_{\tau}\right) \mid \mathcal{F}_{\tau-}\right),
\end{aligned}
$$

where $(*)$ follows from the fact that a.s. $\mathbf{1}_{A}\left(\Delta M_{\tau}\right)=\int_{\mathbb{R}_{+}} \mathbf{1}_{\tau}(t) \mathbf{1}_{A}(x) \mathrm{d} \mu^{M}(t, x)$, [32, Subsection 5.3], the definition of a compensator [52, Theorem I.3.17], the fact that thus both

$$
t \mapsto \mathbf{1}_{t \geq \tau} \mathbb{E}\left(\mathbf{1}_{A}\left(\Delta M_{\tau}\right) \mid \mathcal{F}_{\tau-}\right) \text { and } t \mapsto \int_{[0, t]} \mathbf{1}_{\tau}(\tau) \mathbf{1}_{A}(x) \mathrm{d} \nu^{M}(t, x), \quad t \geq 0,
$$

are compensators of $\mathbf{1}_{A}\left(\Delta M_{t}\right)$ and the uniqueness of a compensator [52, Theorem I.3.17]; $(* *)$ holds for the same reason.

Proof of Proposition 3.3\%. First notice that the "if" part follows from Lemma 3.38 , Theorem 1.1, and the fact that any discrete martingale can be represented as a continuous-time martingale having jumps at natural points (see e.g. Remark 3.2).

Let us show the "only if" part. Let $X$ be a UMD Banach space, $M$ and $N$ be $X$-valued purely discontinuous tangent martingales with accessible jumps. Then by Lemma 2.20 there exist sequences $\left(\tau_{n}^{M}\right)_{n \geq 1}$ and $\left(\tau_{n}^{N}\right)_{n \geq 1}$ of predictable stopping times with disjoint graphs such that a.s.

$$
\begin{gathered}
\{t \geq 0: \Delta M \neq 0\} \subset\left\{\tau_{1}^{M}, \ldots, \tau_{n}^{M}, \ldots\right\}, \\
\{t \geq 0: \Delta N \neq 0\} \subset\left\{\tau_{1}^{N}, \ldots, \tau_{n}^{N}, \ldots\right\} .
\end{gathered}
$$

Moreover, by a standard merging procedure concerning predictable stopping times (see e.g. $[52,56]$ and $[32$, Subsection 5.1]) we may assume that there exists a sequence $\left(\tau_{n}\right)_{n \geq 1}$ of predictable stopping times with disjoint graphs such that a.s.

$$
\left\{\tau_{1}^{M}, \ldots, \tau_{n}^{M}, \ldots\right\} \cup\left\{\tau_{1}^{N}, \ldots, \tau_{n}^{N}, \ldots\right\} \subset\left\{\tau_{1}, \ldots, \tau_{n}, \ldots\right\} .
$$


For any $m \geq 1$ define martingales $M^{m}$ and $N^{m}$ by (B.1). Fix $\varepsilon>0$. By Proposition B.1 we can fix $m \geq 1$ to be such that

$$
\mathbb{E} \sup _{t \geq 0}\left\|M_{t}-M_{t}^{m}\right\|^{p}<\varepsilon, \quad \mathbb{E} \sup _{t \geq 0}\left\|N_{t}-N_{t}^{m}\right\|^{p}<\varepsilon .
$$

Let $\tau_{1}^{\prime}, \ldots, \tau_{m}^{\prime}$ be an increasing rearrangement of $\tau_{1}, \ldots, \tau_{m}$ (see [32, Subsection 5.3]). Then sequences $\left(d_{n}\right)_{n=1}^{2 m}$ and $\left(e_{n}\right)_{n=1}^{2 m}$

$$
\begin{aligned}
& d_{n}= \begin{cases}\Delta M_{\tau_{n / 2}^{\prime}}, & n \text { is even, } 1 \leq n \leq 2 m, \\
0, & n \text { is odd, } 1 \leq n \leq 2 m,\end{cases} \\
& e_{n}= \begin{cases}\Delta N_{\tau_{n / 2}^{\prime}}, & n \text { is even, } 1 \leq n \leq 2 m, \\
0, & n \text { is odd, } 1 \leq n \leq 2 m,\end{cases}
\end{aligned}
$$

are tangent martingale difference sequences with respect to the filtration

$$
\left(\mathcal{F}_{\tau_{1}^{\prime}-}, \mathcal{F}_{\tau_{1}^{\prime}}, \mathcal{F}_{\tau_{2}^{\prime}-}, \ldots, \mathcal{F}_{\tau_{m}^{\prime}-}, \mathcal{F}_{\tau_{m}^{\prime}}\right)
$$

Indeed, first of all the latter is a filtration by [56, Lemma 25.2]. Next notice that for any even $n=2, \ldots, 2 m$

$$
\mathbb{E}\left(d_{n} \mid \mathcal{F}_{\tau_{n / 2}^{\prime}-}\right)=\mathbb{E}\left(e_{n} \mid \mathcal{F}_{\tau_{n / 2}^{\prime}}\right)=0
$$

by Lemma 2.11. Finally for any even $n=2, \ldots, 2 m$ we have that

$$
\mathbb{P}\left(d_{n} \mid \mathcal{F}_{\tau_{n / 2}^{\prime}-}\right)=\mathbb{P}\left(\Delta M_{\tau_{n / 2}^{\prime}} \mid \mathcal{F}_{\tau_{n / 2}^{\prime}-}\right) \stackrel{(*)}{=} \mathbb{P}\left(\Delta N_{\tau_{n / 2}^{\prime}} \mid \mathcal{F}_{\tau_{n / 2}^{\prime}}\right)=\mathbb{P}\left(e_{n} \mid \mathcal{F}_{\tau_{n / 2}^{\prime}}\right),
$$

where $(*)$ follows from Lemma 3.38 and the fact that $M$ and $N$ are tangent. Therefore by the definition of $M^{m}$ and $N^{m}$, by Theorem 1.1, and by the fact that $\sup _{t \geq 0}\left\|M_{t}^{m}\right\|=\sup _{n=1}^{2 m}\left\|\sum_{k=1}^{n} d_{k}\right\|$ and $\sup _{t \geq 0}\left\|N_{t}^{m}\right\|=\sup _{n=1}^{2 m}\left\|\sum_{k=1}^{n} e_{k}\right\|$ (as both martingales $M^{m}$ and $N^{m}$ are pure jump processes which jumps coincide with $\left(d_{n}\right)_{n=1}^{2 m}$ and $\left(e_{n}\right)_{n=1}^{2 m}$ respectively) we have that

$$
\mathbb{E} \sup _{0 \leq t<\infty}\left\|M_{t}^{m}\right\|^{p} \bar{\sim}_{p, X} \mathbb{E} \sup _{0 \leq t<\infty}\left\|N_{t}^{m}\right\|^{p},
$$

and the desired follows by approaching $\varepsilon$ to zero and by Proposition B.1.

Let us now show that for any purely discontinuous martingale with accessible jumps taking values in UMD Banach spaces there exists a decoupled tangent martingale.

Theorem 3.39. Let $X$ be a UMD Banach space, $M: \mathbb{R}_{+} \times \Omega \rightarrow X$ be a purely discontinuous local martingale with accessible jumps. Then there exist an enlarged probability space $(\bar{\Omega}, \overline{\mathcal{F}}, \overline{\mathbb{P}})$ endowed with an enlarged filtration $\overline{\mathbb{F}}=$ $\left(\overline{\mathcal{F}}_{t}\right)_{t \geq 0}$, and an $\overline{\mathbb{F}}$-adapted purely discontinuous local martingale $N: \mathbb{R}_{+} \times \bar{\Omega} \rightarrow$ $X$ with accessible jumps such that $M$ is a local $\overline{\mathbb{F}}$-martingale with the same local characteristics, $M$ and $N$ are tangent and $N(\omega)$ is a martingale with independent increments and with the local characteristics $\left(0, \nu^{M}(\omega)\right)$ for a.e. $\omega \in \Omega$. 
Proof. By Remark 2.10 and Theorem A.3 The proof will be based on the construction of a $\mathrm{CI}^{5}$ tangent martingale difference sequence presented in the proof of [29, Proposition 6.1.5]. Let $\left(\tau_{n}\right)_{n \geq 1}$ be a sequence of predictable stopping times with disjoint graphs such that a.s.

$$
\left\{t \geq 0: \Delta M_{t} \neq 0\right\} \subset\left\{\tau_{1}, \ldots, \tau_{n}, \ldots\right\}
$$

(see Lemma 2.20). Let us define

$$
\bar{\Omega}:=X^{\mathbb{N}} \times \Omega, \quad \overline{\mathcal{F}}:=\left(\otimes_{n \geq 1} \mathcal{B}(X)\right) \otimes \mathcal{F},
$$

where $\mathcal{B}(X)$ is the Borel $\sigma$-algebra of $X$, and let for any $t \geq 0$ a $\sigma$-algebra $\overline{\mathcal{F}}_{t}$ on $\bar{\Omega}$ be generated by all the sets

$$
\left(B_{n} \mathbf{1}_{\tau_{n} \leq t} \cup X \mathbf{1}_{\tau_{n}>t}\right)_{n \geq 1} \times R, \quad B_{1}, \ldots, B_{n}, \ldots \in \mathcal{B}(X), \quad R \in \mathcal{F}_{t},
$$

so $\overline{\mathcal{F}}_{t}:=S_{t} \otimes \mathcal{F}_{t}$ for any $t \geq 0$ where

$$
S_{t}:=\otimes_{n \geq 1}\left(\mathcal{B}(X) \mathbf{1}_{\tau_{n} \leq t} \cup\{\varnothing, \Omega\} \mathbf{1}_{\tau_{n}>t}\right) .
$$

$\left(\overline{\mathcal{F}}_{t}\right.$ sees $x_{n}$ if $\tau_{n} \leq t$, and does not see otherwise. Note that $\otimes$ in $S_{t} \otimes \mathcal{F}_{t}$ does not mean the direct product of $\sigma$-algebras since $S_{t}$ by its definition (3.50) depends on $\Omega$, but in this case $\otimes$ means that the corresponding $\sigma$-algebra is generated by products of sets of the form (3.49)). Let $\overline{\mathbb{F}}:=\left(\overline{\mathcal{F}}_{t}\right)_{t>0}$. As $(X, \mathcal{B}(X))$ is a Polish space (see [33, pp. 344, 386]), by [33, Theorem 10.2.2] for any $n \geq 1$ and for almost any $\omega \in \Omega$ there exists a probability measure $\mathbb{P}_{\omega}^{n}$ on $X$ such that for any $B \in \mathcal{B}(X)$ (see $(2.5)$ for the definition of $\mathcal{F}_{\tau-}$ )

$$
\mathbb{E}\left(\mathbf{1}_{B}\left(\Delta M_{\tau_{n}}\right) \mid \mathcal{F}_{\tau_{n}-}\right)(\omega)=\mathbb{P}_{\omega}^{n}(B), \quad \omega \in \Omega .
$$

Then set

$$
\overline{\mathbb{P}}(A \times R):=\int_{R} \otimes_{n \geq 1} \mathbb{P}_{\omega}^{n}(A) d \mathbb{P}(\omega), \quad A \in X^{\mathbb{N}}, \quad R \in \mathcal{F} .
$$

Now let us construct a càdlàg process $N: \mathbb{R}_{+} \times \bar{\Omega} \rightarrow X$ satisfying for a.e. $\omega \in \bar{\Omega}$

$$
\Delta N_{\tau_{n}}\left(\left(x_{i}\right)_{i \geq 1}, \omega\right)=x_{n}, \quad\left(x_{i}\right)_{i \geq 1} \in X^{\mathbb{N}} .
$$

(Spoiler: this is going to be our decoupled tangent martingale). We need to show that such a process exists $\overline{\mathbb{P}}$-a.s. and that this is an $\overline{\mathbb{F}}$-martingale. For each $m \geq 1$ define $N^{m}: \mathbb{R}_{+} \times \bar{\Omega} \rightarrow X$ to be

$$
N_{t}^{m}\left(\left(x_{n}\right)_{n \geq 1}, \omega\right):=\sum_{n=1}^{m} x_{n} \mathbf{1}_{[0, t]}\left(\tau_{n}\right), \quad t \geq 0, \quad\left(x_{n}\right)_{n \geq 1} \in X^{\mathbb{N}}, \omega \in \Omega .
$$

First note that $N^{m}$ is an $\overline{\mathbb{F}}$-adapted process with values in $X$ as for any fixed $t \geq 0$

$$
\left(t,\left(x_{n}\right)_{n \geq 1}, \omega\right) \mapsto x_{n} \mathbf{1}_{[0, t]}\left(\tau_{n}\right), \quad\left(x_{n}\right)_{n \geq 1} \in X^{\mathbb{N}}, \omega \in \Omega,
$$

\footnotetext{
${ }^{5} \mathrm{CI}$ is for conditionally independent
} 
is $\overline{\mathcal{F}}_{t}$-measurable since $\overline{\mathcal{F}}_{t}=S_{t} \otimes \mathcal{F}_{t}$ with $S_{t}$ defined by (3.50), so $N^{m}$ is $\overline{\mathbb{F}}$ adapted as a sum of $\overline{\mathbb{F}}$-adapted processes.

Let us show that $N^{m}$ is a purely discontinuous martingale with accessible jumps. $N^{m}$ has accessible jumps as by the definition (3.54) of $N^{m}$ it jumps only at predictable stopping times $\left\{\tau_{1}, \ldots, \tau_{m}\right\}$ (which remain predictable stopping times with respect to the enlarged filtration $\overline{\mathbb{F}}$ as they remain being announced by the same sequences of stopping times, see Subsection 2.4). Note that for any $1 \leq n \leq m$ we have that (here $\overline{\mathcal{F}}_{\tau_{n}-}$ and $S_{\tau_{n}-}$ are defined analogously $\mathcal{F}_{\tau_{n}-}$ through an announcing sequence as $\tau_{n}$ is a predictable stopping time, see Subsection 2.4 and (3.55))

$\mathbb{E}\left(\Delta N_{\tau_{n}}^{m} \mid \overline{\mathcal{F}}_{\tau_{n}-}\right)=\mathbb{E}\left(\Delta N_{\tau_{n}}^{m} \mid S_{\tau_{n}-} \otimes \mathcal{F}_{\tau_{n}-}\right)=\mathbb{E}\left(\mathbb{E}\left(\Delta N_{\tau_{n}}^{m} \mid S_{\tau_{n}-} \otimes \mathcal{F}\right) \mid S_{\tau_{n}-} \otimes \mathcal{F}_{\tau_{n}-}\right)$.

(Here $\otimes$ again $i s$ not a product of $\sigma$-algebras, but a $\sigma$-algebra generated by products of sets of the form familiar to (3.49)). We need to show that $\mathbb{E}\left(\Delta N_{\tau_{n}}^{m} \mid S_{\tau_{n}-} \otimes\right.$ $\mathcal{F})=0$. It is sufficient to show that for $\overline{\mathbb{P}}$-almost any fixed $\omega \in \bar{\Omega}$, $\mathbb{E}\left(\Delta N_{\tau_{n}(\omega)}^{m}(\omega) \mid S_{\tau_{n}-}\right)=0$ because we have that for any $R \in \mathcal{F}$ and $A \times R \in$ $S_{\tau_{n}-} \otimes \mathcal{F}$ (where $A$ depends on $\Omega$ in a predictable way so that $A \times R$ has the form (3.49)) we have that

$$
\int_{A \times R} \Delta N_{\tau_{n}}^{m} \mathrm{~d} \overline{\mathbb{P}}=\int_{R} \int_{A} \Delta N_{\tau_{n}}^{m} \mathrm{~d} \otimes_{n \geq 1} \mathbb{P}_{n}^{\omega} \mathrm{d} \mathbb{P}(\omega),
$$

so the first integral equals zero if $\int_{A} \Delta N_{\tau_{n}}^{m} \mathrm{~d} \otimes_{n \geq 1} \mathbb{P}_{n}^{\omega}=0$ for a.e. $\omega \in \Omega$ for any $A \in S_{\tau_{n}-}$. By the definition (3.50) of $S_{t}$ we have that for almost any fixed $\omega \in \Omega$

$$
\begin{aligned}
S_{\tau_{n}} & =\otimes_{i \geq 1, \tau_{i} \leq \tau_{n}} \mathcal{B}(X) \otimes_{i \geq 1, \tau_{i}>\tau_{n}}\{\varnothing, \Omega\}, \\
S_{\tau_{n}-} & =\otimes_{i \geq 1, \tau_{i}<\tau_{n}} \mathcal{B}(X) \otimes_{i \geq 1, \tau_{i} \geq \tau_{n}}\{\varnothing, \Omega\},
\end{aligned}
$$

so we have that

$$
S_{\tau_{n}}=\sigma\left(S_{\tau_{n}-}, \Delta S_{\tau_{n}}\right), \quad \Delta S_{\tau_{n}}:=\otimes_{i \geq 1}\left(\mathcal{B}(X) \mathbf{1}_{i=n}\right) \cup\left(\{\varnothing, \Omega\} \mathbf{1}_{i \neq n}\right),
$$

(here we used the fact that $\left(\tau_{n}\right)_{n \geq 1}$ have a.s. disjoint graphs), so $S_{\tau_{n}}$ is a.s. generated by two independent $\sigma$-algebras $S_{\tau_{n}-}$ and $\Delta S_{\tau_{n}}$ (which are independent a.s. by the definition (3.52) of $\overline{\mathbb{P}}$ ), and hence as $\Delta N_{\tau_{n}}^{m}$ is a.s. $\Delta S_{\tau_{n}}$-measurable, $\mathbb{E}\left(\Delta N_{\tau_{n}}^{m}(\omega) \mid S_{\tau_{n}-}\right)=\mathbb{E}_{X^{\mathbb{N}}}\left(\Delta N_{\tau_{n}}^{m}\right)(\omega)$. Finally note that $\Delta N_{\tau_{n}}^{m}(\omega)$ has $\mathbb{P}_{\omega}^{n}$ as its distribution by the definition (3.52) of $\overline{\mathbb{P}}$ and the definition (3.54) of $N^{m}$, and the latter distribution has a.s. a mean zero by the definition (3.51) as $\int_{X} x \mathrm{~d} \mathbb{P}_{\omega}^{n}=$ $\mathbb{E}\left(\Delta M_{\tau_{n}} \mid \mathcal{F}_{\tau_{n}-}\right)(\omega)=0$ for a.e. $\omega \in \Omega$. Therefore $\mathbb{E}\left(\Delta N_{\tau_{n}}^{m} \mid \overline{\mathcal{F}}_{\tau_{n}-}\right)=0$, and hence $N^{m}$ is a martingale by [32, Subsection 5.3].

Let us now show that $M$ is an $\overline{\mathbb{F}}$-martingale with the same local characteristics $\left(0, \nu^{M}\right)$. Fix $n \geq 1$. Then for any Borel $B \subset X$ and any $\overline{\mathcal{F}}_{\tau_{n}-\text {-measurable }}$ bounded $F: \bar{\Omega} \rightarrow \mathbb{R}$ we have that

$$
\mathbb{E} \mathbf{1}_{B}\left(\Delta M_{\tau_{n}}\right) F=\mathbb{E} \mathbb{E}_{\omega} \mathbf{1}_{B}\left(\Delta M_{\tau_{n}}\right)(\omega) F=\mathbb{E} \mathbf{1}_{B}\left(\Delta M_{\tau_{n}}\right) \mathbb{E}_{\omega} F,
$$


where $\mathbb{E}_{\omega}$ denotes expectation w.r.t. $\otimes_{n \geq 1} \mathbb{P}_{\omega}^{n}$ for each fixed $\omega \in \Omega$. As $F$ is $\overline{\mathcal{F}}_{\tau_{n}-}$-measurable and as $\overline{\mathcal{F}}_{\tau_{n}-} \subset S_{\infty} \otimes \mathcal{F}_{\tau_{n}-}$ (this follows due to that fact that $\left.\overline{\mathcal{F}}_{\tau_{n}-}=S_{\tau_{n}-} \otimes \mathcal{F}_{\tau_{n}-}\right)$ we have that $\mathbb{E}_{\omega} F$ is $\mathcal{F}_{\tau_{n}-}$-measurable and hence

$$
\mathbb{E} \mathbf{1}_{B}\left(\Delta M_{\tau_{n}}\right) \mathbb{E}_{\omega} F=\mathbb{E} \mathbb{E}\left(\mathbf{1}_{B}\left(\Delta M_{\tau_{n}}\right) \mid \mathcal{F}_{\tau_{n}-}\right) \mathbb{E}_{\omega} F=\mathbb{E} \mathbb{E}\left(\mathbf{1}_{B}\left(\Delta M_{\tau_{n}}\right) \mid \mathcal{F}_{\tau_{n}-}\right) F,
$$

so by the definition of conditional expectation and freedom in choice of $F$ we have that

$$
\begin{aligned}
\overline{\mathbb{P}}\left(\left(\Delta M_{\tau_{n}}\right) \mid \overline{\mathcal{F}}_{\tau_{n}-}\right)(B) & =\mathbb{E}\left(\mathbf{1}_{B}\left(\Delta M_{\tau_{n}}\right) \mid \overline{\mathcal{F}}_{\tau_{n}-}\right) \\
& =\mathbb{E}\left(\mathbf{1}_{B}\left(\Delta M_{\tau_{n}}\right) \mid \mathcal{F}_{\tau_{n}-}\right)=\overline{\mathbb{P}}\left(\left(\Delta M_{\tau_{n}}\right) \mid \overline{\mathcal{F}}_{\tau_{n}-}\right)(B),
\end{aligned}
$$

and hence $M$ has the same $\overline{\mathbb{F}}$-local characteristics $\left(0, \nu^{M}\right)$. The fact that $M$ is an $\overline{\mathbb{F}}$-martingale follows from Lemma 2.11 , the fact that $\left(\tau_{n}\right)_{n \geq 1}$ exhausts all the jumps of $M$, and the fact that by (3.58)

$$
\mathbb{E}\left(\Delta M_{\tau_{n}} \mid \overline{\mathcal{F}}_{\tau_{n}-}\right)=\mathbb{E}\left(\Delta M_{\tau_{n}} \mid \mathcal{F}_{\tau_{n}-}\right)=0 .
$$

Now let $M^{m}$ be defined by (B.1) and let us show that $N^{m}$ is a decoupled tangent martingale to $M^{m}$. Note that $M^{m}$ is an $\overline{\mathbb{F}}$-martingale as well as $M$. First $M^{m}$ and $N^{m}$ have jumps only at $\left\{\tau_{1}, \ldots, \tau_{m}\right\}$, so they are tangent because for any $1 \leq n \leq m$ for any $B \in \mathcal{B}(X)$ for a.e. $\omega \in \bar{\Omega}$

$$
\begin{aligned}
\mathbb{P}\left(\Delta M_{\tau_{n}}^{m} \mid \overline{\mathcal{F}}_{\tau_{n}-}\right)(B)(\omega) \stackrel{(i)}{=} \mathbb{P}\left(\Delta M_{\tau_{n}} \mid \overline{\mathcal{F}}_{\tau_{n}-}\right)(B)(\omega) \\
\quad=\mathbb{E}\left(\mathbf{1}_{B}\left(\Delta M_{\tau_{n}}\right) \mid \overline{\mathcal{F}}_{\tau_{n}-}\right)(\omega) \stackrel{(i i)}{=} \mathbb{P}_{\omega}^{n}(B) \stackrel{(i i i)}{=} \mathbb{P}\left(\Delta N_{\tau_{n}}^{m} \mid \overline{\mathcal{F}}_{\tau_{n}-}\right)(B)(\omega),
\end{aligned}
$$

where $(i)$ follows from the definition of $M^{m}$, (ii) holds by the definition (3.51) of $\mathbb{P}_{\omega}^{n}$, and (iii) follows from the definition of $\overline{\mathbb{P}}$ and the definition of $N^{m}$.

Let us show that $N^{m}$ is a decoupled tangent martingale to $M^{m}$, i.e. that $N^{m}(\omega)$ has independent mean-zero increments and local characteristics $\left(0, \nu^{M^{m}}(\omega)\right)$ for a.e. $\omega \in \Omega$. This easily follows from the fact that for a.e. fixed $\omega \in \Omega$ the process $N^{m}(\omega)$ has fixed jumps at $\left\{\tau_{1}(\omega), \ldots, \tau_{m}(\omega)\right\}$ and for every $1 \leq n \leq m$ we have that $\Delta N_{\tau_{n}}^{m}(\omega)$ is $\Delta S_{\tau_{n}(\omega)}$-measurable; as $S_{\infty}=$ $\sigma\left(\Delta S_{\tau_{n}(\omega)}, n \geq 1\right)=\otimes_{n \geq 1} \mathcal{B}(X)$, so $\left(\Delta N_{\tau_{n}}^{m}(\omega)\right)_{n=1}^{m}$ are independent since $\left(\Delta S_{\tau_{n}(\omega)}\right)_{n \geq 1}$ are independent. The fact that $N^{m}(\omega)$ has local characteristics $\left(0, \nu^{M^{m}}(\omega)\right)$ follows from the construction of $N^{m}$.

Now let us show that $N^{m}$ converges as $m \rightarrow \infty$, and that the limit coincides with the desired $N$ which thus exists. For any $m_{2} \geq m_{1} \geq 1$ by (3.48) and by the fact that $N^{m_{1}}-N^{m_{2}}$ is a decoupled tangent martingale to $M^{m_{1}}-M^{m_{2}}$ (which can be shown analogously to the considerations above) we have that

$$
\mathbb{E} \sup _{t \geq 0}\left\|N_{t}^{m_{1}}-N_{t}^{m_{2}}\right\| \bar{\sim}_{X} \mathbb{E} \sup _{t \geq 0}\left\|M_{t}^{m_{1}}-M_{t}^{m_{2}}\right\| .
$$

Thus martingales $\left(N^{m}\right)_{m \geq 1}$ converge in $L^{1}\left(\bar{\Omega} ; \mathcal{D}\left(\mathbb{R}_{+}, X\right)\right)$ by (B.4). Let $\widetilde{N}$ be the limit. Note that by Theorem $2.9 \widetilde{N}$ is an $\overline{\mathbb{F}}$-martingales. Let us show that 
$\widetilde{N}$ coincides with the desired $N$. For any $n \geq 1$ we have that for $\Delta N_{\tau_{n}}$ defined by (3.53) (note that we still need to prove that $N$ exists and that $\widetilde{N}=N$ ) and by the fact that $\Delta N_{\tau_{n}}=\Delta N_{\tau_{n}}^{m}$ for $m \geq n$

$$
\begin{aligned}
\mathbb{E}\left\|\Delta \widetilde{N}_{\tau_{n}}-\Delta N_{\tau_{n}}\right\| & =\lim _{m \rightarrow \infty} \mathbb{E}\left\|\Delta \widetilde{N}_{\tau_{n}}-\Delta N_{\tau_{n}}^{m}\right\| \\
\stackrel{(*)}{\leq} \lim _{m \rightarrow \infty} \mathbb{E}\left(\left\|\widetilde{N}_{\tau_{n}}-N_{\tau_{n}}^{m}\right\|+\right. & \left.\left\|\widetilde{N}_{\tau_{n}-}-N_{\tau_{n}-}^{m}\right\|\right) \\
& \leq 2 \lim _{m \rightarrow \infty} \mathbb{E} \sup _{t \geq 0}\left\|\widetilde{N}_{t}-N_{t}^{m}\right\|=0
\end{aligned}
$$

where $(*)$ follows by a triangle inequality. For the same reason we have that $\Delta \widetilde{N}_{\tau}=0$ a.s. on $\tau \notin\left\{\tau_{1}, \ldots, \tau_{n}, \ldots\right\}$ for any stopping time $\tau$. Therefore $\widetilde{N}$ coincides with the desired $N$, so such $N$ exists. $N$ is a decoupled tangent martingale to $M$ for the same reason as $N^{m}$ is a decoupled tangent martingale to $M^{m}$ for any $m \geq 1$.

Remark 3.40. To sum up Theorem 3.39. Any purely discontinuous martingale $M$ with accessible jumps and with values in a UMD Banach space has a tangent martingale $N$ on an enlarged probability space with an enlarged filtration such that for a.e. $\omega$ from the original probability space $N$ is a martingale with fixed jump times coinciding with the jumps times of $M$ and with independent increments.

Remark 3.41. Note that $N$ constructed in the proof of Theorem 3.39 has independent increments given $\nu^{M}$. Indeed, for a.e. fixed $\nu^{M}$ we have that the set $\left(\tau_{n}(\cdot)\right)_{n \geq 1}$ is fixed, the distributions $\left(\mathbb{P}_{\omega}^{n}\right)_{n \geq 1}$ are fixed and mean zero, so $\left(\Delta N_{\tau_{n}(\cdot)}\right)_{n \geq 1}$ are independent mean-zero random variable. Consequently the desired independence follows from Corollary 2.7.

\subsection{Proof of Theorem 3.7 and 3.8}

Let us finally prove Theorem 3.7 and 3.8. First, Theorem 3.7 follows from Theorem 2.18, Remark 2.19, Proposition 3.17, 3.37, and Corollary 3.33. Now let us show Theorem 3.8. This theorem follows from the fact that for a.e. fixed $\omega \in \Omega$ we have that $N^{c}(\omega), N^{q}(\omega)$, and $N^{a}(\omega)$ are independent. Since each of them a.s. have independent increments and local characteristics $\left(\llbracket M^{c} \rrbracket(\omega), 0\right),\left(0, \nu^{M^{q}}(\omega)\right)$, and $\left(0, \nu^{M^{a}}(\omega)\right)$ respectively, $N(\omega)$ has independent increments and local characteristics $\left(\llbracket M^{c} \rrbracket(\omega), \nu^{M}(\omega)\right)$ (the letter follows from Proposition 3.14).

It remains to show that $M$ and $N$ are local $\overline{\mathbb{F}}$-martingales with $\overline{\mathbb{F}}$-local characteristics $\left(\llbracket M^{c} \rrbracket, \nu^{M}\right)$. Let us start with $M$. To this end recall that the new filtration $\overline{\mathbb{F}}$ over the enlarged probability space $(\bar{\Omega}, \overline{\mathcal{F}}, \overline{\mathbb{P}})$ is generated by $\mathbb{F}$, time-changed independent cylindrical Wiener process $W_{H}^{\prime}$ (see the proof of Theorem 3.18), the Cox process $\mu_{\text {Cox }}$ (see Remark 3.25), and the filtration $\left(S_{t}(\omega)\right)_{t \geq 0}$ defined by (3.50). Let $F: \bar{\Omega} \rightarrow \mathbb{R}$ be any bounded $\mathcal{F}$-measurable 
random variable. Then for any fixed $t \geq 0$

$$
\begin{aligned}
\mathbb{E}\left(F \mid \overline{\mathcal{F}}_{t}\right) & =\mathbb{E}\left(\mathbb{E}\left(F \mid \sigma\left(W_{H}^{\prime}, \mathcal{N}, S_{\infty}, \mathcal{F}_{t}\right)\right) \mid \overline{\mathcal{F}}_{t}\right) \\
& \stackrel{(*)}{=} \mathbb{E}\left(\mathbb{E}\left(F \mid \mathcal{F}_{t}\right) \mid \overline{\mathcal{F}}_{t}\right)=\mathbb{E}\left(F \mid \mathcal{F}_{t}\right),
\end{aligned}
$$

where $\mathcal{N}$ is a $\sigma$-algebra generated by independent sequence of standard Poisson processes (this sequence can be assumed finite thanks to Remark 3.25), and where $(*)$ follows from the fact that $F$ is independent of $W_{H}^{\prime}$ and $\mathcal{N}$ and the trick similar to the computations (3.56) and (3.57). Hence, as $F$ was general, $M$ is a local $\overline{\mathbb{F}}$-martingale.

In order to show that $M$ preserves its local characteristics we notice by Remark 3.4 that $\llbracket M^{c} \rrbracket$ stays the same, the predictable jumps $\left(\tau_{n}\right)_{n \geq 1}$ remain predictable (hence $M^{a}$ has accessible jumps and the local characteristics $\left(0, \nu^{M^{a}}\right)$ do not change by (3.56) and (3.57)), and $M^{q}$ does not change its local characteristics as analogously to Remark 3.25 with exploiting (3.59) instead of (3.39)

$\mu^{M^{q}}$ has the same compensator $\nu^{M^{q}}$, so $M^{q}$ has the same local characteristics $\left(0, \nu^{M^{q}}\right)$, and thus $M$ keeps the local characteristics $\left(\llbracket M^{c} \rrbracket, \nu^{M}\right)$ by Subsection 3.2 .

Proving that $N$ is a local $\overline{\mathbb{F}}$-martingales with local characteristics $\left(\llbracket M^{c} \rrbracket, \nu^{M}\right)$ follows analogously. We will only show this for $N^{c}$ (the cases of $N^{q}$ and $N^{a}$ can be shown similarly). Let $\widetilde{\mathcal{F}}_{t}:=\left.\mathcal{F}_{t} \otimes W_{H}^{\prime}\right|_{\left[0, A_{t}\right]}$ and $\widetilde{\mathbb{F}}:=\left(\widetilde{\mathcal{F}}_{t}\right)_{t \geq 0}$, where the time-change $\left(A_{t}\right)_{t \geq 0}$ depending only on $\mathcal{F}_{t}$ defined in the proof of Theorem 3.18. Then for any bounded $\widetilde{\mathcal{F}}_{\infty}$-measurable $F$ similarly to (3.59)

$$
\mathbb{E}\left(F \mid \overline{\mathcal{F}}_{t}\right)=\mathbb{E}\left(\mathbb{E}\left(F \mid \sigma\left(\mathcal{N}, S_{\infty}, \widetilde{\mathcal{F}}_{t}\right)\right) \mid \overline{\mathcal{F}}_{t}\right)=\mathbb{E}\left(\mathbb{E}\left(F \mid \widetilde{\mathcal{F}}_{t}\right) \mid \overline{\mathcal{F}}_{t}\right)=\mathbb{E}\left(F \mid \widetilde{\mathcal{F}}_{t}\right),
$$

so as $N^{c}$ is a local $\widetilde{\mathbb{F}}$-martingale, it is a local $\overline{\mathbb{F}}$-martingale. The fact that $N$ has the local characteristics $\left(\llbracket M^{c} \rrbracket, \nu^{M}\right)$ follows in the same way as for $M$.

\subsection{Uniqueness of a decoupled tangent martingale}

This subsection is devoted to showing that a decoupled tangent local martingale, if exists, is unique up to the distribution.

Proposition 3.42. Let $X$ be a Banach space, $M: \mathbb{R}_{+} \times \Omega \rightarrow X$ be a local martingale. Assume that $M$ has two decoupled tangent local martingales $N^{1}$ and $N^{2}$ on possibly different enlarged probability spaces with enlarged filtrations. Then $N^{1}$ and $N^{2}$ are equidistributed as random elements with values in $\mathcal{D}\left(\mathbb{R}_{+}, X\right)$.

Proof. Suppose that $N^{1}$ and $N^{2}$ live on probability spaces $\left(\bar{\Omega}^{1}, \overline{\mathcal{F}}^{1}, \overline{\mathbb{P}}^{1}\right)$ and $\left(\bar{\Omega}^{2}, \overline{\mathcal{F}}^{2}, \overline{\mathbb{P}}^{2}\right)$ respectively, where both $\left(\bar{\Omega}^{1}, \overline{\mathcal{F}}^{1}, \overline{\mathbb{P}}^{1}\right)$ and $\left(\bar{\Omega}^{2}, \overline{\mathcal{F}}^{2}, \overline{\mathbb{P}}^{2}\right)$ are enlargements of $(\Omega, \mathcal{F}, \mathbb{P})$ (see Definition 2.3). Then by Definition 3.3 for a.e. fixed $\omega \in \Omega$ processes $N^{1}(\omega)$ and $N^{2}(\omega)$ are local martingales with independent increments and local characteristics $\left(\llbracket M^{c} \rrbracket(\omega), \nu^{M}(\omega)\right)$. Thus $N^{1}(\omega)$ and $N^{2}(\omega)$ are equidistributed by Corollary 9.8, and thus $N^{1}$ and $N^{2}$ are equidistributed as we have that for any Borel set $B \in \mathcal{D}\left(\mathbb{R}_{+}, X\right)$ 


$$
\begin{aligned}
\overline{\mathbb{P}}^{1}\left(N^{1} \in B\right)=\int_{\Omega} \widehat{\mathbb{P}}_{\omega}^{1}\left(N^{1}(\omega) \in B\right) \mathrm{d} \mathbb{P}(\omega) \\
\quad=\int_{\Omega} \widehat{\mathbb{P}}_{\omega}^{2}\left(N^{2}(\omega) \in B\right) \mathrm{d} \mathbb{P}(\omega)=\overline{\mathbb{P}}^{2}\left(N^{2} \in B\right),
\end{aligned}
$$

where $\widehat{\mathbb{P}}_{\omega}^{1}$ and $\widehat{\mathbb{P}}_{\omega}^{2}$ are as in Definition 2.3. This terminates the proof.

\subsection{Independent increments given the local characteristics}

In fact, we can make Definition 3.3 stronger by proving the following theorem.

Theorem 3.43. Let $X$ be a UMD Banach space, $M: \mathbb{R}_{+} \times \Omega \rightarrow X$ be a local martingale, $N: \mathbb{R}_{+} \times \bar{\Omega} \rightarrow X$ be a decoupled tangent local martingale to $M$. Then $N$ has independent increments given $\left(\llbracket M^{c} \rrbracket, \nu^{M}\right)$.

This theorem extends e.g. [29, Example 6.1.7] and [58, Theorem 3.1].

Proof. The theorem follows directly from the construction of a decoupled tangent local martingale presented in Theorem 3.18, 3.22, and 3.39, from Remark 3.20,3.36, and 3.41, from that fact that we can consider an enlargement of $(\Omega, \mathcal{F}, \mathbb{P})$ generated by $W_{H}^{\prime}, \mu_{\mathrm{Cox}}$, and $\overline{\mathbb{P}}$ defined by $(3.52)$, and from Corollary 2.7 on condtioinal independence with respect to a random variable.

\section{Upper bounds and the decoupling property}

As it was shown in Theorem 3.7, if $X$ is UMD, then for any local martingale $M$ and for a decoupled tangent local martingale $N$ we have that for any $1 \leq p<\infty$

$$
\mathbb{E} \sup _{0 \leq t \leq T}\left\|M_{t}\right\|^{p} \bar{\sim}_{p, X} \mathbb{E} \sup _{0 \leq t \leq T}\left\|N_{t}\right\|^{p} \stackrel{(*)}{\sim}_{p} \mathbb{E}\left\|N_{T}\right\|^{p}, \quad T>0
$$

where $(*)$ follows from Lemma 9.3 and the fact that $N(\omega)$ is a martingale with independent increments for a.e. $\omega \in \Omega$. But what if we are interested only in the upper bound of (4.1) (this is often the case, see Remark 6.5 on stochastic integration)? Can we have such estimates for non-UMD Banach spaces? Inequalities of such form have been discovered by Cox and Veraar in $[25,26]$ (see also $[24,46,73])$ and they turn out to characterize the so-called decoupling property.

Definition 4.1. Let $X$ be a Banach space. Then $X$ is said to have the decoupling property if for any $1 \leq p<\infty$, for any $X$-valued martingale difference sequence $\left(d_{n}\right)_{n \geq 1}$ and for a decoupled tangent martingale difference sequence $\left(e_{n}\right)_{n \geq 1}$ one has that

$$
\mathbb{E} \sup _{N \geq 1}\left\|\sum_{n=1}^{N} d_{n}\right\|^{p} \lesssim_{p, X} \mathbb{E}\left\|\sum_{n=1}^{\infty} c_{n}\right\|^{p}
$$


Unlike the UMD property, Banach spaces with the decoupling property might not enjoy reflexivity. For example, $L^{1}$ spaces has the decoupling property. Moreover, quasi-Banach spaces can also satisfy $(4.2)$ (e.g. $L^{q}$ for $q \in(0,1)$, see [26]).

The goal of the present section is to extend (4.2) to the continuous-times case. Of course for a general Banach space $X$ with a decoupling property and for a general $X$-valued martingale we will not have a decoupled tangent local martingale thanks to Theorem 3.8, but nonetheless, we are able to provide a continuoustime analogue of (4.2) in some spacial cases when such a decouple tangent local martingale exists. Let us start with the continuous case which is an elementary consequence of [26, Theorem 5.4]. Recall that for any time change $\left(\tau_{s}\right)_{s \geq 0}$ we have the inverse time change $\left(A_{t}\right)_{t \geq 0}$ defined by $A_{t}:=\inf \left\{s \geq 0: \tau_{s} \geq t\right\}$, and that a process is in $\gamma_{\text {loc }}\left(L^{2}\left(\mathbb{R}_{+} ; H\right), X\right)$ if it is locally in $\gamma\left(L^{2}\left(\mathbb{R}_{+} ; H\right), X\right)$ (see Subsection 2.11).

Theorem 4.2. Let $X$ be a Banach space with the decoupling property, $M^{c}$ : $\mathbb{R}_{+} \times \Omega \rightarrow X$ be a continuous local martingale. Assume that there exists a time change $\left(\tau_{s}^{c}\right)_{s \geq 0}$, a Hilbert space $H$, an $H$-cylindrical Brownian motion $W_{H}$ adapted with respect to (possibly, enlarged) filtration $\mathbb{G}:=\left(\mathcal{F}_{\tau_{s}}\right)_{s \geq 0}$, and a strongly $\mathbb{G}$-predictable process $\Phi: \Omega \rightarrow \gamma_{\text {loc }}\left(L^{2}\left(\mathbb{R}_{+} ; H\right), X\right)$ such that for any $x^{*} \in X^{*}$ we have that $\left\langle M^{c}, x^{*}\right\rangle \circ \tau^{c}=\Phi^{*} x^{*} \cdot W_{H}$ a.s. Then $M^{c}$ has a decoupled tangent local martingale $N^{c}$ which has the following form: $N^{c}=\left(\Phi \cdot \bar{W}_{H}\right) \circ A^{c}$, where $\bar{W}_{H}$ is an independent copy of $W_{H}$ and $\left(A_{t}^{c}\right)_{t \geq 0}$ is the time change inverse to $\tau^{c}$. Moreover, if this is the case then for any $1 \leq p<\infty$

$$
\mathbb{E} \sup _{0 \leq t \leq T}\left\|M_{t}^{c}\right\|^{p} \lesssim_{p, X} \mathbb{E}\left\|N_{T}^{c}\right\|^{p}, \quad T \geq 0 .
$$

Proof. First note that by [26, Theorem 5.4] $\Phi$ is integrable with respect to $W_{H}$ and $M^{c} \circ \tau^{c}=\Phi \cdot W_{H}$. Moreover, by [26, Theorem 5.4] we also have that $\Phi$ is integrable with respect to $\bar{W}_{H}$. Let $N^{c}:=\left(\Phi \cdot \bar{W}_{H}\right) \circ A^{c}$. Then $N^{c}$ is a decoupled tangent local martingale to $M^{c}$ due to Definition 3.3 and (2.17). (4.3) follows directly from $[26,(5.3)]$ and the fact that

$$
\mathbb{E}_{\bar{W}_{H}}\left\|N_{T}^{c}\right\|^{p}=\mathbb{E}_{\bar{W}_{H}}\left\|\int_{0}^{A_{t}} \Phi \mathrm{d} \bar{W}_{H}\right\|^{p} \bar{\sim}_{p} \mathbb{E}\|\Phi\|_{\gamma\left(L^{2}\left(\left[0, A_{t}\right] ; H\right), X\right)}^{p} .
$$

Now let us move to the quasi-left continuous case. Recall that a stochastic integral with respect to a random measure was defined in Definition 3.28.

Theorem 4.3. Let $X$ be a Banach space satisfying the decoupling property, $(J, \mathcal{J})$ be a measurable space. Let $\mu$ be a $\widetilde{\mathcal{P}}-\sigma$-finite quasi-left continuous integer random measure on $\mathbb{R}_{+} \times J$ with a compensator $\nu, \bar{\mu}:=\mu-\nu$. Let $F: \mathbb{R}_{+} \times \Omega \rightarrow$ $X$ be strongly $\widetilde{\mathcal{P}}$-measurable. Assume that $F(\omega)$ is integrable with respect to $\bar{\mu}_{\mathrm{Cox}}(\omega)$ for a.e. $\omega \in \Omega$, where $\mu_{\mathrm{Cox}}$ is a Cox process directed by $\nu, \bar{\mu}_{\mathrm{Cox}}=$ $\mu_{\mathrm{Cox}}-\nu$. Then $F$ is locally integrable with respect to $\bar{\mu}$ and for any $1 \leq p<\infty$

$$
\mathbb{E} \sup _{t \geq 0}\left\|\int_{[0, t] \times J} F \mathrm{~d} \bar{\mu}\right\|^{p} \lesssim_{p, X} \mathbb{E}\left\|\int_{R_{+} \times J} F \mathrm{~d} \bar{\mu}_{\text {Cox }}\right\|^{p} .
$$


Proof. For each $k \geq 1$ define a stopping time

$$
\tau_{k}:=\inf \left\{t \geq 0: \mathbb{E}_{\text {Cox }}\left\|\int_{[0, t] \times J} F \mathrm{~d} \bar{\mu}_{\text {Cox }}\right\|=k\right\} .
$$

We can find such (possibly infinite) $t$ that $\mathbb{E}_{\text {Cox }}\left\|\int_{[0, t] \times J} F \mathrm{~d} \bar{\mu}_{\text {Cox }}\right\|=k$ since the function $t \mapsto \mathbb{E}_{\text {Cox }}\left\|\int_{[0, t] \times J} F(\omega) \mathrm{d} \bar{\mu}_{\mathrm{Cox}}(\omega)\right\|$ is continuous in $t \geq 0$ for a.e. $\omega \in \Omega$ because $\int_{[t, t+\varepsilon] \times J} F(\omega) \mathrm{d} \bar{\mu}_{\mathrm{Cox}}(\omega) \rightarrow 0$ a.s. as $\varepsilon \rightarrow 0$. Without loss of generality by a stopping time argument we can set $F:=F \mathbf{1}_{\left[0, \tau_{k}\right]}$ and so we may assume that $\mathbb{E}_{\text {Cox }}\left\|\int_{\mathbb{R}_{+} \times J} F \mathrm{~d} \bar{\mu}_{\text {Cox }}\right\|<C$ a.s. for some $C>0$.

Let us show that $F$ is integrable with respect to $\bar{\mu}$. Let the sets $\left(A_{n}\right)_{n \geq 1}$ be as in Definition 3.28. Then

$$
t \mapsto M_{t}^{n}:=\int_{[0, t] \times J} F \mathbf{1}_{A_{n}} \mathrm{~d} \bar{\mu}, \quad t \geq 0,
$$

is a martingale for any $n \geq 1$. Due to (4.2) and by an approximating by step functions we have that

$$
\mathbb{E} \sup _{t \geq 0}\left\|M_{t}^{n}\right\|^{p} \lesssim p, X \mathbb{E} \mathbb{E}_{\operatorname{Cox}}\left\|\int_{\mathbb{R}_{+} \times \Omega} F \mathbf{1}_{A_{n}} \mathrm{~d} \bar{\mu}_{\text {Cox }}\right\|^{p}, \quad n \geq 1,
$$

and for the same reason for any $m \geq n \geq 1$

$$
\mathbb{E} \sup _{t \geq 0}\left\|M_{t}^{m}-M_{t}^{n}\right\| \lesssim X \mathbb{E} \mathbb{E}_{\operatorname{Cox}}\left\|\int_{\mathbb{R}_{+} \times \Omega} F \mathbf{1}_{A_{m} \backslash A_{n}} \mathrm{~d} \bar{\mu}_{\text {Cox }}\right\| .
$$

Thus we have that $\left(M^{n}\right)_{n \geq 1}$ is a Cauchy sequence in $L^{1}\left(\Omega ; \mathcal{D}\left(\mathbb{R}_{+}, X\right)\right)$ by (4.6) and Remark 2.26. Inequality (4.4) follows from (4.5) by letting $n \rightarrow \infty$.

The following corollary is a direct consequence of Theorem 4.3.

Corollary 4.4. Let $X$ be a Banach space with the decoupling property, $M^{q}$ : $\mathbb{R}_{+} \times \Omega \rightarrow X$ be a purely discontinuous quasi-left continuous local martingale. Let $\mu^{M^{q}}$ be defined by (2.14), $\nu^{M^{q}}$ be the corresponding compensator, $\mu_{\mathrm{Cox}}^{M^{q}}$ be a Cox process directed by $\nu^{M^{q}}, \bar{\mu}_{\mathrm{Cox}}^{M^{q}}:=\mu_{\mathrm{Cox}}^{M^{q}}-\nu^{M^{q}}$. Assume that $\int_{[0, t] \times X} x \mathrm{~d} \bar{\mu}_{\mathrm{Cox}}^{M^{q}}$ is well defined a.s. for any $t \geq 0$. Then an $X$-valued local martingale $N^{q}$ defined by

$$
N_{t}^{q}:=\int_{[0, t] \times X} x \mathrm{~d} \bar{\mu}_{\text {Cox }}^{M^{q}}, \quad t \geq 0,
$$

is a decoupled tangent local martingale to $M^{q}$ and for any $1 \leq p<\infty$

$$
\mathbb{E} \sup _{0 \leq t \leq T}\left\|M_{t}^{q}\right\|^{p} \lesssim_{p, X} \mathbb{E}\left\|N_{T}^{q}\right\|^{p}, \quad T \geq 0 .
$$

Now let us move to the accessible jump case. 
Theorem 4.5. Let $X$ be a Banach space with the decoupling property, $M^{a}$ : $\mathbb{R}_{+} \times \Omega \rightarrow X$ be a purely discontinuous local martingale with accessible jumps. Assume that it has a decoupled tangent local martingale $N^{a}$. Then for any $1 \leq$ $p<\infty$

$$
\mathbb{E} \sup _{0 \leq t \leq T}\left\|M_{t}^{a}\right\|^{p} \lesssim p, X \mathbb{E}\left\|N_{T}^{a}\right\|^{p}, \quad T \geq 0 .
$$

Proof. Fix $T>0$. Without loss of generality assume that $\mathbb{E}\left\|N_{T}^{a}\right\|^{p}<\infty$. Let $\left(\tau_{n}\right)_{n \geq 1}$ be finite predictable stopping times with disjoint graphs which exhaust jumps of $M^{a}, M^{a, m}$ and $N^{a, m}$ be defined analogously to (B.1). First notice that thanks to the proof of Proposition 3.37 and (4.2) we have that for any $m \geq 1$

$$
\mathbb{E} \sup _{0 \leq t \leq T}\left\|M_{t}^{a, m}\right\|^{p} \lesssim_{p, X} \mathbb{E}\left\|N_{T}^{a, m}\right\|^{p} .
$$

For the same reason we have that for any $\ell>m \geq 1$

$$
\mathbb{E} \sup _{0 \leq t \leq T}\left\|M_{t}^{a, \ell}-M_{t}^{a, \ell}\right\|^{p} \lesssim_{p, X} \mathbb{E}\left\|N_{T}^{a, \ell}-N_{T}^{a, m}\right\|^{p} .
$$

Therefore in order to show (4.8) it is sufficient to show that $\mathbb{E}\left\|N_{T}^{a, m}-N_{T}^{a}\right\|^{p} \rightarrow 0$ as $m \rightarrow \infty$. This follows directly from the fact that $N^{a}(\omega)$ has independent increments, hence $N^{a, m}(\omega)=\mathbb{E}\left(N^{a}(\omega) \mid \sigma\left(N^{a, m}(\omega)\right)\right)$ due to the construction of $N^{a, m}$, and so the desired holds true by [46, Theorem 3.3.2].

The following theorem sums up Theorem 4.2, Corollary 4.4, and Theorem 4.5.

Theorem 4.6. Let $X$ be a Banach space with the decoupling property, $M$ : $\mathbb{R}_{+} \times \Omega \rightarrow X$ be a local martingale. Assume additionally that $M$ has the canonical decomposition $M=M^{c}+M^{q}+M^{a}$. Assume that $M^{c}, M^{q}$, and $M^{a}$ satisfy the conditions of Theorem 4.2, Corollary 4.4, and Theorem 4.5 respectively. Then $M$ has a decoupled tangent local martingale $N$, and for any $1 \leq p<\infty$ one has that

$$
\mathbb{E} \sup _{0 \leq t \leq T}\left\|M_{t}\right\|^{p} \lesssim_{p, X} \mathbb{E}\left\|N_{T}\right\|^{p}, \quad T \geq 0 .
$$

Proof. Existence of a decoupled tangent local martingale $N$ follows directly from Theorem 4.2, Corollary 4.4, and Theorem 4.5. Let $N=N^{c}+N^{q}+N^{a}$ be the canonical decomposition of $N$. Then by (4.3), (4.7), and (4.8), and by a triangle inequality we have that

$$
\mathbb{E} \sup _{0 \leq t \leq T}\left\|M_{t}\right\|^{p} \lesssim_{p, X} \mathbb{E}\left\|N_{T}^{c}\right\|^{p}+\left\|N_{T}^{q}\right\|^{p}+\left\|N_{T}^{a}\right\|^{p}, \quad T \geq 0 .
$$

It remains to show that

$$
\mathbb{E}\left\|N_{T}\right\|^{p} \bar{\sim}_{p} \mathbb{E}\left\|N_{T}^{c}\right\|^{p}+\left\|N_{T}^{q}\right\|^{p}+\left\|N_{T}^{a}\right\|^{p}, \quad T \geq 0,
$$

which follows from the fact that $N_{T}^{c}(\omega), N_{T}^{q}(\omega)$, and $N_{T}^{a}(\omega)$ are independent mean-zero for a.e. $\omega \in \Omega$ due to Definition 3.3 and Theorem 9.2. 


\section{Convex functions with moderate growth}

A function $\phi: \mathbb{R}_{+} \rightarrow \mathbb{R}_{+}$is called to have a moderate growth if there exists $\alpha>0$ such that $\phi(2 x) \leq \alpha \phi(x)$ for any $x \geq 0$. The goal of the present section is to show the following result about tangent martingales and convex functions with moderate growth which extends Theorem 1.1 to more general functions and to continuous-time martingales and also extends [58, Theorem 4.2] to infinite dimensions.

Theorem 5.1. Let $X$ be a Banach space, $\phi: \mathbb{R}_{+} \rightarrow \mathbb{R}_{+}$be a convex function of moderate growth such that $\phi(0)=0$. Then $X$ is $U M D$ if and only if we have that for any tangent local martingales $M, N: \mathbb{R}_{+} \times \Omega \rightarrow X$

$$
\mathbb{E} \phi\left(M^{*}\right) \bar{\sim}_{\phi, X} \mathbb{E} \phi\left(N^{*}\right),
$$

where $M^{*}:=\sup _{t \geq 0}\left\|M_{t}\right\|$ and $N^{*}:=\sup _{t \geq 0}\left\|N_{t}\right\|$.

In order to prove the theorem we will need two components: the canonical decomposition and good- $\lambda$ inequalities for each part of the canonical decomposition. Namely we will use the following lemma proven by Burkholder in [14, Lemma 7.1] (see also [18, pp. 88-90], [15, pp. 1000-1001], and [91, Section 4] for various forms of general good- $\lambda$ inequalities).

Lemma 5.2. Let $f, g: \Omega \rightarrow \mathbb{R}_{+}$be measurable such that for some $\beta>1, \delta>0$, and $\varepsilon>0$

$$
\mathbb{P}(g>\beta \lambda, f \leq \delta \lambda) \leq \varepsilon \mathbb{P}(g>\lambda), \quad \lambda>0 .
$$

Let $\phi: \mathbb{R}_{+} \rightarrow \mathbb{R}_{+}$be a convex function of moderate growth with $\phi(0)=0$. Let $\gamma<\varepsilon^{-1}$ and $\eta$ be such that

$$
\phi(\beta \lambda) \leq \gamma \phi(\lambda), \quad \phi\left(\delta^{-1} \lambda\right) \leq \eta \phi(\lambda), \quad \lambda>0 .
$$

Then

$$
\mathbb{E} \phi(g) \leq \frac{\gamma \eta}{1-\gamma \varepsilon} \mathbb{E} \phi(f)
$$

\subsection{Good-入 inequalities}

Let us start with good- $\lambda$ inequalities for tangent continuous and purely discontinuous quasi-left continuous martingales. The following good- $\lambda$ inequalities for continuous tangent martingales follow from $L^{p}$ estimates (3.5) analogously good- $\lambda$ inequalities presented in [14, Section 8 and 9].

Proposition 5.3. Let $X$ be a UMD Banach space, $M, N: \mathbb{R}_{+} \times \Omega \rightarrow X$ be tangent continuous local martingales. Then we have that for any $1<p<\infty$, $\delta>0$, and $\beta>1$

$$
\mathbb{P}\left(N^{*}>\beta \lambda, M^{*} \leq \delta \lambda\right) \lesssim_{p, X} \frac{\delta^{p}}{(\beta-1)^{p}} \mathbb{P}\left(N^{*}>\lambda\right), \quad \lambda>0,
$$

where $M^{*}:=\sup _{t \geq 0}\left\|M_{t}\right\|$ and $N^{*}:=\sup _{t \geq 0}\left\|N_{t}\right\|$. 
Let us now show good- $\lambda$ inequalities for stochastic integrals with respect to a random measure. First we will need a definition of a conditionally symmetric martingale.

Definition 5.4. Let $X$ be a Banach space. $M: \mathbb{R}_{+} \times \Omega \rightarrow X$ is called conditionally symmetric if $M$ has local characteristics and if $M$ and $-M$ are tangent.

Remark 5.5. Note that in the discrete case, i.e. when we have an X-valued discrete martingale difference sequence $\left(d_{n}\right)_{n \geq 1}$, the latter definition is equivalent to $\mathbb{P}\left(d_{n} \mid \mathcal{F}_{n-1}\right)$ being symmetric a.s. for any $n \geq 1$.

Now let us state and prove the desired good- $\lambda$ inequalities.

Proposition 5.6. Let $X$ be a UMD Banach space, $M$ and $N$ be $X$-valued tangent purely discontinuous quasi-left continuous conditionally symmetric local martingales. Then for any $\delta>0$ and any $\beta>\delta+1$ we have that

$$
\begin{aligned}
\mathbb{P}\left(N^{*}>\beta \lambda, \Delta M^{*} \vee \Delta N^{*} \vee M^{*}\right. & \leq \delta \lambda) \\
& \lesssim_{p, X} \frac{\delta^{p}}{(\beta-\delta-1)^{p}} \mathbb{P}\left(N^{*}>\lambda\right), \quad \lambda>0,
\end{aligned}
$$

where $\Delta M^{*}:=\sup _{t \geq 0}\left\|\Delta M_{t}\right\|, \Delta N^{*}:=\sup _{t \geq 0}\left\|\Delta N_{t}\right\|, M^{*}:=\sup _{t \geq 0}\left\|M_{t}\right\|$, and $N^{*}:=\sup _{t \geq 0}\left\|N_{t}\right\|$.

For the proof we will need the following elementary lemma.

Lemma 5.7. Let $X, M$, and $N$ be as above, $a>0$. Let

$$
\rho:=\inf \left\{t \geq 0:\left\|\Delta M_{t}\right\| \vee\left\|\Delta N_{t}\right\|>a\right\}
$$

be a stopping time. Then $t \mapsto \Delta M_{\rho} \mathbf{1}_{t \geq \rho}$ and $t \mapsto \Delta N_{\rho} \mathbf{1}_{t \geq \rho}$ are local martingales. Moreover, we have that a.s.

$$
M_{t}=\int_{[0, t] \times X} \mathbf{1}_{\|x\| \leq a} x \mathrm{~d} \bar{\mu}^{M}, \quad N_{t}=\int_{[0, t] \times X} \mathbf{1}_{\|x\| \leq a} x \mathrm{~d} \bar{\mu}^{N}, \quad t \in[0, \rho),
$$

where $\mu^{M}$ and $\mu^{N}$ are as defined by (2.14).

Proof. As $M$ and $N$ are conditionally symmetric and tangent, we may set that $\nu=\nu^{M}=\nu^{N}$ is the compensator for both $\mu^{M}$ and $\mu^{N}$, and that $\nu(\cdot \times B)=$ $\nu(\cdot \times-B)$ a.s. for any Borel set $B \in \mathcal{B}(X)$. Now let

$$
\begin{aligned}
& M_{t}^{\prime}=\int_{[0, t] \times X} \mathbf{1}_{\|\cdot\|>a}(x) x \mathbf{1}_{[0, \rho]}(s) \mathrm{d} \bar{\mu}^{M}(s, x), \quad t \geq 0, \\
& N_{t}^{\prime}=\int_{[0, t] \times X} \mathbf{1}_{\|\cdot\|>a}(x) x \mathbf{1}_{[0, \rho]}(s) \mathrm{d} \bar{\mu}^{N}(s, x), \quad t \geq 0 .
\end{aligned}
$$

These processes are local martingales by the fact that $x$ is locally stochastically integrable with respect to $\bar{\mu}^{M}$ and $\bar{\mu}^{N}$ thanks to Theorem 3.30 , therefore $x \mathbf{1}_{A}$ is also locally integrable with respect to $\bar{\mu}^{M}$ for any $A \subset \widetilde{\mathcal{P}}$ by $[119$, Subsection 
7.2] and $\gamma$-domination [47, Theorem 9.4.1]. On the other hand, as $\nu$ is symmetric in $x \in X$ and as the function $1_{\|\cdot\|>a}(x) x \mathbf{1}_{[0, \rho]}(s)$ is antisymmetric in $x \in X$, by the definition of $\rho$ we have that

$$
\begin{aligned}
M_{t}^{\prime}= & \int_{[0, t] \times X} \mathbf{1}_{\|\cdot\|>a}(x) x \mathbf{1}_{[0, \rho]}(s) \mathrm{d} \bar{\mu}^{M}(s, x) \\
= & \int_{[0, t] \times X} \mathbf{1}_{\|\cdot\|>a}(x) x \mathbf{1}_{[0, \rho]}(s) \mathrm{d} \mu^{M}(s, x) \\
& \quad-\int_{[0, t] \times X} \mathbf{1}_{\|\cdot\|>a}(x) x \mathbf{1}_{[0, \rho]}(s) \mathrm{d} \nu(s, x) \\
= & \int_{[0, t] \times X} \mathbf{1}_{\|\cdot\|>a}(x) x \mathbf{1}_{[0, \rho]}(s) \mathrm{d} \mu^{M}(s, x) \\
= & \sum_{0 \leq s \leq t \wedge \rho} \Delta M_{s} \mathbf{1}_{\left\|\Delta M_{s}\right\|>a}=\Delta M_{\rho} \mathbf{1}_{t \geq \rho},
\end{aligned}
$$

so the desired follows for $M$. The same can be done for $N$.

The second part of the lemma follows from the fact that

$$
M=M^{\prime}+\int_{[0, \cdot \times X} \mathbf{1}_{\|x\| \leq a} x \mathrm{~d} \bar{\mu}^{M}, \quad N=N^{\prime}+\int_{[0, \cdot] \times X} \mathbf{1}_{\|x\| \leq a} x \mathrm{~d} \bar{\mu}^{N}
$$

a.s. on $[0, \rho]$ and the fact that by the considerations above a.s.

$$
M_{t}^{\prime}=\Delta M_{\rho} \mathbf{1}_{t \geq \rho}, \quad N_{t}^{\prime}=\Delta N_{\rho} \mathbf{1}_{t \geq \rho}, \quad t \geq 0 .
$$

Proof of Proposition 5.6. The proof is based on approach of Kallenberg [58, pp. 36-39]. Let us define stopping times

$$
\begin{aligned}
\sigma & :=\inf \left\{t \geq 0:\left\|N_{t}\right\|>\lambda\right\} \\
\tau & :=\inf \left\{t \geq 0:\left\|M_{t}\right\|>\delta \lambda\right\} \\
\rho & :=\inf \left\{t \geq 0:\left\|\Delta M_{t}\right\| \vee\left\|\Delta N_{t}\right\|>\delta \lambda\right\}
\end{aligned}
$$

Let $\mu^{M}$ and $\mu^{N}$ be defined by (2.14), $\bar{\mu}^{M}=\mu^{M}-\nu$ and $\bar{\mu}^{N}=\mu^{N}-\nu$ be the corresponding compensated random measures (as $M$ and $N$ are tangent, $\mu^{M}$ and $\mu^{N}$ have the same compensator). Define

$$
\begin{aligned}
& \widehat{M}_{t}:=\int_{[0, t] \times X} \mathbf{1}_{\|x\| \leq \delta \lambda} x \mathbf{1}_{(\tau \wedge \sigma \wedge \rho, \tau \wedge \rho]}(s) \mathrm{d} \bar{\mu}^{M}(s, x), \quad t \geq 0, \\
& \widehat{N}_{t}:=\int_{[0, t] \times X} \mathbf{1}_{\|x\| \leq \delta \lambda} x \mathbf{1}_{(\tau \wedge \sigma \wedge \rho, \tau \wedge \rho]}(s) \mathrm{d} \bar{\mu}^{N}(s, x), \quad t \geq 0 .
\end{aligned}
$$

Note that by Lemma $5.7, \widehat{M}$ coincides with $M-M^{\tau \wedge \sigma \wedge \rho}$ on $[0, \tau \wedge \rho$ ), so by the definition of $\tau$ and $\rho$ we have that $\widehat{M} \leq 2 \delta \lambda$, and thus by the fact that $\widehat{M}$ and $\widehat{N}$ are tangent by Lemma 3.34, so by Corollary 3.33 for any $1<p<\infty$ we have that

$$
\mathbb{E} \sup _{t \geq 0}\left\|\widehat{N}_{t}\right\|^{p} \lesssim_{p, X} \mathbb{E} \sup _{t \geq 0}\left\|\widehat{M}_{t}\right\|^{p} \leq 2^{p} \delta^{p} \lambda^{p} .
$$


Therefore,

$$
\begin{aligned}
\mathbb{P}\left(N^{*}>\beta \lambda, \Delta M^{*} \vee \Delta N^{*} \vee M^{*} \leq \delta \lambda\right) & \leq \mathbb{P}\left(N^{*}>\beta \lambda, \tau=\rho=\infty\right) \\
& \stackrel{(*)}{\leq} \mathbb{P}\left(\widehat{N}^{*}>(\beta-\delta-1) \lambda\right) \\
& =\mathbb{P}\left(\left(\widehat{N}^{*}\right)^{p}>(\beta-\delta-1)^{p} \lambda^{p}\right) \\
& \leq \frac{1}{(\beta-\delta-1)^{p} \lambda^{p}} \mathbb{E}\left(\widehat{N}^{*}\right)^{p} \\
& \stackrel{(* *)}{\lesssim} p, X \frac{1}{(\beta-\delta-1)^{p} \lambda^{p}} \mathbb{E}\left(\widehat{M}^{*}\right)^{p},
\end{aligned}
$$

where $(*)$ follows from the fact that if $\tau=\rho=\infty$, so by Lemma $5.7 \widehat{N}$ coincides with $N-N^{\sigma}$ on $\mathbb{R}_{+}$and $\left\|N_{\sigma}\right\| \leq\left\|N_{\sigma-}\right\|+\left\|\Delta N_{\sigma}\right\| \leq(1+\delta) \lambda$ on $\{\tau=\rho=\infty\}$, while $(* *)$ holds by $(5.2)$. The desired then follows by

$$
\begin{aligned}
\mathbb{E}\left(\widehat{M}^{*}\right)^{p}=\mathbb{E}\left(\widehat{M}^{*}\right)^{p} \boldsymbol{1}_{\sigma \leq \tau \wedge \rho} & \leq \mathbb{E} 2^{p} \delta^{p} \lambda^{p} \boldsymbol{1}_{\sigma<\infty} \\
& =2^{p} \delta^{p} \lambda^{p} \mathbb{P}(\sigma<\infty)=2^{p} \delta^{p} \lambda^{p} \mathbb{P}\left(N^{*}>\lambda\right) .
\end{aligned}
$$

\subsection{Proof of Theorem 5.1}

First we will prove each case of the canonical decomposition separately, and then compile them using the following proposition.

Proposition 5.8. Let $X$ be a UMD Banach space, $\phi: \mathbb{R}_{+} \rightarrow \mathbb{R}_{+}$be convex of moderate growth such that $\phi(0)=0$. Then for any local martingale $M$ : $\mathbb{R}_{+} \times \Omega \rightarrow X$ with the canonical decomposition $M=M^{c}+M^{q}+M^{a}$ we have that

$$
\mathbb{E} \phi\left(M^{*}\right) \bar{\sim}_{\phi, X} \mathbb{E} \phi\left(\left(M^{c}\right)^{*}\right)+\mathbb{E} \phi\left(\left(M^{q}\right)^{*}\right)+\mathbb{E} \phi\left(\left(M^{a}\right)^{*}\right) .
$$

Proof. Inequality $\lesssim_{\phi, X}$ of (5.3) follows from the fact that $M=M^{c}+M^{q}+M^{a}$ a.s., so $M^{*} \leq\left(M^{c}\right)^{*}+\left(M^{q}\right)^{*}+\left(M^{a}\right)^{*}$ a.s., and the fact that $\phi$ has moderate growth, so a.s.

$$
\phi\left(M^{*}\right) \leq \phi\left(\left(M^{c}\right)^{*}+\left(M^{q}\right)^{*}+\left(M^{a}\right)^{*}\right) \bar{\sim}_{\phi} \phi\left(\left(M^{c}\right)^{*}\right)+\phi\left(\left(M^{q}\right)^{*}\right)+\phi\left(\left(M^{a}\right)^{*}\right) .
$$

Let us show $\gtrsim_{\phi, X}$ of (5.3). As $X$ is UMD, each of $M, M^{c}, M^{q}$, and $M^{a}$ has a covariation bilinear form $\llbracket M \rrbracket, \llbracket M^{c} \rrbracket, \llbracket M^{q} \rrbracket$, and $\llbracket M^{a} \rrbracket$ respectively (see Remark 2.13). Moreover, by $[119$, Subsection 7.6$]$ we have that $\llbracket M \rrbracket=\llbracket M^{c} \rrbracket+$ $\llbracket M^{q} \rrbracket+\llbracket M^{a} \rrbracket$ a.s., and thus by $[119$, Subsection 3.2 and Section 5] (see also Remark 2.13) for any $i \in\{c, q, a\}$

$$
\mathbb{E} \phi\left(M^{*}\right) \bar{\sim}_{\phi, X} \mathbb{E} \phi\left(\gamma\left(\llbracket M \rrbracket_{\infty}\right)\right) \geq \mathbb{E} \phi\left(\gamma\left(\llbracket M^{i} \rrbracket_{\infty}\right)\right) \bar{\sim}_{\phi, X} \mathbb{E} \phi\left(\left(M^{i}\right)^{*}\right) .
$$

This terminates the proof.

Fix $\phi: \mathbb{R}_{+} \rightarrow \mathbb{R}_{+}$convex of moderate growth such that $\phi(0)=0$. 
Theorem 5.9. Let $X$ be a UMD Banach space, $\left(d_{n}\right)_{n \geq 1}$ and $\left(e_{n}\right)_{n \geq 1}$ be tangent martingale difference sequences. Then we have that

$$
\mathbb{E} \phi\left(\sup _{n \geq 1}\left\|\sum_{k=1}^{n} d_{n}\right\|\right) \bar{\sim}_{\phi, X} \mathbb{E} \phi\left(\sup _{n \geq 1}\left\|\sum_{k=1}^{n} e_{n}\right\|\right) .
$$

Proof. Let $\left(r_{n}\right)_{n \geq 1}$ be a sequence of independent Rademachers (see Definition 2.1). Then by $[18,(8.22)]$ and by $[119$, Section 2$]$ we have that

$$
\begin{aligned}
& \mathbb{E} \phi\left(\sup _{n \geq 1}\left\|\sum_{k=1}^{n} r_{n} d_{n}\right\|\right) \bar{\sim}_{\phi, X} \mathbb{E} \phi\left(\sup _{n \geq 1}\left\|\sum_{k=1}^{n} d_{n}\right\|\right), \\
& \mathbb{E} \phi\left(\sup _{n \geq 1}\left\|\sum_{k=1}^{n} r_{n} e_{n}\right\|\right) \bar{\sim}_{\phi, X} \mathbb{E} \phi\left(\sup _{n \geq 1}\left\|\sum_{k=1}^{n} e_{n}\right\|\right) .
\end{aligned}
$$

Finally, $\left(r_{n} d_{n}\right)_{n \geq 1}$ and $\left(r_{n} e_{n}\right)_{n \geq 1}$ are tangent martingale difference sequences with respect to an enlarged filtration $\overline{\mathbb{F}}=\left(\overline{\mathcal{F}}_{n}\right)_{n \geq 1}$ which is generated by the original filtration $\left(\mathcal{F}_{n}\right)_{n \geq 1}$ and by Rademachers $\left(d_{n}\right)_{n \geq 1}$ as for any $n \geq 1$ and for any Borel set $A \in \mathcal{B}(X)$

$$
\begin{aligned}
\mathbb{P}\left(r_{n} d_{n} \mid \overline{\mathcal{F}}_{n-1}\right)(A) & =\mathbb{E}\left(\mathbf{1}_{A}\left(r_{n} d_{n}\right) \mid \overline{\mathcal{F}}_{n-1}\right) \\
& \stackrel{(i)}{=} \frac{1}{2} \mathbb{E}\left(\mathbf{1}_{A}\left(d_{n}\right) \mid \overline{\mathcal{F}}_{n-1}\right)+\frac{1}{2} \mathbb{E}\left(\mathbf{1}_{-A}\left(d_{n}\right) \mid \overline{\mathcal{F}}_{n-1}\right) \\
& \stackrel{(i i)}{=} \frac{1}{2} \mathbb{E}\left(\mathbf{1}_{A}\left(d_{n}\right) \mid \mathcal{F}_{n-1}\right)+\frac{1}{2} \mathbb{E}\left(\mathbf{1}_{-A}\left(d_{n}\right) \mid \mathcal{F}_{n-1}\right) \\
& \stackrel{(i i i)}{=} \frac{1}{2} \mathbb{E}\left(\mathbf{1}_{A}\left(e_{n}\right) \mid \mathcal{F}_{n-1}\right)+\frac{1}{2} \mathbb{E}\left(\mathbf{1}_{-A}\left(e_{n}\right) \mid \mathcal{F}_{n-1}\right) \\
& \stackrel{(i v)}{=} \mathbb{P}\left(r_{n} e_{n} \mid \overline{\mathcal{F}}_{n-1}\right)(A),
\end{aligned}
$$

where $(i)$ follows from the fact that $r_{n}$ is independent of $d_{n}$ and $\overline{\mathcal{F}}_{n-1},(i i)$ follows from the fact that $d_{n}$ is independent of $\sigma\left(r_{1}, \ldots, r_{n-1}\right),($ iii $)$ holds as $\left(d_{n}\right)_{n \geq 1}$ and $\left(e_{n}\right)_{n \geq 1}$ are tangent, and finally (iv) holds as $(i),(i i)$, and (iii) can analogously be shown for $e_{n}$. Moreover, $r_{n} d_{n}$ and $r_{n} e_{n}$ are conditionally symmetric given $\mathcal{F}_{n-1}$ for any $n \geq 1$, so we have that

$$
\mathbb{E} \phi\left(\sup _{n \geq 1}\left\|\sum_{k=1}^{n} r_{n} d_{n}\right\|\right) \bar{\sim}_{\phi, X} \mathbb{E} \phi\left(\sup _{n \geq 1}\left\|\sum_{k=1}^{n} r_{n} e_{n}\right\|\right)
$$

by [44] (see [25, pp. 424-425]). The desired follows from (5.4) and (5.6).

Theorem 5.10. Let $X$ be a UMD Banach space, $M, N: \mathbb{R}_{+} \times \Omega \rightarrow X$ be tangent continuous local martingales. Then $\mathbb{E} \phi\left(M^{*}\right) \bar{\sim}_{\phi, X} \mathbb{E} \phi\left(N^{*}\right)$.

Proof. The proof follows directly from Lemma 5.2 and Proposition 5.3.

Theorem 5.11. Let $X$ be a UMD Banach space, $M, N: \mathbb{R}_{+} \times \Omega \rightarrow X$ be tangent purely discontinuous quasi-left continuous local martingales. Then $\mathbb{E} \phi\left(M^{*}\right) \bar{\sim}_{\phi, X} \mathbb{E} \phi\left(N^{*}\right)$. 
For the proof of the theorem we will need the following lemma.

Lemma 5.12. Let $X$ be a Banach space, $M, N: \mathbb{R}_{+} \times \Omega \rightarrow X$ be tangent local martingales. Then

$$
\mathbb{P}\left(\Delta N^{*} \geq \lambda\right) \leq 6 \mathbb{P}\left(\Delta M^{*} \geq \lambda\right), \quad \lambda>0,
$$

where we set $\Delta M^{*}:=\sup _{t \geq 0}\left\|\Delta M_{t}\right\|$ and $\Delta N^{*}:=\sup _{t \geq 0}\left\|\Delta N_{t}\right\|$.

Proof. By a standard restriction to finite dimensions argument (see e.g. the proof of [115, Theorem 3.3]) and by the fact that $A M$ and $A N$ ate tangent for any linear operator $A \in \mathcal{L}(X, Y)$ (see Theorem A.1) we may assume that $X$ is finite dimensional. Due to Theorem 3.9 we may assume that both $M$ and $N$ are purely discontinuous. Let $M=M^{q}+M^{a}$ and $N=N^{q}+N^{a}$ be the canonical decompositions of $M$ and $N$. Then by (2.8) we have that a.s.

$$
\begin{gathered}
\left\{t \geq 0: \Delta M_{t} \neq 0\right\}=\left\{t \geq 0: \Delta M_{t}^{q} \neq 0\right\} \cup\left\{t \geq 0: \Delta M_{t}^{a} \neq 0\right\}, \\
\left\{t \geq 0: \Delta N_{t} \neq 0\right\}=\left\{t \geq 0: \Delta N_{t}^{q} \neq 0\right\} \cup\left\{t \geq 0: \Delta N_{t}^{a} \neq 0\right\},
\end{gathered}
$$

and

$$
\begin{aligned}
& \left\{t \geq 0: \Delta M_{t}^{q} \neq 0\right\} \cap\left\{t \geq 0: \Delta M_{t}^{a} \neq 0\right\}=\varnothing, \\
& \left\{t \geq 0: \Delta N_{t}^{q} \neq 0\right\} \cap\left\{t \geq 0: \Delta N_{t}^{a} \neq 0\right\}=\varnothing .
\end{aligned}
$$

Thus in order to show (5.7) it is sufficient to prove that

$$
\begin{array}{ll}
\mathbb{P}\left(\left(\Delta N^{q}\right)^{*} \geq \lambda\right) \leq 4 \mathbb{P}\left(\left(\Delta M^{q}\right)^{*} \geq \lambda\right), & \lambda>0, \\
\mathbb{P}\left(\left(\Delta N^{a}\right)^{*} \geq \lambda\right) \leq 2 \mathbb{P}\left(\left(\Delta M^{a}\right)^{*} \geq \lambda\right), \quad \lambda>0 .
\end{array}
$$

First notice that (5.9) follows from a standard discrete approximation of purely discontinuous martingales with accessible jumps (see e.g. the proof of Proposition 3.37 and Subsection B.1) and [29, Lemma 2.3.3]. Let us show (5.8). Without loss of generality we may assume that $M+M^{T}$ and $N=N^{T}$ for some foxed $T>0$. Let us define for any $n \geq 1$

$$
d_{n}^{k}:=M_{T k / n}^{q}-M_{T(k-1) / n}^{q}, \quad e_{n}^{k}:=N_{T k / n}^{q}-N_{T(k-1) / n}^{q}, \quad k=1, \ldots, n .
$$

For each $n \geq 1$, let $\left(\tilde{d}_{n}^{k}\right)_{k=1}^{n}$ be a decoupled tangent sequence of $\left(d_{n}^{k}\right)_{k=1}^{n}$ and $\left(\tilde{e}_{n}^{k}\right)_{k=1}^{n}$ be a decoupled tangent sequence of $\left(e_{n}^{k}\right)_{k=1}^{n}$. Then by [29, Lemma 2.3.3] we have that

$$
\begin{array}{ll}
\mathbb{P}\left(\sup _{k=1}^{n}\left\|e_{k}^{n}\right\| \geq \lambda\right) \leq 2 \mathbb{P}\left(\sup _{k=1}^{n}\left\|\tilde{e}_{k}^{n}\right\| \geq \lambda\right), & \lambda>0, \\
\mathbb{P}\left(\sup _{k=1}^{n}\left\|\tilde{d}_{k}^{n}\right\| \geq \lambda\right) \leq 2 \mathbb{P}\left(\sup _{k=1}^{n}\left\|d_{k}^{n}\right\| \geq \lambda\right), \quad \lambda>0 .
\end{array}
$$

Let $\widetilde{M}^{q}$ be a local martingale decoupled tangent to both $M^{q}$ and $N^{q}$. As $M^{q}$, $N^{q}$, and $\widetilde{M}^{q}$ have càdlàg trajectories (see Subsection 2.5), we have the following convergences

$$
\mathbb{P}-\lim _{n \rightarrow \infty} \sup _{k=1}^{n}\left\|d_{k}^{n}\right\|=\sup _{0 \leq t \leq T}\left\|\Delta M_{t}^{q}\right\|, \quad \mathbb{P}-\lim _{n \rightarrow \infty} \sup _{k=1}^{n}\left\|e_{k}^{n}\right\|=\sup _{0 \leq t \leq T}\left\|\Delta N_{t}^{q}\right\|,
$$


$\mathbb{P}\left(\sup _{k=1}^{n}\left\|\tilde{d}_{k}^{n}\right\|>\lambda\right), \mathbb{P}\left(\sup _{k=1}^{n}\left\|\tilde{e}_{k}^{n}\right\|>\lambda\right) \rightarrow \mathbb{P}\left(\sup _{0 \leq t \leq T}\left\|\Delta \widetilde{M}_{t}\right\|>\lambda\right), \quad n \rightarrow \infty, \quad \lambda>0$,

where the latter follows from Theorem 10.3; thus by (5.10) we have that

$$
\mathbb{P}\left(\left(\Delta N^{q}\right)^{*} \geq \lambda\right) \leq 2 \mathbb{P}\left(\left(\Delta \widetilde{M}^{q}\right)^{*} \geq \lambda\right) \leq 4 \mathbb{P}\left(\left(\Delta M^{q}\right)^{*} \geq \lambda\right), \quad \lambda>0,
$$

so (5.8) (and consequently (5.7)) follows.

Proof of Theorem 5.11. First we prove the conditional symmetric case, and then the general case.

Step 1: conditionally symmetric case. Let $M$ and $N$ be conditionally symmetric. Then by Lemma 5.2 and Proposition 5.6 we have that

$$
\mathbb{E} \phi\left(N^{*}\right) \lesssim_{\phi, X} \mathbb{E} \phi\left(\Delta M^{*} \vee \Delta N^{*} \vee M^{*}\right) .
$$

As $\phi$ has a moderate growth, we have that

$$
\mathbb{E} \phi\left(\Delta M^{*} \vee \Delta N^{*} \vee M^{*}\right) \bar{\sim}_{\phi} \mathbb{E} \phi\left(\Delta M^{*}\right)+\mathbb{E} \phi\left(\Delta N^{*}\right)+\mathbb{E} \phi\left(M^{*}\right),
$$

where

$$
\mathbb{E} \phi\left(\Delta M^{*}\right) \lesssim_{\phi} \mathbb{E} \phi\left(M^{*}\right)
$$

as $\Delta M^{*} \leq 2 M^{*}$, and

$$
\mathbb{E} \phi\left(\Delta N^{*}\right) \leq 6 \mathbb{E} \phi\left(\Delta M^{*}\right) \lesssim_{\phi} \mathbb{E} \phi\left(M^{*}\right),
$$

by Lemma 5.12, since $\mathbb{E} \phi(\xi)=\int_{\mathbb{R}_{+}} \mathbb{P}(\xi>\lambda) \mathrm{d} \phi(\lambda)$ for any random variable $\xi: \Omega \rightarrow \mathbb{R}_{+}$and since $\phi(0)=0$, and by (5.11). Thus we have that $\mathbb{E} \phi\left(N^{*}\right) \lesssim_{\phi}$ $\mathbb{E} \phi\left(M^{*}\right)$; the converse follows similarly.

Step 2: general case. First of all, it is sufficient to assume that $N$ is a decoupled tangent martingale to $M$. Let $N^{\prime}$ be another decoupled tangent martingale to $M$ conditionally independent of $N$ given $\mathcal{F}$. Then $M-N^{\prime}$ and $N-N^{\prime}$ are tangent martingales which are conditionally symmetric, and thus

$$
\mathbb{E} \phi\left(M^{*}\right) \stackrel{(i)}{\leq} \mathbb{E} \phi\left(\left(M-N^{\prime}\right)^{*}\right) \stackrel{(i i)}{\lesssim} \phi, X \mathbb{E} \phi\left(\left(N-N^{\prime}\right)^{*}\right) \stackrel{(i i i)}{\lesssim_{\phi}} \mathbb{E} \phi\left(N^{*}\right),
$$

where $(i)$ holds by the fact that a conditional expectation is a contraction and by the fact that $\phi$ is convex, (ii) follows from Step 1, and (iii) follows by the fact that $\phi$ is convex of moderate growth and that $N$ and $N^{\prime}$ are conditionally independent given $\mathcal{F}$ and equidistributed.

Let us show that

$$
\mathbb{E} \phi\left(N^{*}\right) \lesssim_{\phi, X} \mathbb{E} \phi\left(M^{*}\right) .
$$

Without loss of generality by the dominated convergence theorem we may assume that $M_{t}=M_{T}$ and $N_{t}=N_{T}$ for some fixed $T>0$ and any $t \geq T$. By Theorem 10.3 there exist pure jump processes $\left(M^{n}\right)_{n \geq 1}$ and $\left(N^{n}\right)_{n \geq 1}$ such that

(A) for each $n \geq 1, M^{n}$ and $N^{n}$ have jumps at $\left\{\frac{T}{n}, \ldots, \frac{T(n-1)}{n}, T\right\}$, 
(B) for each $n \geq 1,\left(M_{T k / n}^{n}-M_{T(k-1) / n}^{n}\right)_{k=1}^{n}$ and $\left(N_{T k / n}^{n}-N_{T(k-1) / n}^{n}\right)_{k=1}^{n}$ are martingale difference sequences with respect to the enlarged filtration $\left(\overline{\mathcal{F}}_{T k / n}\right)_{k=1}^{n}\left(\right.$ which enlarges $\left.\left(\mathcal{F}_{T k / n}\right)_{k=1}^{n}\right)$ such that $\left(N_{T k / n}^{n}-N_{T(k-1) / n}^{n}\right)_{k=1}^{n}$ is a decoupled tangent martingale difference sequence to $\left(M_{T k / n}^{n}-M_{T(k-1) / n}^{n}\right)_{k=1}^{n}$,

(C) $N^{n}$ converges to $N$ in distribution as random variables with values in the Skorokhod space $\mathcal{D}([0, T], X)$ as $n \rightarrow \infty$,

(D) $M^{n}$ converges to $M$ a.s. as $n \rightarrow \infty$, and, moreover, $\left(M^{n}\right)^{*} \nearrow M^{*}$ a.s.

By (B) and Theorem 5.9 we have that

$$
\mathbb{E} \phi\left(\left(M^{n}\right)^{*}\right) \bar{\sim}_{\phi, X} \mathbb{E} \phi\left(\left(N^{n}\right)^{*}\right),
$$

for any $n \geq 1$. On the other hand we have that $\mathbb{E} \phi\left(\left(M^{n}\right)^{*}\right) \nearrow \mathbb{E} \phi\left(M^{*}\right)$ by the dominated convergence theorem and (D). Therefore (5.12) follows from (5.13), (C), and Fatou's lemma.

Remark 5.13. Note that if $M$ and $N$ have predictably bounded jumps, i.e. there exists a predictable increasing process $A: \mathbb{R}_{+} \times \Omega \rightarrow \mathbb{R}_{+}$such that $\left\|\Delta M_{t}\right\|,\left\|\Delta N_{t}\right\| \leq A_{t}$ a.s. for any $t \geq 0$, then there is no need in conditional symmetry in the proof of Proposition 5.6, and hence there is no need in using Section 10 in order to prove Theorem 5.11 (see e.g. the proof of Proposition 3.23).

Let us eventually show Theorem 5.1. By Proposition 5.8 it is sufficient to show that

$$
\begin{aligned}
& \mathbb{E} \phi\left(\left(M^{c}\right)^{*}\right) \bar{\sim}_{\phi, X} \mathbb{E} \phi\left(\left(N^{c}\right)^{*}\right), \\
& \mathbb{E} \phi\left(\left(M^{q}\right)^{*}\right) \bar{\sim}_{\phi, X} \mathbb{E} \phi\left(\left(N^{q}\right)^{*}\right), \\
& \mathbb{E} \phi\left(\left(M^{a}\right)^{*}\right) \bar{\sim}_{\phi, X} \mathbb{E} \phi\left(\left(N^{a}\right)^{*}\right) .
\end{aligned}
$$

The inequality (5.14) follows from Theorem 3.9 and 5.10, (5.15) follows from Theorem 3.9 and 5.11, and finally (5.16) holds by Theorem 3.9 and 5.9, and the approximation argument from the proof of Proposition 3.37 and B.1.

\subsection{Not convex functions}

What is of a big interest is whether it is possible to have an analogue of Theorem 5.1 for a general $\phi$ of moderate growth (e.g. $\phi(t)=\sqrt{t}$ ), as it was done in the conditionally symmetric case in [58, Theorem 4.1]. In our case this is possible due to the following theorem.

Theorem 5.14. Let $X$ be a UMD Banach space, $\phi: \mathbb{R}_{+} \rightarrow \mathbb{R}_{+}$be an increasing function of moderate growth such that $\phi(0)=0$. Then for any tangent conditionally symmetric martingales $M, N: \mathbb{R}_{+} \times \Omega \rightarrow X$ we have that

$$
\mathbb{E} \phi\left(M^{*}\right) \bar{\sim}_{\phi, X} \phi\left(N^{*}\right)
$$


Note that $M$ is conditionally symmetric if and only if $M^{d}$ is conditionally symmetric, where $M=M^{c}+M^{d}$ is the Meyer-Yoeurp decomposition of $M$ (see Remark 2.19).

Proof of Theorem 5.14. First note that one can show Proposition 5.6 and Lemma 5.7 for general conditionally symmetric $M$ and $N$ (with modifying $\widehat{M}$ and $\widehat{N}$ by adding to them $M_{\tau \wedge \rho \wedge t}^{c}-M_{\tau \wedge \sigma \wedge \rho}^{c}$ and $N_{\tau \wedge \rho \wedge t}^{c}-N_{\tau \wedge \sigma \wedge \rho}^{c}$ respectively). Then thanks to (5.1) we get that for any $\lambda>0, \delta>0$, and $\beta>1+\delta$

$$
\begin{aligned}
& \mathbb{P}\left(N^{*}>\beta \lambda\right)-\mathbb{P}\left(\Delta M^{*} \vee \Delta N^{*} \vee M^{*}>\delta \lambda\right) \\
& \quad \leq \mathbb{P}\left(N^{*}>\beta \lambda, \Delta M^{*} \vee \Delta N^{*} \vee M^{*} \leq \delta \lambda\right) \lesssim_{p, X} \frac{\delta^{p}}{(\beta-\delta-1)^{p}} \mathbb{P}\left(N^{*}>\lambda\right),
\end{aligned}
$$

so by fixing $p \geq 1, \delta=2$, and $\beta>4$ we derive for some fixed $C_{p, X}>0$ by (5.7)

$$
\begin{aligned}
\mathbb{P}\left(N^{*}>\beta \lambda\right) \leq C_{p, X} \frac{2}{(\beta-3)^{p}} \mathbb{P}\left(N^{*}>\lambda\right) \\
\quad+\mathbb{P}\left(\Delta M^{*}>2 \lambda\right)+\mathbb{P}\left(\Delta N^{*}>2 \lambda\right)+\mathbb{P}\left(M^{*}>2 \lambda\right) \\
\leq C_{p, X} \frac{2}{(\beta-3)^{p}} \mathbb{P}\left(N^{*}>\lambda\right)+8 \mathbb{P}\left(M^{*}>\lambda\right) .
\end{aligned}
$$

Then

$$
\mathbb{E} \phi\left(N^{*}\right)=\int_{\mathbb{R}_{+}} \mathbb{P}\left(\phi\left(N^{*}\right)>\lambda\right) \mathrm{d} \lambda=\int_{\mathbb{R}_{+}} \mathbb{P}\left(N^{*}>\lambda\right)\left(\phi^{-1}\right)^{\prime}(\lambda) \mathrm{d} \lambda,
$$

consequently in particular if $\phi(e x) \leq \alpha \phi(x)$ for some $\alpha>1$ (hence $\phi(\beta x) \leq$ $\beta^{\ln \alpha+1} \phi(x)$ for $\beta$ big enough), which holds as $\phi$ is of moderate growth, then analogously to [58, p. 38] by using the fact that $\phi$ is increasing so $\left(\phi^{-1}\right)^{\prime}$ is nonnegative a.s. on $\mathbb{R}_{+}$

$$
\begin{aligned}
\left(\beta^{-\ln \alpha-1}\right. & \left.-C_{p, X} \frac{2}{(\beta-3)^{p}}\right) \mathbb{E} \phi\left(N^{*}\right) \leq E \phi\left(N^{*} / \beta\right)-C_{p, X} \frac{2}{(\beta-3)^{p}} \mathbb{E} \phi\left(N^{*}\right) \\
& =\int_{\mathbb{R}_{+}}\left(\mathbb{P}\left(N^{*} / \beta>\lambda\right)-C_{p, X} \frac{2}{(\beta-3)^{p}} \mathbb{P}\left(N^{*}>\lambda\right)\right)\left(\phi^{-1}\right)^{\prime}(\lambda) \mathrm{d} \lambda \\
& \leq \int_{\mathbb{R}_{+}} \mathbb{P}\left(M^{*}>\lambda\right)\left(\phi^{-1}\right)^{\prime}(\lambda) \mathrm{d} \lambda=\mathbb{E} \phi\left(M^{*}\right),
\end{aligned}
$$

so the desired follows by choosing $p>\ln \alpha+2$ and $\beta$ big enough.

Unfortunately the author does not know whether Theorem 5.14 holds for general martingales. Nonetheless, the following upper estimate can be shown.

Theorem 5.15. Let $X$ be a UMD Banach space, $\phi: \mathbb{R}_{+} \rightarrow \mathbb{R}_{+}$be an increasing function of moderate growth such that $\phi(0)=0$. Then for any local martingale $M: \mathbb{R}_{+} \times \Omega \rightarrow X$ with a decoupled tangent local martingale $N$ we have that

$$
\mathbb{E} \phi\left(M^{*}\right) \lesssim_{\phi, X} \mathbb{E} \phi\left(N^{*}\right) .
$$


Proof. Let $\widetilde{N}$ be another decoupled tangent local martingale to $M$ which is conditionally independent of $N$ given the local characteristics of $M$. Then by Theorem 5.14 we have that

$$
\mathbb{E} \phi\left((M-\widetilde{N})^{*}\right) \bar{\sim}_{\phi, X} \mathbb{E} \phi\left((N-\widetilde{N})^{*}\right) .
$$

It remains to notice that $\mathbb{P}\left(M^{*} \geq \lambda\right) \leq \mathbb{P}\left((M-\tilde{N})^{*} \geq \lambda / 2\right)+\mathbb{P}\left((\tilde{N})^{*} \geq \lambda / 2\right)$, so $\mathbb{E} \phi\left(M^{*}\right) \lesssim_{\phi} \mathbb{E} \phi\left((M-\widetilde{N})^{*}\right)+\mathbb{E} \phi\left(N^{*}\right)$ by (5.17), the fact that $\phi$ has a moderate growth, and due to the equidistribution of $N$ and $\widetilde{N}$, and to note that by the fact that $N-\widetilde{N}$ has increments which are independent symmetric given the local characteristics of $M$ we have that thanks to (5.17) and [47, Proposition $6.1 .12]$

$$
\mathbb{E} \phi\left((N-\tilde{N})^{*}\right) \approx \mathbb{E} \phi\left(\left\|N_{\infty}-\widetilde{N}_{\infty}\right\|\right) \stackrel{(*)}{\lesssim}{ }_{\phi} \mathbb{E} \phi\left(\left\|N_{\infty}\right\|\right) \lesssim \mathbb{E} \phi\left(N_{\infty}^{*}\right)
$$

where $(*)$ follows from $(5.17)$, the fact that

$$
\mathbb{P}\left(\left\|N_{\infty}-\widetilde{N}_{\infty}\right\|>\lambda\right) \leq \mathbb{P}\left(\left\|N_{\infty}\right\|>\lambda / 2\right)+\mathbb{P}\left(\left\|\widetilde{N}_{\infty}\right\|>\lambda / 2\right),
$$

and the fact that $\phi$ has a moderate growth.

\section{Integration with respect to a general martingale}

The present section is devoted to new estimates for stochastic integrals with values in UMD Banach spaces. These are so-called predictable estimates as we will have a predictable process on the right-hand side since this process depends only on the corresponding local characteristics and thus it is predictable. In particular, these estimates extend sharp bounds for a stochastic integral with respect to a cylindrical Brownian motion obtained by van Neerven, Veraar, and Weis in $[81,82]$ (see also $[106,108]$ for continuous martingale case). On the other hand, this section in some sense extends a recent work [32] by Dirksen and the author on stochastic integration in $L^{q}$-spaces, though the latter publication provides precise formulas for the right-hand side of (6.1), i.e. formulas that do not depend on the decoupled tangent martingale or the corresponding Cox process, but only on $\nu^{M}$. We also wish to note that the obtained below estimates are very different from those proven in [119, Subsection 7.1]: estimates (6.1) are more in the spirit of works of Novikov [84], Burkholder [14], and Rosenthal [99], while [119, Subsection 7.1] is based on Burkholder-Davis-Gundy inequalities, which are similar to square function estimates (see e.g. also [109]).

Theorem 6.1. Let $H$ be a Hilbert space, $X$ be a UMD Banach space, $\widetilde{M}$ : $\mathbb{R}_{+} \times \Omega \rightarrow H$ be a local martingale. Then for any elementary predictable $\Phi$ : $\mathbb{R}_{+} \times \Omega \rightarrow \mathcal{L}(H, X)$ and for any $1 \leq p<\infty$ one has that

$$
\mathbb{E} \sup _{t \geq 0}\left\|\int_{0}^{t} \Phi \mathrm{d} \widetilde{M}\right\|^{p} \bar{\sim}_{p, X} \mathbb{E}\left\|\Phi q_{\widetilde{M}^{c}}^{1 / 2}\right\|_{\gamma\left(L^{2}\left(\mathbb{R}_{+},\left[M^{c}\right] ; H\right), X\right)}^{p}
$$




$$
\begin{gathered}
+\mathbb{E E}_{\mathrm{Cox}}\left\|\int_{\mathbb{R}_{+} \times H} \Phi(s) h \mathrm{~d} \bar{\mu}_{\mathrm{Cox}}^{\widetilde{M}^{q}}(s, h)\right\|^{p} \\
+\mathbb{E} \mathbb{E}_{\mathrm{CI}}\left\|\sum_{0 \leq t<\infty} \Phi \Delta \widetilde{N}_{t}^{a}\right\|^{p}
\end{gathered}
$$

where $\widetilde{M}=\widetilde{M}^{c}+\widetilde{M}^{q}+\widetilde{M}^{a}$ is the canonical decomposition, $q_{\widetilde{M}^{c}}$ is as defined in Subsection 2.6, $\mu_{\mathrm{Cox}}^{\widetilde{M}^{q}}$ is a Cox process directed by $\nu^{M^{q}}$, and $\widetilde{N}^{a}$ is a decoupled tangent martingale to $\widetilde{M}^{a}$ constructed in Theorem 3.39, while $\mathbb{E}_{\mathrm{Cox}}$ denotes an expectation for a fixed $\omega \in \Omega$ in a probability space generated by $\mu_{\mathrm{Cox}}$, and $\mathbb{E}_{\mathrm{CI}}$ denotes an expectation for a fixed $\omega \in \Omega$ in a probability space generated by $\widetilde{N}^{a}$ (see Subsection 2.2).

Proof. The theorem follows from Theorem 2.18, the continuous case [108, Example 3.19 and Theorem 4.1], Theorem 3.22, and Proposition 3.37.

Remark 6.2. As on both the right- and the left-hand sides of (6.1) we have norms (strictly speaking, seminorms, but we can consider a quotient space and make these expressions norms), analogously to [119, Subsection 7.1] we can extend the definition of a stochastic integral to any strongly predictable $\Phi$ : $\mathbb{R}_{+} \times \Omega \rightarrow X$ with

$$
\begin{array}{r}
\mathbb{E}\left\|\Phi q \widetilde{M}^{c}\right\|_{\gamma\left(L^{2}\left(\mathbb{R}_{+},\left[\widetilde{M}^{c}\right] ; H\right), X\right)}+\mathbb{E}_{\mathrm{Cox}}\left\|\int_{\mathbb{R}_{+} \times H} \Phi(s) h \mathrm{~d} \bar{\mu}_{\mathrm{Cox}} \widetilde{M}^{q}(s, h)\right\| \\
+\mathbb{E}_{\mathrm{EI}}\left\|\sum_{0 \leq t<\infty} \Phi \Delta \widetilde{N}_{t}^{a}\right\|<\infty .
\end{array}
$$

Remark 6.3. Due to standard Lenglart's trick [67, Corollaire II] we can extend the upper bounds of $(6.1)$ to $p \in(0,1)$ in the following way

$$
\begin{gathered}
\mathbb{E} \sup _{t \geq 0}\left\|\int_{0}^{t} \Phi \mathrm{d} \widetilde{M}\right\|^{p} \lesssim p, X \mathbb{E}\left\|\Phi q_{\widetilde{M}^{c}}^{1 / 2}\right\|_{\gamma\left(L^{2}\left(\mathbb{R}_{+},\left[\widetilde{M}^{c}\right] ; H\right), X\right)}^{p} \\
+\mathbb{E}\left(\mathbb{E}_{\operatorname{Cox}}\left\|\int_{\mathbb{R}_{+} \times H} \Phi(s) h \mathrm{~d} \bar{\mu}_{\mathrm{Cox}}^{\widetilde{M}^{q}}(s, h)\right\|\right)^{p} \\
+\mathbb{E}\left(\mathbb{E}_{\mathrm{CI}}\left\|\sum_{0 \leq t<\infty} \Phi \Delta \widetilde{N}_{t}^{a}\right\|\right)^{p}
\end{gathered}
$$

Remark 6.4. Why expressions on the right-hand sides of (6.1) and (6.2) can be useful? First, if one fixes $\omega \in \Omega$, then these expressions become stochastic integrals with respect to martingales with independent increments, which it is easier to work with. Second, if we are in the quasi-left continuous setting (i.e. $M^{a}=0$ and we have only Poisson-like jumps), then we end up with $\gamma$-norms and the norms generated by Cox processes, which might be of $\gamma$-radonifying nature but with the Poisson distribution (see Remark 3.26).

Remark 6.5. Thanks to Theorem 4.6 both (6.2) and the upper bound of (6.1) hold true if $X$ has the decoupling property. 


\section{Weak tangency versus tangency}

The natural question is raising up while working with local characteristics in infinite dimensions: given a Banach space $X$ (perhaps, not UMD) and an $X$-valued martingale $M$. Can we have results of the form (3.3) for more general Banach spaces by using a family of local characteristics $\left(\left[\left\langle M, x^{*}\right\rangle^{c}\right], \nu^{\left\langle M, x^{*}\right\rangle}\right)_{x^{*} \in X^{*}}$ instead of local characteristics discovered in Section 3 (note that the latter even might not exist by Theorem 3.7)? And how do these weak local characteristics correspond to the those defined in Section 3? Let us answer these questions. First we will need the following definitions.

Definition 7.1. Let $X$ be a Banach space, $M: \mathbb{R}_{+} \times \Omega \rightarrow X$ be local martingale. Then the family $\left(\left[\left\langle M, x^{*}\right\rangle^{c}\right], \nu^{\left\langle M, x^{*}\right\rangle}\right)_{x^{*} \in X^{*}}$ is called weak local characteristics.

Definition 7.2. Let $X$ be a Banach space, $M, N: \mathbb{R}_{+} \times \Omega \rightarrow X$. Then $M$ and $N$ are weakly tangent if $\left\langle M, x^{*}\right\rangle$ and $\left\langle N, x^{*}\right\rangle$ are tangent for any $x^{*} \in X^{*}$, i.e. if $M$ and $N$ have the same weak local characteristics.

Here we show that weak tangency coincides with tangency in the UMD case, so this approach cannot extend Theorem 3.7 in the UMD setting.

Theorem 7.3. Let $X$ be a Banach space, $M, N: \mathbb{R}_{+} \times \Omega \rightarrow X$ be local martingales which have local characteristics. Then $M$ and $N$ are tangent if and only if they are weakly tangent.

For the proof we will need the following lemma.

Lemma 7.4. Let $X$ be a Banach space, $M: \mathbb{R}_{+} \times \Omega \rightarrow X$ be a martingale. Assume that $M$ has a covariation bilinear form $\llbracket M \rrbracket$. Then for any $t \geq 0$ we have that

$$
X^{*} \rightarrow L^{0}(\Omega), \quad x^{*} \mapsto \llbracket M \rrbracket_{t}\left(x^{*}, x^{*}\right),
$$

is continuous for $X^{*}$ endowed with the weak $k^{*}$ topology.

Proof. By a stopping time argument and by Remark 2.10 we may assume that $\mathbb{E} \sup _{t \geq 0}\left\|M_{t}\right\|<\infty$. Let $\left(x_{n}^{*}\right)_{n \geq 1}$ be a weak* Cauchy sequence with the limit $x^{*}$. By the definition of weak ${ }^{*}$ convergence we have that $\left\langle x, x_{n}^{*}\right\rangle \rightarrow\left\langle x, x^{*}\right\rangle$ for any $x \in X$. Thus by [56, Theorem 26.6] a.s.

$$
\begin{aligned}
\mid \llbracket M \rrbracket_{t}\left(x^{*}, x^{*}\right)- & \llbracket M \rrbracket_{t}\left(x_{n}^{*}, x_{n}^{*}\right)|=|\left[\left\langle M, x^{*}\right\rangle\right]_{t}-\left[\left\langle M, x_{n}^{*}\right\rangle\right]_{t} \mid \\
=\left|2\left[\left\langle M, x^{*}\right\rangle,\left\langle M, x_{n}^{*}-x^{*}\right\rangle\right]_{t}+\left[\left\langle M, x^{*}-x_{n}^{*}\right\rangle\right]_{t}\right| & \leq 2 \sqrt{\left[\left\langle M, x^{*}\right\rangle\right]_{t}} \sqrt{\left[\left\langle M, x^{*}-x_{n}^{*}\right\rangle\right]_{t}}+\left[\left\langle M, x^{*}-x_{n}^{*}\right\rangle\right]_{t},
\end{aligned}
$$

so we have that

$$
\begin{aligned}
\| \llbracket M \rrbracket_{t}\left(x^{*}, x^{*}\right) & -\llbracket M \rrbracket_{t}\left(x_{n}^{*}, x_{n}^{*}\right) \|_{L^{1 / 2}(\Omega)} \\
& \leq\left\|2 \sqrt{\left[\left\langle M, x^{*}\right\rangle\right]_{t}} \sqrt{\left[\left\langle M, x^{*}-x_{n}^{*}\right\rangle\right]_{t}}+\left[\left\langle M, x^{*}-x_{n}^{*}\right\rangle\right]_{t}\right\|_{L^{1 / 2}(\Omega)} \\
& \lesssim\left\|\sqrt{\left[\left\langle M, x^{*}\right\rangle\right]_{t}} \sqrt{\left[\left\langle M, x^{*}-x_{n}^{*}\right\rangle\right]_{t}}\right\|_{L^{1 / 2}(\Omega)}
\end{aligned}
$$




$$
\begin{aligned}
& +\left\|\left[\left\langle M, x^{*}-x_{n}^{*}\right\rangle\right]_{t}\right\|_{L^{1 / 2}(\Omega)} \\
\leq\left\|\sqrt{\left[\left\langle M, x^{*}\right\rangle\right]_{t}}\right\|_{L^{1 / 2}(\Omega)}^{1 / 2} & \left\|\sqrt{\left[\left\langle M, x^{*}-x_{n}^{*}\right\rangle\right]_{t}}\right\|_{L^{1 / 2}(\Omega)}^{1 / 2} \\
& +\left\|\left[\left\langle M, x^{*}-x_{n}^{*}\right\rangle\right]_{t}\right\|_{L^{1 / 2}(\Omega)},
\end{aligned}
$$

and thus it is enough to show that $\left\|\left[\left\langle M, x^{*}-x_{n}^{*}\right\rangle\right]_{t}\right\|_{L^{1 / 2}(\Omega)} \rightarrow 0$ as $n \rightarrow \infty$, which follows from the fact that by Burkholder-Davis-Gundy inequalities [56, Theorem 26.12]

$$
\left\|\left[\left\langle M, x^{*}-x_{n}^{*}\right\rangle\right]_{t}\right\|_{L^{1 / 2}(\Omega)} \approx\left(\mathbb{E} \sup _{0 \leq s \leq t}\left\|\left\langle M, x^{*}-x_{n}^{*}\right\rangle\right\|\right)^{2} \rightarrow 0, n \rightarrow \infty,
$$

as $\left\langle M, x^{*}-x_{n}^{*}\right\rangle \rightarrow 0$ a.s. and $\left\langle M, x^{*}-x_{n}^{*}\right\rangle$ are uniformly bounded by BanachSteinhaus theorem.

Proof of Theorem 7.3. It is clear that tangency implies weak tangency. Let us show the converse. Assume that $M$ and $N$ are weakly tangent. Let $M=M^{c}+$ $M^{d}$ and $N=N^{c}+N^{d}$ be the Meyer-Yoeurp decompositions (see Remark 2.19; this decomposition exists as $M$ and $N$ have local characteristics). First notice that for any $t \geq 0$ and for any $x^{*} \in X^{*}$ a.s.

$$
\begin{aligned}
\llbracket M^{c} \rrbracket_{t}\left(x^{*}, x^{*}\right)=\left[\left\langle M^{c}, x^{*}\right\rangle\right]_{t} \stackrel{(*)}{=} & {\left[\left\langle M, x^{*}\right\rangle^{c}\right]_{t} } \\
& =\left[\left\langle N, x^{*}\right\rangle^{c}\right]_{t} \stackrel{(*)}{=}\left[\left\langle N^{c}, x^{*}\right\rangle\right]_{t}=\llbracket N^{c} \rrbracket_{t}\left(x^{*}, x^{*}\right),
\end{aligned}
$$

where $(*)$ follows from the fact that $\left\langle M^{c}, x^{*}\right\rangle=\left\langle M, x^{*}\right\rangle^{c}$ and $\left\langle N^{c}, x^{*}\right\rangle=\left\langle N, x^{*}\right\rangle^{c}$ a.s. (see $[32,116,118])$. Therefore $\llbracket M^{c} \rrbracket_{t}\left(x^{*}\right)=\llbracket N^{c} \rrbracket_{t}\left(x^{*}\right)$ for any $x^{*} \in X^{*}$ a.s., so we can set $\llbracket M^{c} \rrbracket_{t}=\llbracket N^{c} \rrbracket_{t}$ a.s. on $\Omega$ as $X$ can be assumed separable by the Pettis measurability theorem [46, Theorem 1.1.20], so $\llbracket M^{c} \rrbracket_{t}$ and $\llbracket N^{c} \rrbracket_{t}$ coincide a.s. on weak* dense subset of $X^{*}$ which can be assumed countable by the sequential Banach-Alaoglu theorem, and thus they coincide on the whole $X^{*}$ by Lemma 7.4.

Now let us show that $\nu^{M}=\nu^{N}$ a.s. Fix Borel sets $B \subset X$ and $A \subset \mathbb{R}_{+}$. It is sufficient to show that a.s.

$$
\nu^{M}(A \times B)=\nu^{N}(A \times B)
$$

As $X$ is separable, the Borel $\sigma$-algebra of $X$ is generated by cylinders (see e.g. $[8$, Section 2.1]), we may assume that $B$ is a cylinder as well, i.e. there exist linear functions $x_{1}^{*}, \ldots, x_{m}^{*} \in X^{*}$ and a Borel set $\widetilde{B} \in \mathbb{R}^{m}$ such that $\mathbf{1}_{B}(x)=\mathbf{1}_{\widetilde{B}}\left(\left\langle x, x_{1}^{*}\right\rangle, \ldots,\left\langle x, x_{m}^{*}\right\rangle\right)$. Let $Y=\operatorname{span}\left(x_{1}^{*}, \ldots, x_{m}^{*}\right),\left(y_{n}\right)_{n \geq 1}$ be a dense sequence of $Y$. Then by the assumption of the theorem there exists $\Omega_{0} \subset \Omega$ of full measure such that on $\Omega_{0}$

$$
\nu^{\left\langle M, y_{n}\right\rangle}=\nu^{\left\langle N, y_{n}\right\rangle}, \quad n \geq 1,
$$

and as $\left(y_{n}\right)_{n \geq 1}$ is dense in $Y$ by a continuity argument we have that on $\Omega_{0}$

$$
\nu^{\langle M, y\rangle}=\nu^{\langle N, y\rangle}, \quad y \in Y .
$$


Let $P: X \rightarrow \mathbb{R}^{m}$ be such that $P x=\left(\left\langle x, x_{1}^{*}\right\rangle, \ldots,\left\langle x, x_{m}^{*}\right\rangle\right) \in \mathbb{R}^{m}$ for any $x \in X$. Then by (7.2), by Lemma A.2, and by the Cramér-Wold theorem (see e.g. [7, Theorem 29.4] and [5]) we have that on $\Omega_{0}$

$$
\nu^{M}(A \times B)=\nu^{P M}(A \times \widetilde{B})=\nu^{P N}(A \times \widetilde{B})=\nu^{N}(A \times B),
$$

and thus (7.1) follows. Consequently, $M$ and $N$ have the same local characteristics, and thus they are tangent.

Assume now that inequalities of the form (3.3) hold for some Banach space $X$ for some $1 \leq p<\infty$ for all weakly tangent martingales. Then in particular for any independent Brownian motions $W$ and $\widetilde{W}$ and for any elementary predictable $\Phi: \mathbb{R}_{+} \times \Omega \rightarrow X$ for martingales $M:=\int \Phi \mathrm{d} W$ and $N:=\int \Phi \mathrm{d} W$ we have that by (2.17) for any $t \geq 0$ a.s.

$$
\llbracket M \rrbracket_{t}\left(x^{*}, x^{*}\right)=\left[\left\langle M, x^{*}\right\rangle\right]_{t}=\int_{0}^{t}\left|\Phi^{*} x^{*}\right|^{2} \mathrm{~d} s=\left[\left\langle N, x^{*}\right\rangle\right]_{t}=\llbracket N \rrbracket_{t}\left(x^{*}, x^{*}\right), \quad t \geq 0,
$$

so $M$ and $N$ are weakly tangent and thus by (3.3)

$$
\mathbb{E} \sup _{t \geq 0}\left\|\int_{0}^{t} \Phi \mathrm{d} W\right\|^{p} \bar{\sim}_{p, X} \mathbb{E} \sup _{t \geq 0}\left\|\int_{0}^{t} \Phi \mathrm{d} \widetilde{W}\right\|^{p},
$$

which implies UMD e.g. by $[24,36,46]$ and by good- $\lambda$ inequalities (5.3).

\section{Decoupled tangent martingales and the recoupling property}

An interesting question is the following. Thanks to Theorem 3.8 we know that for any given UMD space $X$ any $X$-valued local martingale has a decoupled tangent local martingale. Using Section 7 one can try to extend the notion of a decoupled tangent local martingale via exploiting weak local characteristics in Definition 3.3, i.e. for a general Banach space $X$ a local martingale $N$ defined on enlarged probability space and filtration is called decoupled tangent to a local martingale $M$ if $M$ is a local martingale with respect to the enlarged filtration having the same weak local characteristics $\left(\left[\left\langle M, x^{*}\right\rangle^{c}\right], \nu^{\left\langle M, x^{*}\right\rangle}\right)_{x^{*} \in X^{*}}$ so that $N(\omega)$ is a local martingale with independent increments with weak local characteristics $\left(\left[\left\langle M, x^{*}\right\rangle^{c}\right](\omega), \nu^{\left\langle M, x^{*}\right\rangle}(\omega)\right)_{x^{*} \in X^{*}}$ for a.e. $\omega$ from the original probability space. For which Banach space $X$ we can guarantee existence of such an object?

In order to answer this question we need the recoupling property, which is dual to the decoupling property (see Definition 4.1).

Definition 8.1. Let $X$ be a Banach space. Then $X$ is said to have the recoupling property if for some $1 \leq p<\infty$, for any $X$-valued martingale difference sequence $\left(d_{n}\right)_{n \geq 1}$ and for a decoupled tangent martingale difference sequence $\left(e_{n}\right)_{n \geq 1}$ one has that

$$
\mathbb{E}\left\|\sum_{n=1}^{\infty} e_{n}\right\|^{p} \lesssim_{p, X} \mathbb{E} \sup _{N \geq 1}\left\|\sum_{n=1}^{N} d_{n}\right\|^{p}
$$


Let us first show the following elementary proposition demonstrating that we can assume any $1 \leq p<\infty$ in Definition 8.1.

Proposition 8.2. Let $X$ be a Banach space, $\left(d_{n}\right)$ be an arbitrary $X$-valued martingale difference sequence, $\left(e_{n}\right)$ be its decoupled tangent martingale sequence. Then the following are equivalent.

(i) $\mathbb{E}\left\|\sum_{n=1}^{\infty} e_{n}\right\|^{p} \lesssim_{p, X} \mathbb{E} \sup _{N \geq 1}\left\|\sum_{n=1}^{N} d_{n}\right\|^{p}$ for any $1 \leq p<\infty$, any $\left(d_{n}\right)$ and $\left(e_{n}\right)$.

(ii) $\mathbb{E}\left\|\sum_{n=1}^{\infty} e_{n}\right\|^{p} \lesssim_{p, X} \mathbb{E} \sup _{N \geq 1}\left\|\sum_{n=1}^{N} d_{n}\right\|^{p}$ for some $1 \leq p<\infty$, any $\left(d_{n}\right)$ and $\left(e_{n}\right)$.

(iii) $\mathbb{E} \mathbb{E}\left(\left\|\sum_{n=1}^{\infty} e_{n}\right\| \mid \mathcal{F}\right)^{p} \lesssim_{p, X} \mathbb{E} \sup _{N \geq 1}\left\|\sum_{n=1}^{N} d_{n}\right\|^{p}$ for some $1 \leq p<\infty$, any $\left(d_{n}\right)$ and $\left(e_{n}\right)$.

(iv) There exists a constant $C_{X}$ such that if $\mathbb{E}\left(\left\|\sum_{n=1}^{\infty} e_{n}\right\| \mid \mathcal{F}\right)>1$ a.s. then we have that $\mathbb{E} \sup _{N \geq 1}\left\|\sum_{n=1}^{N} d_{n}\right\|>C_{X}$.

(v) There exists a constant $C_{X}$ such that if $\left\|\sum_{n=1}^{\infty} e_{n}\right\|>1$ a.s. then we have that $\mathbb{E} \sup _{N \geq 1}\left\|\sum_{n=1}^{N} d_{n}\right\|>C_{X}$.

Proof. The proof in analogous to the one of [37, Theorem 4.1]. (i) $\Rightarrow($ ii $) \Rightarrow$ (iii) follow for an obvious reason and by Jensen's inequality. (iii) $\Rightarrow$ (iv) follows similarly to [15, p. 999]. (iv) $\Rightarrow(v)$ follows from the fact that if $\left\|\sum_{n=1}^{\infty} e_{n}\right\|>1$ a.s., then $\mathbb{E}\left(\left\|\sum_{n=1}^{\infty} e_{n}\right\| \mid \mathcal{F}\right)>1$ a.s., and thus the desired holds. Now let us show $(v) \Rightarrow(i)$. Fix $1 \leq p<\infty$. Let $G_{p}\left(d_{n}\right):=\mathbb{E}\left(\left\|\sum_{n=1}^{\infty} e_{n}\right\|^{p} \mid \mathcal{F}\right)$. Then

$$
\sup _{\lambda>0} \lambda \mathbb{P}\left(G_{p}\left(d_{n}\right)>\lambda\right) \stackrel{(*)}{\leq} \sup _{\lambda>0} \lambda \mathbb{P}\left(\left\|\sum_{n=1}^{\infty} e_{n}\right\|^{p} \geq \lambda\right) \stackrel{(* *)}{\lesssim}{ }_{p, X} \mathbb{E} \sup _{N \geq 1}\left\|\sum_{n=1}^{N} d_{n}\right\|^{p},
$$

where $(*)$ follows from the fact that averaging operators are contractions on Lorentz spaces (see e.g. [55]) and (**) can be derived from $(v)$ analogously [15, p. 1000]. Now applying [15, pp. 999-1000] one can conclude from (8.2) that

$$
\mathbb{E}\left\|\sum_{n=1}^{\infty} e_{n}\right\|^{p}=\mathbb{E} G_{p}\left(d_{n}\right) \lesssim_{p, X} E \sup _{N \geq 1}\left\|\sum_{n=1}^{N} d_{n}\right\|^{p}
$$

so the proposition follows.

Recall that $X$ is called a $U M D^{+}$Banach space if for some (equivalently, for all) $p \in[1, \infty)$, every martingale difference sequence $\left(d_{n}\right)_{n=1}^{\infty}$ in $L^{p}(\Omega ; X)$, and every independent Rademacher sequence $\left(r_{n}\right)_{n=1}^{\infty}$ one has that

$$
\mathbb{E}\left\|\sum_{n=1}^{\infty} r_{n} d_{n}\right\|^{p} \lesssim p, X \mathbb{E} \sup _{N=1}^{\infty}\left\|\sum_{n=1}^{N} d_{n}\right\|^{p}
$$

(see $[24,26,37,46,107])$. 
Remark 8.3. The recoupling property immediately yields the $U M D^{+}$property for Paley-Walsh and Gaussian martingales (see [46, pp. 498-500], [91, Section 4.2], and [37]) and hence any Banach space $X$ with the recoupling property is supereflexive, has finite cotype (see [37, Theorem 3.2]) and nontrivial type (due to [47, Theorem 7.3.8] and the supereflexivity of X). It remains open whether the recoupling property implies UMD (note that the recoupling property is equivalent to UMD if $X$ is a Banach lattice thanks to [59, Theorem 8.4]; see also the discussion in [38] and [46, Section O]). Nonetheless, one can show that the recoupling property is in fact equivalent to $U M D^{+}$for general martingales, which is in some sense a dual result to [24, Theorem 6.6(iii)].

Proposition 8.4. Let $X$ be a Banach space. Then $X$ has the recoupling property if and only if it is $U M D^{+}$.

Proof. The "only if" part. Assume that $X$ has the recoupling property. Let $\left(d_{n}\right)_{n=1}^{\infty}$ be an $X$-valued martingale difference sequence, $\left(e_{n}\right)_{n=1}^{\infty}$ be a corresponding decoupled tangent sequence on an enlarged filtration. Then by Definition 8.1

$$
\mathbb{E} \sup _{N \geq 1}\left\|\sum_{n=1}^{N} d_{n}-e_{n}\right\|^{p} \lesssim p, X \mathbb{E} \sup _{N \geq 1}\left\|\sum_{n=1}^{N} d_{n}\right\|^{p} .
$$

Note that $\left(d_{n}-e_{n}\right)_{n \geq 1}$ is conditionally symmetric. By an approximation argument (by adding a sequence $\left(\varepsilon x r_{n}^{\prime \prime}\right)$ for some $x \in X$, independent Rademacher sequence $\left(r_{n}^{\prime \prime}\right)$, and some small enough $\varepsilon>0$ ) we may assume that $\mathbb{P}\left(d_{n}-e_{n}=0\right)=$ 0 . Moreover, for the same approximation argument we may assume that there exists $x^{*} \in X^{*}$ such that $\left\langle d_{n}-e_{n}, x^{*}\right\rangle \neq 0$ a.s. Let $r_{n}^{\prime}:=\left\langle d_{n}-e_{n}, x^{*}\right\rangle /\left|\left\langle d_{n}-e_{n}, x^{*}\right\rangle\right|$, $\xi_{n}:=\left(d_{n}-e_{n}\right) / r_{n}^{\prime}$. Let us show that $r_{n}^{\prime}$ is independent of $\sigma\left(\xi_{n}, \mathcal{F}_{n-1}\right)$. Indeed, for any $A \subset X$ with $\left\langle x, x^{*}\right\rangle>0$ for any $x \in A$ and any $B \in \mathcal{F}_{n-1}$ by the conditional symmetry we have that

$$
\begin{aligned}
\mathbb{P}\left(\left\{r_{n}^{\prime}=1\right\} \cap\left\{\xi_{n} \in A\right\} \cap B\right) & =\mathbb{P}\left(\left\{d_{n}-e_{n} \in A\right\} \cap B\right) \\
= & \mathbb{P}\left(\left\{d_{n}-e_{n} \in-A\right\} \cap B\right)=\mathbb{P}\left(\left\{r_{n}^{\prime}=-1\right\} \cap\left\{\xi_{n} \in A\right\} \cap B\right),
\end{aligned}
$$

so

$$
\mathbb{P}\left(\left\{r_{n}^{\prime}= \pm 1\right\} \cap\left\{\xi_{n} \in A\right\} \cap B\right)=\frac{1}{2} \mathbb{P}\left(\left\{\xi_{n} \in A\right\} \cap B\right)=\mathbb{P}\left(r_{n}^{\prime}= \pm 1\right) \mathbb{P}\left(\left\{\xi_{n} \in A\right\} \cap B\right) .
$$

Therefore by setting a new filtration $\left(\mathcal{G}_{n}\right)_{n \geq 1}:=\left(\sigma\left(\mathcal{F}_{n}, \xi_{n+1}\right)\right)_{n \geq 1}$ and noticing that $\left(r_{n}^{\prime} \xi_{n}\right)$ is a martingale difference sequence w.r.t. this filtration we can deduce from (8.1), Example 2.28, and the fact that a product of two Rademachers is a Rademacher that for any independent sequence $\left(r_{n}\right)_{n \geq 1}$ of Rademachers

$$
\begin{aligned}
\mathbb{E}\left\|\sum_{n=1}^{\infty} r_{n}\left(d_{n}-e_{n}\right)\right\|^{p}=\mathbb{E} & \left\|\sum_{n=1}^{\infty} r_{n} r_{n}^{\prime} \xi_{n}\right\|^{p} \\
& \lesssim_{p, X} \mathbb{E} \sup _{N \geq 1}\left\|\sum_{n=1}^{N} r_{n}^{\prime} \xi_{n}\right\|^{p}=\mathbb{E} \sup _{N \geq 1}\left\|\sum_{n=1}^{N} d_{n}-e_{n}\right\|^{p} .
\end{aligned}
$$


Finally, by applying a conditional expectation w.r.t. $\sigma\left(\mathcal{F},\left(r_{n}\right)\right)$ and Jensen's inequality we know that $\mathbb{E}\left\|\sum_{n=1}^{\infty} r_{n} d_{n}\right\|^{p} \leq \mathbb{E}\left\|\sum_{n=1}^{\infty} r_{n}\left(d_{n}-e_{n}\right)\right\|^{p}$. By combining all the inequalities above (8.3) follows.

The "if" part. Let $X$ be $\mathrm{UMD}^{+},\left(d_{n}\right)_{n \geq 1}$ be an $X$-valued martingale difference sequence, $\left(e_{n}\right)_{n \geq 1}$ be a decoupled tangent sequence. Given the $\mathrm{UMD}^{+}$ property we need to show (8.1) for some $p \geq 1$. Without loss of generality we may assume that the filtration is generated by $\left(d_{n}\right)$ and the enlarged filtration is generated by $\left(d_{n}\right)$ and $\left(e_{n}\right)$. First assume that $\left(d_{n}\right)_{n \geq 1}$ is conditionally symmetric. In this case for any Borel $A \subset X$ we have that

$$
\mathbb{P}\left(\mathbf{1}_{A}\left(d_{n}\right) \mid \mathcal{F}_{n-1}\right)=\mathbb{P}\left(\mathbf{1}_{-A}\left(d_{n}\right) \mid \mathcal{F}_{n-1}\right), \quad n \geq 1 .
$$

Let $\left(x_{m}\right)_{m=1}^{M} \in X \backslash\{0\}$ be disjoint and let balls $\left(B_{m}\right)_{m=1}^{M}$ be disjoint with $x_{m} \in B_{m}$ and $\{0\} \notin B_{m}$ so $B_{m} \cap-B_{m}=\varnothing$. Then for any $n \geq 1$ we can approximate $d_{n}$ in $L^{p}$ for any $p<\infty$ by $\sum_{m=1}^{M} x_{m}\left(\mathbf{1}_{B_{m}}\left(d_{n}\right)-\mathbf{1}_{-B_{m}}\left(d_{n}\right)\right)$ by taking big enough set $\left(x_{m}\right)_{m=1}^{M} \in X$ and by considering smaller balls $\left(B_{m}\right)_{m=1}^{M}$. Let $A_{m}^{n}:=\left\{d_{n} \in B_{m}\right\} \cup\left\{d_{n} \in-B_{m}\right\}$ and let $r_{m}^{n}:=\mathbf{1}_{B_{m}}\left(d_{n}\right)-\mathbf{1}_{-B_{m}}\left(d_{n}\right)+$ $r_{m}^{n^{\prime}} \mathbf{1}_{\Omega \backslash A_{m}^{n}}$ for some independent $r_{m}^{n^{\prime}}$ (one may need to enlarge the probability space and filtration). By (8.4) and independence of $\left(r_{m}^{n^{\prime}}\right)$ we conclude that

$$
\begin{array}{r}
\mathbb{P}\left(r_{m}^{n}= \pm 1 \mid \mathcal{F}_{n-1}\right)=\mathbb{P}\left(d_{n} \in \pm B_{m} \mid \mathcal{F}_{n-1}\right)+\mathbb{P}\left(\Omega \backslash A_{m}^{n} \cap\left\{r_{m}^{n^{\prime}}= \pm 1\right\} \mid \mathcal{F}_{n-1}\right) \\
=\frac{1}{2} \mathbb{P}\left(A_{m}^{n} \mid \mathcal{F}_{n-1}\right)+\frac{1}{2} \mathbb{P}\left(\Omega \backslash A_{m}^{n} \mid \mathcal{F}_{n-1}\right)=\frac{1}{2}
\end{array}
$$

so $\left(r_{m}^{n}\right)$ is conditionally independent of $\mathcal{F}_{n-1}$. For the same reason $\left(r_{m}^{n}\right)_{m=1}^{M}$ are independent Rademachers which are mutually independent of $\sigma\left(\mathcal{F}_{n-1}, A_{1}^{n}, \ldots, A_{M}^{n}\right)$. Thus we can approximate $d_{n}$ by $\sum_{m=1}^{M} r_{m}^{n} \mathbf{1}_{A_{m}^{n}}$, so by assuming $d_{n}=\sum_{m=1}^{M} r_{m}^{n} \mathbf{1}_{A_{m}^{n}}$, by (8.3), and by the fact that $A_{m}^{n}$ are disjoint for every $n \geq 1$ (which guarantees that the corresponding suprema coincide) we get

$$
\mathbb{E}\left\|\sum_{n=1}^{\infty} \sum_{m=1}^{M} r_{m}^{n^{\prime \prime}} \mathbf{1}_{A_{m}^{n}}\right\|^{p} \lesssim p, X \mathbb{E} \sup _{N=1}^{\infty}\left\|\sum_{n=1}^{N} \sum_{m=1}^{M} r_{m}^{n} \mathbf{1}_{A_{m}^{n}}\right\|^{p},
$$

where $\left(r_{m}^{n^{\prime \prime}}\right)_{n=1, m=1}^{\infty, M}$ is an independent sequence of Rademachers. For each $n \geq 1$ let $e_{n}:=\sum_{m=1}^{M} r_{m}^{n^{\prime \prime}} \mathbf{1}_{A_{m}^{n}}$ and let us show that $\left(e_{n}\right)_{n \geq 1}$ is a decoupled tangent sequence to $\left(d_{n}\right)_{n \geq 1}$. First both $\left(d_{n}\right)$ and $\left(e_{n}\right)$ take values only in $\left( \pm x_{m}\right)_{m=1}^{M}$, and for any $m=1, \ldots, M$ by the independence of $r_{m}^{n^{\prime \prime}}$ and by (8.4)

$$
\begin{aligned}
\mathbb{P}\left(e_{n}= \pm x_{m} \mid \mathcal{F}_{n-1}\right)= & \mathbb{P}\left(\left\{r_{m}^{n^{\prime \prime}}= \pm 1\right\} \cap A_{m}^{n} \mid \mathcal{F}_{n-1}\right)=\frac{1}{2} \mathbb{P}\left(A_{m}^{n} \mid \mathcal{F}_{n-1}\right) \\
& =\mathbb{P}\left(\left\{r_{m}^{n}= \pm 1\right\} \cap A_{m}^{n} \mid \mathcal{F}_{n-1}\right)=\mathbb{P}\left(d_{n}= \pm x_{m} \mid \mathcal{F}_{n-1}\right)
\end{aligned}
$$

so $\left(d_{n}\right)$ and $\left(e_{n}\right)$ are tangent. Next, for any fixed $\omega \in \Omega,\left(e_{n}(\omega)\right)$ are independent, hence $\left(e_{n}\right)$ is decoupled. Thus (8.1) follows from (8.5). 
Now let $\left(d_{n}\right)$ be general. Then for any sequence of independent Rademachers $\left(r_{n}\right)$ by $(8.3)$ and [47, Proposition 6.1.12]

$$
\mathbb{E} \sup _{N=1}^{\infty}\left\|\sum_{n=1}^{N} r_{n} d_{n}\right\|^{p} \approx \mathbb{E}\left\|\sum_{n=1}^{\infty} r_{n} d_{n}\right\|^{p} \lesssim p, X \mathbb{E} \sup _{N=1}^{\infty}\left\|\sum_{n=1}^{N} d_{n}\right\|^{p} .
$$

Let $\left(e_{n}\right)$ be a decoupled tangent sequence to $\left(d_{n}\right)$. Then $\left(r_{n} e_{n}\right)$ is a decoupled tangent sequence to $\left(r_{n} d_{n}\right)$ (see (5.5)), which is conditionally symmetric, and hence by the conditional symmetric case we get

$$
\mathbb{E}\left\|\sum_{n=1}^{\infty} r_{n} e_{n}\right\|^{p} \lesssim p, X \mathbb{E} \sup _{N=1}^{\infty}\left\|\sum_{n=1}^{N} r_{n} d_{n}\right\|^{p} .
$$

It remains to show that $\mathbb{E}\left\|\sum_{n=1}^{\infty} e_{n}\right\|^{p} \bar{\sim}_{p} \mathbb{E}\left\|\sum_{n=1}^{\infty} r_{n} e_{n}\right\|^{p}$, which follows directly from the conditional independence and a randomization argument (see e.g. [66, Lemma 6.3].

Remark 8.5. The same proof yields that the $\mathrm{UMD}^{-}$property (the property which is inverse to $U M D^{+}$, see e.g. [46, Chapter 4]) implies the decoupling property. The converse statement can be shown for conditionally symmetric martingale difference sequences (which is a weaker form of [24, Theorem 6.6(iii)]), but unfortunately a similar technique seems to be not able to provide an extension to general martingales as $U M D^{-}$constants heavily dominate the decoupling constants in the real-valued case (see the discussion in [26, pp. 346-348]). The equivalence of $U M D^{-}$and decoupling remains unknown for the author.

The following theorem is the main result of the section.

Theorem 8.6. Let $X$ be a Banach space. Then the following are equivalent:

(i) $X$ has the recoupling property.

(ii) Any $X$-valued martingale $M$ has a decoupled tangent local martingale $N$.

Moreover, if this is the case, then for any $1 \leq p<\infty$

$$
\mathbb{E} \sup _{t \geq 0}\left\|N_{t}\right\|^{p} \lesssim p, X \mathbb{E} \sup _{t \geq 0}\left\|M_{t}\right\|^{p} .
$$

Proof. Part 1: $(i) \Rightarrow(i i)$. Assume that $X$ has the recoupling property. Let $M$ be an $X$-valued martingale. First let us show that $\llbracket M \rrbracket$ exists, càdlàg, and $\gamma\left(\llbracket M \rrbracket_{t}\right)<\infty$ a.s. for any $t \geq 0$ (see Remark 2.13 and [119]). To this end note that by Proposition 8.4 one can repeat the proof of [119, Theorem 2.1] showing only the upper estimate $\gtrsim_{p, X}$ in [119, (2.2) and (2.3)]. This upper estimate together with the superreflexivity of $X$ (see Remark 8.3) is enough to repeat the proof of [119, Theorem 5.1] again with providing only

$$
\mathbb{E} \gamma\left(\llbracket M \rrbracket_{\infty}\right)^{p} \lesssim_{p, X} \mathbb{E} \sup _{t}\left\|M_{t}\right\|^{p}, \quad 1 \leq p<\infty,
$$


in $[119,(5.1)]$. For the same reason it follows that $\gamma\left(\llbracket M \rrbracket_{t}\right)<\infty$ a.s. for any $t \geq 0$ and that it is locally integrable. Existence of a càdlàg version of $\llbracket M \rrbracket$ holds similarly [119, Proposition 5.5].

Now, as $\llbracket M \rrbracket$ is càdlàg and nondecreasing (see Subsection 2.6 and [119]), analogously to $[56$, Section 25,26$]$ it has a continuous part $\llbracket M \rrbracket^{c}$ and a pure jump part $\llbracket M \rrbracket^{d}$ (shortly, one can simply define $\llbracket M \rrbracket_{t}^{d}:=\sum_{0 \leq s \leq t} \Delta \llbracket M \rrbracket_{s}$ and $\left.\llbracket M \rrbracket^{c}:=\llbracket M \rrbracket-\llbracket M \rrbracket^{d}\right)$. Moreover, by [119, Lemma 3.9, 3.10], by the definition of $\llbracket M \rrbracket$, and by $\left[56\right.$, Theorem 26.14] we have that $\gamma(\llbracket M \rrbracket) \approx \gamma\left(\llbracket M \rrbracket^{c}\right)+\gamma\left(\llbracket M \rrbracket^{d}\right)$ a.s., so in particular by $[119,(3.2)] \llbracket M \rrbracket^{c}$ is bounded and locally integrable.

Let us show that there exists a decoupled tangent martingale $N$. We will construct separately $N^{c}, N^{q}$, and $N^{a}$, and then sum them up. For $N^{c}$ let us consider $\llbracket M \rrbracket^{c}$. We know that $\gamma\left(\llbracket M \rrbracket^{c}\right)$ is finite and locally integrable. Therefore analogously to the proof of Theorem 3.18 there a.s. exist an invertible time-change $\left(\tau_{s}\right)_{s \geq 0}$ with an inverse time-change $\left(A_{t}\right)_{t \geq 0}$ (see the proof of Theorem 3.18), a Hilbert space $H, \Phi \in \gamma\left(L^{2}\left(\mathbb{R}_{+} ; H\right), X\right)$ predictable with respect to $\left(\mathcal{F}_{\tau_{s}}\right)_{s \geq 0}$, and a cylindrical Wiener process $W_{H}$ such that for any $x^{*} \in X^{*}$ a.s.

$$
\left(\left\langle M, x^{*}\right\rangle^{c} \circ \tau\right)_{t}=\int_{0}^{t} \Phi^{*} x^{*} \mathrm{~d} W_{H}, \quad t \geq 0 .
$$

As $\Phi \in \gamma\left(L^{2}\left(\mathbb{R}_{+} ; H\right), X\right)$, by [119, Subsection 3.2] (see also [81]) $\Phi$ is a.s. integrable with respect to an independent cylindrical Brownian motion $W_{H}^{\prime}$ (with $\left(\mathbb{E}_{W_{H}^{\prime}}\left\|\Phi \cdot W_{H}^{\prime}\right\|^{2}\right)^{\frac{1}{2}}=\|\Phi\|_{\gamma\left(L^{2}\left(\mathbb{R}_{+} ; H\right), X\right)}$ a.s. $)$, so we can define $N_{t}^{c}:=\int_{0}^{A_{t}} \Phi \mathrm{d} W_{H}^{\prime}$. Moreover, by (8.7), Remark 8.3, and [119, Lemma 3.10 and Proposition 6.8]

$$
\begin{aligned}
\mathbb{E} \sup _{t \geq 0}\left\|N_{t}^{c}\right\|^{p} & \bar{\sim}_{p, X} \mathbb{E} \gamma\left(\llbracket N^{c} \rrbracket_{\infty}\right)^{p}=\mathbb{E} \gamma\left(\llbracket M^{c} \rrbracket_{\infty}\right)^{p} \\
& \leq \mathbb{E} \gamma\left(\llbracket M \rrbracket_{\infty}\right)^{p} \lesssim_{p, X} \mathbb{E} \sup _{t \geq 0}\left\|M_{t}\right\|^{p} .
\end{aligned}
$$

Let us now construct $N^{q}$. Analogously to [56, Proposition 25.17], $\llbracket M \rrbracket^{d}$ can be decomposed into $\llbracket M \rrbracket^{q}$, which is quasi-left continuous, and $\llbracket M \rrbracket^{a}$, which is purely discontinuous with accessible jumps. Let $\mu^{M}$ be defined by (2.14), let $\mu$ be the quasi-left continuous component of $\mu^{M}$ (see Lemma 3.15), and let $\nu$ be the compensator of $\mu, \bar{\mu}=\mu-\nu$. Then we have that for a.e. $\omega \in \Omega \llbracket M \rrbracket_{\infty}^{q}$ collects quasi-left continuous jumps of $M$ and in particular by [119], $\gamma\left(\llbracket M \rrbracket_{\infty}^{q}\right)=$ $\left(\mathbb{E}_{\gamma}\left\|\int_{\mathbb{R}_{+} \times X} \gamma_{t} x \mathrm{~d} \mu(t, x)\right\|^{2}\right)^{\frac{1}{2}}$, where $\left(\gamma_{t}\right)_{t \geq 0}$ is a family of independent Gaussians which can be considered countable a.s. as $M$ has countably many jumps a.s. Let $\mu_{\text {Cox }}$ be a Cox process directed by $\nu, \bar{\mu}_{\text {Cox }}=\mu_{\text {Cox }}-\nu$. Let us show that $x$ is integrable with respect to $\bar{\mu}_{\mathrm{Cox}}(\omega)$ for a.e. $\omega \in \Omega$. To this end notice that by $[119$, Proposition 6.8 and Subsection 7.2] and by the fact that $\bar{\mu}_{\mathrm{Cox}}(\omega)$ is a Poisson random measure (so it is a random measure with independent increments) $x$ is integrable with respect to $\bar{\mu}_{\text {Cox }}(\omega)$ if $\mathbb{E}_{\text {Cox }}\|x\|_{\gamma\left(L^{2}\left(\mathbb{R}_{+} \times X ; \mu_{\text {Cox }}(\omega)\right), X\right)}<\infty$, which is a.s. satisfied as for any $p \geq 1$

$$
\mathbb{E}\|x\|_{\gamma\left(L^{2}\left(\mathbb{R}_{+} \times X ; \mu_{\text {Cox }}\right), X\right)}^{p} \stackrel{(i)}{=} \mathbb{E}\left(\mathbb{E}_{\gamma}\left\|\int_{\mathbb{R}_{+} \times X} \gamma_{t} x \mathrm{~d} \mu_{\text {Cox }}(\omega)(t, x)\right\|^{2}\right)^{\frac{p}{2}}
$$




$$
\begin{aligned}
& \stackrel{(i i)}{=} \lim _{n \rightarrow \infty} \mathbb{E}\left(\mathbb{E}_{\gamma}\left\|\int_{\mathbb{R}_{+} \times X} \mathbf{1}_{A_{n}}(t, x) \gamma_{n(t)} x \mathrm{~d} \mu_{\operatorname{Cox}}(t, x)\right\|^{2}\right)^{\frac{p}{2}} \\
& \stackrel{(i i i)}{\lesssim} \lim _{p, X} \lim _{n \rightarrow \infty} \mathbb{E}\left(\mathbb{E}_{\gamma}\left\|\int_{\mathbb{R}_{+} \times X} \mathbf{1}_{A_{n}}(t, x) \gamma_{n(t)} x \mathrm{~d} \mu(t, x)\right\|^{2}\right)^{\frac{p}{2}} \\
& \stackrel{(i v)}{=} \mathbb{E}\left(\mathbb{E}_{\gamma}\left\|\int_{\mathbb{R}_{+} \times X} \gamma_{t} x \mathrm{~d} \mu(t, x)\right\|^{2}\right)^{\frac{p}{2}} \\
& \stackrel{(v)}{=} \mathbb{E} \gamma\left(\llbracket M \rrbracket_{\infty}^{q}\right)^{p} \lesssim \mathbb{E} \sup _{t \geq 0}\left\|M_{t}\right\|^{p},
\end{aligned}
$$

where $\left(A_{n}\right)_{n \geq 1}$ are defined analogously to the proof of Theorem 9.2 for quasileft continuous jumps of $M,\left(\gamma_{t}\right)_{t \geq 0}$ are independent Gaussians, a step nondecreasing function $t \mapsto n(t)$ is a discretization so that $\left(\gamma_{n(t)}\right)_{t \geq 0}$ includes finitely many Gaussians and $n(t) \rightarrow t$ as $n \rightarrow \infty$, $(i)$ and $(v)$ follow from [47, Section 9] and the fact that $\mu_{\mathrm{Cox}}$ and $\mu$ are a.s. atomic measures, approximations (ii) and (iv) follow analogously [119, Lemma 3.11], and finally (iii) holds due to (8.1), [66, Lemma 6.3], [47, Proposition 6.3.1], and the fact that $t \mapsto$ $\int_{[0, t] \times X} \mathbf{1}_{A_{n}}(t, x) \gamma_{n(t)} x \mathrm{~d} \mu_{\text {Cox }}(t, x)$ is approximately a decoupled tangent martingale to $t \mapsto \int_{[0, t] \times X} \mathbf{1}_{A_{n}}(t, x) \gamma_{n(t)} x \mathrm{~d} \mu(t, x)$ (in fact $t \mapsto \int_{[0, t] \times X} \gamma_{t} x \mathrm{~d} \mu_{\operatorname{Cox}}(t, x)$ is a decoupled tangent martingale to $t \mapsto \int_{[0, t] \times X} \gamma_{t} x \mathrm{~d} \mu(t, x)$, but the filtration generated by $\left(\gamma_{t}\right)_{t \geq 0}$ is not countably generated, so an approximation needed; such an approximation can be done analogously Section 10). Therefore $t \mapsto N_{t}^{q}:=\int_{[0, t] \times X} x \mathrm{~d} \bar{\mu}_{\text {Cox }}$ is a well-defined purely discontinuous quasi-left continuous martingale which has independent increment for a.e. $\omega \in \Omega$ and thanks to (8.9) and [119, Proposition 6.8 and Subsection 7.2] we have that

$$
\mathbb{E} \sup _{t \geq 0}\left\|N_{t}^{q}\right\|^{p} \bar{\sim}_{p, X} \mathbb{E}\|x\|_{\gamma\left(L^{2}\left(\mathbb{R}_{+} \times X ; \mu_{\text {Cox }}\right), X\right)}^{p} \lesssim_{p, X} \mathbb{E} \sup _{t \geq 0}\left\|M_{t}\right\|^{p}
$$

Finally, let us turn to $N^{a}$. First let $\left(\tau_{n}\right)_{n \geq 1}$ be a sequence of predictable stopping time exhausting predictable jumps of $M$ (equivalently, all the jumps of $\llbracket M \rrbracket^{a}$, see also Lemma 2.20 and [56, Proposition 25.4]). Let $N^{a, m}$ be constructed for any $m \geq 1$ similarly to (3.54). Let us show that $N^{a, m}$ converges in strong $L^{1}(\Omega ; X)$ as $m \rightarrow 0$, i.e. for any $n \geq m \geq 1$

$$
\mathbb{E} \sup _{t \geq 0}\left\|N_{t}^{a, n}-N_{t}^{a, m}\right\| \rightarrow 0, \quad n \geq m \rightarrow \infty .
$$

Note that by its construction $N^{a, n}(\omega)-N^{a, m}(\omega)$ has independent increments, hence by $[119$, Subsection 6.2$]$ and Remark 8.3

$$
\begin{aligned}
\mathbb{E} \sup _{t \geq 0}\left\|N_{t}^{a, n}-N_{t}^{a, m}\right\| & \bar{\sim}_{X} \mathbb{E} \gamma\left(\llbracket N^{a, n}-N^{a, m} \rrbracket_{\infty}\right) \\
& \approx \mathbb{E}\left\|\sum_{k=m+1}^{n} \gamma_{k} \Delta N_{\tau_{k}}\right\|
\end{aligned}
$$




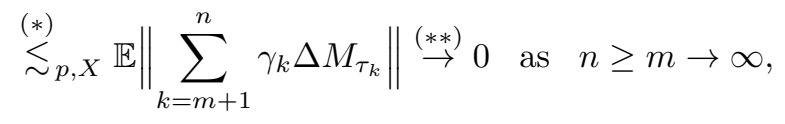

where (*) follows from (8.1), [66, Lemma 6.3], and the fact that $\left(\gamma_{k} \Delta N_{\tau_{k}}\right)_{k=m+1}^{n}$ is a decoupled tangent sequence to $\left(\gamma_{k} \Delta M_{\tau_{k}}\right)_{k=m+1}^{n}$ (this follows analogously Theorem 3.39, where one needs to reorder $\left(\tau_{k}\right)_{k=m+1}^{n}$ making it increasing as it was done in the proof of Proposition 3.37), and (**) holds true similarly to [119, Theorem 7.14]. Thus $N^{a}:=\lim _{n \rightarrow \infty} N^{a, n}$ is a well-defined purely discontinuous martingale with accessible jumps and has independent increments for a.e. fixed $\omega \in \Omega$ due to its construction (see the proof of Theorem 3.39), and analogously $(*)$ in $(8.11)$ (which holds for any power $p \geq 1$ ) and due to [119, Proposition 6.8 and Subsection 7.2] and (8.7)

$$
\mathbb{E} \sup _{t \geq 0}\left\|N_{t}^{a}\right\|^{p} \bar{\sim}_{p, X} \mathbb{E} \gamma\left(\llbracket N^{a} \rrbracket_{\infty}\right)^{p} \lesssim_{p, X} \mathbb{E} \gamma\left(\llbracket M \rrbracket_{\infty}\right)^{p} \lesssim_{p, X} \mathbb{E} \sup _{t \geq 0}\left\|M_{t}\right\|^{p} .
$$

It remains to let $N:=N^{c}+N^{q}+N^{a}$ with (8.6) holding by (8.8), (8.10), and (8.12). The fact that $N$ is a decoupled tangent martingale to $M$ follows similarly Subsection 3.7.

Part 2: $(i i) \Rightarrow(i)$. Let $X$ fail the recoupling property. It is sufficient to show that there exists a martingale without a decoupled tangent local one. First let us construct an $X$-valued martingale difference sequence $\left(d_{n}\right)_{n \geq 1}$ such that $\sum_{n \geq 1} d_{n}$ converges and bounded a.s. and $\sum_{>1} e_{n}$ diverges a.s., where $\left(e_{n}\right)_{n \geq 1}$ is a decoupled tangent sequence to $\left(d_{n}\right)_{n \geq 1}$. To this end we will apply Proposition 8.2. Due to Proposition 8.2(v) for any $m \geq 1$ we can inductively construct an $X$-valued martingale difference sequence $\left(d_{n}^{m}\right)_{m=1}^{M_{m}}$ with a decoupled tangent martingale difference sequence $\left(e_{n}^{m}\right)_{m=1}^{M_{m}}$ such that

(i) $\mathbb{E} \sup _{N=1}^{M_{m}}\left\|\sum_{n=1}^{N} d_{n}^{m}\right\| \leq \frac{1}{2^{m}}$, and

(ii) $\mathbb{P}\left(\left\|\sum_{n=1}^{M_{m}} e_{n}^{m}\right\|>C_{m-1}\right)>1-\frac{1}{2^{m}}$, where $C_{0}=1$ and for any $m \geq 1 C_{m}$ is such a constant bigger then $2 C_{m-1}$ that $\mathbb{P}\left(\left\|\sum_{n=1}^{M_{m}} e_{n}^{m}\right\|>C_{m}\right)<\frac{1}{2^{m}}$

(see e.g. [118, Subsection 4.4]). Then we can set

$$
\begin{array}{ll}
d_{M_{1}+\ldots+M_{m-1}+n}:=d_{n}^{m}, & m \geq 1, \quad 1 \leq n \leq M_{m}, \\
e_{M_{1}+\ldots+M_{m-1}+n}:=e_{n}^{m}, & m \geq 1, \quad 1 \leq n \leq M_{m},
\end{array}
$$

and the pair $\left(d_{n}\right)_{n \geq 1}$ and $\left(e_{n}\right)_{n \geq 1}$ would satisfy the desired properties. Now let us construct a martingale $M: \mathbb{R}_{+} \times \Omega \rightarrow X$ without a decoupled tangent one. To this end let the filtration $\left(\mathcal{F}_{t}\right)_{t \geq 0}$ be generated by $\left(d_{n}\right)_{n \geq 1}$ and $\left(e_{n}\right)_{n \geq 1}$ in the following way: let $\mathcal{F}_{1-1 / 2^{n}}:=\sigma\left(d_{1}, \ldots, d_{n}, e_{1}, \ldots, e_{n}\right)$ and let $\mathcal{F}_{t}:=\mathcal{F}_{1-1 / 2^{n}}$ for any $n \geq 1$ and $t \in\left[1-1 / 2^{n}, 1-1 / 2^{n+1}\right)$. Set

$$
M_{t}:=\sum_{n: 1-1 / 2^{n} \leq t} d_{n}, \quad N_{t}:=\sum_{n: 1-1 / 2^{n} \leq t} e_{n}, \quad 0 \leq t<1 .
$$

First of all notice that $N$ by its definition is a decoupled tangent martingale to $M$ on $\left[0,1-1 / 2^{n}\right]$ for any $n \geq 1$. But $\lim _{t \nearrow_{1}} N_{t}=\sum_{n \geq 1} e_{n}$ does not exists 
due to the construction, so it is not a local martingale and thus not a decoupled tangent local martingale to $M$. Assume that $M$ has some decoupled tangent martingale $\widetilde{N}$. Then by Remark 3.6 for each $\omega \in \Omega$ we have that $\left(\Delta N_{t}(\omega)\right)_{t \geq 0}$ and $\left(\Delta \widetilde{N}_{t}(\omega)\right)_{t \geq 0}$ are equidistributed and independent, so $N$ and $\widetilde{N}$ are equidistributed, hence $\widetilde{N}$ is not a local martingale, so the desired holds true.

Remark 8.7. Note that thanks to the proof of Theorem 8.6 any $U M D^{+}$-valued martingale $M$ has a covariation bilinear form $\llbracket M \rrbracket$, and more importantly, this covariation bilinear form has a continuous part $\llbracket M \rrbracket^{c}$ so that the weak local characteristics of $M$ are generated by $\left(\llbracket M \rrbracket^{c}, \nu^{M}\right)$. It remains unknown for the author whether one can characterize the $U M D^{+}$property of $X$ via existence of the pair $\left(\llbracket M \rrbracket^{c}, \nu^{M}\right)$ (or, equivalently, only via existence of $\llbracket M \rrbracket^{c}$ as $\nu^{M}$ is always well-defined, see Subsection 2.8) for any X-valued martingale $M$.

\section{Independent increments}

The present section is devoted to martingales with independent increments. As we will see below, in this case one could avoid the UMD assumption in order to show existence of local characteristics. Moreover, in Subsection 9.2 we will show that such martingales have a precise form in terms of stochastic integrals with respect to cylindrical Brownian motions and Poisson random measures. Recall that we will be talking about martingales with independent increments without the localization assumption which can be omitted due to Remark 3.5.

\subsection{Weak local characteristics and independent increments}

As it was originally shown by Grigelionis in [42] (see also a multidimensional version [52, p. 106]), a local martingale has independent increments if and only if its local characteristics are deterministic. Let us extend this result to infinite dimensions via using weak local characteristics.

Theorem 9.1. Let $X$ be a Banach space, $M: \mathbb{R}_{+} \times \Omega \rightarrow X$ be a local martingale. Then $M$ has independent increments if and only if its weak local characteristics are deterministic.

Proof. The "only if" part is simple and follows directly from the real-valued case [42] and the fact that if $M$ has independent increments then $\left\langle M, x^{*}\right\rangle$ has independent increments for any $x^{*} \in X^{*}$ as well.

Let us show the "if" part. First we reduct to the finite dimensional case. By the Pettis measurability theorem [46, Theorem 1.1.20] we may assume that $X$ is separable. Let $\left(x_{n}\right)_{n \geq 1}$ be a dense sequence in $X \backslash\{0\},\left(x_{n}^{*}\right)_{n \geq 1}$ be a norming sequence, i.e. $\left\langle x_{n}, x_{n}^{*}\right\rangle=\left\|x_{n}\right\|$ and $\left\|x_{n}^{*}\right\|=1$ for any $n \geq 1$ (such linear functionals exist by the Hahn-Banach theorem). For each $m \geq 1$ define $Y_{m}:=\operatorname{span}\left(x_{1}^{*}, \ldots x_{m}^{*}\right)$ and let $P_{m}: Y_{m} \rightarrow X^{*}$ be the corresponding inclusion operator. Then by the definition of $\left(x_{n}\right)_{n \geq 1}$ and $\left(x_{n}^{*}\right)_{n \geq 1}$ we have that the Borel $\sigma$-algebra of $X$ is generated by $\left(x_{n}^{*}\right)_{n \geq 1}$ (e.g. $x$ in the unit ball of $X$ if and only 
if $\left|\left\langle x, x_{n}^{*}\right\rangle\right| \leq 1$ for all $n \geq 1$ ), and so by the definition of $P_{m}$ we have that $M$ has independent increments if and only if $P_{m}^{*} M$ has independent increments for any $m \geq 1$. So we need to prove the theorem for any $m \geq 1$, which is equivalent to proving it for finite dimensional case as $P_{m}^{*} M$ takes values in a finite dimensional space $\operatorname{ran}\left(P_{m}^{*}\right)$.

Now let $X$ be finite dimensional. Then the theorem follows from [52, Theorem II.4.15].

\subsection{General form of a martingale with independent increments}

Now we are going to show that any martingale with independent increments (with values in any Banach space) has local characteristics, so there is no need in weak local characteristics. Moreover, any such a martingale has a very specific form outlined in Theorem 9.2. Recall that a vector-valued stochastic integral of a deterministic function with respect to a compensated Poisson random measure was defined in Definition 2.24.

Theorem 9.2. Let $X$ and $M$ be as in Theorem 9.1. Assume additionally that $M_{0}=0$. Then $M$ has the canonical decomposition $M=M^{c}+M^{q}+M^{a}$, where martingales $M^{c}, M^{q}$, and $M^{a}$ are independent and have independent increments, and for any $\phi: \mathbb{R}_{+} \rightarrow \mathbb{R}_{+}$with moderate growth and with $\phi(0)=0$ we have that

$$
\mathbb{E} \sup _{t \geq 0} \phi\left(\left\|M_{t}\right\|\right) \bar{\sim}_{\phi} \mathbb{E} \sup _{t \geq 0} \phi\left(\left\|M_{t}^{c}\right\|\right)+\mathbb{E} \sup _{t \geq 0} \phi\left(\left\|M_{t}^{q}\right\|\right)+\mathbb{E} \sup _{t \geq 0} \phi\left(\left\|M_{t}^{a}\right\|\right) .
$$

Moreover, there exist a cylindrical Brownian motion $W_{H}, \Phi \in \gamma\left(L^{2}\left(\mathbb{R}_{+} ; H\right), X\right)$ locally, and a deterministic time-change $\tau^{c}$ such that $M^{c} \circ \tau^{c}=\Phi \cdot W_{H}$, there exists a Poisson random measure $N_{\nu_{n a}}$ on $\mathbb{R}_{+} \times X$ with a compensator $\nu_{n a}$ (which is a non-atomic in time part of $\nu^{M}$ ) such that $M^{q}=\int_{[0, \cdot] \times J} x \mathrm{~d} \widetilde{N}_{\nu_{n a}}$ (where $\tilde{N}_{\nu_{n a}}:=N_{\nu_{n a}}-\nu_{n a}$ ), and finally $M^{a}$ is a martingale which has fixed jump times.

Recall that $\nu_{n a}$ was defined in Lemma 3.15 since a measure is quasi-left continuous if and only if the corresponding compensator is non-atomic in time by Remark 3.16 (see also [57, Theorem 9.22]). In order to prove Theorem 9.2 we will use these lemmas.

Lemma 9.3. Let $X$ be a Banach space, $M: \mathbb{R}_{+} \times \Omega \rightarrow X$ be a martingale with independent increments. Then we have that for any $\phi: \mathbb{R}_{+} \rightarrow \mathbb{R}_{+}$with moderate growth and with $\phi(0)=0$

$$
\mathbb{E} \sup _{0 \leq t \leq T} \phi\left(\left\|M_{t}\right\|\right) \bar{\sim}_{\phi} \mathbb{E} \phi\left(\left\|M_{T}\right\|\right), \quad T \geq 0 .
$$

Proof. Let $\widetilde{M}$ be an independent copy of $M$. Then $M-\widetilde{M}$ is a symmetric 
martingale with independent increments, so by [29, Proposition 1.1.2]

$$
\begin{aligned}
\mathbb{E} \sup _{0 \leq t \leq T} \phi\left(\left\|M_{t}-\widetilde{M}_{t}\right\|\right)=\int_{\lambda>0} \mathbb{P}\left(\sup _{0 \leq t \leq T}\left\|M_{t}-\widetilde{M}_{t}\right\|>\lambda\right) \mathrm{d} \phi(\lambda) \\
\leq 2 \int_{\lambda>0} \mathbb{P}\left(\left\|M_{T}-\widetilde{M}_{T}\right\|>\lambda\right) \mathrm{d} \phi(\lambda)=2 \mathbb{E} \phi\left(\left\|M_{T}-\widetilde{M}_{T}\right\|\right) .
\end{aligned}
$$

Moreover, by conditional Jensen's inequality [46, Proposition 2.6.29], by a triangle inequality, by the fact that $\phi$ has moderate growth, and by the fact that $M$ and $\widetilde{M}$ are equidistributed we have that

$$
\begin{gathered}
\mathbb{E} \sup _{0 \leq t \leq T} \phi\left(\left\|M_{t}-\widetilde{M}_{t}\right\|\right) \bar{\sim}_{\phi} \mathbb{E} \sup _{0 \leq t \leq T} \phi\left(\left\|M_{t}\right\|\right), \\
\mathbb{E} \phi\left(\left\|M_{T}-\widetilde{M}_{T}\right\|\right) \bar{\sim}_{\phi} \mathbb{E} \phi\left(\left\|M_{T}\right\|\right),
\end{gathered}
$$

so (9.2) follows from (9.3) and (9.4).

Lemma 9.4. Let $X$ be a separable Banach space, $\left(x_{n}\right)_{n \geq 1}$ be a dense subset of $X,\left(x_{n}^{*}\right)_{n \geq 1}$ be a norming sequence, i.e. $\left\langle x_{n}, x_{n}^{*}\right\rangle=\left\|x_{n}\right\|$ and $\left\|x_{n}^{*}\right\|=1$ for any $n \geq 1$. Let $M: \mathbb{R}_{+} \times \Omega \rightarrow X$ be a local martingale. Then

(I) $M$ is continuous if and only if $\left\langle M, x_{n}^{*}\right\rangle$ is continuous for any $n \geq 1$,

(II) $M$ is purely discontinuous if and only if $\left\langle M, x_{n}^{*}\right\rangle$ is purely discontinuous for any $n \geq 1$,

(III) $M$ is quasi-left continuous if and only if $\left\langle M, x_{n}^{*}\right\rangle$ is quasi-left continuous for any $n \geq 1$,

(IV) $M$ is with accessible jumps if and only if $\left\langle M, x_{n}^{*}\right\rangle$ is with accessible jumps for any $n \geq 1$.

Proof. The "only if" part of each of the statements is obvious. Let us show the "if" part. First let us start with (I). Assume that $M$ is not continuous. Then there exists a stopping time $\tau$ such that $\mathbb{P}\left(\Delta M_{\tau} \neq 0\right)>0$. Without loss of generality by multiplying $M$ by a constant we may assume that $\mathbb{P}\left(\left\|\Delta M_{\tau}\right\|>\right.$ 1) $>0$. Fix $\varepsilon<1 / 2$. Then, as $\left(x_{n}\right)_{n \geq 1}$ is dense in $X$, there exists $n \geq 1$ such that $\left\|x_{n}\right\|>1-\varepsilon$ and for a ball $B$ with centre in $x_{n}$ and radius $\varepsilon$ we have that $\mathbb{P}\left(\Delta M_{\tau} \in B\right)>0$ (such a ball exists as $X$ can be covered by countably many such balls). Then

$$
\mathbb{P}\left(\left\langle\Delta M_{\tau}, x_{n}^{*}\right\rangle \neq 0\right) \geq \mathbb{P}\left(\left\langle\Delta M_{\tau}, x_{n}^{*}\right\rangle \in\left[\left\|x_{n}\right\|-\varepsilon,\left\|x_{n}\right\|+\varepsilon\right]\right) \geq \mathbb{P}\left(\Delta M_{\tau} \in B\right)>0,
$$

so $\left\langle M, x_{n}^{*}\right\rangle$ is not continuous and the desired follows.

Now let us turn to (II). Assume that $M$ is not purely discontinuous. By [116, Subsection 2.5] (see also [32, Subsection 5.2]) it is analogous to the fact that there exists a continuous uniformly bounded martingale $N: \mathbb{R}_{+} \times \Omega \rightarrow \mathbb{R}$ such that $N_{0}=0$ and $M N$ is not a martingale. Moreover, by exploiting the proof of [116, Proposition 2.10] we even can find such $N$ that $\mathbb{E} M_{t} N_{t} \neq 0$ for some $t \geq 0$. On the other hand if $\left\langle M, x_{n}^{*}\right\rangle$ is purely discontinuous for any $n \geq 1$, then by [116, Proposition 2.10] $\left\langle M, x_{n}^{*}\right\rangle N$ is a martingale starting in zero, so

$$
\left\langle\mathbb{E} M_{t} N_{t}, x_{n}^{*}\right\rangle=\mathbb{E}\left\langle M_{t} N_{t}, x_{n}^{*}\right\rangle=\mathbb{E}\left\langle M_{t}, x_{n}^{*}\right\rangle N_{t}=0,
$$


consequently $\mathbb{E} M_{t} N_{t}=0$ as $\left(x_{n}^{*}\right)_{n \geq 1}$ is a norming sequence, and thus $M$ is purely discontinuous.

Let us show (III). Let $\tau$ be a predictable stopping time. Then it can be shown that $\Delta M_{\tau}=0$ a.s. analogously (I), so $M$ is quasi-left continuous. (IV) follows similarly.

Corollary 9.5. Let $X,\left(x_{n}\right)_{n \geq 1} \in X$ and $\left(x_{n}^{*}\right)_{n \geq 1} \in X^{*}$ be as in Lemma 9.4. Let $M, M^{c}, M^{q}, M^{a}: \mathbb{R}_{+} \times \Omega \rightarrow X$ be local martingales. Then $M=M^{c}+M^{q}+M^{a}$ is the canonical decomposition of $M$ if and only if $\left\langle M, x_{n}^{*}\right\rangle=\left\langle M^{c}, x_{n}^{*}\right\rangle+\left\langle M^{q}, x_{n}^{*}\right\rangle+$ $\left\langle M^{a}, x_{n}^{*}\right\rangle$ is the canonical decomposition of $\left\langle M, x_{n}^{*}\right\rangle$ for any $n \geq 1$.

Eventually we are going to show Theorem 9.2.

Proof of Theorem 9.2. Without loss of generality assume that $M_{0}=0$ a.s. We can also set that there exists $T>0$ such that $M_{t}=M_{T}$ for $t \geq T$, so $\mathbb{E} \sup _{t>0}\left\|M_{t}\right\| \approx \mathbb{E}\left\|M_{T}\right\|<\infty$ (see Remark 3.5 and Lemma 9.3). First of all let us prove the first part of the proposition in the finite dimensional case, and then treat the whole proposition in infinite dimensions.

Step 1. $X$ is finite dimensional. First assume that $X$ is finite dimensional. Then the existence of the canonical decomposition is guaranteed by Theorem 2.18. Let us show that $M^{c}, M^{q}$, and $M^{a}$ are independent and have independent increments. By Proposition $3.10 M^{c}$ has local characteristics $\left(\llbracket M^{c} \rrbracket, 0\right)$. Further, by Lemma 2.22, Proposition 3.11, 3.12 and $3.14 M^{q}$ has local characteristics $\left(0, \nu_{n a}^{M}\right)$, where $\nu_{n a}^{M}$ is the nonatomic part of $\nu^{M}$, and $M^{a}$ has local characteristics $\left(0, \nu_{a}^{M}\right)$, where $\nu_{a}^{M}$ is the atomic part of $\nu^{M}$. Each of three local characteristics are deterministic, so by Theorem 9.1 each of $M^{c}, M^{q}$, and $M^{a}$ has independent increments. Let us show that $M^{c}, M^{q}$, and $M^{a}$ are independent. By the Lévy-Khinchin-type formula [52, II.4.16] (see also Remark 11.4) and by the fact that $M, M^{c}, M^{q}$, and $M^{a}$ have independent increments we have that for any $t_{0}<t_{1}<\ldots<t_{N}$, for any numbers $\left(a_{n}\right)_{n=1}^{N},\left(b_{n}\right)_{n=1}^{N}$, and $\left(c_{n}\right)_{n=1}^{N}$, and for any vectors $\left(x_{n}^{*}\right)_{n=1}^{N},\left(y_{n}^{*}\right)_{n=1}^{N},\left(z_{n}^{*}\right)_{n=1}^{N} \in X^{*}$

$$
\begin{aligned}
\mathbb{E} & \exp \left\{\sum_{n=1}^{N}\left\langle x_{n}^{*}, M_{t_{n}}^{c}-M_{t_{n-1}}^{c}\right\rangle+\left\langle y_{n}^{*}, M_{t_{n}}^{q}-M_{t_{n-1}}^{q}\right\rangle+\left\langle z_{n}^{*}, M_{t_{n}}^{a}-M_{t_{n-1}}^{a}\right\rangle\right\} \\
& \stackrel{(i)}{=} \Pi_{n=1}^{N} \mathbb{E} \exp \left\{\left\langle x_{n}^{*}, M_{t_{n}}^{c}-M_{t_{n-1}}^{c}\right\rangle+\left\langle y_{n}^{*}, M_{t_{n}}^{q}-M_{t_{n-1}}^{q}\right\rangle+\left\langle z_{n}^{*}, M_{t_{n}}^{a}-M_{t_{n-1}}^{a}\right\rangle\right\} \\
& \stackrel{(i i)}{=} \Pi_{n=1}^{N} \mathbb{E} e^{\left\langle x_{n}^{*}, M_{t_{n}}^{c}-M_{t_{n-1}}^{c}\right\rangle} \mathbb{E} e^{\left\langle y_{n}^{*}, M_{t_{n}}^{q}-M_{t_{n-1}}^{q}\right\rangle} \mathbb{E} e^{\left\langle z_{n}^{*}, M_{t_{n}}^{a}-M_{t_{n-1}}^{a}\right\rangle} \\
& \stackrel{(i i i)}{=} \mathbb{E} e^{\sum_{n=1}^{N}\left\langle x_{n}^{*}, M_{t_{n}}^{c}-M_{t_{n-1}}^{c}\right\rangle} \mathbb{E} e^{\sum_{n=1}^{N}\left\langle y_{n}^{*}, M_{t_{n}}^{q}-M_{t_{n-1}}^{q}\right\rangle} \mathbb{E} e^{\sum_{n=1}^{N}\left\langle z_{n}^{*}, M_{t_{n}}^{a}-M_{t_{n-1}}^{a}\right\rangle},
\end{aligned}
$$

where (i) follows from the fact that $M, M^{c}, M^{q}$, and $M^{a}$ have independent increments (so that $M_{t_{n}}^{i}-M_{t_{n-1}}^{i}$ is independent of $M_{t_{m}}^{j}-M_{t_{m-1}}^{j}$ for any $i, j \in$ $\{c, q, a\}, i \neq j$, and any $n \neq m$, which can be shown by the Lévy-Khinchin-type formula [52, II.4.16] and by [103, Theorem II.12.4]), ( $i$ i) follows by [52, II.4.16], and (iii) follows analogously $(i)$. Now the desired independence follows from [103, Theorem II.12.4]. 
Now let us show (9.1). By Lemma 9.3 it is sufficient to show that

$$
\mathbb{E} \phi\left(\left\|M_{t}\right\|\right) \bar{\sim}_{\phi} \mathbb{E} \phi\left(\left\|M_{t}^{c}\right\|\right)+\mathbb{E} \phi\left(\left\|M_{t}^{q}\right\|\right)+\mathbb{E} \phi\left(\left\|M_{t}^{a}\right\|\right), \quad t \geq 0 .
$$

First $M_{t}^{c}, M_{t}^{q}$, and $M_{t}^{a}$ are independent mean zero, so for any $i \in\{c, q, a\}$ we have that $M_{t}^{i}=\mathbb{E}\left(M_{t} \mid \sigma\left(M_{t}^{i}\right)\right)$, consequently by Jensen's inequality

$$
\mathbb{E} \phi\left(\left\|M_{t}^{i}\right\|\right)=\mathbb{E} \phi\left(\left\|\mathbb{E}\left(M_{t} \mid \sigma\left(M_{t}^{i}\right)\right)\right\|\right) \leq \mathbb{E}\left[\mathbb{E}\left(\phi\left(\left\|M_{t}\right\|\right) \mid \sigma\left(M_{t}^{i}\right)\right)\right]=\mathbb{E} \phi\left(\left\|M_{t}\right\|\right),
$$

so $\gtrsim$ in (9.5) follows. On the other hand as $\phi$ has moderate growth and as $M=M^{c}+M^{q}+M^{a}$ we have $\lesssim_{\phi}$ of (9.5).

Step 2. $X$ is general. Now let $X$ be general. We will constrict each part of the canonical decomposition separately and show that each of them has the form predicted in the second part of the theorem.

Step 2. Part 1. Construction of $M^{a}$. Let $\left(t_{m}\right)_{m \geq 1} \subset \mathbb{R}_{+}$be such that $\mathbb{P}\left(\Delta M_{t_{m}} \neq 0\right)>0$ for any $m \geq 1$ (recall that càdlàg processes have at most countably many jumps, so the set of such $t_{m}$ 's is at most countable). For each $t \geq 0$ define

$$
\mathcal{F}^{a}:=\sigma\left(\Delta M_{t_{m}}: m \geq 1\right), \quad M_{t}^{a}:=\mathbb{E}\left(M_{t} \mid \mathcal{F}^{a}\right), \quad t \geq 0 .
$$

Let us show that $M^{a}$ is an $\mathbb{F}$-martingale. Let

$$
\mathcal{F}_{t}^{a}:=\sigma\left(\Delta M_{t_{m}}: 0 \leq t_{m} \leq t\right), \quad \mathcal{F}_{>t}^{a}:=\sigma\left(\Delta M_{t_{m}}: t_{m}>t\right), \quad t \geq 0 .
$$

Then for any $t \geq 0$

$$
\mathbb{E}\left(M_{t} \mid \mathcal{F}^{a}\right) \stackrel{(*)}{=} \mathbb{E}\left(M_{t} \mid \mathcal{F}_{t}^{a} \otimes \mathcal{F}_{>t}^{a}\right) \stackrel{(* *)}{=} \mathbb{E}\left(M_{t} \mid \mathcal{F}_{t}^{a}\right),
$$

where $(*)$ holds from the fact that $\mathcal{F}_{t}^{a}$ and $\mathcal{F}_{>t}^{a}$ are independent, and $(* *)$ holds since $M_{t}$ is independent of $\mathcal{F}_{>t}^{a}$. Therefore $M_{t}^{a}$ is $\mathcal{F}_{t}$-measurable. Now fix $t \geq$ $s \geq 0$. Then $M_{t}-M_{s}$ is independent of $\mathcal{F}_{s}^{a}$ and $\mathcal{F}_{>s}^{a}$ is independent of $\mathcal{F}_{s}$, and thus

$$
\begin{aligned}
\mathbb{E}\left(M_{t}^{a}-M_{s}^{a} \mid \mathcal{F}_{s}\right)=\mathbb{E}\left(\mathbb{E}\left(M_{t}-M_{s} \mid \mathcal{F}_{s}^{a} \otimes \mathcal{F}_{>s}^{a}\right) \mid \mathcal{F}_{s}\right) \\
\quad=\mathbb{E}\left(\mathbb{E}\left(M_{t}-M_{s} \mid \mathcal{F}_{>s}^{a}\right) \mid \mathcal{F}_{s}\right)=\mathbb{E}\left(M_{t}-M_{s}\right)=0,
\end{aligned}
$$

consequently, $M^{a}$ is a martingale. Let us show that $M^{a}$ is a purely discontinuous martingale with accessible jumps. To this end it is sufficient to notice that $M^{a}$ is an $\mathbb{F}^{a}$-martingales (where $\mathbb{F}^{a}=\left(\mathcal{F}_{t}^{a}\right)_{t \geq 0}$ ), so for any $x^{*} \in X^{*}$ a process $t \mapsto\left\langle M_{t}^{a}, x^{*}\right\rangle$ is $\mathbb{F}^{a}$-adapted, and hence it is purely discontinuous with jumps in the set $\left(t_{m}\right)_{m \geq 1}$ because of the structure $\mathbb{F}^{a}$. Indeed, let $L:=\left\langle M^{a}, x^{*}\right\rangle$. For each $k \geq 1$ define

$$
L_{t}^{k}:=\sum_{m \leq k} \mathbb{E}\left(L_{t} \mid \Delta \mathcal{F}_{t_{m}}^{a}\right), \quad t \geq 0,
$$

where $\Delta \mathcal{F}_{t_{m}}^{a}:=\sigma\left(\Delta M_{t_{m}}\right)$. Then $L^{k}$ is an $\mathbb{F}^{a}$-martingale which has jumps of the size $\left(\left\langle\Delta M_{t_{m}}, x^{*}\right\rangle\right)_{n=1}^{m}$ at $\left(t_{m}\right)_{m=1}^{k}$, and $L^{k}$ converges to $L$ in $L^{1}(\Omega)$ by $[46$, 
Theorem 3.3.2], the definition of $L$ and $M^{a}$, and by the fact that $\mathcal{F}^{a}=\otimes_{n} \Delta \mathcal{F}_{t_{n}}^{a}$, so

$$
L_{t}-L_{t}^{k}=\mathbb{E}\left(L_{t} \mid \otimes_{m>k} \Delta \mathcal{F}_{t_{m}}^{a}\right), \quad t \geq 0 .
$$

Thus $L$ is purely discontinuous with jumps of size $\left(\left\langle\Delta M_{t_{m}}, x^{*}\right\rangle\right)_{m \geq 1}$ at $\left(t_{m}\right)_{m \geq 1}$ by the fact that purely discontinuous martingales with accessible jumps form a closed subspace of $L^{1}(\Omega)$, see e.g. [116, Proposition 3.30] or [56], and hence $M^{a}$ is purely discontinuous with jumps of the size $\left(\Delta M_{t_{m}}\right)_{m \geq 1}$ at $\left(t_{m}\right)_{m \geq 1}$, so it has accessible jumps.

Step 2. Part 2. Construction of $M^{c}$. Let us now construct $M^{c}$. By the Pettis measurability theorem [46, Theorem 1.1.20] $X$ can be presumed separable. Let $\left(x_{n}\right)_{n \geq 1}$ be a dense sequence in $X$. Let $\left(x_{n}^{*}\right)_{n \geq 1}$ be a norming sequence in $X^{*}$, i.e. $\left\|x_{n}^{*}\right\|=1$ and $\left\langle x_{n}^{*}, x_{n}\right\rangle=\left\|x_{n}\right\|$ for any $n \geq 1$. For each $n \geq 1$ let $M^{n}:=\left\langle M, x_{n}^{*}\right\rangle$. Let $M^{n}=M^{n, c}+M^{n, q}+M^{n, a}$ be the corresponding canonical decomposition. By a stopping time argument, by a rescaling argument, and by Lemma 9.3 we may assume that $\mathbb{E} \sup _{t \geq 0}\left\|M_{t}\right\| \leq 1$. Then by (2.9), [56, Theorem 26.12 and 26.14] we have that

$$
\begin{aligned}
\mathbb{E} \sum_{n=1}^{\infty} \frac{1}{n^{2}}\left[M^{n, c}\right]_{\infty}^{1 / 2} & \leq \mathbb{E} \sum_{n=1}^{\infty} \frac{1}{n^{2}}\left[M^{n}\right]_{\infty}^{1 / 2} \approx \sum_{n=1}^{\infty} \frac{1}{n^{2}} \mathbb{E} \sup _{t \geq 0}\left|M_{t}^{n}\right| \\
& \leq \sum_{n=1}^{\infty} \frac{1}{n^{2}} \mathbb{E} \sup _{t \geq 0}\left|\left\langle M_{t}, x_{n}^{*}\right\rangle\right| \leq \sum_{n=1}^{\infty} \frac{1}{n^{2}} \mathbb{E} \sup _{t \geq 0}\left\|M_{t}\right\| \leq \frac{\pi^{2}}{6}
\end{aligned}
$$

so $\left(\left[M^{n, c}\right]_{\infty} / n^{4}\right)_{n \geq 1}$ are uniformly bounded a.s. Note that $M$ has independent increments, so by Theorem 9.1 it has deterministic weak local characteristics, and thus $t \mapsto\left[M^{n, c}\right]_{t} / n^{4}$ equals a finite deterministic constant a.s. for any $t \geq 0$ and $n \geq 1$. Therefore without loss of generality we may assume that all the processes $t \mapsto\left[M^{n, c}\right]_{t} / n^{4}, t \geq 0$, are continuous (see [56, Theorem 26.14]), deterministic, and uniformly bounded by (9.6). Let us then define a deterministic function

$$
A_{t}:=\sum_{n=1}^{\infty} \frac{1}{n^{6}}\left[M^{n, c}\right]_{t}+t, \quad ; t \geq 0,
$$

and let $\left(\tau_{s}^{c}\right)_{s \geq 0}$ be a deterministic time change defined by $\tau_{s}^{c}:=\inf \left\{t \geq 0: A_{t}=\right.$ $s\}$ for all $s \geq 0$. Then by Lemma 3.19 there exist a Hilbert space $H$, an enlarged probability space $(\bar{\Omega}, \overline{\mathcal{F}}, \overline{\mathbb{P}})$ with a cylindrical Brownian motion $W_{H}$ living on this space (here we set the enlargement filtration to be $\overline{\mathbb{F}}=\left(\overline{\mathcal{F}}_{t}\right)_{t \geq 0}$ is defined by $\left.\overline{\mathcal{F}}_{t}:=\sigma\left(\mathcal{F}_{t},\left.W_{H}\right|_{\left[0, A_{t}\right]}\right)\right)$, and a set of deterministic functions $f_{n}: \mathbb{R}_{+} \rightarrow H$ (note that by Lemma $3.19\left(f_{n}\right)_{n \geq 1}$ depends on $\left(\left[M^{n, c}, M^{m, c}\right]\right)_{m, n \geq 1}$ which are deterministic as $M$ has deterministic weak local characteristics by Theorem 9.1) such that $M^{n, c} \circ \tau^{c}:=f_{n} \cdot W_{H}$. Let $M_{t}^{c}:=\mathbb{E}\left(M_{t} \mid \sigma\left(W_{H}\right)\right)$ for every $t \geq 0$. Let us show that $M^{c}$ is a continuous martingale. First $M^{c}$ is a martingale as we have that for any $t \geq s \geq 0$ the martingale difference $M_{t}-M_{s}$ is independent of $\sigma\left(\left.W_{H}\right|_{\left[0, A_{s}\right]}\right)$ as $M$ is a martingale with independent increments and by the construction of $W_{H}$, so as $\sigma\left(\left.W_{H}\right|_{\left[A_{s}, \infty\right)}\right)$ is independent of $\overline{\mathcal{F}}_{s}$ we have that (here 
we for simplicity write $\sigma\left(\left.W_{H}\right|_{\left[A_{s}, \infty\right)}\right)$ instead of $\sigma\left(\left.\left(W_{H}-W_{H}\left(A_{s}\right)\right)\right|_{\left[A_{s}, \infty\right)}\right)=$ $\left.\sigma\left(\left.\mathrm{d} W_{H}\right|_{\left[A_{s}, \infty\right)}\right)\right)$

$$
\begin{aligned}
\mathbb{E}\left(M_{t}^{c}-M_{s}^{c} \mid \overline{\mathcal{F}}_{s}\right)=\mathbb{E}\left(\mathbb{E}\left(M_{t}-M_{s} \mid \sigma\left(W_{H}\right)\right) \mid \overline{\mathcal{F}}_{s}\right) & \\
& =\mathbb{E}\left(\mathbb{E}\left(M_{t}-M_{s} \mid \sigma\left(\left.W_{H}\right|_{\left[A_{s}, \infty\right)}\right)\right) \mid \overline{\mathcal{F}}_{s}\right)=0,
\end{aligned}
$$

hence $M^{c}$ is a martingale. Let us show that it is continuous. As $\left(x_{n}^{*}\right)_{n \geq 1}$ is a norming sequence, by Lemma 9.4 it is sufficient to show that $\left\langle M^{c}, x_{n}^{*}\right\rangle$ is continuous for any $n \geq 1$, so it is enough to prove that $\left\langle M^{c}, x_{n}^{*}\right\rangle=M^{n, c}$. First notice that by the construction of $W_{H}$ in Lemma 3.19 the latter depends only on $\left(M^{n, c}\right)_{n \geq 1}$. Next note that the families $\left(M^{n, q}\right)_{n \geq 1}$ and $\left(M^{n, a}\right)_{n \geq 1}$ are independent of $\left(M^{n, c}\right)_{n \geq 1}$ which follows from Step 1 of the present proof (Step 1 proves the independence directly for $\left(M^{n, q}\right)_{n=1}^{N},\left(M^{n, a}\right)_{n=1}^{N}$, and $\left(M^{n, c}\right)_{n=1}^{N}$ for any $N \geq 1$, and the desired independence follows by letting $N \rightarrow \infty$ ). Finally, we have that for any $n \geq 1$ and for any $t \geq 0$ a.s.

$$
\begin{aligned}
\left\langle M_{t}^{c}, x_{n}^{*}\right\rangle=\left\langle\mathbb{E}\left(M_{t} \mid \sigma\left(W_{H}\right)\right), x_{n}^{*}\right\rangle & =\mathbb{E}\left(\left\langle M_{t}, x_{n}^{*}\right\rangle \mid \sigma\left(W_{H}\right)\right) \\
& =\mathbb{E}\left(M^{n, c}+M^{n, q}+M^{n, a} \mid \sigma\left(W_{H}\right)\right) \stackrel{(*)}{=} M^{n, c},
\end{aligned}
$$

where $(*)$ follows from the fact that $W_{H}$ may be assumed to depend only on $\left(M^{n, c}\right)_{n \geq 1}$ and the fact that $M^{n, q}$ and $M^{n, a}$ are independent of $\left(M^{n, c}\right)_{n \geq 1}$.

Now let us show that there exists $\Phi \in \gamma\left(L^{2}\left(\mathbb{R}_{+} ; H\right), X\right)$ such that $M^{c} \circ$ $\tau^{c}=\Phi \cdot W_{H}$. First notice that for any $x^{*} \in X^{*}$ a martingale $\left\langle M^{c}, x^{*}\right\rangle \circ \tau^{c}$ is adapted with respect to the filtration $\mathbb{G}:=\left(\mathcal{G}_{s}\right)_{s \geq 1}$ generated by $W_{H}$. Therefore by the martingale representation theorem (see [97, $\S \mathrm{V} .3]$ for the case of finite dimensional $H$, the infinite dimensional case can be shown analogously) there exists a $\mathbb{G}$-predictable process $f^{x^{*}}: \mathbb{R}_{+} \times \Omega \rightarrow H$ such that $\left\langle M^{c}, x^{*}\right\rangle \circ \tau^{c}=$ $f^{x^{*}} \cdot W_{H}$. Note that $f^{x^{*}}$ is deterministic. Indeed, as $X$ can be assumed separable, the unit ball of $X^{*}$ is sequentially weak* compact by sequential Banach-Alaoglu theorem, so we may assume that $\left(x_{n}^{*}\right)_{n \geq 1}$ is weak ${ }^{*}$ dense in the unit sphere of $X^{*}$. So for a sequence $\left(y_{m}\right)_{m \geq 1} \subset\left(x_{n}^{*}\right)_{n \geq 1}$ weak $^{*}$ converging to $x^{*}$ we have that by Burkholder-Davis-Gundy inequalities [56, Theorem 26.12], by Lemma 9.3, and by the dominated convergence theorem

$$
\begin{aligned}
\mathbb{E}\left(\int_{0}^{A_{t}}\left\|f^{x^{*}}(s)-f^{y_{m}}(s)\right\|^{2} \mathrm{~d} s\right)^{1 / 2} & \approx \mathbb{E} \sup _{0 \leq t \leq T}\left|\left\langle M_{t}, x^{*}-y_{m}\right\rangle\right| \\
& \approx \mathbb{E}\left|\left\langle M_{T}, x^{*}-y_{m}\right\rangle\right| \rightarrow 0, \quad m \rightarrow \infty,
\end{aligned}
$$

so $f^{x^{*}}$ is deterministic as the limit of $f^{y_{m}}$ which are deterministic. Also note that by our assumption from the very beginning of the proof $\mathbb{E}\left\|M_{\infty}\right\|=\mathbb{E}\left\|M_{T}\right\|<\infty$ for some fixed $T>0$. Therefore as we have that $\left\langle M_{\infty}^{c}, x^{*}\right\rangle=\int_{0}^{A_{T}} f^{x^{*}} \mathrm{~d} W_{H}$ is a Gaussian random variable for any $x^{*} \in X^{*}$ (since $f^{x^{*}}$ is deterministic), $M_{\infty}^{c}$ is a Gaussian random variable itself, so by Fernique's inequality [8, Theorem 2.8.5] we have that $\mathbb{E}\left\|M_{T}^{c}\right\|^{2}<\infty$. Let $\Phi \in \gamma\left(L^{2}\left(\mathbb{R}_{+} ; H\right), X\right)$ be defined in the following way: $\Phi f=\mathbb{E} M_{\infty}^{c} N_{\infty}$, where $N=f \cdot W_{H}$ for any step deterministic 
$f \in L^{2}\left(\mathbb{R}_{+} ; H\right)$. This $\Phi$ is bounded as by Itô's isometry [27, Proposition 4.13] (see e.g. [87, Lemma 3.1.5] for the finite dimensional version) and by Hölder's inequality for any $f \in L^{2}\left(\mathbb{R}_{+} ; H\right)$ step deterministic

$$
\|\Phi f\|=\left\|\mathbb{E} M_{\infty}^{c} N_{\infty}\right\| \leq\left(\mathbb{E}\left\|M_{T}^{c}\right\|^{2}\right)^{1 / 2}\left(\mathbb{E}\left|N_{\infty}\right|^{2}\right)^{1 / 2}=\left(\mathbb{E}\left\|M_{T}^{c}\right\|^{2}\right)^{1 / 2}\|f\|_{L^{2}\left(\mathbb{R}_{+} ; X\right)},
$$

and $\gamma$-radonifying by [119, Subsection 3.2] since

$$
\mathbb{E}\left\langle M_{T}^{c}, x^{*}\right\rangle\left\langle M_{T}^{c}, y^{*}\right\rangle=\int_{0}^{\infty}\left\langle f^{x^{*}}(s), f^{y^{*}}(s)\right\rangle \mathrm{d} s \stackrel{(*)}{=}\left\langle\Phi^{*} x^{*}, \Phi^{*} y^{*}\right\rangle, \quad x^{*}, y^{*} \in X^{*},
$$

is a covariation bilinear form of a Gaussian random variable $M_{T}^{c}$, where $(*)$ follows from the fact that by Itô's isometry [27, Proposition 4.13] and by the definition of $f^{x^{*}}$

$$
\begin{aligned}
\left\langle\Phi^{*} x^{*}, f\right\rangle=\left\langle x^{*}, \Phi f\right\rangle & =\left\langle x^{*}, \mathbb{E} M_{t}^{c} \int_{0}^{\infty} f \mathrm{~d} W_{H}\right\rangle=\mathbb{E}\left\langle x^{*}, M_{t}^{c}\right\rangle \int_{0}^{\infty} f \mathrm{~d} W_{H} \\
& =\mathbb{E} \int_{0}^{\infty} f^{x^{*}} \mathrm{~d} W_{H} \int_{0}^{\infty} f \mathrm{~d} W_{H} \\
& =\int_{0}^{\infty}\left\langle f^{x^{*}}, f\right\rangle \mathrm{d} s, \quad f \in L^{2}\left(\mathbb{R}_{+} ; H\right), \quad x^{*} \in X^{*} .
\end{aligned}
$$

Now in order to show that $\Phi \cdot W_{H}$ coincides with $M^{c} \circ \tau^{c}$ it is sufficient to notice that by $(9.7) \Phi^{*} x^{*}=f^{x^{*}}$, so

$$
\left(\Phi^{*} x^{*}\right) \cdot W_{H}=f^{x^{*}} \cdot W_{H}=\left\langle M^{c}, x^{*}\right\rangle \circ \tau^{c}=\left\langle M^{c} \circ \tau^{c}, x^{*}\right\rangle, \quad x^{*} \in X^{*},
$$

and thus the desired follows from [83, Theorem 6.1].

Step 2. Part 3. Construction of $M^{q}$. Now let us show that $M^{q}:=M-$ $M^{c}-M^{a}$ is quasi-left continuous purely discontinuous and has the following form $M^{q}=\int x \mathrm{~d} \bar{\mu}^{M^{q}}=\int x \mathrm{~d} \widetilde{N}_{\nu_{n a}}$ for some Poisson random measure $N_{\nu_{n a}}$ with a compensator $\nu_{n a}$. First notice that $M^{q}$ is purely discontinuous quasi-left continuous by Corollary 9.5 as we have that for $\left(x_{n}^{*}\right)_{n \geq 1}$ exploited in Step 2 . Part $2\left\langle M^{c}, x_{n}^{*}\right\rangle$ is the continuous part of $\left\langle M, x_{n}^{*}\right\rangle$ for any $n \geq 1$. Moreover, $\left\langle M^{a}, x_{n}^{*}\right\rangle$ is the purely discontinuous with accessible jumps part of $\left\langle M, x_{n}^{*}\right\rangle$ as $M^{a}$ collects all the deterministic-time jumps of $M$, and since by Theorem 9.1 $\nu^{\left\langle M, x_{n}^{*}\right\rangle}$ is deterministic for any $n \geq 1$, its atomic part $\nu_{a}^{\left\langle M, x_{n}^{*}\right\rangle}$ (which coincides with $\nu^{\left\langle M, x_{n}^{*}\right\rangle^{a}}$ by Proposition 3.14 and Remark 3.16) has a deterministic support, which is a subset of $\left(t_{m}\right)_{m \geq 1}$ presented in Step 2. Part 1 as if $\mathbb{P}\left(\Delta\left\langle M, x_{n}^{*}\right\rangle_{t} \neq\right.$ $0)>0$ for some $t \geq 0$, then $\mathbb{P}\left(\Delta M_{t} \neq 0\right)>0$, so the jump times of $\left\langle M, x_{n}^{*}\right\rangle^{a}$ are covered by and coincide with the jump times of $\left\langle M^{a}, x_{n}^{*}\right\rangle$, consequently $\left\langle M^{a}, x_{n}^{*}\right\rangle$ is the purely discontinuous with accessible jumps part of $\left\langle M, x_{n}^{*}\right\rangle$ for any $n \geq 1$, and thus $M^{q}$ is the purely discontinuous quasi-left continuous part of the canonical decomposition of $M$.

Next let us show that $\mu^{M^{q}}$ is a Poisson random measure with a compensator $\nu^{M^{q}}=\nu_{n a}$ (the letter equality follows from Proposition 3.14, Lemma 3.15, and Remark 3.16). First note that $M^{q}$ is independent of $M^{c}$ and $M^{a}$ and 
that $M^{q}$ has independent increments. This follows from a standard finite dimensional argument (see the proof of Theorem 7.3), Step 1, and the CramérWold theorem (see [7, Theorem 29.4]). Now let us fix disjoint cylindrical sets $B_{1}, \ldots, B_{K} \in \mathcal{B}(X)$ (see the proof of Theorem 7.3) satisfying $\operatorname{dist}\left(B_{k},\{0\}\right)>\varepsilon$ for any $k=1, \ldots, K$ for some fixed $\varepsilon>0$. Then for any stopping time $\tau$ we have that

$$
\mathbb{E} \int_{[0, \tau] \times B_{k}} 1 \mathrm{~d} \nu^{M^{q}}=\mathbb{E} \int_{[0, \tau] \times B_{k}} 1 \mathrm{~d} \mu^{M^{q}}=\mathbb{E} \sum_{0 \leq s \leq \tau} \mathbf{1}_{B_{k}}\left(\Delta M_{s}^{q}\right),
$$

and the latter is locally finite if one chooses $\tau$ to be the time of $n$th jump of $M^{q}$ of value more than $\varepsilon$. Therefore we can define point processes $L^{1}, \ldots, L^{K}$ : $\mathbb{R}_{+} \times \Omega \rightarrow \mathbb{N}_{0}$ satisfying $L_{t}^{k}=\mu^{M^{q}}\left([0, t] \times B_{k}\right)$ for any $k=1, \ldots, K$ for any $t \geq 0$. But then by [56, Corollary 25.26] and Step 1 these processes are times-changed Poissons, where the time-changes are deterministic as processes $\nu_{n a}\left([0, t] \times B_{k}\right)$ are deterministic since $\nu_{n a}$ is so. Therefore $\left.\mu^{M^{q}}\right|_{\mathbb{R}_{+} \times X \backslash B(0, \varepsilon)}$ is a Poisson random measure with the compensator $\left.\nu_{n a}\right|_{\mathbb{R}_{+} \times X \backslash B(0, \varepsilon)}$ (here $B(0, \varepsilon) \subset$ $X$ is the ball in $X$ with the radius $\varepsilon$ and the centre in 0 ), and then $\mu^{M^{q}}$ is Poisson as we can send $\varepsilon \rightarrow 0$ and use the fact that by (2.14) we have that $\mu^{M^{q}}\left(\mathbb{R}_{+} \times\{0\}\right)=0$ a.s. Therefore we can set $N_{\nu_{n a}}:=\mu^{M^{q}}$ and $\widetilde{N}_{\nu_{n a}}=$ $\bar{\mu}^{M^{q}}$.

Finally, let us prove that $M^{q}=\int x \mathrm{~d} \bar{\mu}^{M^{q}}=\int x \mathrm{~d} \widetilde{N}_{\nu_{n a}}$. Recall that the definition of such an integrability was discussed in Subsection 2.9. Let us show that there exist an increasing family $\left(A_{n}\right)_{n \geq 1}$ of elements of $\mathcal{B}\left(\mathbb{R}_{+}\right) \otimes \mathcal{B}(X)$ such that $\cup_{n} A_{n}=\mathbb{R}_{+} \times X, \int_{A_{n}}\|x\| \mathrm{d} \nu_{n a}<\infty$ for any $n \geq 1$, and $\int_{A_{n}} x \mathrm{~d} \widetilde{N}_{\nu_{n a}}$ converges in $L^{1}(\Omega)$ to $M_{\infty}^{q}=M_{T}^{q}$. For every $k \in \mathbb{Z}$ let $B_{k}:=B\left(0,2^{k}\right) \backslash B\left(0,2^{k-1}\right)$. By $(9.8)$ and the discussion thereafter we have that

$$
t \mapsto \int_{[0, t] \times B_{k}}\|x\| \mathrm{d} \nu_{n a} \leq 2^{k} \int_{[0, t] \times B_{k}} 1 \mathrm{~d} \nu_{n a}<\infty, \quad t \geq 0 .
$$

Moreover, the process (9.9) is continuous as $\nu_{n a}$ is nonatomic in time. Thus for any $n \geq 1$ there exists $t_{n}^{k}$ such that $\int_{\left[0, t_{n}^{k}\right] \times B_{k}}\|x\| \mathrm{d} \nu_{n a} \leq n 2^{-k}$. Without loss of generality we may assume that $\left(t_{n}^{k}\right)_{n \geq 1}$ is an increasing sequence. Moreover, we may assume that $t_{n}^{k} \rightarrow \infty$ as $n \rightarrow \infty$ for any $k \geq 1$. For each $n \geq 1$ let us set

$$
A_{n}:=\left(\mathbb{R}_{+} \times\{0\}\right) \cup_{k \geq 1}\left(\left[0, t_{n}^{k}\right] \times B_{k}\right) .
$$

Then by the construction of $\left(t_{n}^{k}\right)_{n, k \geq 1}$ and by the fact that $\nu_{n a}\left(\mathbb{R}_{+} \times\{0\}\right)=$ $\mu^{M^{q}}\left(\mathbb{R}_{+} \times\{0\}\right)=0$ a.s. by $(2.14)$ we have that $\int_{A_{n}}\|x\| \mathrm{d} \nu_{n a}<\infty$ by (9.9) and (9.10). Let $\xi_{n}:=\int_{A_{n}} x \mathrm{~d} \widetilde{N}_{\nu_{n a}}$ for every $n \geq 1$ (see Remark 2.25). Let $\xi:=M_{\infty}^{q}$. By [46, Theorem 3.3.2] in order to show that $\xi_{n} \rightarrow \xi$ in $L^{1}(\Omega ; X)$ it is sufficient to prove that

$$
\xi_{n}=\mathbb{E}\left(\xi \mid \sigma\left(\left.N_{\nu_{n a}}\right|_{A_{n}}\right)\right), \quad n \geq 1 .
$$


Fix $n \geq 1$. To this end it is enough to show that $\left\langle\xi_{n}, x^{*}\right\rangle=\mathbb{E}\left(\left\langle\xi, x^{*}\right\rangle \mid \sigma\left(\left.N_{\nu_{n a}}\right|_{A_{n}}\right)\right)$ for any $x^{*} \in X^{*}$. Fix $x^{*} \in X^{*}$. Then $\left\langle\xi, x^{*}\right\rangle=\int_{\mathbb{R}_{+} \times X}\left\langle x, x^{*}\right\rangle \mathrm{d} \widetilde{N}_{\nu_{n a}}(\cdot, x)$ as by Burkholder-Davis-Gundy inequalities [56, Theorem 26.12] and by the dominated convergence theorem

$$
\begin{aligned}
\mathbb{E} \sup _{t \geq 0}\left|\left\langle M_{t}^{q}, x^{*}\right\rangle-\int_{[0, t] \times X \cap A_{n}}\left\langle x, x^{*}\right\rangle \mathrm{d} \widetilde{N}_{\nu_{n a}}(\cdot, x)\right| \\
\quad \approx \mathbb{E}\left(\sum_{t \geq 0} \mathbf{1}_{\bar{A}_{n}}\left(t, \Delta M^{q}\right)\left|\left\langle\Delta M^{q}, x^{*}\right\rangle\right|^{2}\right)^{1 / 2} \rightarrow 0 \quad n \rightarrow \infty,
\end{aligned}
$$

where $\bar{A}_{n} \subset \mathbb{R}_{+} \times X$ is the completion of $A_{n}$. Therefore

$$
\begin{aligned}
\mathbb{E}\left(\left\langle\xi, x^{*}\right\rangle \mid \sigma\left(N_{\nu_{n a}} \mid A_{n}\right)\right) & =\mathbb{E}\left(\int_{\mathbb{R}_{+} \times X}\left\langle x, x^{*}\right\rangle \mathrm{d} \widetilde{N}_{\nu_{n a}}(\cdot, x) \mid \sigma\left(N_{\nu_{n a}} \mid A_{n}\right)\right) \\
& =\mathbb{E}\left(\int_{A_{n}}+\int_{\bar{A}_{n}}\left\langle x, x^{*}\right\rangle \mathrm{d} \widetilde{N}_{\nu_{n a}}(\cdot, x) \mid \sigma\left(N_{\nu_{n a}} \mid A_{n}\right)\right) \\
& \stackrel{(*)}{=} \int_{A_{n}}\left\langle x, x^{*}\right\rangle \mathrm{d} \widetilde{N}_{\nu_{n a}}(\cdot, x)=\left\langle\xi_{n}, x^{*}\right\rangle,
\end{aligned}
$$

where $(*)$ holds from the fact that $N$ is a Poisson random measure so $\left.N_{\nu_{n a}}\right|_{A_{n}}$ and $\left.N_{\nu_{n a}}\right|_{\bar{A}_{n}}$ are independent, and the fact that $\mathbb{E} \int_{\bar{A}_{n}}\left\langle x, x^{*}\right\rangle \mathrm{d} \widetilde{N}_{\nu_{n a}}(\cdot, x)=0$. Therefore (9.11) holds true, and thus $\xi_{n} \rightarrow \xi$ in $L^{1}(\Omega ; X)$ by the Itô-Nisio theorem [47, Theorem 6.4.1], so $M^{q}=\int x \mathrm{~d} \widetilde{N}_{\nu_{n a}}(\cdot, x)$.

Step 3. Proving (9.1). Finally let us show (9.1). This estimates follow analogously finite dimensional case proven in Step 1, with exploiting the fact that $M^{c}$, $M^{q}$, and $M^{a}$ are independent by the Cramér-Wold theorem (see [7, Theorem 29.4]) and by Step 1.

Remark 9.6. Note that $\Phi$ is locally in $\gamma\left(L^{2}\left(\mathbb{R}_{+} ; H\right), X\right)$, so by [119, Subsection 3.2] and by (9.12) we have that $\gamma\left(\llbracket M^{c} \rrbracket_{t}\right)=\|\Phi\|_{\gamma\left(L^{2}([0, t] ; H), X\right)}<\infty$.

The following corollary is an extension of the famous result of Grigelionis [42] (see also [52, p. 106]) to infinite dimensions.

Corollary 9.7. Let $X$ be a Banach space, $M: \mathbb{R}_{+} \times \Omega \rightarrow X$ be a martingale. Then $M$ has independent increments of and only if it has local characteristics which are deterministic.

Proof. The "if" part follows from Theorem 9.1. Let us show the "only if" part. The fact that $M$ admits the Meyer-Yoeurp decomposition and that $\nu^{M}$ is deterministic was shown in Theorem 9.2. Let us show that $\llbracket M^{c} \rrbracket$ exists. This follows from the fact that for any $t \geq 0$ a.s. for any $x^{*}, y^{*} \in X^{*}$ by the Kazamaki theorem [56, Theorem 17.24] and by (2.17) (recall that $\Phi^{*} x^{*}$ is locally in $L^{2}\left(\mathbb{R}_{+} ; H\right)$ 
for any $\left.x^{*} \in X^{*}\right)$

$$
\begin{aligned}
\left|\llbracket M^{c} \rrbracket_{t}\left(x^{*}, y^{*}\right)\right| & =\left|\left[\left\langle M^{c}, x^{*}\right\rangle,\left\langle M^{c}, y^{*}\right\rangle\right]_{t}\right| \\
& =\left|\left[\int \Phi^{*} x^{*} \mathrm{~d} W_{H}, \int \Phi^{*} y^{*} \mathrm{~d} W_{H}\right]_{A_{t}}\right| \\
& =\left|\int_{0}^{A_{t}}\left\langle\Phi x^{*}(s), \Phi^{*} y^{*}(s)\right\rangle \mathrm{d} s\right| \\
& \leq\|\Phi\|_{\mathcal{L}\left(L^{2}\left(\left[0, A_{t}\right]\right), X\right)}^{2}\left\|x^{*}\right\|\left\|y^{*}\right\|,
\end{aligned}
$$

so $\llbracket M^{c} \rrbracket_{t}$ is a.s. a bounded bilinear form.

Corollary 9.8. Let $X$ be a Banach space, $M: \mathbb{R}_{+} \times \Omega \rightarrow X$ be a martingale with independent increments satisfying $M_{0}=0$. Then the distribution of $M$ is uniquely determined by its local characteristics.

Proof. By Theorem 9.2 it is sufficient to show that each part of the canonical decomposition $M=M^{c}+M^{q}+M^{a}$ is uniquely determined by its characteristics. To this end it is enough to notice that the distribution of $M^{c}$ depends only on $\Phi$ and $\left(A_{t}\right)_{t \geq 0}$ which depends only on $\llbracket M^{c} \rrbracket$, the distribution of $M^{q}$ depends only on $\nu_{n a}$, and the distribution of $M^{a}$ depends only on $\nu_{a}$.

\section{The approach of Jacod, Kwapień, and Woyczyński}

In the present section we discover the infinite dimensional analogue of the celebrated result of Jacod [50] and Kwapień and Woyczyński [64], which says that if one discretize a real-valued quasi-left continuous martingale $M$ by creating a sequence $d^{n}=\left(d_{k}^{n}\right)_{k=1}^{n}=\left(M_{T k / n}-M_{T(k-1) / n}\right)_{k=1}^{n}$, and if one considers a decoupled tangent martingale difference sequence $\tilde{d}^{n}=\left(\tilde{d}_{k}^{n}\right)_{k=1}^{n}$ to $d^{n}$, then $\tilde{d}^{n}$ converges in distribution to a decoupled tangent martingale $\widetilde{M}$. The goal of the present section is to extend this statement to UMD-valued general local martingales.

Before stating the main theorem of the section we will need the following definitions. First recall that $\mathcal{D}([0, T], X)$ denotes the Skorokhod space of all $X$ valued càdlàg functions on $[0, T]$ (see Definition 2.2). Throughout this section we will assume the Skorokhod space to be endowed with the Skorokhod topology (instead of the sup-norm topology, see Remark 10.9) which is generated by the Skorokhod metric which has the following form. Let $F, G \in \mathcal{D}([0, T], X)$. Then

$$
d_{J_{1}}(F, G):=\inf _{\lambda}\left(\sup _{0 \leq t \leq T}|\lambda(t)-t|+\sup _{0 \leq t \leq T}\|F(t)-G(\lambda(t))\|\right),
$$

where the infimum is taken over all nondecreasing functions $\lambda:[0, T] \rightarrow[0, T]$. Note that

$$
d_{J_{1}}(F, G) \leq\|F-G\|_{\infty} .
$$

We refer the reader to $[6,7,53,104,112]$ for further information on Skorokhod spaces. 
Definition 10.1. Let $(\mathcal{D}, d)$ be a metric space. A sequence of $\mathcal{D}$-valued random variables $\left(\xi_{n}\right)_{n \geq 1}$ converges to an $\mathcal{D}$-valued random variable $\xi$ in distribution if the distributions of $\left(\xi_{n}\right)_{n \geq 1}$ converge weakly to the distribution of $\xi$, i.e. $\mathbb{E} f\left(\xi_{n}\right) \rightarrow \mathbb{E} f(\xi)$ as $n \rightarrow \infty$ for any bounded continuous function $f: \mathcal{D} \rightarrow \mathbb{R}$.

We refer the reader to $[9,103]$ for further details on convergence in distribution and on weak convergence.

Remark 10.2. Assume that $\mathcal{D}$ in Definition 10.1 is a locally convex space. Without loss of generality by [9, Remark 8.3.1 and the proof of Theorem 8.2.3] we may assume that $f$ in the definition above is Lipschitz. Moreover, by multiplying $f$ by a constant we may assume that $\|f\|_{\infty} \leq 1$ and that

$$
|f(x)-f(y)| \leq d(x, y), \quad x, y \in X .
$$

Let $X$ be a Banach space, $M: \mathbb{R}_{+} \times \Omega \rightarrow X$ be a local martingale. Fix $T>0$ and for each $n \geq 1$ define

$$
d_{k}^{n}:=M_{T k / n}-M_{T(k-1) / n}, \quad k=1, \ldots, n .
$$

For each $n \geq 1$, let $\left(\tilde{d}_{k}^{n}\right)_{k=1}^{n}$ be a decoupled tangent sequence. Let

$$
\widetilde{M}_{t}^{n}:=\sum_{k: T k / n \leq t} \tilde{d}_{k}^{n}, \quad t \geq 0 .
$$

First start with a classical real-valued result. The following theorem was shown by Jacod in [49, 50] (see also Kwapień and Woyczyński [64, p. 176], and [52, Chapter VI-VIII]).

Theorem 10.3. Let $X$ be a finite dimensional Banach space, $M: \mathbb{R}_{+} \times \Omega \rightarrow X$ be a quasi-left continuous local martingale that starts at zero, $T>0,\left(\widetilde{M}^{n}\right)_{n \geq 1}$ be defined by (10.3). Then $\left(\widetilde{M}^{n}\right)_{n \geq 1}$ converges in distribution as random variables with values in $\mathcal{D}([0, T], X)$ to a decoupled tangent process $\widetilde{M}$ of $M$.

The goal of the present subsection is to extend Theorem 10.3 to infinite dimensions and to general local martingales.

Theorem 10.4. Let $X$ be a UMD Banach space, $M: \mathbb{R}_{+} \times \Omega \rightarrow X$ be a local martingale that starts at zero, $T>0,\left(\widetilde{M}^{n}\right)_{n \geq 1}$ be defined by (10.3). Then $\left(\widetilde{M}^{n}\right)_{n \geq 1}$ converges in distribution as random variables with values in $\mathcal{D}([0, T], X)$ to a decoupled tangent process $\widetilde{M}$ of $M$.

In order to prove Theorem 10.4 we will need several intermediate steps outlined here as lemmas.

Lemma 10.5. Let $X$ be a UMD Banach space, $M^{1}, \ldots, M^{n}: \mathbb{R}_{+} \times \Omega \rightarrow$ $X$ be local martingales. Then there exist corresponding decoupled tangent local martingales $\widetilde{M}^{1}, \ldots, \widetilde{M}^{n}$ such that $\alpha^{1} \widetilde{M}^{1}+\ldots+\alpha^{n} \widetilde{M}^{n}$ is a decoupled tangent local martingale to $\alpha^{1} M^{1}+\ldots+\alpha^{n} M^{n}$ for any $\alpha^{1}, \ldots, \alpha^{n} \in \mathbb{R}$. 
Proof. First notice that the $n$th power $X \times \cdots \times X$ of $X$ (endowed with the $\ell_{n}^{p}$ product norm for any $1<p<\infty$ ) is a UMD Banach space (see e.g. [46, Proposition 4.2.17]). Then it is sufficient to consider an $X \times \cdots \times X$-valued local martingale $\left(M^{1}, \ldots, M^{n}\right)$ and set $\widetilde{M}^{1}, \ldots, \widetilde{M}^{n}$ to be such that $\left(\widetilde{M}^{1}, \ldots, \widetilde{M}^{n}\right)$ is a decoupled tangent local martingale to $\left(M^{1}, \ldots, M^{n}\right)$. Then the lemma follows from Theorem A.1 and the fact that

$$
\left(x^{1}, \ldots, x^{n}\right) \mapsto \alpha^{1} x^{1}+\ldots+\alpha^{n} x^{n}, \quad x^{1}, \ldots, x^{n} \in X,
$$

is a bounded linear operator from $X \times \cdots \times X$ to $X$.

Lemma 10.6. Let $X$ be a UMD Banach space, $M,\left(M_{m}\right)_{m \geq 1}$ be local $X$-valued martingales such that $\mathbb{E} \sup _{t \geq 0}\left\|M_{t}-\left(M_{m}\right)_{t}\right\| \rightarrow 0$ as $m \rightarrow \infty$. Let $T>0$, and let $\widetilde{M}^{n}$ and $\left(\widetilde{M}_{m}^{n}\right)_{m \geq 1}$ be defined analogously to (10.3). Assume additionally that $\widetilde{M}_{m}^{n}$ converges in distribution to a local martingale $\widetilde{M}_{m}$ which is decoupled tangent to $M_{m}$ for any $m \geq 1$. Then $\widetilde{M}^{n}$ converges in distribution to a local martingale $\widetilde{M}$ which is decoupled tangent to $M$.

Proof. Fix a continuous bounded function $f: \mathcal{D}([0, T], X) \rightarrow \mathbb{R}$. Our goal is to show that $\mathbb{E} f(\widetilde{M})=\lim _{n \rightarrow \infty} \mathbb{E} f\left(\widetilde{M}^{n}\right)$. Thanks to Remark 10.2 we may assume that $f$ is Lipschitz, $\|f\|_{\infty} \leq 1$, and that by (10.1) $|f(x)-f(y)| \leq d_{J_{1}}(x, y) \leq$ $\|x-y\|$ for any $x, y \in \mathcal{D}([0, T], X)$.

Assume the converse, i.e. that $\mathbb{E} f(\widetilde{M}) \neq \lim _{n \rightarrow \infty} \mathbb{E} f\left(\widetilde{M}^{n}\right)$. Then we can identify our sequence with a subsequence such that

$$
\mathbb{E} f(\widetilde{M}) \leq \mathbb{E} f\left(\widetilde{M}^{n}\right)-\delta
$$

for some fixed $\delta>0$ for any $n \geq 1$ (we may use $-f$ instead of $f$ if needed). Fix $\varepsilon>0$. Let $m \geq 1$ be such that $\mathbb{E} \sup _{0 \leq t \leq T}\left\|M_{t}-\left(M_{m}\right)_{t}\right\|<\varepsilon$. Then by Lemma 10.5 and by (3.3) we have that

$$
\mathbb{E} \sup _{0 \leq t \leq T}\left\|\widetilde{M}_{t}-\left(\widetilde{M}_{m}\right)_{t}\right\|<C_{X} \varepsilon
$$

for some universal constant $C_{X}>0$. For this $m$ by the assumption of the theorem we can find $n \geq 1$ such that

$$
\mathbb{E} f\left(\left(\widetilde{M}_{m}\right)_{t}\right) \geq \mathbb{E} f\left(\left(\widetilde{M}_{m}^{n}\right)_{t}\right)-\varepsilon .
$$

Finally, by the construction (10.3) of $\widetilde{M}_{m}^{n}$ and $\widetilde{M}^{n}$ and by Lemma 10.5 we may assume that $\widetilde{M}^{n}-\widetilde{M}_{m}^{n}$ is a discrete decoupled tangent martingale to $M_{t}-M_{m}$ constructed by (10.3) so analogously to (10.5) we have that

$$
\mathbb{E} \sup _{0 \leq t \leq T}\left\|\widetilde{M}_{t}^{n}-\left(\widetilde{M}_{m}^{n}\right)_{t}\right\| \leq C_{X} \mathbb{E} \sup _{0 \leq t \leq T}\left\|M_{t}-\left(M_{m}\right)_{t}\right\|<C_{X} \varepsilon
$$

Therefore summing up (10.5), (10.6), and (10.7) together with the Lipschitzivity of $f$ we have that $\mathbb{E} f(\widetilde{M}) \geq \mathbb{E} f\left(\widetilde{M}^{n}\right)-\varepsilon-2 C_{X} \varepsilon$, so (10.4) does not hold if $\varepsilon$ is small enough. 
Lemma 10.7. Let $M^{c}: \mathbb{R}_{+} \times \Omega \rightarrow \mathbb{R}$ be a continuous local martingale, let $n \geq 1, J=\{1, \ldots, n\}$, and let $\mu$ be an integer-valued optional random measure on $\mathbb{R}_{+} \times J$ with a compensator $\nu$. Assume that there exists a random timechange $\tau=\left(\tau_{t}\right)_{t \geq 0}$ such that $\left[M^{c}\right]_{\tau_{t}}=t$, and random time-changes $\left(\tau_{t}^{j}\right)_{t \geq 0}$, $j=1, \ldots, n$ such that

$$
\nu\left(\left[0, \tau_{t}^{j}\right] \times\{j\}\right)=t, \quad t \geq 0, \quad j=1, \ldots, n .
$$

Then the process $t \mapsto M_{\tau_{t}}^{c}$ and random measures $(t, j) \mapsto \tilde{\mu}^{j}([0, t] \times\{j\}):=$ $\mu\left(\left[0, \tau_{t}^{j}\right] \times\{j\}\right), j=1, \ldots, n$, are mutually independent.

Proof. The fact that $\left(\tilde{\mu}^{j}\right)_{j=1}^{n}$ are independent standard Poisson random measures follows from [56, Corollary 25.26]. Let us show that $W:=M^{c} \circ \tau$ is independent of $\left(\tilde{\mu}^{j}\right)_{j=1}^{n}$. Without loss of generality assume that $n=1$ (the proof for $n>1$ is analogous $)$. Let $\tilde{\mu}=\tilde{\mu}^{1}, \widetilde{N}_{t}=\tilde{\mu}([0, t] \times\{1\})-t, t \geq 0$. Then $W$ is a standard Brownian motion by [56, Theorem 18.3 and 18.4] and $\widetilde{N}$ is a standard compensated Poisson process by [56, Corollary 25.26], and for any $0 \leq t_{1}<\ldots<t_{N}$, $0 \leq s_{1}<\ldots<s_{N}$ and for any numbers $\alpha_{1}, \ldots, \alpha_{N}$ and $\beta_{1}, \ldots, \beta_{N}$ we have that (here we set $t_{0}=s_{0}=\alpha_{0}=\beta_{0}=0$ and $d \alpha_{k}:=\alpha_{k}-\alpha_{k-1}, d \beta_{k}:=\alpha_{k}-\beta_{k-1}$ for any $k=1, \ldots, N)$

$$
\begin{array}{r}
e^{-\frac{1}{2} \sum_{k=1}^{N}\left(\left[d \alpha_{k} M^{c}\right]_{\tau_{t_{k}}}-\left[d \alpha_{k} M^{c}\right]_{\tau_{t_{k-1}}}\right)+\sum_{k=1}^{N} \int{\left(\tau_{s_{k-1}}^{1}, \tau_{s_{k}}^{1}\right] \times\{1\}} e^{i d \beta_{k}-1-i d \beta_{k} \mathrm{~d} \nu}} \\
=e^{-\frac{1}{2} \sum_{k=1}^{N} d \alpha_{k}^{2}\left(t_{k}-t_{k-1}\right)+\sum_{k=1}^{N}\left(e^{i d \beta_{k}}-1-i d \beta_{k}\right)\left(s_{k}-s_{k-1}\right)}
\end{array}
$$

is a constant a.s., so by a stopping time argument and by [52, II.4.16] we have that

$$
\begin{aligned}
\mathbb{E} & e^{i\left(\sum_{k=1}^{N} \alpha_{k} W_{t_{k}}+\sum_{k=1}^{N} \beta_{k} \widetilde{N}_{s_{k}}\right)} \\
& =e^{-\frac{1}{2} \sum_{k=1}^{N} d \alpha_{k}^{2}\left(t_{k}-t_{k-1}\right)+\sum_{k=1}^{N}\left(e^{i d \beta_{k}}-1-i d \beta_{k}\right)\left(s_{k}-s_{k-1}\right)} \\
& =e^{-\frac{1}{2} \sum_{k=1}^{N} d \alpha_{k}^{2}\left(t_{k}-t_{k-1}\right)} e^{\sum_{k=1}^{N}\left(e^{i d \beta_{k}}-1-i d \beta_{k}\right)\left(s_{k}-s_{k-1}\right)} \\
& =\mathbb{E} e^{i \sum_{k=1}^{N} \alpha_{k} W_{t_{k}}} \mathbb{E} e^{\sum_{k=1}^{N} \beta_{k} \widetilde{N}_{s_{k}}},
\end{aligned}
$$

so $\left(W_{t_{k}}\right)_{k=1}^{N}$ and $\left(\widetilde{N}_{s_{k}}\right)_{k=1}^{N}$ are independent by [103, Theorem II.12.4] as $\alpha_{1}, \ldots, \alpha_{N}$ and $\beta_{1}, \ldots, \beta_{N}$ are arbitrary. As $t_{1}, \ldots, t_{N}$ and $s_{1}, \ldots, s_{N}$ are arbitrary, $W$ and $\widetilde{N}$ (hence, $W$ and $\tilde{\mu}$ ) are independent.

Let us finally prove Theorem 10.4.

Proof of Theorem 10.4. First, by a stopping time argument and by Theorem A.3 we may assume that $\mathbb{E} \sup _{0 \leq t \leq T}\left\|M_{t}\right\|<\infty$. By Lemma 10.6 and by a stopping time argument it is sufficient to show Theorem 10.4 for a martingale $M$ from a dense subset of martingales. In particular, by [119, Subsection 7.5] we may assume that $X$ is finite dimensional. Let $M=M^{c}+M^{q}+M^{a}$ be the canonical decomposition. We will approximate each part of the canonical decomposition separately (we are allowed to do so by (2.9)). Our goal is to exploit Lemma 10.5 
and approximate $M$ in such a way that $\widetilde{M}^{n}$ almost coincides with $\widetilde{M}$ for any $n$ big enough.

Step 1: approximation of $M^{c}$. By the proof of Theorem 3.18 we may assume that there exists an invertible time-change $\left(\tau_{s}\right)_{s \geq 0}$ with an inverse time-change $\left(A_{t}\right)_{t \geq 0}$, a separable Hilbert space $H$, an elementary $\left(\mathcal{F}_{\tau_{s}}\right)_{s \geq 0}$-predictable process $\Phi: \mathbb{R}_{+} \times \Omega \rightarrow \mathcal{L}(H, X)$, and an $\left(\mathcal{F}_{\tau_{s}}\right)_{s \geq 0}$-adapted cylindrical Brownian motion $W_{H}$ such that $M^{c} \circ \tau=\Phi \cdot W_{H}$, or, in other words, $M_{t}^{c}=\int_{0}^{A_{t}} \Phi \mathrm{d} W_{H}$. By an approximation argument and the definition of a stochastic integral (see Subsection 2.10) we may assume that $\Phi$ is elementary $\left(\mathcal{F}_{\tau_{s}}\right)_{s \geq 0}$-predictable with respect to the mesh

$$
\left\{A_{0}, A_{T / N_{0}}, \ldots, A_{T\left(N_{0}-1\right) / N_{0}}, A_{T}\right\}
$$

so $(\Phi \circ A)_{t}$ is $\left(\mathcal{F}_{T(k-1) / N_{0}}\right)_{t \geq 0}$-measurable for any $\frac{k-1}{N_{0}} T \leq t<\frac{k}{N_{0}} T$ for some fixed big natural number $N_{0}$. Moreover, for $N_{0}$ big enough we can approximate $M^{c}$ by a continuous martingale (adapted to another filtration) $M^{c, N_{0}}$ in the following way.

By a stopping time argument and by the fact that $A$ is continuous we may assume that $A_{T} \leq C$ a.s for some fixed $C>0$. Let $\bar{W}_{H}$ be a cylindrical Brownian motion such that

$\left.\bar{W}_{H}\right|_{\left[C(k-1), C(k-1)+A_{T k / N_{0}}-A_{T(k-1) / N_{0}}\right]}=\left.W_{H}\right|_{\left[A_{T(k-1) / N_{0}}, A_{T k / N_{0}}\right]}, \quad k=1, \ldots, N_{0}$.

Set $A^{\prime}: \mathbb{R}_{+} \times \Omega \rightarrow \mathbb{R}_{+}$to be

$$
A_{t}^{\prime}:= \begin{cases}C(k-1)+A_{t}-A_{T(k-1) / N_{0}}, & \frac{k-1}{N_{0}} T \leq t<\frac{k}{N_{0}} T, \quad k=1, \ldots, N_{0}, \\ C\left(N_{0}\right)+A_{T}-A_{T\left(N_{0}-1\right) / N_{0}}, & t \geq T,\end{cases}
$$

and set $\Phi^{\prime}: \mathbb{R}_{+} \times \Omega \rightarrow \mathcal{L}(H, X)$ to be such that

$$
\left.\Phi^{\prime}\right|_{\left(C(k-1), C(k-1)+A_{T k / N_{0}}-A_{T(k-1) / N_{0}}\right]}=\left.\Phi\right|_{\left(A_{T(k-1) / N_{0}}, A_{T k / N_{0}}\right]}, \quad k=1, \ldots, N_{0},
$$

and zero otherwise. (Recall that we assumed $\Phi$ to be elementary predictable with respect to the mesh (10.8), so $\Phi$ is a.s. a constant on $\left(A_{T(k-1) / N_{0}}, A_{T k / N_{0}}\right]$, and thus $\Phi^{\prime}$ is a.s. a constant on $\left.\left(C(k-1), C(k-1)+A_{T k / N_{0}}-A_{T(k-1) / N_{0}}\right]\right)$. By the definition of $\Phi^{\prime}$ and $\bar{W}_{H}$ we have that $M_{t}^{c}=\int_{0}^{A_{t}^{\prime}} \Phi^{\prime} \mathrm{d} \bar{W}_{H}$ for any $t \geq 0$. Now let us construct the desired $M^{c, N_{0}}$ in the following way. Let $\bar{A}: \mathbb{R}_{+} \times \Omega \rightarrow \mathbb{R}_{+}$ be defined by

$$
\bar{A}_{t}:= \begin{cases}C(k-1)+\mathbb{E}\left(A_{t}-A_{T(k-1) / N_{0}} \mid \mathcal{F}_{T(k-1) / N_{0}}\right), & \frac{k-1}{N_{0}} T \leq t<\frac{k}{N_{0}} T, \\ C\left(N_{0}\right)+\mathbb{E}\left(A_{T}-A_{T\left(N_{0}-1\right) / N_{0}} \mid \mathcal{F}_{T(k-1) / N_{0}}\right), & t \geq T,\end{cases}
$$

and let $\bar{\Phi}: \mathbb{R}_{+} \times \Omega \rightarrow \mathcal{L}(H, X)$ be

$\bar{\Phi}_{s}:= \begin{cases}\Phi_{C(k-1)}^{\prime}, & C(k-1) \leq s \leq C(k-1)+\mathbb{E}\left(A_{t}-A_{T(k-1) / N_{0}} \mid \mathcal{F}_{T(k-1) / N_{0}}\right), \\ 0, & \text { otherwise. }\end{cases}$ 
Let $M_{t}^{c, N_{0}}:=\int_{0}^{\bar{A}_{t}} \bar{\Phi} \mathrm{d} \bar{W}_{H}, t \geq 0$. Let us show that

$$
\mathbb{E} \sup _{k=1}^{N_{0}}\left\|M_{T k / N_{0}}^{c}-M_{T k / N_{0}}^{c, N_{0}}\right\| \rightarrow 0, \quad N_{0} \rightarrow \infty .
$$

(Though the limit here cannot be considered literally as $\Phi \circ A$ is step with respect to the mesh (10.8) for some fixed $N_{0}$, but not for any big $N_{0}$, we still can send $N_{0}$ to infinity, as for any $N \gg N_{0}$ we have that $\Phi \circ A$ is almost step with respect to the mesh $\left\{A_{0}, A_{T / N}, \ldots, A_{T(N-1) / N}, A_{T}\right\}$ as for such big $N$ by the boundedness of $\Phi$ the process $\Phi$ can simply be assumed zero on $\left(A_{T(k-1) / N}, A_{T k / N}\right]$ if one of the knots of (10.8) turned out to be in $\left(A_{T(k-1) / N}, A_{T k / N}\right]$. This does not change much the norm of the stochastic integral by [56, Theorem 26.12] and (2.17)).

Let $L_{t}^{c}:=\int_{0}^{t} \bar{\Phi} a^{c, N_{0}} \mathrm{~d} \bar{W}_{H}$, where

$$
\begin{aligned}
& a^{c, N_{0}}(t) \\
& \quad:=\sum_{k=1}^{N_{0}} \mathbf{1}_{\left[C(k-1)+\mathbb{E}\left(A_{T k / N_{0}}-A_{T(k-1) / N_{0}} \mid \mathcal{F}_{T(k-1) / N_{0}}\right), C(k-1)+A_{T k / N_{0}}-A_{T(k-1) / N_{0}}\right]}(t),
\end{aligned}
$$

for any $t \geq 0$. Then

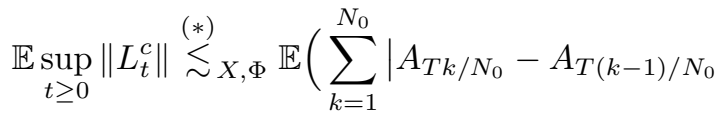

$$
\begin{aligned}
& \left.-\mathbb{E}\left(A_{T k / N_{0}}-A_{T(k-1) / N_{0}} \mid \mathcal{F}_{T(k-1) / N_{0}}\right) \mid\right)^{1 / 2} \\
& =\mathbb{E}\left(\sum_{k=1}^{N_{0}}\left|A_{T k / N_{0}}-\mathbb{E}\left(A_{T k / N_{0}} \mid \mathcal{F}_{T(k-1) / N_{0}}\right)\right|\right)^{1 / 2} \stackrel{(* *)}{\rightarrow} 0, \quad N_{0} \rightarrow \infty,
\end{aligned}
$$

where $(*)$ holds by $(2.18)$, the fact that $X$ is finite dimensional, and the fact that $\Phi$ is elementary predictable, and hence bounded, while (**) follows from Lemma 3.24. Moreover, if for any $t \geq 0$ we set

$$
\begin{aligned}
b^{c, N_{0}}(t) \\
\quad:=\sum_{k=1}^{N_{0}} \mathbf{1}_{\left[C(k-1), C(k-1)+\mathbb{E}\left(A_{T k / N_{0}}-A_{T(k-1) / N_{0}} \mid \mathcal{F}_{T(k-1) / N_{0}}\right) \wedge\left(A_{T k / N_{0}}-A_{T(k-1) / N_{0}}\right)\right]}(t),
\end{aligned}
$$

then

$$
\begin{aligned}
& \mathbb{E} \sup _{k=1}^{N_{0}}\left\|M_{T k / N_{0}}^{c}-\left(M_{T k / N_{0}}^{c, N_{0}}-L_{T K / N_{0}}^{c}\right)\right\| \leq \mathbb{E} \sup _{t \geq 0}\left\|\int_{0}^{t} \Phi^{\prime}\left(1-b^{c, N_{0}}\right) \mathrm{d} \bar{W}_{H}\right\| \\
& \stackrel{(*)}{\lesssim_{X, \Phi}} \mathbb{E}\left(\sum_{k=1}^{N_{0}} \mid A_{T k / N_{0}} \wedge \mathbb{E}\left(A_{T k / N_{0}} \mid \mathcal{F}_{T(k-1) / N_{0}}\right)\right. \\
& \left.-\mathbb{E}\left(A_{T k / N_{0}} \mid \mathcal{F}_{T(k-1) / N_{0}}\right) \mid\right)^{1 / 2}
\end{aligned}
$$




$$
\leq \mathbb{E}\left(\sum_{k=1}^{N_{0}}\left|A_{T k / N_{0}}-\mathbb{E}\left(A_{T k / N_{0}} \mid \mathcal{F}_{T(k-1) / N_{0}}\right)\right|\right)^{1 / 2} \stackrel{(* *)}{\rightarrow} 0, \quad N_{0} \rightarrow \infty,
$$

where $(*)$ follows from $(2.18)$, and $(* *)$ follows from Lemma 3.24. Thus (10.9) follows.

Step 2: approximation of $M^{q}$. By Theorem 3.30 and by Lemma 10.6 we may assume that $M^{q}=\int_{[0, \cdot] \times J} F \mathrm{~d} \bar{\mu}$ for some finite set $J=\{1, \ldots, n\}$, for some elementary predictable $F: \mathbb{R}_{+} \times \Omega \times J \rightarrow X$, and for some quasi-left continuous random measure $\mu$ on $\mathbb{R}_{+} \times J$ with a compensator $\nu$ so that $\nu([0, t] \times\{j\})<\infty$ a.s. for any $t \geq 0$ and $\nu\left(\mathbb{R}_{+} \times\{j\}\right)=\infty$ a.s. for any $j \in J$ (the latter can be done e.g. similarly to Step 4 of the proof of Theorem 3.22). By a stopping time argument and by Lemma 10.6 we may assume that $\nu([0, T] \times J)<C$ a.s. for the same constant $C$ as in Step 1 of the present proof. Analogously to Step 1 of the proof of Theorem 3.22 we may construct an approximation $M^{q, N_{0}}$ of $M^{q}$ such that $\nu^{M^{q, N_{0}}}([0, t] \times\{j\})$ is $\mathcal{F}_{T(k-1) / N_{0}}$-measurable for any $\frac{k-1}{N_{0}} T \leq t<\frac{k}{N_{0}} T$. Indeed, as it was done in the proof of Theorem 3.22, we can approximate $M^{q}$ with a process $M^{q, N_{0}}$ which is constructed analogously to (3.32) in the following way. Let $N_{0}$ be so big that $M^{q}$ has the form (which is analogous to the form (3.31))

$M_{t}^{q}=\sum_{k=1}^{N_{0}} \sum_{j=1}^{n} \bar{\eta}\left(\left[C(k-1), C(k-1)+\nu^{j}\left[\frac{T(k-1)}{N_{0}} \wedge t, \frac{T K}{N_{0}} \wedge t\right)\right) \times\{j\}\right) \xi_{k, j}, \quad t \geq 0$,

where $\eta$ is some standard Poisson random measure on $\mathbb{R}_{+} \times J$ with a compensator $\nu_{\eta}, \bar{\eta}:=\eta-\nu_{\eta}, \xi_{k, j}$ is $\mathcal{F}_{T(k-1) / N_{0}}$-measurable and simple. For any $j=1, \ldots, n$ let $\nu^{j}$ be defined by (3.26) and let

$$
\begin{aligned}
M_{t}^{q, N_{0}} & =\sum_{k=1}^{N_{0}} \sum_{j=1}^{n} \bar{\eta}([C(k-1), \\
& \left.\left.C(k-1)+\mathbb{E}\left(\nu^{j}\left[\frac{T(k-1)}{N_{0}} \wedge t, \frac{T k}{N_{0}} \wedge t\right) \mid \mathcal{F}_{T(k-1) / N_{0}}\right)\right) \times\{j\}\right) \xi_{k, j}, \quad t \geq 0 .
\end{aligned}
$$

It remains to notice that

$$
\mathbb{E} \sup _{k=1}^{N_{0}}\left\|M_{T k / N_{0}}^{q}-M_{T k / N_{0}}^{q, N_{0}}\right\| \rightarrow 0, \quad N_{0} \rightarrow \infty,
$$

which follows analogously (3.34) and (3.35). (Here we are allowed to send $N_{0}$ to infinity for the same reason as in Step 1).

Step 3: approximation of $M^{a}$. By Lemma 10.6 and by (B.4) we may assume that $M^{a}$ has its jumps in predictable stopping times $0 \leq \sigma_{1}<\ldots<\sigma_{m} \leq$ T. Moreover, by Lemma 10.6, Lemma 3.38, and the proof of Proposition 3.37 we may assume that $\Delta M_{\sigma_{1}}, \ldots, \Delta M_{\sigma_{m}}$ are simple in $L^{\infty}(\Omega ; X)$ and thus have values in a finite dimensional subspace of $X$. In addition assume that there exists $\delta>0$ such that for all differences we have that a.s.

$$
\sigma_{2}-\sigma_{1}, \ldots, \sigma_{m}-\sigma_{m-1} \geq \delta \text {. }
$$


We can assume so as we can approximate $M^{q}$ by the following martingale

$$
M_{t}^{a, \delta}:=\Delta M_{\sigma_{1}} \mathbf{1}_{[0, t]}\left(\sigma_{1}\right)+\sum_{\ell=2}^{m} \Delta M_{\sigma_{\ell}} \mathbf{1}_{[0, t]}\left(\sigma_{\ell}\right) \mathbf{1}_{\sigma_{2} \geq \sigma_{1}+\delta, \ldots, \sigma_{\ell} \geq \sigma_{\ell-1}+\delta}, \quad t \geq 0,
$$

which is a martingale by Lemma 2.11, and set $\sigma_{1}^{\prime}:=\sigma_{1}$ and

$$
\sigma_{\ell}^{\prime}:= \begin{cases}\sigma_{\ell}, & \text { if } \sigma_{2} \geq \sigma_{1}+\delta, \ldots, \sigma_{\ell} \geq \sigma_{\ell-1}+\delta \\ \sigma_{\ell-1}^{\prime}+\delta, & \text { otherwise }\end{cases}
$$

for any $\ell=2, \ldots, m$. Indeed, as $M$ takes valued in a finite dimensional subspace of $X, X$ can be assumed finite dimensional, so by the finite dimensional version of [56, Theorem 26.12]

$$
\mathbb{E} \sup _{t \geq 0}\left\|M_{t}^{a}-M_{t}^{a, \delta}\right\| \approx \mathbb{E}\left(\sum_{\ell=1}^{m}\left\|\Delta M_{\sigma_{\ell}}\right\|^{2} \mathbf{1}_{\sigma_{2} \geq \sigma_{1}+\delta, \ldots, \sigma_{\ell} \geq \sigma_{\ell-1}+\delta}\right)^{1 / 2} \rightarrow 0, \quad \delta \rightarrow 0,
$$

where the latter holds by the dominated convergence theorem and by the fact that a.s.

$$
\max \left\{\sigma_{2}-\sigma_{1}, \ldots, \sigma_{m}-\sigma_{m-1}\right\}>0 .
$$

Thus we can redefine $M^{a}:=M^{a, \delta}$ and $\left\{\sigma_{1}, \ldots, \sigma_{m}\right\}:=\left\{\sigma_{1}^{\prime}, \ldots, \sigma_{m}^{\prime}\right\}$.

Step 4: the desired convergence in distribution. For any $n \geq 1$ let $\widetilde{M}^{n}$ be defined by (10.3). Let $f: \mathcal{D}([0, T], X) \rightarrow[-1,1]$ be a Lipschitz function such that $|f(x)-f(y)| \leq d_{J_{1}}(x, y)$ for any $x, y \in \mathcal{D}([0, T], X)$ (see Remark 10.2). Our goal is to show that $\mathbb{E} f\left(\widetilde{M}^{n}\right) \rightarrow \mathbb{E} f(\widetilde{M})$ as $n \rightarrow \infty$.

By Step 3 we may assume that there exists $m \geq 1$ and predictable stopping times $0 \leq \sigma_{1}<\ldots<\sigma_{m} \leq T$ such that $M^{a}$ has its jumps in $\left\{\sigma_{1}, \ldots, \sigma_{m}\right\}$ satisfying (10.11), and $\Delta M_{\sigma_{1}}, \ldots, \Delta M_{\sigma_{m}}$ are simple in $L^{\infty}(\Omega ; X)$.

Let $M^{c, N_{0}}$ and $M^{q, N_{0}}$ be as in Step 1 and 2. These processes are martingales with respect to different filtrations $\mathbb{F}^{c, N_{0}}$ and $\mathbb{F}^{q, N_{0}}$ (generated by time-changed $\bar{W}_{H}$ and $\eta$ respectively). Nonetheless, if we set

$$
\mathcal{F}_{k}^{N_{0}}:=\sigma\left(\mathcal{F}_{T k / N_{0}}, \mathcal{F}_{T k / N_{0}}^{c, N_{0}}, \mathcal{F}_{T k / N_{0}}^{q, N_{0}}\right), \quad k=1, \ldots, N_{0},
$$

then $\left(d_{k}^{N_{0}}\right)_{k=1}^{N_{0}}$ defined by $(10.2)$ and

$$
\begin{aligned}
\left(e_{k}^{c, N_{0}}\right)_{k=1}^{N_{0}}:=\left(M_{T k / N_{0}}^{c, N_{0}}-M_{T k-1 / N_{0}}^{c, N_{0}}\right)_{k=1}^{N_{0}}, \quad k=1, \ldots N_{0} \\
\left(e_{k}^{q, N_{0}}\right)_{k=1}^{N_{0}}:=\left(M_{T k / N_{0}}^{q, N_{0}}-M_{T k-1 / N_{0}}^{q, N_{0}}\right)_{k=1}^{N_{0}}, \quad k=1, \ldots N_{0} \\
\left(e_{k}^{a, N_{0}}\right)_{k=1}^{N_{0}}:=\left(M_{T k / N_{0}}^{a}-M_{T k-1 / N_{0}}^{a}\right)_{k=1}^{N_{0}}, \quad k=1, \ldots N_{0}
\end{aligned}
$$

are martingale difference sequences with respect to $\mathbb{F}^{N_{0}}:=\left(\mathcal{F}_{k}^{N_{0}}\right)_{k=0}^{N_{0}}$ (since $\left.\bar{W}_{H}\right|_{[0, C k]}$ and $\left.\eta\right|_{[0, C k]}$ are independent of $\left.\bar{W}_{H}\right|_{[C k, \infty)}-\bar{W}_{H}(C k)$ and $\left.\eta\right|_{[C k, \infty)}-$ $\eta(C k))$. Let $e^{N_{0}}:=\left(e_{k}^{N_{0}}\right)_{k=1}^{N_{0}}$ be a martingale difference sequence defined by

$$
e_{k}^{N_{0}}:=e_{k}^{c, N_{0}}+e_{k}^{q, N_{0}}+e_{k}^{a, N_{0}}, \quad k=1, \ldots, N_{0} .
$$


Fix $\varepsilon>0$. Let us show that for $N_{0}$ big enough we have that for a decoupled tangent martingale difference sequence $\tilde{e}^{N_{0}}:=\left(\tilde{e}_{k}^{N_{0}}\right)_{k=1}^{N_{0}}$ the following holds true

$$
\begin{gathered}
\left|\mathbb{E} f\left(\widetilde{M}^{N_{0}}\right)-\mathbb{E} f\left(\widetilde{N}^{N_{0}}\right)\right| \lesssim_{X} \varepsilon, \\
\left|\mathbb{E} f(\widetilde{M})-\mathbb{E} f\left(\widetilde{N}^{N_{0}}\right)\right| \lesssim_{X} \varepsilon,
\end{gathered}
$$

so $\left|\mathbb{E} f(\widetilde{M})-\mathbb{E} f\left(\widetilde{M}^{N_{0}}\right)\right| \lesssim_{X} \varepsilon$ by a triangle inequality, where

$$
\widetilde{N}^{N_{0}}:=\sum_{k: T k / N_{0} \leq t} \tilde{e}_{k}^{N_{0}}, \quad t \geq 0 .
$$

First let us show (10.12). As $f$ is Lipschitz and as $M^{N_{0}}$ and $N^{N_{0}}$ are pure jump with jumps at $\left\{T / N_{0}, \ldots, T\left(N_{0}-1\right) / N_{0}\right\}$, by (10.1) it is sufficient to show that there exist a version of $\widetilde{M}^{N_{0}}$ and a version of the decoupled tangent martingale difference sequence $\tilde{e}^{N_{0}}:=\left(\tilde{e}_{k}^{N_{0}}\right)_{k=1}^{N_{0}}$ satisfying

$$
\mathbb{E} \sup _{k \geq 1}^{N_{0}}\left\|\widetilde{M}_{T k / N_{0}}^{N_{0}}-\widetilde{N}_{T k / N_{0}}^{N_{0}}\right\| \lesssim X \varepsilon
$$

Let $d^{c, N_{0}}, d^{q, N_{0}}$, and $d^{a, N_{0}}$ be defined analogously to (10.2) for martingales $M^{c}$, $M^{q}$, and $M^{a}$ respectively (note that $d^{a, N_{0}}=e^{a, N_{0}}$ ). Let $\tilde{d}^{c, N_{0}}, \tilde{d}^{q, N_{0}}$, and $\tilde{d}^{a, N_{0}}$ be the corresponding decoupled tangent martingales. Let $\tilde{e}^{c, N_{0}}, \tilde{e}^{q, N_{0}}$, and $\tilde{e}^{a, N_{0}}$ be decoupled tangent martingales to $e^{c, N_{0}}, e^{q, N_{0}}$, and $e^{a, N_{0}}$ respectively. Then by Lemma 10.5, Theorem 1.1, (10.9), and (10.10) we may find such suitable versions of $\tilde{d}^{c, N_{0}}, \tilde{d}^{q, N_{0}}, \tilde{e}^{c, N_{0}}$, and $\tilde{e}^{q, N_{0}}$, and assume that $N_{0}$ is so big that

$$
\begin{aligned}
& \mathbb{E} \sup _{k=1}^{N_{0}}\left\|\sum_{\ell=1}^{k} \tilde{e}_{\ell}^{c, N_{0}}-\tilde{d}_{\ell}^{c, N_{0}}\right\| \lesssim{ }_{X} \mathbb{E} \sup _{k=1}^{N_{0}}\left\|\sum_{\ell=1}^{k} e_{\ell}^{c, N_{0}}-d_{\ell}^{c, N_{0}}\right\| \leq \varepsilon, \\
& \mathbb{E} \sup _{k=1}^{N_{0}}\left\|\sum_{\ell=1}^{k} \tilde{e}_{\ell}^{q, N_{0}}-\tilde{d}_{\ell}^{q, N_{0}}\right\| \lesssim X \mathbb{E} \sup _{k=1}^{N_{0}}\left\|\sum_{\ell=1}^{k} e_{\ell}^{q, N_{0}}-d_{\ell}^{q, N_{0}}\right\| \leq \varepsilon .
\end{aligned}
$$

It remains to show that for $N_{0}$ big enough there exist such versions of $\tilde{d}^{a, N_{0}}$ and $\tilde{e}^{a, N_{0}}$ that

$$
\mathbb{E} \sup _{k=1}^{N_{0}}\left\|\sum_{\ell=1}^{k} \tilde{e}_{\ell}^{a, N_{0}}-\tilde{d}_{\ell}^{a, N_{0}}\right\|=0,
$$

which follows directly from the fact that $d^{a, N_{0}}=e^{a, N_{0}}$. Thus (10.15) follows from Lemma 10.5 (so that we can set $\tilde{d}^{N_{0}}:=\tilde{d}^{c, N_{0}}+\tilde{d}^{q, N_{0}}+\tilde{d}^{a, N_{0}}$ and $\tilde{e}^{N_{0}}:=$ $\left.\tilde{e}^{c, N_{0}}+\tilde{e}^{q, N_{0}}+\tilde{e}^{a, N_{0}}\right),(10.16),(10.17)$, and (10.18).

Let us show (10.13). First note that for any $N_{0}$ big enough we have that

$$
\left|\mathbb{E} f(\widetilde{M})-\mathbb{E} f\left(L^{N_{0}}\right)\right| \lesssim X \varepsilon
$$

where $L^{N_{0}}$ is a discretization of $\widetilde{M}$ defined by

$$
L_{t}^{N_{0}}:=\sum_{k: T k / N_{0} \leq t} \widetilde{M}_{T k / N_{0}}-\widetilde{M}_{T(k-1) / N_{0}}, \quad t \geq 0 .
$$


Indeed, it is sufficient to notice that a.s. as $f$ is Lipschitz

$$
\begin{aligned}
& f(\widetilde{M})-f\left(L^{N_{0}}\right) \leq d_{J_{1}}\left(\widetilde{M}, L^{N_{0}}\right) \\
& \quad \stackrel{(*)}{\leq} \sup _{t \in[0, T]}\left|\lambda^{N_{0}}(t)-t\right|+\sup _{k=1}^{N_{0}} \sup _{t=0}^{T / N_{0}} \mid \widetilde{M}_{T k / N_{0}}-\widetilde{M}_{T k / N_{0}-t} \stackrel{(* *)}{\rightarrow} 0, \quad N_{0} \rightarrow \infty,
\end{aligned}
$$

where

$$
\lambda^{N_{0}}(t):=\frac{T}{N_{0}}\left\lceil\frac{t N_{0}}{T}\right\rceil, \quad t \in[0, T]
$$

(here $\lceil a\rceil$ is the smallest integer bigger than $a \in \mathbb{R}),(*)$ follows by (10.1), and (**) follows from the fact that $\widetilde{M}$ has càdlàg paths and the fact that

$$
\sup _{t \in[0, T]}\left|\lambda^{N_{0}}(t)-t\right| \leq T / N_{0}
$$

Hence (10.19) follows by the dominated convergence theorem and therefore by (10.19), (10.1), and the fact that $f$ is Lipschitz it is sufficient to show that for any $N_{0}$ big enough there exist such a version of $\tilde{e}^{N_{0}}$ that

$$
\left|\mathbb{E} f\left(\widetilde{N}^{N_{0}}\right)-\mathbb{E} f\left(L^{N_{0}}\right)\right| \leq \mathbb{E} \sup _{k=1}^{N_{0}}\left\|\widetilde{N}_{T k / N_{0}}^{N_{0}}-\widetilde{M}_{T k / N_{0}}\right\| \lesssim X \varepsilon .
$$

By Step 1 and 2, by Lemma 10.7, and by the fact that

$$
\mathbb{E}\left(A_{T k / N_{0}}-A_{T(k-1) / N_{0}} \mid \mathcal{F}_{T(k-1) / N_{0}}\right)
$$

and

$$
\mathbb{E}\left(\nu^{j}\left(T(k-1) / N_{0}, T k / N_{0}\right] \mid \mathcal{F}_{T(k-1) / N_{0}}\right), \quad j=1, \ldots, n,
$$

are $\mathcal{F}_{T(k-1) / N_{0}}$-measurable for any $k=1, \ldots, N_{0}$, we may assume that for any $k=1, \ldots, N_{0}$

$$
\begin{aligned}
& \tilde{e}_{k}^{c, N_{0}}=\int_{C(k-1)}^{C(k-1)+\mathbb{E}\left(A_{T k / N_{0}}-A_{T(k-1) / N_{0}} \mid \mathcal{F}_{T(k-1) / N_{0}}\right)} \Phi\left(T(k-1) / N_{0}\right) \mathrm{d} \widetilde{W}_{H}, \\
& \tilde{e}_{k}^{q, N_{0}}=\int_{\cup_{j}\left[C(k-1), C(k-1)+\mathbb{E}\left(\nu^{j}\left(T(k-1) / N_{0}, T k / N_{0}\right] \mid \mathcal{F}_{T(k-1) / N_{0}}\right)\right] \times\{j\}} \mathrm{d} \bar{\eta}_{\text {ind }},
\end{aligned}
$$

where $\widetilde{W}_{H}$ and $\bar{\eta}_{\text {ind }}$ are independent copies of $W_{H}$ and $\bar{\eta}$ respectively. Moreover, by Lemma 10.5 and by the proofs of Theorem 3.18 and 3.22 we can set for any $1 \leq k \leq N_{0}$

$$
\begin{aligned}
& \widetilde{M}_{T k / N_{0}}^{c}-\widetilde{M}_{T(k-1) / N_{0}}^{c}:=\int_{C(k-1)}^{C(k-1)+A_{T k / N_{0}}-A_{T(k-1) / N_{0}}} \Phi\left(T(k-1) / N_{0}\right) \mathrm{d} \widetilde{W}_{H}, \\
& \widetilde{M}_{T k / N_{0}}^{q}-\widetilde{M}_{T(k-1) / N_{0}}^{q}:=\int_{\cup_{j}\left[C(k-1), C(k-1)+\nu^{j}\left(T(k-1) / N_{0}, T k / N_{0}\right]\right] \times\{j\}} \mathrm{d} \bar{\eta}_{\text {ind }} .
\end{aligned}
$$

Then similarly to (10.9) and (10.10) we have that for any $N_{0}$ big enough (here $\widetilde{N}^{c, N_{0}}$ and $\widetilde{N}^{q, N_{0}}$ are defined analogously to $\left.(10.14)\right)$

$$
\mathbb{E} \sup _{k=1}^{N_{0}}\left\|\widetilde{N}_{T k / N_{0}}^{c, N_{0}}-\widetilde{M}_{T k / N_{0}}^{c}\right\| \lesssim X \varepsilon
$$




$$
\mathbb{E} \sup _{k=1}^{N_{0}}\left\|\widetilde{N}_{T k / N_{0}}^{q, N_{0}}-\widetilde{M}_{T k / N_{0}}^{q}\right\| \lesssim X \varepsilon
$$

It remains to show that for $N_{0}$ big enough there exists a version $\tilde{e}^{a, N_{0}}$ such that

$$
\mathbb{E} \sup _{k=1}^{N_{0}}\left\|\widetilde{N}_{T k / N_{0}}^{a, N_{0}}-\widetilde{M}_{T k / N_{0}}^{a}\right\|=0 .
$$

To this end notice that we can choose $T$ and $N_{0}$ big enough so that $T / N_{0} \ll \delta$, and hence

$$
d_{k}^{a, N_{0}}=\Delta M_{\sigma_{\ell}}, \quad k=1, \ldots, N_{0}, \quad \ell \text { is such that } \sigma_{\ell} \in\left(T(k-1) / N_{0}, T k / N_{0}\right] .
$$

For each $k=1, \ldots, N_{0}$ set

$$
\rho_{k}:= \begin{cases}\sigma_{\ell}, & \text { if there exists } \ell \text { such that } \sigma_{\ell} \in\left(T(k-1) / N_{0}, T k / N_{0}\right] \\ T k / N_{0}, & \text { otherwise. }\end{cases}
$$

Then $\left(\rho_{k}\right)_{k=1}^{N_{0}}$ are predictable stopping times and $d_{k}^{a, N_{0}}=e_{k}^{a, N_{0}}=\Delta M_{\rho_{k}}$ a.s. for any $k=1, \ldots, N_{0}$. Let us show that we can set $\left(\tilde{e}_{k}^{a, N_{0}}\right)_{k=1}^{N_{0}}$ and $\left(\Delta \widetilde{M}_{\rho_{k}}\right)_{k=1}^{N_{0}}$ have the same distribution (and thus can be assumed to coincide). First notice that $\left(\Delta \widetilde{M}_{\rho_{k}}\right)_{k=1}^{N_{0}}$ are independent given $\mathcal{F}$. Moreover, as by [56, Lemma 25.2] $\mathcal{F}_{T(k-1) / N_{0}} \subset \mathcal{F}_{\rho_{k}} \subset \mathcal{F}_{T k / N_{0}}$ and as $e_{k}^{a, N_{0}}=\Delta M_{\rho_{k}}$ for any $k=1, \ldots, N_{0}$, for any Borel set $B \in X$ we have that

$$
\begin{aligned}
\mathbb{P} & \left(\Delta M_{\rho_{k}} \mid \mathcal{F}_{T(k-1) / N_{0}}\right)(B) \\
& =\mathbb{E}\left(\mathbf{1}_{B}\left(\Delta M_{\rho_{k}}\right) \mid \mathcal{F}_{T(k-1) / N_{0}}\right) \\
& =\mathbb{E}\left(\mathbb{E}\left(\mathbf{1}_{B}\left(\Delta M_{\rho_{k}}\right) \mid \mathcal{F}_{\rho_{k}}\right) \mid \mathcal{F}_{T(k-1) / N_{0}}\right) \\
& \stackrel{(*)}{=} \mathbb{E}\left(\mathbb{E}\left(\mathbf{1}_{B}\left(\Delta \widetilde{M}_{\rho_{k}}\right) \mid \mathcal{F}_{\rho_{k}}\right) \mid \mathcal{F}_{T(k-1) / N_{0}}\right) \\
& =\mathbb{E}\left(\mathbf{1}_{B}\left(\Delta \widetilde{M}_{\rho_{k}}\right) \mid \mathcal{F}_{T(k-1) / N_{0}}\right)=\mathbb{P}\left(\Delta \widetilde{M}_{\rho_{k}} \mid \mathcal{F}_{T(k-1) / N_{0}}\right)(B),
\end{aligned}
$$

where $(*)$ follows from Lemma 3.38. Therefore by Subsection 2.12 we can set $\tilde{e}_{k}^{a, N_{0}}:=\Delta \widetilde{M}_{\rho_{k}}$ for any $k=1, \ldots, N_{0}$, and (10.23) follows.

(10.13) follows from (10.19) and (10.20), where (10.20) holds by Lemma 10.5, (10.21), (10.22), (10.23), and the triangle inequality.

Remark 10.8. What if we endow our Skorokhod space $\mathcal{D}([0, T] ; X)$ with a different Skorokhod topology, say, with the $J_{2}, M_{1}$, or $M_{2}$ topology (see [10, 112] for the definition)? Then we will still have convergence in distribution in Theorem 10.4 as $J_{1}$ as a topology is stronger than any one of the aforementioned (see e.g. [112, Subsection 11.5.2]).

Remark 10.9. Note that Theorem 10.4 does not hold if one changes the topology of the Skorokhod space to the one generated by the sup-norm. If this is the case, then $\mathcal{D}([0, T], X)$ becomes nonseparable. In particular, if we set $T=1$ 
and $X=\mathbb{R}$, and if we choose our martingale $M$ to be a compensated standard Poisson process, then $\widetilde{M}$ has the same distribution as $M$. Let $f: \mathcal{D}([0, T], \mathbb{R}) \rightarrow$ $[-1,1]$ be a function such that

$$
f(F):=\max _{t \in[0, T] \backslash \mathbb{Q}}|\Delta F(t)| \wedge 1, \quad F \in \mathcal{D}([0, T], \mathbb{R}) .
$$

Then $f$ is Lipschitz if $\mathcal{D}([0, T], \mathbb{R})$ is endowed with the sup-norm, $\mathbb{P}(f(\widetilde{M})=$ 1) $>0$ as $\widetilde{M}$ has jumps of size 1 and as the first jump time has the exponential distribution (recall that this distribution has a density on $\mathbb{R}_{+}$thus $\mathbb{Q}$ has zero measure with this distribution), but $f\left(\widetilde{M}^{n}\right)=0$ as $\widetilde{M}^{n}$ has jumps only in rational points, so $\lim _{n \rightarrow \infty} \mathbb{E} f\left(\widetilde{M}^{n}\right) \neq \mathbb{E} f(\widetilde{M})$.

\section{Exponential formula}

In the present section we are going to provide another elementary characterization of local characteristics. Namely, we will be generalizing a Lévy-Khinchintype result for general martingales which is of the form [52, Theorem II.2.47] (see also [49]). First recall that for a given predictable stopping time $\tau$ a process $V$ is called a local martingale on $[0, \tau)$ if $V^{\tau_{n}}$ is a local martingale for any $n \geq 1$ and for any announcing sequence $\left(\tau_{n}\right)_{n \geq 1}$ of $\tau$ (see Subsection 2.4 and [52, Definition II.2.46]). Then the main result of the section is as follows.

Theorem 11.1. Let $X$ be a UMD Banach space, $M: \mathbb{R}_{+} \times \Omega \rightarrow X$ be a local martingale. Let $V$ be a càdlàg bilinear form-valued predictable process starting in zero, $\nu$ be a predictable random measure on $\mathbb{R}_{+} \times X$. Then $(V, \nu)$ are the local characteristics $\left(\llbracket M^{c} \rrbracket, \nu^{M}\right)$ of $M$ if and only if for any $x^{*} \in X^{*}$ there exists a process

$$
A_{t}\left(x^{*}\right):=-\frac{1}{2} V_{t}\left(x^{*}, x^{*}\right)+\int_{[0, t] \times X}\left(e^{i\left\langle x, x^{*}\right\rangle}-1-i\left\langle x, x^{*}\right\rangle\right) \mathrm{d} \nu(s, x), t \geq 0,
$$

such that for a process $G\left(x^{*}\right): \mathbb{R}_{+} \times \Omega \rightarrow \mathbb{R}$ defined by

$$
G_{t}\left(x^{*}\right)=\mathcal{E}\left(A\left(x^{*}\right)\right)_{t}:=e^{A_{t}\left(x^{*}\right)} \Pi_{0 \leq s \leq t}\left(1+\Delta A_{s}\left(x^{*}\right)\right) e^{-\Delta A_{s}\left(x^{*}\right)}, \quad t \geq 0,
$$

and for a predictable stopping time

$$
\tau_{G\left(x^{*}\right)}:=\inf \left\{t \geq 0: G_{t}\left(x^{*}\right)=0\right\}=\inf \left\{t \geq 0: \Delta A_{t}\left(x^{*}\right)=-1\right\},
$$

we have that

$$
t \mapsto e^{i\left\langle M_{t}, x^{*}\right\rangle} / G_{t}\left(x^{*}\right), \quad t \geq 0,
$$

is a local martingale on $\left[0, \tau_{G\left(x^{*}\right)}\right)$.

Why is Theorem 11.1 connected to the Lévy-Khinchin formula? Assume for a moment that $M$ is quasi-left continuous. Then $\nu^{M}$ is non-atomic in time, so 
$A$ does not have jumps and $G\left(x^{*}\right)=e^{A\left(x^{*}\right)}$ for any $x^{*} \in X^{*}$, and thus by Theorem 11.1 we have that $\tau_{G\left(x^{*}\right)}=\infty$, hence

$$
\begin{aligned}
t \mapsto & e^{i\left\langle M_{t}, x^{*}\right\rangle} / e^{A_{t}\left(x^{*}\right)} \\
& =e^{i\left\langle M_{t}, x^{*}\right\rangle} / e^{-\frac{1}{2} \llbracket M^{c} \rrbracket t\left(x^{*}, x^{*}\right)+\int_{[0, t] \times X}\left(e^{i\left\langle x, x^{*}\right\rangle}-1-i\left\langle x, x^{*}\right\rangle\right) \mathrm{d} \nu^{M}(s, x)}, \quad t \geq 0
\end{aligned}
$$

is a local martingale. Furthermore, if $M$ additionally has independent increments and if $M_{0}=0$, then by Corollary $9.7\left(\llbracket M^{c} \rrbracket, \nu^{M}\right)$ are deterministic, hence $A_{t}$ is deterministic, so we have that $e^{A_{t}\left(x^{*}\right)}$ is deterministic for any $t \geq 1$ and for any $x^{*} \in X^{*}$, and as $e^{i\left\langle M_{t}, x^{*}\right\rangle}$ is integrable and uniformly bounded, we have that $t \mapsto e^{i\left\langle M_{t}, x^{*}\right\rangle} / e^{A_{t}\left(x^{*}\right)}$ is a martingale, so for any $t \geq 0$ and $x^{*} \in X^{*}$

$$
\mathbb{E} e^{i\left\langle M_{t}, x^{*}\right\rangle}=e^{-\frac{1}{2} \llbracket M^{c} \rrbracket_{t}\left(x^{*}, x^{*}\right)+\int_{[0, t] \times X}\left(e^{i\left\langle x, x^{*}\right\rangle}-1-i\left\langle x, x^{*}\right\rangle\right) \mathrm{d} \nu^{M}(s, x)},
$$

which is the Lévy-Khinchin formula (see e.g. [52, 102]).

Let us shortly recall to the reader the idea of the proof of Theorem 11.1 in the real-valued setting (for the full proof we refer the reader to [52, §II.2d]). We start with proving the "only if" part, i.e. first we show that (11.3) is a local martingale given the corresponding local characteristics. Fix a local martingale $M: \mathbb{R}_{+} \times \Omega \rightarrow \mathbb{R}$ with the local characteristics $\left(\left[M^{c}\right], \nu^{M}\right)$ and fix $u \in \mathbb{R}$. Then by Itô's formula [56, Theorem 26.7] for any $t \geq 0$ we have that

$$
\begin{aligned}
e^{i u M_{t}}=1 & +i u \int_{0}^{t} e^{i u M_{s-}} \mathrm{d} M_{s}-\frac{1}{2} u^{2} \int_{0}^{t} e^{i u M_{s-}} \mathrm{d}\left[M^{c}\right]_{s} \\
& +\sum_{0 \leq s \leq t} \Delta e^{i u M_{s}}-i u e^{i u M_{s-}} \Delta M_{s} .
\end{aligned}
$$

Note that

$$
\sum_{0 \leq s \leq t} \Delta e^{i u M_{s}}=\int_{\mathbb{R}_{+} \times R} e^{i u M_{s}-\left(e^{i u x}-1\right) \mathrm{d} \mu^{M}(s, x)}
$$

and

$$
\sum_{0 \leq s \leq t} i u e^{i u M_{s-}} \Delta M_{s}=\int_{\mathbb{R}_{+} \times R} i u e^{i u M_{s-}} x \mathrm{~d} \mu^{M}(s, x),
$$

so as $\nu^{M}$ is a compensator of $\mu^{M},(11.5),(11.6)$, and (11.7) yield that

$e^{i u M_{t}}+\frac{1}{2} u^{2} \int_{0}^{t} e^{i u M_{s-}} \mathrm{d}\left[M^{c}\right]_{s}-\int_{\mathbb{R}_{+} \times R}\left(e^{i u x}-1-i u x\right) e^{i u M_{s-}} \mathrm{d} \nu^{M}(s, x), \quad t \geq 0$,

is a local martingale. Denote this local martingale by $N$ and note that

$$
N_{t}=e^{i u M_{t}}-\int_{0}^{t} e^{i u M_{s-}} \mathrm{d} A_{s}, \quad t \geq 0,
$$

where $A=A(u)$ is defined by (11.1). For simplicity also denote $Y_{t}:=e^{i u M_{t}}$ and $G_{t}:=G_{t}(u)$ for any $t \geq 0$. By a stopping time argument and thanks to the fact 
that $G$ defined by (11.2) is predictable, càdlàg, and starts in 1 , we may assume that it almost never vanishes, so $\tau_{G(u)}=\infty$, and thus we need to show that $t \mapsto Y_{t} / G_{t}$ is a local martingale in $t \geq 0$. Itô's formula [56, Theorem 26.7] yields that for any $t \geq 0$

$$
\begin{aligned}
\mathrm{d} \frac{G_{t}}{Y_{t}} & =\frac{1}{G_{t-}} \mathrm{d} Y_{t}-\frac{Y_{t-}}{G_{t-}^{2}} \mathrm{~d} G_{t}+\Delta \frac{Y_{t}}{G_{t}}-\frac{1}{G_{t-}} \Delta Y_{t}+\frac{Y_{t-}}{G_{t-}^{2}} \Delta G_{t} \\
& \stackrel{(i)}{=} \frac{1}{G_{t-}} \mathrm{d} N_{t}+\Delta \frac{Y_{t}}{G_{t}}-\frac{1}{G_{t-}} \Delta Y_{t}+\frac{Y_{t-}}{G_{t-}^{2}} \Delta G_{t} \\
& \stackrel{(i i)}{=} \frac{1}{G_{t-}} \mathrm{d} N_{t}+\frac{1}{G_{t-}\left(1+A_{t}\right)} \Delta N_{t} \Delta A_{t}
\end{aligned}
$$

where ( $i$ ) follows from (11.8) (so $\mathrm{d} Y_{t}=\mathrm{d} N_{t}+Y_{t-} \mathrm{d} A_{t}$ ) and the fact that $\mathrm{d} G_{t}=$ $G_{t-} \mathrm{d} A_{t}$ due to the definition of a stochastic exponential (see [52, Theorem I.4.61]), while (ii) follows for the similar reason and a direct computation (one needs to simply set $\Delta G_{t}=G_{t-} \Delta A_{t}$ and $\Delta Y_{t}=\Delta N_{t}+Y_{t-} \Delta A_{t}$ ). Therefore we have that $Y_{t} / G_{t}=1+\int_{0}^{t} \frac{1}{G_{s-}} \mathrm{d} N_{s}+\sum_{0 \leq s \leq t} \frac{1}{G_{s-}\left(1+A_{s}\right)} \Delta N_{s} \Delta A_{s}$, which is a local martingale as $N$ is a local martingale and thanks to [52, Proposition I.4.49].

The "if" part of Theorem 11.1 follows from the fact that as $Z:=Y / G$ is a local martingale, and as $G$ is predictable, $Y=G Z=G_{-} \cdot Z+Z Z_{-} \cdot G+[Z, G]=$ $G_{-} \cdot Z+Y_{-} \cdot A+[Z, G]$ (here we omit the difficulty with complex values, one should consider real and imaginary parts separately), where $G_{-} \cdot Z$ is a local martingale as a stochastic integral w.r.t. a local martingale and $[Z, G]$ is a local martingale by [52, Proposition I.4.49], therefore $Y-Y_{-} \cdot A$ is a local martingale, and hence $A$ has the form (11.1) with the desired $V$ and $\nu$ due to the uniqueness of a predictable finite variation compensator (see [52, Theorem I.3.18]) and the fact that $Y_{-}$has absolute value 1 , so $A$ can be reconstructed from $Y_{-} \cdot A$.

Let us finally prove Theorem 11.1. For the proof we will need the following lemma.

Lemma 11.2. Let $M: \mathbb{R}_{+} \times \Omega \rightarrow \mathbb{R}$ be a local martingale. Then we have that for any $t \geq 0$ a.s.

$$
\int_{[0, t] \times \mathbb{R}}|x|^{2} \wedge|x| \mathrm{d} \nu^{M}(x, s), \int_{[0, t] \times \mathbb{R}}|x|^{2} \wedge|x| \mathrm{d} \mu^{M}(x, s)<\infty .
$$

Proof. By a stopping time argument and by Doob's maximal inequality (2.6) we may assume that $\mathbb{E} \sup _{t \geq 0}\left|M_{t}\right|<\infty$ and that for some constant $C$ there is a.s. at most one jumps exceeding $C$ by the absolute value e.g. by setting $M:=M^{\tau_{C}}$ where $\tau_{C}:=\inf \left\{t \geq 0:\left|\Delta M_{t}\right|>C\right\}$. First let us show that

$$
\int_{[0, t] \times \mathbb{R}}|x|^{2} \mathbf{1}_{|x| \leq C} \mathrm{~d} \nu^{M}(x, s), \int_{[0, t] \times \mathbb{R}}|x|^{2} \mathbf{1}_{|x| \leq C} \mathrm{~d} \mu^{M}(x, s)<\infty,
$$

which follows from the fact that

$$
t \mapsto A_{t}:=\int_{[0, t] \times \mathbb{R}}|x|^{2} \mathbf{1}_{|x| \leq C} \mathrm{~d} \mu^{M}(x, s)=\sum_{0 \leq s \leq t}\left|\Delta M_{s}\right|^{2} \leq[M]_{t}<\infty,
$$


and $A_{t}$ locally has the first moment (so (11.10) for $\nu^{M}$ follows from (2.11)) as $A_{t}$ has jumps of at most value $C^{2}$.

Let us show that

$$
\int_{[0, t] \times \mathbb{R}}|x| \mathbf{1}_{|x|>C} \mathrm{~d} \nu^{M}(x, s), \int_{[0, t] \times \mathbb{R}}|x| \mathbf{1}_{|x|>C} \mathrm{~d} \mu^{M}(x, s)<\infty,
$$

which follows from our assumption on $M$, the fact that consequently

$$
\mathbb{E} \int_{[0, t] \times \mathbb{R}}|x| \mathbf{1}_{|x|>C} \mathrm{~d} \mu^{M}(x, s)=\mathbb{E} \sum_{0 \leq s \leq t}\left|\Delta M_{s}\right| \mathbf{1}_{\left|\Delta M_{s}\right|>C} \leq 2 \mathbb{E} \sup _{t \geq 0}\left|M_{t}\right|<\infty,
$$

and from (2.11). (11.9) follows from (11.10) and (11.11).

Proof of Theorem 11.1. Let us start with the "only if" part. Let $(V, \nu)=$ $\left(\llbracket M^{c} \rrbracket, \nu^{M}\right)$. Notice that for any $x^{*} \in X^{*}$ by Taylor's formula

$$
e^{i\left\langle x, x^{*}\right\rangle}-1-i\left\langle x, x^{*}\right\rangle \approx \frac{1}{2}\left|\left\langle x, x^{*}\right\rangle\right|^{2}, \quad x \in X,
$$

for small $\left\langle x, x^{*}\right\rangle$ and

$$
\left|e^{i\left\langle x, x^{*}\right\rangle}-1-i\left\langle x, x^{*}\right\rangle\right| \leq\left|\left\langle x, x^{*}\right\rangle\right|+2 \lesssim\left|\left\langle x, x^{*}\right\rangle\right|, \quad x \in X,
$$

for big $\left\langle x, x^{*}\right\rangle$, so by Lemma 11.2 and A.2 the integral

$$
\int_{[0, t] \times X} e^{i\left\langle x, x^{*}\right\rangle}-1-i\left\langle x, x^{*}\right\rangle \mathrm{d} \nu^{M}(s, x), \quad t \geq 0,
$$

is well defined. Consequently, $A$ is well defined.

Now let us show that (11.3) is a local martingale. As by Definition 2.12 and by Remark 2.16 we have that a.s.

$$
\llbracket M^{c} \rrbracket\left(x^{*}, x^{*}\right)=\left[\left\langle M^{c}, x^{*}\right\rangle\right]_{t}=\left[\left\langle M, x^{*}\right\rangle^{c}\right]_{t}, \quad t \geq 0,
$$

and since by Lemma A.2 we have that

$\int_{[0, t] \times X} e^{i\left\langle x, x^{*}\right\rangle}-1-i\left\langle x, x^{*}\right\rangle \mathrm{d} \nu^{M}(s, x)=\int_{[0, t] \times \mathbb{R}}\left(e^{i r}-1-i r\right) \mathrm{d} \nu^{\left\langle M, x^{*}\right\rangle}(s, r), \quad t \geq 0$,

we can restrict ourselves to the one dimensional setting. Let $X=\mathbb{R}, M$ be one dimensional. We will be using the setting of [52, Section II.2]. Let $h(r)=r$, $r \in \mathbb{R}$ (though $h$ is assumed to be bounded in [52, Section II.2], in our case both $\int\left(e^{i r}-1-i h(r)\right) \mathrm{d} \nu^{\left\langle M, x^{*}\right\rangle}(s, r)$ and $\int h \mathrm{~d} \bar{\mu}^{M}$ are well defined by (11.12) and by Theorem 3.30, so we can set $h$ to be as defined). Then we have that the representation of $M$ given by formula [52, II.2.35] is

$$
M=M_{0}+M^{c}+\int h \mathrm{~d} \bar{\mu}^{M}+\int_{[0, \cdot] \times \mathbb{R}} r-h(r) \mathrm{d} \mu^{M}(s, r)+B=M^{c}+\int h \mathrm{~d} \bar{\mu}^{M},
$$


so by the uniqueness of this representation and by [52, Theorem II.2.47] the desired follows.

Let us now show the "if" part. It is sufficient to show that $V\left(x^{*}, x^{*}\right)=$ $\llbracket M^{c} \rrbracket\left(x^{*}, x^{*}\right)$ a.s. for any $x^{*} \in X^{*}$, and analogously to the proof of Theorem 7.3 it is sufficient to show that $\nu^{x^{*}}=\nu^{\left\langle M, x^{*}\right\rangle}$ for any $x^{*} \in X^{*}$, where $\nu^{x^{*}}$ is defined as a unique predictable random measure on $\mathbb{R}_{+} \times \mathbb{R}$ such that for any elementary predictable $F: \mathbb{R}_{+} \times \Omega \times \mathbb{R} \rightarrow \mathbb{R}_{+}$one has that a.s.

$$
\int_{\mathbb{R}_{+} \times Y} F(s, \cdot, y) \mathrm{d} \nu^{x^{*}}(s, \cdot, y)=\int_{\mathbb{R}_{+} \times X} F\left(s, \cdot,\left\langle x, x^{*}\right\rangle\right) \mathrm{d} \nu(s, \cdot, x) .
$$

In order to construct such a random measure it is sufficient to set a.s.

$$
\int \mathbf{1}_{A}(s, \cdot) \mathbf{1}_{B}(y) \mathrm{d} \nu^{x^{*}}(s, \cdot, y):=\int \mathbf{1}_{A}(s, \cdot) \mathbf{1}_{B}\left(\left\langle x, x^{*}\right\rangle\right) \mathrm{d} \nu(s, \cdot, x)
$$

for any $A \in \mathcal{P}$ and $B \in \mathcal{B}(\mathbb{R})$. To this end note that $V\left(x^{*}, x^{*}\right)=\llbracket M^{c} \rrbracket\left(x^{*}, x^{*}\right)$ a.s. for any $x^{*} \in X^{*}$ as well as $\nu^{x^{*}}=\nu^{\left\langle M, x^{*}\right\rangle}$ a.s. by the fact that (11.3) is a local martingale and by [52, Theorem II.2.47 and II.2.49].

Remark 11.3. Note that $\tau_{G\left(\varepsilon x^{*}\right)} \rightarrow \infty$ a.s. as $\varepsilon \rightarrow 0$ for any $x^{*} \in X^{*} \backslash\{0\}$. Indeed, due to the definition of $\tau_{G\left(\varepsilon x^{*}\right)}$ is it sufficient to show that

$$
\int_{[0, t] \times X}\left|e^{i\left\langle x, \varepsilon x^{*}\right\rangle}-1-i\left\langle x, \varepsilon x^{*}\right\rangle\right| \mathrm{d} \nu^{M}(s, x) \rightarrow 0, \quad \varepsilon \rightarrow 0,
$$

which follows from the fact that for $\varepsilon<1$

$$
\left|e^{i\left\langle x, \varepsilon x^{*}\right\rangle}-1-i\left\langle x, \varepsilon x^{*}\right\rangle\right| \lesssim\left|\left\langle x, \varepsilon x^{*}\right\rangle\right|^{2} \wedge\left|\left\langle x, \varepsilon x^{*}\right\rangle\right| \lesssim \varepsilon\left(\left|\left\langle x, x^{*}\right\rangle\right|^{2} \wedge\left|\left\langle x, x^{*}\right\rangle\right|\right)
$$

and from Lemma 11.2. It remains unknown for the author whether $\tau_{G\left(x^{*}\right)} \rightarrow \infty$ as $x^{*} \rightarrow 0$.

Remark 11.4. Let $M$ have independent increments and let $M_{0}=0$. In this case one can show that analogously to (11.4)

$$
\mathbb{E} e^{i\left\langle M_{t}, x^{*}\right\rangle}=G_{t}\left(x^{*}\right)=e^{A_{t}\left(x^{*}\right)} \Pi_{0 \leq s \leq t}\left(1+\Delta A_{s}\left(x^{*}\right)\right) e^{-\Delta A_{s}\left(x^{*}\right)},
$$

for any $t \geq 0$ and $x^{*} \in X^{*}$ even if $t>\tau_{G\left(x^{*}\right)}$. First note that $\tau_{G\left(x^{*}\right)}$ in this case is a deterministic stopping time as $G\left(x^{*}\right)$ is deterministic by Corollary 9.7. Second, in order to prove (11.13) for $t \geq \tau_{G\left(x^{*}\right)}$ (the case $t<\tau_{G\left(x^{*}\right)}$ follows from Theorem 11.1) let $r=\tau_{G\left(x^{*}\right)}$. Then by the discussions in Subsection 3.6

$$
\begin{aligned}
0=1+\Delta A_{r}\left(x^{*}\right) & =1+\int_{\mathbb{R}_{+} \times X} \mathbf{1}_{\{r\}}(s)\left(e^{i\left\langle x, x^{*}\right\rangle}-1-i\left\langle x, x^{*}\right\rangle\right) \mathrm{d} \nu^{M}(s, x) \\
& =1+\mathbb{E}\left(e^{i\left\langle\Delta M_{r}, x^{*}\right\rangle}-1-i\left\langle\Delta M_{r}, x^{*}\right\rangle \mid \mathcal{F}_{r-}\right) \\
& =\mathbb{E} e^{i\left\langle\Delta M_{r}, x^{*}\right\rangle},
\end{aligned}
$$


where the latter follows as $M$ has independent increments and as $M$ is a martingale (so $\left.\mathbb{E}\left(\left\langle\Delta M_{r}, x^{*}\right\rangle \mid \mathcal{F}_{r-}\right)=0\right)$. Therefore $\mathbb{E} e^{i\left\langle\Delta M_{r}, x^{*}\right\rangle}=0$ and as $M$ has independent increments

$$
\mathbb{E} e^{i\left\langle M_{t}, x^{*}\right\rangle}=\mathbb{E} e^{i\left\langle M_{r-}, x^{*}\right\rangle} \mathbb{E} e^{i\left\langle\Delta M_{r}, x^{*}\right\rangle} \mathbb{E} e^{i\left\langle\left(M_{t}-M_{r}\right), x^{*}\right\rangle}=0,
$$

so (11.13) is satisfied. Note that thanks to the techniques from Section 9 one can omit the UMD assumption.

\section{Characteristic subordination and characteristic domination of martingales}

In the present section we prove basic $L^{p}$-estimates concerning characteristic subordination and characteristic domination.

\subsection{Characteristic subordination}

Let $X$ be a Banach space, $M, N: \mathbb{R}_{+} \times \Omega \rightarrow X$ be local martingales. Then $N$ is called to be weakly differentially subordinate to $M$ if $\left|\left\langle N_{0}, x^{*}\right\rangle\right| \leq\left|\left\langle M_{0}, x^{*}\right\rangle\right|$ a.s. and if $\left[\left\langle M, x^{*}\right\rangle\right]_{t}-\left[\left\langle N, x^{*}\right\rangle\right]_{t}$ is nondecreasing a.s. for any $x^{*} \in X^{*}$. Weak differential subordination was intensively studied during past two years in [91, $113,115,116,117,119]$. In particular, it was shown in $[119$, Subsection 7.4$]$ that if this is the case and $X$ is UMD then

$$
\mathbb{E} \sup _{t \geq 0}\left\|N_{t}\right\|^{p} \lesssim p, X \mathbb{E} \sup _{t \geq 0}\left\|M_{t}\right\|^{p} .
$$

In the present section we will consider a predictable analogue of weak differential subordination which exploits local characteristics - characteristic subordination.

Definition 12.1. Let $X$ be a Banach space, $M, N: \mathbb{R}_{+} \times \Omega \rightarrow X$ be local martingales. Then $N$ is characteristicly subordinate to $M$ if for any $x^{*} \in X^{*}$ a.s.

(A) $\left|\left\langle N_{0}, x^{*}\right\rangle\right| \leq\left|\left\langle M_{0}, x^{*}\right\rangle\right|$,

(B) $\left[\left\langle N, x^{*}\right\rangle^{c}\right]_{t}-\left[\left\langle N, x^{*}\right\rangle^{c}\right]_{s} \leq\left[\left\langle M, x^{*}\right\rangle^{c}\right]_{t}-\left[\left\langle M, x^{*}\right\rangle^{c}\right]_{s}$,

(C) $\nu^{\left\langle N, x^{*}\right\rangle} \leq \nu^{\left\langle M, x^{*}\right\rangle}$.

(Recall that though $M$ and $N$ take their values in a general (not necessarily UMD) Banach space $X$ (so $M^{c}$ and $N^{c}$ may not have sense), $\left\langle M, x^{*}\right\rangle$ and $\left\langle N, x^{*}\right\rangle$ are real-valued martingales, so $\left\langle M, x^{*}\right\rangle^{c}$ and $\left\langle N, x^{*}\right\rangle^{c}$ exist, see Theorem 2.18).

Note that if $M$ and $N$ are continuous, then $N$ is characteristically subordinate to $M$ if and only if $N$ is weakly differentially subordinate to $M$. The following two propositions show that weak differential subordination coincides with characteristic subordination only in continuous case.

Proposition 12.2. Weak differential subordination does not imply characteristic subordination. 
Proof. Let $M$ be a purely discontinuous nonzero martingale with an a.s. finite measure $\nu^{M}$ (e.g. a compensated standard Poisson process which stopped at time point 1) and $N=\frac{1}{2} M$. Then $N$ is weakly differentially subordinate to $M$, but is not characteristically subordinate. Indeed, by Lemma A.2 we have that a.s. for any Borel $B \in X$

$$
\nu^{N}([0, t] \times B)=\nu^{M}([0, t] \times 2 B), \quad t \geq 0 .
$$

Thus we have that a.s. $\nu^{M}([0, t] \times X \backslash\{0\})=\nu^{N}([0, t] \times X \backslash\{0\})$, and as $\nu^{M}$ is finite, by (12.2) we have that $\nu^{N} \nless \leq \nu^{M}$ on a set of positive probability, as if we assume the converse, then for the sets $C_{n}=2^{n} B \backslash 2^{n-1} B,-\infty<n<\infty$, where $B \in X$ is the unit ball, we have that $C_{n}=2 C_{n-1}$, and hence by $(12.2)$ for any $-\infty<n<\infty$

$$
\nu^{M}\left([0, t] \times C_{n}\right) \geq \nu^{N}\left([0, t] \times C_{n}\right)=\nu^{M}\left([0, t] \times C_{n+1}\right), \quad t \geq 0,
$$

so $\nu^{M}$ is infinite (as $C_{n}$ 's are disjoint, $\cup C_{n}=X \backslash\{0\}$, and as $\nu^{M} \neq 0$, there exists $n$ and $t$ such that $\left.\nu^{M}\left([0, t] \times C_{n}\right)>0\right)$, which contradicts our assumption.

Proposition 12.3. Characteristic subordination does not imply weak differential subordination.

Proof. It is sufficient to consider two independent compensated standard Poisson processes $\widetilde{N}_{1}$ and $\widetilde{N}_{2}$, as they are characteristically subordinate to each other (because they have the same local characteristics), but they are not weakly differentially subordinate to each other as they have jumps at different times a.s., i.e. $\Delta \widetilde{N}_{1} \neq 0 \Rightarrow \Delta \widetilde{N}_{2}=0$ and $\Delta \widetilde{N}_{2} \neq 0 \Rightarrow \Delta \widetilde{N}_{1}=0$ a.s. for any $t \geq 0$.

Remark 12.4. What do the aforementioned examples demonstrate? These examples show that $N$ is weakly differentially subordinate to $M$ if $N$ has smaller jumps than $M$, and $N$ is characteristically subordinate to $M$ if $N$ has the same jumps as $M$ but these jumps occur less often.

Let us now formulate the main theorem of the present section.

Theorem 12.5. Let $X$ be a Banach space. Then $X$ is UMD if and only if for any $1 \leq p<\infty$ and for any local martingales $M, N: \mathbb{R}_{+} \times \Omega \rightarrow X$ such that $N$ is characteristicly subordinate to $M$ one has that

$$
\mathbb{E} \sup _{t \geq 0}\left\|N_{t}\right\|^{p} \lesssim_{p, X} \mathbb{E} \sup _{t \geq 0}\left\|M_{t}\right\|^{p}
$$

The proof of the theorem is based on the canonical decomposition (see Subsection 2.7) and treating each case of the canonical decomposition separately. Therefore we will need the following propositions.

Proposition 12.6. Let $X$ be a UMD Banach space, $(J, \mathcal{J})$ be a measurable space, $\mu$ and $\mu^{\prime}$ be quasi-left continuous optional random measures on $\mathbb{R}_{+} \times J$ such that for the corresponding compensators $\nu$ and $\nu^{\prime}$ we have that $\nu^{\prime} \leq \nu$ 
a.s. Then for any elementary predictable $F: \mathbb{R}_{+} \times \Omega \times J \rightarrow X$ and for any $1 \leq p<\infty$ we have that

$$
\mathbb{E} \sup _{t \geq 0}\left\|\int_{[0, t] \times J} F \mathrm{~d} \bar{\mu}^{\prime}\right\|^{p} \lesssim p, X \mathbb{E} \sup _{t \geq 0}\left\|\int_{[0, t] \times J} F \mathrm{~d} \bar{\mu}\right\|^{p},
$$

where $\bar{\mu}:=\mu-\nu$ and $\bar{\mu}^{\prime}:=\mu^{\prime}-\nu^{\prime}$.

Proof. Let $\mu_{\text {Cox }}$ and $\mu_{\text {Cox }}^{\prime}$ be Cox processes directed by $\nu$ and $\nu^{\prime}$ respectively, and set $\bar{\mu}_{\text {Cox }}:=\mu_{\text {Cox }}-\nu, \bar{\mu}_{\text {Cox }}^{\prime}:=\mu_{\text {Cox }}^{\prime}-\nu^{\prime}$. Then by Theorem 3.22

$$
\begin{aligned}
& \mathbb{E} \sup _{t \geq 0}\left\|\int_{[0, t] \times J} F \mathrm{~d} \bar{\mu}\right\|^{p} \bar{\sim}_{p, X} \mathbb{E} \mathbb{E}_{\text {Cox }}\left\|\int_{\mathbb{R}+\times J} F \mathrm{~d} \bar{\mu}_{\text {Cox }}\right\|^{p}, \\
& \mathbb{E} \sup _{t \geq 0}\left\|\int_{[0, t] \times J} F \mathrm{~d} \bar{\mu}^{\prime}\right\|^{p} \bar{\sim}_{p, X} \mathbb{E} \mathbb{E}_{\text {Cox }}\left\|\int_{\mathbb{R}_{+} \times J} F \mathrm{~d} \bar{\mu}_{\text {Cox }}^{\prime}\right\|^{p},
\end{aligned}
$$

where $\mathbb{E}_{\text {Cox }}$ is defined in Example 2.5. Thus it is sufficient to show that for a.e. fixed $\omega \in \Omega$

$$
\mathbb{E}_{\text {Cox }}\left\|\int_{\mathbb{R}_{+} \times J} F \mathrm{~d} \bar{\mu}_{\text {Cox }}^{\prime}\right\|^{p} \leq \mathbb{E}_{\text {Cox }}\left\|\int_{\mathbb{R}_{+} \times J} F \mathrm{~d} \bar{\mu}_{\text {Cox }}\right\|^{p} .
$$

Let us now show (12.3). Fix $\omega \in \Omega$ such that $\nu^{\prime}(\omega) \leq \nu(\omega)$. Then both $\mu_{\text {Cox }}$ and $\mu_{\text {Cox }}^{\prime}$ are time changed Poisson. Let $\nu^{\prime \prime}=\nu-\nu^{\prime}, \mu_{\text {Cox }}^{\prime \prime}$ be the Cox process directed by $\nu^{\prime \prime}$. As $\omega$ is fixed, $\mu_{\text {Cox }}^{\prime}$ and $\mu_{\text {Cox }}^{\prime \prime}$ are independent and $\mu_{\text {Cox }}^{\prime}+\mu_{\text {Cox }}^{\prime \prime}$ has the same compensator and hence coincides in distribution with $\mu_{\text {Cox }}$ so we can set $\mu_{\text {Cox }}=\mu_{\text {Cox }}^{\prime}+\mu_{\text {Cox }}^{\prime \prime}, \bar{\mu}_{\text {Cox }}=\bar{\mu}_{\text {Cox }}^{\prime}+\bar{\mu}_{\text {Cox }}^{\prime \prime}$. Therefore (12.3) follows from the fact that for a fixed $\omega \in \Omega$ the process $F$ is deterministic, the fact that a conditional expectation operator is a contraction (see [46, Section 2.6]), and

$$
\int_{\mathbb{R}_{+} \times J} F \mathrm{~d} \bar{\mu}_{\text {Cox }}^{\prime}=\mathbb{E}\left(\int_{\mathbb{R}_{+} \times J} F \mathrm{~d} \bar{\mu}_{\text {Cox }} \mid \sigma\left(\bar{\mu}_{\text {Cox }}^{\prime}\right)\right),
$$

as $\mu_{\text {Cox }}^{\prime}$ and $\mu_{\text {Cox }}^{\prime \prime}$ are independent for any fixed $\omega \in \Omega$.

We will also need the following proposition which is some sense extends stochastic domination inequality [96, Theorem 2] (see also [79]).

Proposition 12.7. Let $X$ be a Banach space, $\left(\xi_{n}\right)_{n=1}^{N}$ and $\left(\xi_{n}^{\prime}\right)_{n=1}^{N}$ be independent $X$-valued symmetric random variable such that for any Borel set $A \subset X \backslash\{0\}$ and for any $n=1, \ldots, N$ one has that $\mathbb{P}\left(\xi_{n}^{\prime} \in A\right) \leq \mathbb{P}\left(\xi_{n} \in A\right)$. Then for any convex symmetric function $\phi: X \rightarrow \mathbb{R}_{+}$one has that

$$
\mathbb{E} \phi\left(\sum_{n=1}^{N} \xi_{n}^{\prime}\right) \leq \mathbb{E} \phi\left(\sum_{n=1}^{N} \xi_{n}\right) .
$$

Proof. As $\left(\xi_{n}\right)_{n=1}^{N}$ and $\left(\xi_{n}^{\prime}\right)_{n=1}^{N}$ are symmetric, (12.4) is equivalent to

$$
\mathbb{E} \phi\left(\sum_{n=1}^{N} r_{n} \xi_{n}^{\prime}\right) \leq \mathbb{E} \phi\left(\sum_{n=1}^{N} r_{n} \xi_{n}\right),
$$


where $\left(r_{n}\right)_{n=1}^{N}$ is an independent sequence of i.i.d. Rademachers (see Definition 2.1). Thus it is sufficient to show (12.5). By an approximation argument we may assume that $\left(\xi_{n}\right)_{n=1}^{N}$ and $\left(\xi_{n}^{\prime}\right)_{n=1}^{N}$ take finitely many values. By the assumption of the proposition we have that for any $n=1, \ldots, N$ the random variable $\xi_{n}^{\prime}$ has the same distribution as $\eta_{n}\left(\xi_{n}\right) \xi_{n}$, where for any $x \in X \backslash\{0\}$ we define a random variable $\eta_{n}(x)$ on an independent probability space $(\widetilde{\Omega}, \widetilde{\mathcal{F}}, \widetilde{\mathbb{P}})$ to be such that $\eta_{n}(x) \in\{0,1\}$ a.s. and $\mathbb{E} \eta_{n}(x)=\frac{\mathbb{P}\left(\xi_{n}^{\prime}=x\right)}{\mathbb{P}\left(\xi_{n}=x\right)}$, where we set $\frac{0}{0}:=0$. Fix $\omega \in \Omega$ and $\widetilde{\omega} \in \widetilde{\Omega}$. Then in order to show (12.5) it remains to prove that

$$
\mathbb{E}_{r} \phi\left(\sum_{n=1}^{N} r_{n} \eta_{n}(x)(\widetilde{\omega}) \xi_{n}(\omega)\right) \leq \mathbb{E} \phi\left(\sum_{n=1}^{N} r_{n} \xi_{n}(\omega)\right),
$$

and as all the coefficients $\left(\eta_{n}(x)(\widetilde{\omega})\right)_{n=1}^{N}$ are either 0 or 1 , the latter follows from Jensen's inequality (see [46, Proposition 2.6.29]) as $\sum_{n=1}^{N} r_{n} \eta_{n}(x)(\widetilde{\omega}) \xi_{n}(\omega)$ is just a conditional expectation of $\sum_{n=1}^{N} r_{n} \xi_{n}(\omega)$ given $\sigma\left(r_{n} \eta_{n}(x)(\widetilde{\omega})\right)_{n=1}^{N}$.

Proof of Theorem 12.5. Without loss of generality (as $\left\|N_{0}\right\| \leq\left\|M_{0}\right\|$, see [115, Lemma 3.6]) we can set $M_{0}=N_{0}=0$. Let $M=M^{c}+M^{q}+M^{a}$ and $N=$ $N^{c}+N^{q}+N^{a}$ be the canonical decompositions. Note that due to Definition 12.1 and Subsection 3.2 we have that $\nu^{\left\langle N^{q}, x^{*}\right\rangle} \leq \nu^{\left\langle M^{q}, x^{*}\right\rangle}$ and $\nu^{\left\langle N^{a}, x^{*}\right\rangle} \leq \nu^{\left\langle M^{a}, x^{*}\right\rangle}$ a.s. for any $x^{*} \in X^{*}$, so $N^{i}$ is characteristically subordinate to $M^{i}$ for any $i \in\{c, q, a\}$. By (2.9) it is sufficient to show that

$$
\begin{aligned}
& \mathbb{E} \sup _{t \geq 0}\left\|N_{t}^{c}\right\|^{p} \lesssim p, X \mathbb{E} \sup _{t \geq 0}\left\|M_{t}^{c}\right\|^{p}, \\
& \mathbb{E} \sup _{t \geq 0}\left\|N_{t}^{q}\right\|^{p} \lesssim p, X \mathbb{E} \sup _{t \geq 0}\left\|M_{t}^{q}\right\|^{p}, \\
& \mathbb{E} \sup _{t \geq 0}\left\|N_{t}^{a}\right\|^{p} \lesssim p, X \mathbb{E} \sup _{t \geq 0}\left\|M_{t}^{a}\right\|^{p} .
\end{aligned}
$$

First of all, (12.6) follows from (12.1). (12.7) follows from Proposition 12.6, the fact that $M^{q}=\int x \mathrm{~d} \bar{\mu}^{M^{q}}$ and $N^{q}=\int x \mathrm{~d} \bar{\mu}^{N^{q}}$ by Theorem 3.30, and the fact that $N^{q}$ is characteristically subordinate to $M^{q}$. Finally, (12.8) follows from a standard approximation argument (see e.g. Proposition B.1), the fact that any purely discontinuous martingale with finitely many predictable jumps has a discrete representation (see e.g. the proof of Proposition 3.37), Proposition 12.7, the construction of a decoupled tangent martingale from the proof of Theorem 3.39, the symmetrization argument (see the proof of Theorem 5.9), and the fact that $N^{a}$ is characteristically subordinate to $M^{a}$.

\subsection{Characteristic domination}

We can straighten characteristic subordination in the following way. Let $X$ be a Banach space, $M$ and $N$ be $X$-valued martingales. Then $N$ is characteristically dominated by $M$ if a.s. $\left|\left\langle N_{0}, x^{*}\right\rangle\right| \leq\left|\left\langle M_{0}, x^{*}\right\rangle\right|$ for any $x^{*} \in X^{*}, \llbracket N^{c} \rrbracket_{\infty} \leq \llbracket M^{c} \rrbracket_{\infty}$ and $\nu^{N}\left(\mathbb{R}_{+} \times \cdot\right) \leq \nu^{M}\left(\mathbb{R}_{+} \times \cdot\right)$. Then the following theorem holds true. 
Theorem 12.8. Let $X$ be a Banach space. Then $X$ has the UMD property if and only if for any (equivalently, for some) $1 \leq p<\infty$ and for any $X$ valued quasi-left continuous local martingales such that $N$ is characteristically dominated by $M$ one has that

$$
\mathbb{E} \sup _{0 \leq t<\infty}\left\|N_{t}\right\|^{p} \lesssim p, X \mathbb{E} \sup _{0 \leq t<\infty}\left\|M_{t}\right\|^{p} .
$$

For the proof we will need the following proposition.

Proposition 12.9. Let $X$ be a UMD Banach space, $(J, \mathcal{J})$ be a measurable space, $\mu$ and $\mu^{\prime}$ be optional quasi-left continuous random measures on $\mathbb{R}_{+} \times J$ such that for the corresponding compensators $\nu$ and $\nu^{\prime}$ we have that $\nu^{\prime}\left(\mathbb{R}_{+} \times A\right) \leq$ $\nu\left(\mathbb{R}_{+} \times A\right)<\infty$ a.s. for any $A \in \mathcal{J}$. Then for any elementary $\mathcal{B}\left(\mathbb{R}_{+}\right) \otimes \mathcal{F}_{0} \otimes J_{-}$ measurable $F: \mathbb{R}_{+} \times \Omega \times J \rightarrow X$ and for any $1 \leq p<\infty$ we have that

$$
\mathbb{E} \sup _{t \geq 0}\left\|\int_{[0, t] \times J} F \mathrm{~d} \bar{\mu}^{\prime}\right\|^{p} \lesssim p, X \mathbb{E} \sup _{t \geq 0}\left\|\int_{[0, t] \times J} F \mathrm{~d} \bar{\mu}\right\|^{p},
$$

where $\bar{\mu}:=\mu-\nu$ and $\bar{\mu}^{\prime}:=\mu^{\prime}-\nu^{\prime}$.

Proof. Without loss of generality we may assume that $t \mapsto F(t, \cdot, \cdot)$ is a constant a.e. on $\Omega \times J$ as otherwise we just approximate $F$ by a step $\mathcal{F}_{0}$-measurable function and apply the whole proof below for each step of $F$ separately.

The proposition follows analogously Proposition 12.6, but then we need to show (12.3) in a difference way. Fix $\omega \in \Omega$ such that

$$
\nu^{\prime}\left(\mathbb{R}_{+} \times A\right) \leq \nu\left(\mathbb{R}_{+} \times A\right)<\infty, \quad A \in \mathcal{J} .
$$

Then by the definition of a stochastic integral (2.10), by the fact that $F$ is elementary predictable, and by the definition of a Cox process (see Subsubsection 3.4.1) there exist $x_{1}, \ldots, x_{M} \in X$, independent Poisson random variables $\left(\xi_{m}\right)_{m=1}^{M}$ with parameters $\left(\lambda_{m}\right)_{m=1}^{M}$ and independent Poisson random variables $\left(\xi_{m}^{\prime}\right)_{m=1}^{M}$ with parameters $\left(\lambda_{m}^{\prime}\right)_{m=1}^{M}$ satisfying $\lambda_{m}^{\prime} \leq \lambda_{m}$ for any $m=1, \ldots, M$ by (12.10) such that

$$
\begin{aligned}
& \int_{[0, t] \times J} F \mathrm{~d} \bar{\mu}_{\text {Cox }}=\sum_{m=1}^{M} x_{m}\left(\xi_{m}-\lambda_{m}\right), \\
& \int_{[0, t] \times J} F \mathrm{~d} \bar{\mu}_{\text {Cox }}^{\prime}=\sum_{m=1}^{M} x_{m}\left(\xi_{m}^{\prime}-\lambda_{m}^{\prime}\right)
\end{aligned}
$$

(see e.g. [56, p. 88] or [7, Section 23] for details on Poisson distributions). Now by the fact that sum of two independent Poisson random variable is again has Poisson distribution with parameter being the sum of the corresponding parameters and by independence of all $\xi_{m}$ 's and $\xi_{m}^{\prime}$ 's we can assume that there exists a sequence of independent Poisson random variables $\left(\xi_{m}^{\prime \prime}\right)_{m=1}^{M}$ with parameters $\left(\lambda_{m}^{\prime \prime}\right)_{m=1}^{M}=\left(\lambda_{m}-\lambda_{m}^{\prime}\right)_{m=1}^{M}$ such that $\xi_{m}-\lambda_{m}=\left(\xi_{m}^{\prime}-\lambda_{m}^{\prime}\right)+\left(\xi_{m}^{\prime \prime}-\lambda_{m}^{\prime \prime}\right)$, and then the desired follows from the same conditional expectation trick used in the end of the proof of Proposition 12.6 and Theorem 3.22. 
Proof of Theorem 12.8. The "if" part follows directly from Proposition 3.17 as the latter is a particular case of Theorem 12.8.

Let us show the "only if" part. By Proposition 3.17 we have that for the continuous terms of the Meyer-Yoeurp decompositions $M=M^{c}+M^{d}$ and $N=N^{c}+N^{d}$ (see Remark 2.19)

$$
\mathbb{E} \sup _{0 \leq t<\infty}\left\|N_{t}^{c}\right\|^{p} \lesssim_{p, X} \mathbb{E} \sup _{0 \leq t<\infty}\left\|M_{t}^{c}\right\|^{p} .
$$

Also note that as $M$ and $N$ are quasi-left continuous, $M^{d}=M^{q}$ and $N^{d}=N^{q}$ (see Subsection 2.7 for the definition of $M^{q}$ and $N^{q}$ ). Thus by Theorem 2.18, by Proposition 3.17, and by the considerations above it is sufficient to show (12.9) for $M$ and $N$ being purely discontinuous quasi-left continuous. First let us show that if

$$
\mathbb{E} \sup _{0 \leq t<\infty}\left\|M_{t}\right\|^{p}<\infty
$$

then $\nu^{M}\left(\mathbb{R}_{+} \times \bar{B}\right)<\infty$ a.s. for any centered ball $B \subset X$ (here $\bar{B}$ is the complement of $B$ ). Indeed, as $M$ is purely discontinuous, then by the fact that any UMD Banach space has a finite Gaussian cotype $q \geq 2$ (see [47, Definition 7.1.17, Corollary 7.2.11, and Proposition 7.3.15]) and by Burkholder-Davis-Gundy inequalities [119, Subsection 6.1] for a family $\left(\gamma_{t}\right)_{t \geq 0}$ of i.i.d. standard Gaussians and for any $\delta>0$ we have that

$$
\begin{aligned}
\mathbb{E} \sup _{0 \leq t<\infty}\left\|M_{t}\right\|^{p} & \bar{\sim}_{p, X} \mathbb{E}_{\gamma}\left\|\sum_{t \geq 0} \gamma_{t} \Delta M_{t}\right\|^{p} \geq \mathbb{E} \mathbb{E}_{\gamma}\left\|\sum_{t \geq 0} \gamma_{t} \Delta M_{t} \mathbf{1}_{\left\|\Delta M_{t}\right\|>\delta}\right\|^{p}, \\
& \stackrel{(i)}{\sim}_{p, q} \mathbb{E}\left(\mathbb{E}_{\gamma}\left\|\sum_{t \geq 0} \gamma_{t} \Delta M_{t} \mathbf{1}_{\left\|\Delta M_{t}\right\|>\delta}\right\|^{q}\right)^{p / q} \\
& \stackrel{(i i)}{=} \mathbb{E}\left(\mathbb{E}_{\gamma}\left\|\int_{\mathbb{R}_{+} \times X} \gamma_{t} x \mathbf{1}_{\|x\|>\delta} \mathrm{d} \mu^{M}(t, x)\right\|^{q}\right)^{p / q} \\
& \stackrel{(i i i)}{{ }^{(i i)}} \underset{X}{ } \mathbb{E}\left(\int_{\mathbb{R}_{+} \times X}\|x\|^{q} \mathbf{1}_{\|x\|>\delta} \mathrm{d} \mu^{M}(t, x)\right)^{p / q} \\
& \gtrsim_{\delta} \mathbb{E}\left(\int_{\mathbb{R}_{+} \times X} \mathbf{1}_{\|x\|>\delta} \mathrm{d} \mu^{M}(t, x)\right)^{p / q},
\end{aligned}
$$

where $\mathbb{E}_{\gamma}$ is defined by Example 2.5, (i) follows from Kahane-Khinchin inequalities [47, Theorem 6.2.6], (ii) holds by the definition of $\mu^{M}$ (see (2.14)), and (iii) follows from the definition of a Gaussian cotype [47, Definition 7.1.17]. Therefore we have that

$$
\int_{\mathbb{R}_{+} \times X} \mathbf{1}_{\|x\|>\delta} \mathrm{d} \mu^{M}(t, x) \text { is finite a.s., }
$$

and hence its compensator,

$$
\int_{\mathbb{R}_{+} \times X} \mathbf{1}_{\|x\|>\delta} \mathrm{d} \nu^{M}(t, x)=\nu^{M}\left(\mathbb{R}_{+} \times \bar{B}\right) \text { is finite a.s. }
$$


as well because for stopping times

$$
\tau_{n}:=\inf \left\{\int_{[0, t] \times X} \mathbf{1}_{\|x\|>\delta} \mathrm{d} \mu^{M}(\cdot, x)>n\right\}
$$

we have that by (12.12) $\left\{\tau_{n}=\infty\right\} \nearrow \Omega$ up to a negligible set, and then (12.13) follows by [52, Theorem I.3.17].

Now let $M^{m}$ and $N^{m}$ be as defined by (B.6). Then by Proposition 12.9, by the definition of the characteristic domination, by Theorem 3.30, and by (12.13) we have that

$$
\mathbb{E} \sup _{0 \leq t<\infty}\left\|N_{t}^{m}\right\|^{p} \lesssim p, X \mathbb{E} \sup _{0 \leq t<\infty}\left\|M_{t}^{m}\right\|^{p},
$$

and thus

$$
\mathbb{E} \sup _{0 \leq t<\infty}\left\|N_{t}^{m}\right\|^{p} \lesssim_{p, X} \mathbb{E} \sup _{0 \leq t<\infty}\left\|M_{t}^{m}\right\|^{p},
$$

holds true by Proposition B.2 and by letting $m \rightarrow \infty$. Then (12.9) follows from $(12.11),(12.14),(2.9)$, and the fact that $M$ and $N$ are quasi-left continuous.

Remark 12.10. It is not known whether Theorem 12.8 holds for general local martingales. By Theorem 3.39 and by Proposition B.1 the main issue here is in proving a similar statement for discrete martingales with independent increments. Let us state this problem here as open. Let $X$ be a Banach space, $1 \leq p<\infty$. Let $\left(\xi_{n}\right)_{n \geq 1}$ and $\left(\eta_{n}\right)_{n \geq 1}$ be $X$-valued mean-zero independent random variables such that for any Borel $B \in X \backslash\{0\}$

$$
\sum_{n} \mathbb{P}\left(\xi_{n} \in B\right) \leq \sum_{n} \mathbb{P}\left(\eta_{n} \in B\right) .
$$

Does there exists a constant $C$ (perhaps depending on $p$ and $X)$ such that

$$
\mathbb{E}\left\|\sum_{n} \xi_{n}\right\|^{p} \leq C \mathbb{E}\left\|\sum_{n} \eta_{n}\right\|^{p} ?
$$

By a standard symmetrization trick [66, Lemma 6.3] one can assume that $\xi_{n}$ 's and $\eta_{n}$ 's are symmetric. But even the symmetric case is not known for the author.

\section{Appendix A: Tangency under linear operators}

The goal of this section is to show that $T M$ and $T N$ are tangent for any linear operator $T$ from a certain family given $M$ and $N$ are tangent. Let us start with bounded linear operators between Banach spaces.

Theorem A.1. Let $X$ be a Banach space, $M, N: \mathbb{R}_{+} \times \Omega \rightarrow X$ be tangent local martingales. Let $Y$ be a Banach space, $T \in \mathcal{L}(X, Y)$. Then $T M$ and $T N$ have local characteristics and are tangent. Moreover, if $N$ is a decoupled tangent local martingale to $M$, then $T N$ is a decoupled tangent local martingale to $T M$. 
The proof needs the following lemma.

Lemma A.2. Let $X, Y$, and $Z$ be Banach spaces, $M: \mathbb{R}_{+} \times \Omega \rightarrow X$ be a local martingale, $T \in \mathcal{L}(X, Y)$. Then for any predictable function $F: \mathbb{R}_{+} \times \Omega \times Y \rightarrow Z$ we have that

$$
\int_{[0, \cdot] \times Y}\|F(s, \cdot, y)\| \mathrm{d} \mu^{T M}(s, y),
$$

is locally finite if and only if

$$
\int_{[0, \cdot] \times X}\|F(s, \cdot, T x)\| \mathrm{d} \mu^{M}(s, x),
$$

and if this is the case then

$$
\int_{[0, \cdot] \times X} F(s, \cdot, y) \mathrm{d} \mu^{T M}(s, y)=\int_{[0, \cdot] \times X} F(s, \cdot, T x) \mathrm{d} \mu^{M}(s, x) .
$$

Moreover, if

$$
\mathbb{E} \int_{[0, t] \times Y}\|F(s, \cdot, y)\| \mathrm{d} \mu^{T M}(s, y)<\infty
$$

or, equivalently,

$$
\mathbb{E} \int_{[0, t] \times X}\|F(s, \cdot, T x)\| \mathrm{d} \mu^{M}(s, x)<\infty
$$

for any $t \geq 0$, then

$$
\begin{aligned}
\int_{[0, t] \times Y} F(s, \cdot, y) & \mathrm{d} \nu^{T M}(s, y) \\
& =\int_{[0, t] \times X} F(s, \cdot, T x) \mathrm{d} \nu^{M}(s, x)<\infty, \quad t \geq 0 .
\end{aligned}
$$

Proof. The first part of the lemma follows directly from the definition of $\mu^{M}$ and $\mu^{T M}$ (see (2.14)). (A.1) follows for a similar reason. (A.2) follows from (A.1), the definition of a compensator random measure (see Subsection 2.8), the definition of a compensator process [52, Theorem I.3.17], and the uniqueness of the compensator process.

Proof of Theorem A.1. Let us start with the first part of the theorem. We need to show that $T M$ and $T N$ have local characteristics which coincide. First let us show that $T M$ and $T N$ have the Meyer-Yoeurp decomposition. As $M$ and $N$ are tangent, they have the Meyer-Yoeurp decomposition (see Subsection 3.1). Let $M=M^{c}+M^{d}$ and $N=N^{c}+N^{d}$ be this decomposition. Then $T M=$ $T M^{c}+T M^{d}$ and $T N=T N^{c}+T N^{d}$ are the Meyer-Yoeurp decomposition as well since $T M^{c}$ and $T N^{c}$ are continuous and $T M^{d}$ and $T N^{d}$ are purely discontinuous as for any $y^{*} \in Y^{*}$ we have that both $\left\langle T M^{d}, y^{*}\right\rangle=\left\langle M^{d}, T^{*} y^{*}\right\rangle$ and $\left\langle T N^{d}, y^{*}\right\rangle=\left\langle N^{d}, T^{*} y^{*}\right\rangle$ are purely discontinuous (see Definition 2.14). 
Let us show that both $\llbracket T M^{c} \rrbracket$ and $\llbracket T N^{c} \rrbracket$ exist and coincide. To this end it is sufficient to notice that for any $y^{*} \in Y^{*}$ we have that a.s.

$$
\begin{aligned}
\llbracket T M^{c} \rrbracket_{t}\left(y^{*}, y^{*}\right)= & {\left[\left\langle T M^{c}, y^{*}\right\rangle\right]_{t}=\left[\left\langle M^{c}, T^{*} y^{*}\right\rangle\right]_{t} } \\
& \leq\left\|\llbracket M^{c} \rrbracket_{t}\right\|\left\|T^{*} y^{*}\right\|^{2} \leq\left\|\llbracket M^{c} \rrbracket_{t}\right\|\|T\|^{2}\left\|y^{*}\right\|^{2}, \quad t \geq 0,
\end{aligned}
$$

where we define $\|V\|:=\sup _{z^{*} \in Z^{*},\left\|z^{*}\right\| \leq 1} V\left(z^{*}, z^{*}\right)$ for any symmetric bilinear form $V: Z^{*} \times Z^{*} \rightarrow \mathbb{R}$ for any Banach space $Z$. Therefore $\left\|\llbracket T M^{c} \rrbracket_{t}\right\| \leq$ $\|T\|^{2}\left\|\llbracket M^{c} \rrbracket_{t}\right\|$ for any $t \geq 0$, and $\llbracket T M^{c} \rrbracket_{t}$ defines a bounded bilinear form. The same holds for $\llbracket T N^{c} \rrbracket_{t}$. Equality $\llbracket T M^{c} \rrbracket_{t}=\llbracket T N^{c} \rrbracket_{t}$ follows directly from the fact that for any $y^{*} \in Y^{*}$ a.s. for any $t \geq 0$ by (A.3)

$$
\llbracket T M^{c} \rrbracket_{t}\left(y^{*}, y^{*}\right)=\llbracket M^{c} \rrbracket_{t}\left(T^{*} y^{*}, T^{*} y^{*}\right)=\llbracket N^{c} \rrbracket_{t}\left(T^{*} y^{*}, T^{*} y^{*}\right)=\llbracket T N^{c} \rrbracket_{t}\left(y^{*}, y^{*}\right) .
$$

The fact that $\nu^{T M}=\nu^{T N}$ a.s. follows from Lemma A.2. Therefore $T M$ and $T N$ have the same local characteristics, and thus are tangent.

Let us show the second part of the theorem. This part follows from Definition 3.3 and the fact that action of a bounded linear operator does not ruin independence and martingality (so if $N(\omega)$ is a martingale with independent increments, $T N(\omega)$ is so as well).

Another important type of operators are stopping time operators. Apparently, they also preserve tangency.

Theorem A.3. Let $X$ be a Banach space, $M, N: \mathbb{R}_{+} \times \Omega \rightarrow X$ be tangent local martingales. Then $M^{\tau}$ and $N^{\tau}$ are tangent. Moreover, if $N$ is a decoupled tangent local martingale to $M$, and if $\tau$ is an $\mathbb{F}$-stopping time (where $\mathbb{F}$ is the original filtration where $M$ used to live), then $N^{\tau}$ is a decoupled tangent local martingale to $M^{\tau}$.

Proof. Let $M=M^{c}+M^{d}$ and $N=N^{c}+N^{d}$ be the Meyer-Yoeurp decompositions. Then $M^{\tau}=\left(M^{c}\right)^{\tau}+\left(M^{d}\right)^{\tau}$ is the Meyer-Yoeurp decomposition as $\left\langle M^{\tau}, x^{*}\right\rangle=\left\langle\left(M^{c}\right)^{\tau}, x^{*}\right\rangle+\left\langle\left(M^{d}\right)^{\tau}, x^{*}\right\rangle$ is the Meyer-Yoeurp decomposition since $\left\langle\left(M^{c}\right)^{\tau}, x^{*}\right\rangle=\left\langle M^{c}, x^{*}\right\rangle^{\tau}$ is continuous and since $\left\langle\left(M^{d}\right)^{\tau}, x^{*}\right\rangle=\left\langle M^{d}, x^{*}\right\rangle^{\tau}$ is purely discontinuous by [56, Theorem 26.6]. For the same reason $N^{\tau}=$ $\left(N^{c}\right)^{\tau}+\left(N^{d}\right)^{\tau}$ is the Meyer-Yoeurp decomposition as well. Moreover, by [56, Theorem 26.6] we have that $\llbracket\left(M^{c}\right)^{\tau} \rrbracket=\llbracket M^{c} \rrbracket^{\tau}=\llbracket N^{c} \rrbracket^{\tau}=\llbracket\left(N^{c}\right)^{\tau} \rrbracket$ a.s. It remains to show that $\nu^{M^{\tau}}=\nu^{N^{\tau}}$. To this end it is sufficient to notice that $\mu^{M^{\tau}}=\mathbf{1}_{[0, \tau]} \mu^{M}$ and $\mu^{N^{\tau}}=\mathbf{1}_{[0, \tau]} \mu^{N}$, so by [52, Proposition II.1.30] (see also $\left[32\right.$, Subsection 5.4]) we have that $\nu^{M^{\tau}}=\mathbf{1}_{[0, \tau]} \nu^{M}=\mathbf{1}_{[0, \tau]} \nu^{N}=\nu^{N^{\tau}}$, so $M^{\tau}$ and $N^{\tau}$ are tangent.

Let us show the second part. First recall that by Definition $3.3 \mathrm{~N}$ is a decoupled tangent local martingale if and only if $N(\omega)$ is a martingale with independent increments with local characteristics $\left(\llbracket M^{c}(\omega) \rrbracket, \nu^{M}(\omega)\right)$ for any $\omega \in \Omega$ (where $\Omega, \mathcal{F}, \mathbb{P}$ is a probability space where $M$ lives). As $\tau$ is an $\mathbb{F}$-stopping time, it depends only on $\omega, N^{\tau}(\omega)=N(\omega)^{\tau(\omega)}$ is a martingale with independent increments having $\left(\llbracket M^{c}(\omega) \rrbracket^{\tau(\omega)}, \mathbf{1}_{[0, \tau(\omega)]} \nu^{M}(\omega)\right)$ as its local characteristics, so the desired holds true. 


\section{Appendix B: Martingale approximations}

Here we present certain martingales approximation techniques shown in [119]. Recall that a function $\phi: \mathbb{R}_{+} \rightarrow \mathbb{R}_{+}$is called to have a moderate growth if there exists $\alpha>0$ such that $\phi(2 t) \leq \alpha \phi(t)$ for any $t \geq 0$.

\section{B.1. Purely discontinuous martingales with accessible jumps}

Let $X$ be a Banach space, $M: \mathbb{R}_{+} \times \Omega \rightarrow X$ be a purely discontinuous martingale with accessible jumps. Then by Lemma 2.20 there exist finite predictable stopping times $\left(\tau_{n}\right)_{n \geq 1}$ with disjoint graphs which exhaust jumps of $M$. For any $m \geq 1$ let us define

$$
M_{t}^{m}:=\sum_{n=1}^{m} \Delta M_{\tau_{n}} \mathbf{1}_{\left[\tau_{n}, \infty\right)}(t) \quad t \geq 0 .
$$

Then due to Lemma $2.11 M^{m}$ is a local martingale for any $m \geq 1$ and by [119, Subsubsection 7.5.2] the following proposition holds true.

Proposition B.1. Let $X$ be a UMD Banach space, $\phi: \mathbb{R}_{+} \rightarrow \mathbb{R}_{+}$be a convex function of moderate growth with $\phi(0)=0, M: \mathbb{R}_{+} \times \Omega \rightarrow X$ be a purely discontinuous martingale with accessible jumps such that

$$
\mathbb{E} \sup _{t \geq 0} \phi\left(\left\|M_{t}\right\|\right)<\infty
$$

For any $m \geq 1$ let $M^{m}$ be defined by (B.1). Then

$$
\mathbb{E} \sup _{t \geq 0} \phi\left(\left\|M_{t}^{m}\right\|\right)<\infty, \quad m \geq 1
$$

and moreover

$$
\mathbb{E} \sup _{t \geq 0} \phi\left(\left\|M_{t}-M_{t}^{m}\right\|\right) \rightarrow 0, \quad m \rightarrow \infty .
$$

Proof. The case of $\phi(t)=t^{p}, 1 \leq p<\infty$, was covered [119, Subsubsection 7.5.2]. The general case follows analogously. Indeed, first notice that $\llbracket M^{m} \rrbracket_{\infty} \leq$ $\llbracket M \rrbracket_{\infty}$ a.s. for any $m \geq 1$ by [56, Theorem 26.6 and Corollary 26.15]. Thus $\gamma\left(\llbracket M^{m} \rrbracket_{\infty}\right) \leq \gamma\left(\llbracket M \rrbracket_{\infty}\right)$ by [119, Subsection 3.2], so by [119, Section 5] we have that

$$
\begin{aligned}
\mathbb{E} \sup _{t \geq 0} \phi\left(\left\|M_{t}^{m}\right\|\right) \bar{\sim}_{\phi, X} \mathbb{E} \phi\left(\gamma\left(\llbracket M^{m} \rrbracket_{\infty}\right)\right) & \\
& \leq \mathbb{E} \phi\left(\gamma\left(\llbracket M \rrbracket_{\infty}\right)\right) \bar{\sim}_{p, X} \mathbb{E} \sup _{t \geq 0} \phi\left(\left\|M_{t}\right\|\right)<\infty,
\end{aligned}
$$

and (B.3) holds true. Moreover, by [119, Subsubsection 7.5.2] we know that $\llbracket M-M^{m} \rrbracket_{\infty} \rightarrow 0$ monotonically a.s., so by $[119$, Subsection 3.2$]$ we have that 
$\gamma\left(\llbracket M-M^{m} \rrbracket_{\infty}\right) \rightarrow 0$ monotonically a.s., and hence by the dominated convergence theorem, the fact that $\llbracket M-M^{m} \rrbracket_{\infty} \leq \llbracket M \rrbracket_{\infty}$ a.s., by (B.2), and [119, Section 5 and Subsection 3.2] we have that

$$
\mathbb{E} \sup _{t \geq 0} \phi\left(\left\|M-M_{t}^{m}\right\|\right) \bar{\sim}_{\phi, X} \mathbb{E} \phi\left(\gamma\left(\llbracket M-M^{m} \rrbracket_{\infty}\right)\right) \rightarrow 0, \quad m \rightarrow \infty,
$$

so (B.4) follows.

\section{B.2. Purely discontinuous quasi-left continuous martingales}

Now let $M: \mathbb{R}_{+} \times \Omega \rightarrow X$ be a purely discontinuous quasi-left continuous martingale. Then by Theorem 3.30 (see also [32, Subsection 5.4] and [119, Subsection 7.2 and 7.5]) $M_{t}=\int_{[0, t] \times X} x \mathrm{~d} \bar{\mu}^{M}$, where $\mu^{M}$ is as defined by (2.14), $\nu^{M}$ is the corresponding compensator, $\bar{\mu}^{M}=\mu^{M}-\nu^{M}$. For each $m \geq 1$ let

$$
M_{t}^{m}:=\int_{[0, t] \times X} x \mathbf{1}_{[0, m]}(\|x\|) \mathrm{d} \bar{\mu}^{M}, \quad t \geq 0,
$$

or

$$
M_{t}^{m}:=\int_{[0, t] \times X} x \mathbf{1}_{[1 / m, \infty)}(\|x\|) \mathrm{d} \bar{\mu}^{M}, \quad t \geq 0 .
$$

Then due to [119, Subsubsection 7.5.1] and [32] $M^{m}$ is a local martingale and the following proposition holds true by [119, Subsubsection 7.5.1].

Proposition B.2. Let $X$ be a UMD Banach space, $\phi: \mathbb{R}_{+} \rightarrow \mathbb{R}_{+}$be a convex function of moderate growth with $\phi(0)=0, M: \mathbb{R}_{+} \times \Omega \rightarrow X$ be a purely discontinuous quasi-left continuous martingale such that

$$
\mathbb{E} \sup _{t \geq 0} \phi\left(\left\|M_{t}\right\|\right)<\infty
$$

For any $m \geq 1$ let $M^{m}$ be defined by (B.5) or (B.6). Then

$$
\mathbb{E} \sup _{t \geq 0} \phi\left(\left\|M_{t}^{m}\right\|\right)<\infty, \quad m \geq 1,
$$

and moreover

$$
\mathbb{E} \sup _{t \geq 0} \phi\left(\left\|M_{t}-M_{t}^{m}\right\|\right) \rightarrow 0, \quad m \rightarrow \infty .
$$

Proof. The proof is fully analogous to the proof of Proposition B.1.

\section{Acknowledgment}

The author thanks Sjoerd Dirksen, Stefan Geiss, Jan van Neerven, and Mark Veraar for fruitful discussions and helpful comments. The author is grateful to the reviewer for their careful reading and detailed comments, which helped to significantly improve the presentation of the paper. 


\section{References}

[1] O.O. Aalen and J.M. Hoem. Random time changes for multivariate counting processes. Scand. Actuar. J., (2):81-101, 1978. MR0503082

[2] D. Applebaum. Lévy processes and stochastic integrals in Banach spaces. Probab. Math. Statist., 27(1):75-88, 2007. MR2353272

[3] D. Applebaum and M. Riedle. Cylindrical Lévy processes in Banach spaces. Proc. Lond. Math. Soc. (3), 101(3):697-726, 2010. MR2734958

[4] K.B. Athreya and J.R. Peters. Continuity of translation operators. Proc. Amer. Math. Soc., 139(11):4027-4040, 2011. MR2823048

[5] C. Bélisle, J.-C. Massé, and T. Ransford. When is a probability measure determined by infinitely many projections? Ann. Probab., 25(2):767-786, 1997. MR1434125

[6] P. Billingsley. Convergence of probability measures. John Wiley \& Sons, Inc., New York-London-Sydney, 1968. MR0233396

[7] P. Billingsley. Probability and measure. Wiley Series in Probability and Mathematical Statistics. John Wiley \& Sons, Inc., New York, third edition, 1995. A Wiley-Interscience Publication. MR1324786

[8] V.I. Bogachev. Gaussian measures, volume 62 of Mathematical Surveys and Monographs. American Mathematical Society, Providence, RI, 1998. MR1642391

[9] V.I. Bogachev. Measure theory. Vol. I, II. Springer-Verlag, Berlin, 2007. MR2267655

[10] B. Böttcher. Embedded Markov chain approximations in Skorokhod topologies. Probab. Math. Statist., 39(2):259-277, 2019. MR4053319

[11] J. Bourgain. Some remarks on Banach spaces in which martingale difference sequences are unconditional. Ark. Mat., 21(2):163-168, 1983. MR0727340

[12] T.C. Brown and M.G. Nair. A simple proof of the multivariate random time change theorem for point processes. J. Appl. Probab., 25(1):210-214, 1988. MR0929518

[13] Z. Brzeźniak and E. Hausenblas. Maximal regularity for stochastic convolutions driven by Lévy processes. Probab. Theory Related Fields, 145(34):615-637, 2009. MR2529441

[14] D.L. Burkholder. Distribution function inequalities for martingales. Ann. Probability, 1:19-42, 1973. MR0365692

[15] D.L. Burkholder. A geometrical characterization of Banach spaces in which martingale difference sequences are unconditional. Ann. Probab., 9(6):997-1011, 1981. MR0632972

[16] D.L. Burkholder. A geometric condition that implies the existence of certain singular integrals of Banach-space-valued functions. In Conference on harmonic analysis in honor of Antoni Zygmund, Vol. I, II (Chicago, Ill., 1981), Wadsworth Math. Ser., pages 270-286. Wadsworth, Belmont, CA, 1983. MR0730072

[17] D.L. Burkholder. Boundary value problems and sharp inequalities for martingale transforms. Ann. Probab., 12(3):647-702, 1984. MR0744226 
[18] D.L. Burkholder. Martingales and Fourier analysis in Banach spaces. In Probability and analysis (Varenna, 1985), volume 1206 of Lecture Notes in Math., pages 61-108. Springer, Berlin, 1986. MR0864712

[19] D.L. Burkholder. Sharp inequalities for martingales and stochastic integrals. Astérisque, (157-158):75-94, 1988. Colloque Paul Lévy sur les Processus Stochastiques (Palaiseau, 1987). MR0976214

[20] D.L. Burkholder. Martingales and singular integrals in Banach spaces. In Handbook of the geometry of Banach spaces, Vol. I, pages 233-269. NorthHolland, Amsterdam, 2001. MR1863694

[21] D.L. Burkholder, B.J. Davis, and R.F. Gundy. Integral inequalities for convex functions of operators on martingales. pages 223-240, 1972. MR0400380

[22] D.R. Cox. Some statistical methods connected with series of events. J. Roy. Statist. Soc. Ser. B., 17:129-157; discussion, 157-164, 1955. MR0092301

[23] D.R. Cox and V. Isham. Point processes. Chapman \& Hall, LondonNew York, 1980. Monographs on Applied Probability and Statistics. MR0598033

[24] S.G. Cox and S. Geiss. On decoupling in Banach spaces. arXiv:1805.12377, 2018.

[25] S.G. Cox and M.C. Veraar. Some remarks on tangent martingale difference sequences in $L^{1}$-spaces. Electron. Comm. Probab., 12:421-433, 2007. MR2350579

[26] S.G. Cox and M.C. Veraar. Vector-valued decoupling and the BurkholderDavis-Gundy inequality. Illinois J. Math., 55(1):343-375 (2012), 2011. MR3006692

[27] G. Da Prato and J. Zabczyk. Stochastic equations in infinite dimensions, volume 152 of Encyclopedia of Mathematics and its Applications. Cambridge University Press, Cambridge, second edition, 2014. MR3236753

[28] V.H. de la Peña. A bound on the moment generating function of a sum of dependent variables with an application to simple random sampling without replacement. Ann. Inst. H. Poincaré Probab. Statist., 30(2):197211, 1994. MR1276997

[29] V.H. de la Peña and E. Giné. Decoupling. Probability and its Applications (New York). Springer-Verlag, New York, 1999. From dependence to independence, Randomly stopped processes. $U$-statistics and processes. Martingales and beyond. MR1666908

[30] S. Dirksen. Itô isomorphisms for $L^{p}$-valued Poisson stochastic integrals. Ann. Probab., 42(6):2595-2643, 2014. MR3265175

[31] S. Dirksen, C. Marinelli, and I.S. Yaroslavtsev. Stochastic evolution equations in $L^{p}$-spaces driven by jump noise. In preparation.

[32] S. Dirksen and I.S. Yaroslavtsev. $L^{q}$-valued Burkholder-Rosenthal inequalities and sharp estimates for stochastic integrals. Proc. Lond. Math. Soc. (3), 119(6):1633-1693, 2019.

[33] R.M. Dudley. Real analysis and probability, volume 74 of Cambridge Studies in Advanced Mathematics. Cambridge University Press, Cambridge, 
2002. Revised reprint of the 1989 original. MR1932358

[34] Yu. Eidelman, V. Milman, and A. Tsolomitis. Functional analysis, volume 66 of Graduate Studies in Mathematics. American Mathematical Society, Providence, RI, 2004. An introduction. MR2067694

[35] D. Filipović, S. Tappe, and J. Teichmann. Jump-diffusions in Hilbert spaces: existence, stability and numerics. Stochastics, 82(5):475-520, 2010. MR2739608

[36] D.J.H. Garling. Brownian motion and UMD-spaces. In Probability and Banach spaces (Zaragoza, 1985), volume 1221 of Lecture Notes in Math., pages 36-49. Springer, Berlin, 1986. MR0875006

[37] D.J.H. Garling. Random martingale transform inequalities. In Probability in Banach spaces 6 (Sandbjerg, 1986), volume 20 of Progr. Probab., pages 101-119. Birkhäuser Boston, Boston, MA, 1990. MR1056706

[38] S. Geiss. A counterexample concerning the relation between decoupling constants and UMD-constants. Trans. Amer. Math. Soc., 351(4):13551375, 1999. MR1458301

[39] S. Geiss, S. Montgomery-Smith, and E. Saksman. On singular integral and martingale transforms. Trans. Amer. Math. Soc., 362(2):553-575, 2010. MR2551497

[40] S. Geiss and I.S. Yaroslavtsev. Dyadic and stochastic shifts and Volterratype operators. In preparation.

[41] B. Grigelionis. The representation of integer-valued random measures as stochastic integrals over the Poisson measure. Litovsk. Mat. Sb., 11:93108, 1971. MR0293703

[42] B. Grigelionis. Martingale characterization of random processes with independent increments. Litovsk. Mat. Sb., 17(1):75-86, 212, 1977. MR0451416

[43] E. Hausenblas. Maximal inequalities of the Itô integral with respect to Poisson random measures or Lévy processes on Banach spaces. Potential Anal., 35(3):223-251, 2011. MR2832576

[44] P. Hitczenko. On tangent sequences of UMD-space valued random vectors. Unpublished manuscript.

[45] P. Hitczenko. Comparison of moments for tangent sequences of random variables. Probab. Theory Related Fields, 78(2):223-230, 1988. MR0945110

[46] T.P. Hytönen, J.M.A.M. van Neerven, M.C. Veraar, and L. Weis. Analysis in Banach spaces. Vol. I. Martingales and Littlewood-Paley theory, volume 63 of Ergebnisse der Mathematik und ihrer Grenzgebiete. Springer, 2016. MR3617205

[47] T.P. Hytönen, J.M.A.M. van Neerven, M.C. Veraar, and L. Weis. Analysis in Banach spaces. Vol. II. Probabilistic methods and operator theory, volume 67 of Ergebnisse der Mathematik und ihrer Grenzgebiete. 3. Folge. A Series of Modern Surveys in Mathematics. Springer, 2017. MR3752640

[48] J. Jacod. Calcul stochastique et problèmes de martingales, volume 714 of Lecture Notes in Mathematics. Springer, Berlin, 1979. MR0542115

[49] J. Jacod. Processus à accroissements indépendants: une condition nécessaire et suffisante de convergence en loi. Z. Wahrsch. Verw. Gebiete, 63(1):109-136, 1983. MR0699790 
[50] J. Jacod. Une généralisation des semimartingales: les processus admettant un processus à accroissements indépendants tangent. In Seminar on probability, XVIII, volume 1059 of Lecture Notes in Math., pages 91-118. Springer, Berlin, 1984. MR0770952

[51] J. Jacod and H. Sadi. Processus admettant un processus à accroissements indépendants tangent: cas général. In Séminaire de Probabilités, XXI, volume 1247 of Lecture Notes in Math., pages 479-514. Springer, Berlin, 1987. MR0942000

[52] J. Jacod and A.N. Shiryaev. Limit theorems for stochastic processes, volume 288 of Grundlehren der Mathematischen Wissenschaften. SpringerVerlag, Berlin, second edition, 2003. MR1943877

[53] A. Jakubowski. On the Skorokhod topology. Ann. Inst. H. Poincaré Probab. Statist., 22(3):263-285, 1986. MR0871083

[54] A. Jakubowski, S. Kwapień, P.R. de Fitte, and J. Rosiński. Radonification of cylindrical semimartingales by a single Hilbert-Schmidt operator. Infin. Dimens. Anal. Quantum Probab. Relat. Top., 5(3):429-440, 2002. MR1930962

[55] W.B. Johnson and J. Lindenstrauss, editors. Handbook of the geometry of Banach spaces. Vol. 2. North-Holland, Amsterdam, 2003. MR1999613

[56] O. Kallenberg. Foundations of modern probability. Probability and its Applications (New York). Springer-Verlag, New York, second edition, 2002. MR1876169

[57] O. Kallenberg. Random measures, theory and applications, volume 77 of Probability Theory and Stochastic Modelling. Springer, Cham, 2017. MR3642325

[58] O. Kallenberg. Tangential existence and comparison, with applications to single and multiple integration. Probab. Math. Statist., 37(1):21-52, 2017. MR3652201

[59] N.J. Kalton, E. Lorist, and L. Weis. Euclidean structures and operator theory in Banach spaces. arXiv:1912.09347, 2019.

[60] I. Karatzas and S.E. Shreve. Brownian motion and stochastic calculus, volume 113 of Graduate Texts in Mathematics. Springer-Verlag, New York, second edition, 1991. MR1121940

[61] J.F.C. Kingman. Poisson processes, volume 3 of Oxford Studies in Probability. The Clarendon Press, Oxford University Press, New York, 1993. Oxford Science Publications. MR1207584

[62] S. Kwapień and W. A. Woyczyński. Tangent sequences of random variables: basic inequalities and their applications. In Almost everywhere convergence (Columbus, OH, 1988), pages 237-265. Academic Press, Boston, MA, 1989. MR1035249

[63] S. Kwapień and W.A. Woyczyński. Decoupling of martingale transforms and stochastic integrals for processes with independent increments. In Probability theory and harmonic analysis (Cleveland, Ohio, 1983), volume 98 of Monogr. Textbooks Pure Appl. Math., pages 139-148. Dekker, New York, 1986. MR0830235

[64] S. Kwapień and W.A. Woyczyński. Semimartingale integrals via decou- 
pling inequalities and tangent processes. Probab. Math. Statist., 12(2):165200 (1992), 1991. MR1199772

[65] S. Kwapień and W.A. Woyczyński. Random series and stochastic integrals: single and multiple. Probability and its Applications. Birkhäuser Boston, Inc., Boston, MA, 1992. MR1167198

[66] M. Ledoux and M. Talagrand. Probability in Banach spaces. Classics in Mathematics. Springer-Verlag, Berlin, 2011. Isoperimetry and processes, Reprint of the 1991 edition. MR2814399

[67] E. Lenglart. Relation de domination entre deux processus. Ann. Inst. H. Poincaré Sect. B (N.S.), 13(2):171-179, 1977. MR0471069

[68] N. Lindemulder, M.C. Veraar, and I.S. Yaroslavtsev. The UMD property for Musielak-Orlicz spaces. In Positivity and noncommutative analysis, Trends Math., pages 349-363. Birkhäuser/Springer, Cham, [2019] (C2019. MR4042282

[69] C. Marinelli. On maximal inequalities for purely discontinuous $L_{q}$-valued martingales. arXiv:1311.7120, 2013.

[70] C. Marinelli, C. Prévôt, and M. Röckner. Regular dependence on initial data for stochastic evolution equations with multiplicative Poisson noise. J. Funct. Anal., 258(2):616-649, 2010. MR2557949

[71] C. Marinelli and M. Röckner. On maximal inequalities for purely discontinuous martingales in infinite dimensions. In Séminaire de Probabilités XLVI, volume 2123 of Lecture Notes in Math., pages 293-315. Springer, 2014. MR3330821

[72] C. Marinelli and M. Röckner. On the maximal inequalities of Burkholder, Davis and Gundy. Expo. Math., 34(1):1-26, 2016. MR3463679

[73] T.R. McConnell. Decoupling and stochastic integration in UMD Banach spaces. Probab. Math. Statist., 10(2):283-295, 1989. MR1057936

[74] M. Métivier. Semimartingales, volume 2 of de Gruyter Studies in Mathematics. Walter de Gruyter \& Co., Berlin-New York, 1982. A course on stochastic processes. MR0688144

[75] M. Métivier and J. Pellaumail. Stochastic integration. Academic Press [Harcourt Brace Jovanovich, Publishers], New York-London-Toronto, Ont., 1980. Probability and Mathematical Statistics. MR0578177

[76] P.-A. Meyer. Un cours sur les intégrales stochastiques. Springer, Berlin, 1976. MR0501332

[77] P.A. Meyer. Démonstration simplifiée d'un théorème de Knight. pages 191-195. Lecture Notes in Math., Vol. 191, 1971. MR0380972

[78] P.A. Meyer. Notes sur les intégrales stochastiques. I. Intégrales hilbertiennes. In Séminaire de Probabilités, XI (Univ. Strasbourg, Strasbourg, 1975/1976), pages 446-462. Lecture Notes in Math., Vol. 581. Springer, Berlin, 1977. MR0501333

[79] S.J. Montgomery-Smith and A.R. Pruss. A comparison inequality for sums of independent random variables. J. Math. Anal. Appl., 254(1):35-42, 2001. MR1807885

[80] J.M.A.M. van Neerven. $\gamma$-radonifying operators - a survey. In The AMSI$A N U$ Workshop on Spectral Theory and Harmonic Analysis, volume 44 of 
Proc. Centre Math. Appl. Austral. Nat. Univ., pages 1-61. Austral. Nat. Univ., Canberra, 2010. MR2655391

[81] J.M.A.M. van Neerven, M. C. Veraar, and L.W. Weis. Stochastic integration in UMD Banach spaces. Ann. Probab., 35(4):1438-1478, 2007. MR2330977

[82] J.M.A.M. van Neerven, M.C. Veraar, and L.W. Weis. Stochastic integration in Banach spaces - a survey. In Stochastic Analysis: A Series of Lectures, volume 68 of Progress in Probability. Birkhäuser Verlag, 2015. MR3558130

[83] J.M.A.M. van Neerven and L. Weis. Stochastic integration of operatorvalued functions with respect to Banach space-valued Brownian motion. Potential Anal., 29(1):65-88, 2008. MR2421495

[84] A.A. Novikov. Discontinuous martingales. Teor. Verojatnost. $i$ Primemen., 20:13-28, 1975. MR0394861

[85] P. Nowak. On Jacod-Grigelionis characteristics for Hilbert space valued semimartingales. Stochastic Anal. Appl., 20(5):963-998, 2002. MR1938253

[86] P. Nowak. Integration with respect to Hilbert space-valued semimartingales via Jacod-Grigelionis characteristics. Stochastic Anal. Appl., 21(5):1141-1168, 2003. MR2000416

[87] B. Øksendal. Stochastic differential equations. Universitext. SpringerVerlag, Berlin, fifth edition, 1998. An introduction with applications. MR1619188

[88] M. Ondreját. Brownian representations of cylindrical local martingales, martingale problem and strong Markov property of weak solutions of SPDEs in Banach spaces. Czechoslovak Math. J., 55(130)(4):1003-1039, 2005. MR2184381

[89] A. Osekowski. On relaxing the assumption of differential subordination in some martingale inequalities. Electron. Commun. Probab., 16:9-21, 2011. MR2753300

[90] A. Osekowski. Sharp martingale and semimartingale inequalities, volume 72 of Instytut Matematyczny Polskiej Akademii Nauk. Monografie Matematyczne (New Series). Birkhäuser/Springer Basel AG, Basel, 2012. MR2964297

[91] A. Osekowski and I.S. Yaroslavtsev. The Hilbert transform and orthogonal martingales in Banach spaces. Int. Math. Res. Not. IMRN, https://doi. org/10.1093/imrn/rnz187, 2019.

[92] F. Papangelou. Integrability of expected increments of point processes and a related random change of scale. Trans. Amer. Math. Soc., 165:483-506, 1972. MR0314102

[93] S. Peszat and J. Zabczyk. Stochastic partial differential equations with Lévy noise. An evolution equation approach, volume 113 of Encyclopedia of Mathematics and its Applications. Cambridge University Press, Cambridge, 2007. MR2356959

[94] G. Pisier. Martingales in Banach spaces, volume 155. Cambridge University Press, 2016. MR3617459

[95] P.E. Protter. Stochastic integration and differential equations, volume 21 
of Stochastic Modelling and Applied Probability. Springer-Verlag, Berlin, 2005. Second edition. Version 2.1, Corrected third printing. MR2273672

[96] A.R. Pruss. Comparisons between tail probabilities of sums of independent symmetric random variables. Ann. Inst. H. Poincaré Probab. Statist., 33(5):651-671, 1997. MR1473569

[97] D. Revuz and M. Yor. Continuous martingales and Brownian motion, volume 293 of Grundlehren der Mathematischen Wissenschaften [Fundamental Principles of Mathematical Sciences]. Springer-Verlag, Berlin, third edition, 1999. MR1725357

[98] M. Riedle and O. van Gaans. Stochastic integration for Lévy processes with values in Banach spaces. Stochastic Process. Appl., 119(6):1952-1974, 2009. MR2519352

[99] H.P. Rosenthal. On the subspaces of $L^{p}(p>2)$ spanned by sequences of independent random variables. Israel J. Math., 8:273-303, 1970. MR0271721

[100] B.L. Rozovskiǔ. Stochastic evolution systems, volume 35 of Mathematics and its Applications (Soviet Series). Kluwer Academic Publishers Group, Dordrecht, 1990. Linear theory and applications to nonlinear filtering, Translated from the Russian by A. Yarkho. MR1135324

[101] J.L. Rubio de Francia. Martingale and integral transforms of Banach space valued functions. In Probability and Banach spaces (Zaragoza, 1985), volume 1221 of Lecture Notes in Math., pages 195-222. Springer, Berlin, 1986. MR0875011

[102] K.-i. Sato. Lévy processes and infinitely divisible distributions, volume 68 of Cambridge Studies in Advanced Mathematics. Cambridge University Press, Cambridge, 2013. Translated from the 1990 Japanese original, Revised edition of the 1999 English translation. MR3185174

[103] A.N. Shiryaev. Probability, volume 95 of Graduate Texts in Mathematics. Springer-Verlag, New York, second edition, 1996. Translated from the first (1980) Russian edition by R. P. Boas. MR1368405

[104] A.V. Skorohod. Limit theorems for stochastic processes. Teor. Veroyatnost. i Primenen., 1:289-319, 1956. MR0084897

[105] M.C. Veraar. Stochastic integration in Banach spaces and applications to parabolic evolution equations. PhD thesis, Delft University of Technology, 2006.

[106] M.C. Veraar. Continuous local martingales and stochastic integration in UMD Banach spaces. Stochastics, 79(6):601-618, 2007. MR2368370

[107] M.C. Veraar. Randomized UMD Banach spaces and decoupling inequalities for stochastic integrals. Proc. Amer. Math. Soc., 135(5):1477-1486, 2007. MR2276657

[108] M.C. Veraar and I.S. Yaroslavtsev. Cylindrical continuous martingales and stochastic integration in infinite dimensions. Electron. J. Probab., 21:Paper No. 59, 53, 2016. MR3563887

[109] M.C. Veraar and I.S. Yaroslavtsev. Pointwise properties of martingales with values in Banach function spaces. In High Dimensional Probability VIII, pages 321-340, Cham, 2019. Springer International Publishing. 
[110] F. Weisz. Martingale Hardy spaces with continuous time. In Probability theory and applications, volume 80 of Math. Appl., pages 47-75. Kluwer Acad. Publ., Dordrecht, 1992. MR1211899

[111] F. Weisz. Martingale Hardy spaces and their applications in Fourier analysis, volume 1568 of Lecture Notes in Mathematics. Springer-Verlag, Berlin, 1994. MR1320508

[112] W. Whitt. Stochastic-process limits. Springer Series in Operations Research. Springer-Verlag, New York, 2002. An introduction to stochasticprocess limits and their application to queues. MR1876437

[113] I.S. Yaroslavtsev. Weak $L^{1}$-estimates for weakly differentially subordinated martingales. In preparation.

[114] I.S. Yaroslavtsev. Brownian representations of cylindrical continuous local martingales. Infin. Dimens. Anal. Quantum Probab. Relat. Top., 21(2):1850013, 25, 2018. MR3819813

[115] I.S. Yaroslavtsev. Fourier multipliers and weak differential subordination of martingales in UMD Banach spaces. Studia Math., 243(3):269-301, 2018. MR3818326

[116] I.S. Yaroslavtsev. Martingale decompositions and weak differential subordination in UMD Banach spaces. Bernoulli, 25(3):1659-1689, 2019. MR3961226

[117] I.S. Yaroslavtsev. Martingales and stochastic calculus in Banach spaces. PhD thesis, Delft University of Technology, 2019.

[118] I.S. Yaroslavtsev. On the martingale decompositions of Gundy, Meyer, and Yoeurp in infinite dimensions. Ann. Inst. Henri Poincaré Probab. Stat., 55(4):1988-2018, 2019. MR4029146

[119] I.S. Yaroslavtsev. Burkholder-Davis-Gundy inequalities in UMD Banach spaces. Comm. Math. Phys., https://doi.org/10.1007/ s00220-020-03845-7, 2020.

[120] Ch. Yoeurp. Décompositions des martingales locales et formules exponentielles. In Séminaire de Probabilités, X (Seconde partie: Théorie des intégrales stochastiques, Univ. Strasbourg, Strasbourg, année universitaire 1974/1975), pages 432-480. Lecture Notes in Math., Vol. 511. Springer, Berlin, 1976. MR0451395

[121] J. Zhu, Z. Brzeźniak, and W. Liu. Maximal Inequalities and Exponential Estimates for Stochastic Convolutions Driven by Lévy-type Processes in Banach Spaces with Application to Stochastic Quasi-Geostrophic Equations. SIAM J. Math. Anal., 51(3):2121-2167, 2019. MR3953459

[122] J. Zinn. Comparison of martingale difference sequences. In Probability in Banach spaces, V (Medford, Mass., 1984), volume 1153 of Lecture Notes in Math., pages 453-457. Springer, Berlin, 1985. MR0821997 